\title{
Motor learning in neurological rehabilitation : practising skills with movement imagery
}

Citation for published version (APA):

Braun, S. M. (2010). Motor learning in neurological rehabilitation : practising skills with movement imagery. [Doctoral Thesis, Maastricht University]. Datawyse / Universitaire Pers Maastricht. https://doi.org/10.26481/dis.20100623sb

Document status and date:

Published: 01/01/2010

DOI:

$10.26481 /$ dis.20100623sb

Document Version:

Publisher's PDF, also known as Version of record

\section{Please check the document version of this publication:}

- A submitted manuscript is the version of the article upon submission and before peer-review. There can be important differences between the submitted version and the official published version of record. People interested in the research are advised to contact the author for the final version of the publication, or visit the DOI to the publisher's website.

- The final author version and the galley proof are versions of the publication after peer review.

- The final published version features the final layout of the paper including the volume, issue and page numbers.

Link to publication

\footnotetext{
General rights rights.

- You may freely distribute the URL identifying the publication in the public portal. please follow below link for the End User Agreement:

www.umlib.nl/taverne-license

Take down policy

If you believe that this document breaches copyright please contact us at:

repository@maastrichtuniversity.nl

providing details and we will investigate your claim.
}

Copyright and moral rights for the publications made accessible in the public portal are retained by the authors and/or other copyright owners and it is a condition of accessing publications that users recognise and abide by the legal requirements associated with these

- Users may download and print one copy of any publication from the public portal for the purpose of private study or research.

- You may not further distribute the material or use it for any profit-making activity or commercial gain

If the publication is distributed under the terms of Article $25 \mathrm{fa}$ of the Dutch Copyright Act, indicated by the "Taverne" license above, 
Susy M. Braun

\section{Motor learning in neurological rehabilitation: practising skills with movement imagery}


ISBN 9789052789590

Cover and illustrations: Anneliese Braun-Specht

Theme Cover illustration: Engelen kunnen vliegen omdat zij zo luchtig over zichzelf denken. (G.K. Chesterton)

Graphic Design: Aline van Hoof, Landgraaf

Printing: Universitaire Pers Maastricht

HOGESCHOOL
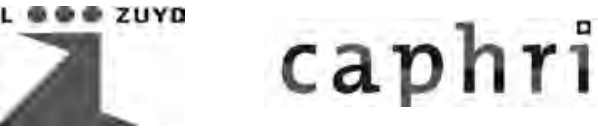

Copyright (C) S. Braun, Maastricht 2010

All rights reserved. No parts of this thesis may be reproduced or transmitted in any form or by any means, electronic or mechanical, including photocopying, recording or any information storage or retrieval system, without permission in writing from the author, or when appropriate, from the publisher of the publication.

Note

As some chapters have been published in British and others in American journals, the used language style is not uniform. Therefore, certain words can sometimes be spelled in UK and sometimes in US-English. Journals also have their own author guidelines regarding headings, structure of the manuscript, word count and other preferences in lay-out. Chapters 2 through 9 therefore also differ with regard to these formatting aspects. 


\section{Motor learning in neurological rehabilitation: practising skills with movement imagery}

Dissertation

to obtain the degree of Doctor

at the Maastricht University

on the authority of the Rector Magnificus

Prof. Dr. G.P.M.F. Mols

in accordance with the decision of the Board of Deans

to be defended in public

on Wednesday, June 23rd 2010, at 16.00 hours

by

Susy Michelle Braun 


\section{Promotores}

Prof. Derick T. Wade

Prof. Dr. Jos M.G.A. Schols

\section{Copromotor}

Dr. Anna J.H.M. Beurskens

\section{Assessment committee}

Prof. Dr. Jan P.H. Hamers (Chairman)

Prof. Dr. Rob A. de Bie

Prof. Dr. J. André Knottnerus

Prof. Dr. Gert Kwakkel (VU, University Medical Centre Amsterdam)

Dr. Nikhil Sharma (NINDS, NIH, United States of America)

The study presented in this thesis was embedded in the research centre of autonomy and participation of the chronic ill, the centre of expertise in life sciences and the department of health and technique of the Zuyd University of applied sciences and the research centre CAPHRI of the Maastricht University both in the Netherlands.

Parts of the research within this dissertation were financially supported by the Zuyd University of applied sciences (research centre for autonomy and participation of the chronic ill, centre of expertise in life sciences, department for health and technique and the board of executives), the Mobiliteitsfonds of the HBO-raad, ZonMW, health care insurance companies zorgverzekeraar VGZ and NutsOhra.

The publication of this thesis was financially supported by the research centre for autonomy and participation of the chronic ill, the department of health and technique, and the board of executives of the Zuyd University of applied sciences, Braunspecht b.v. and pcdata holding. 
Logic will get you from A to B, imagination will take you everywhere.

(Albert Einstein)

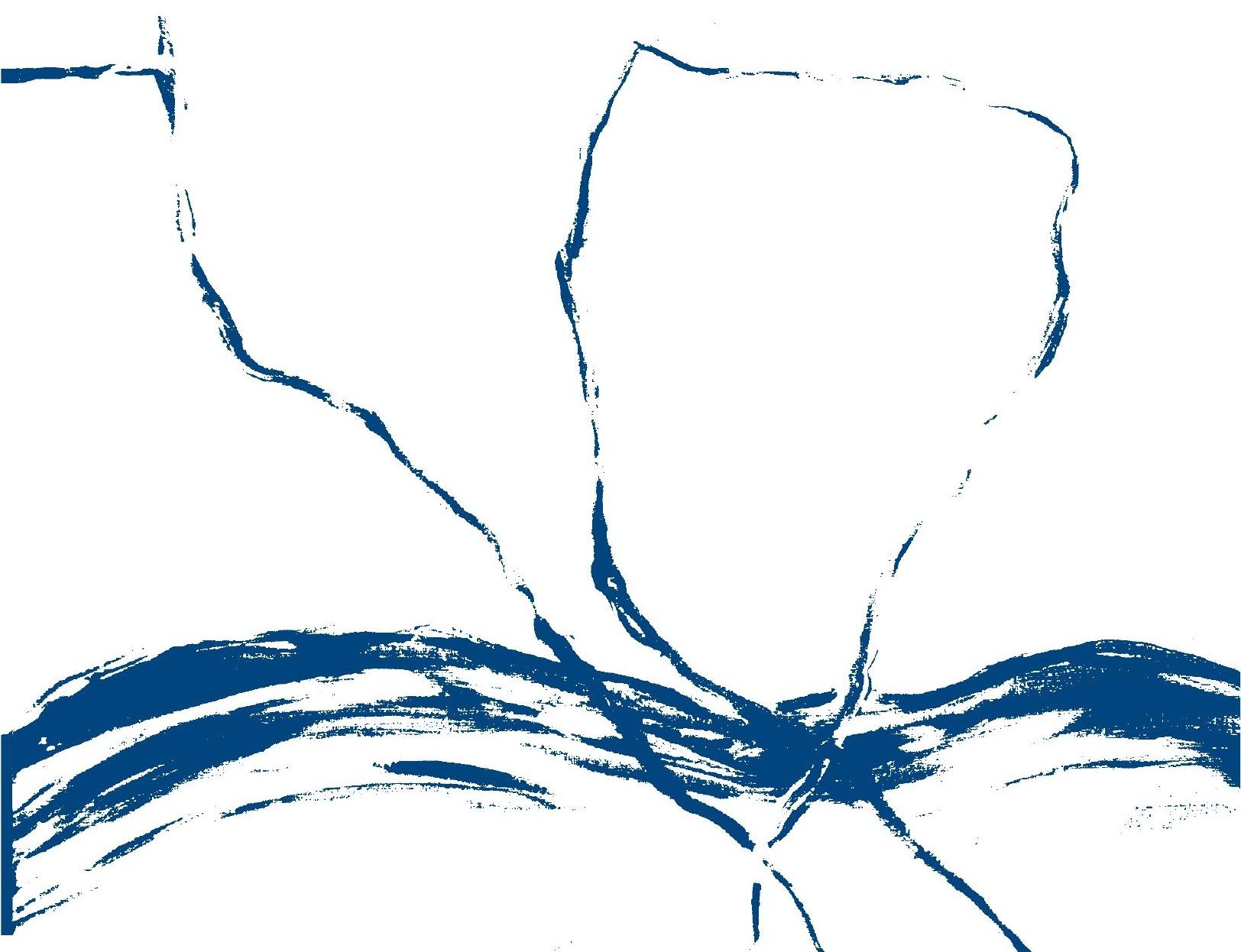




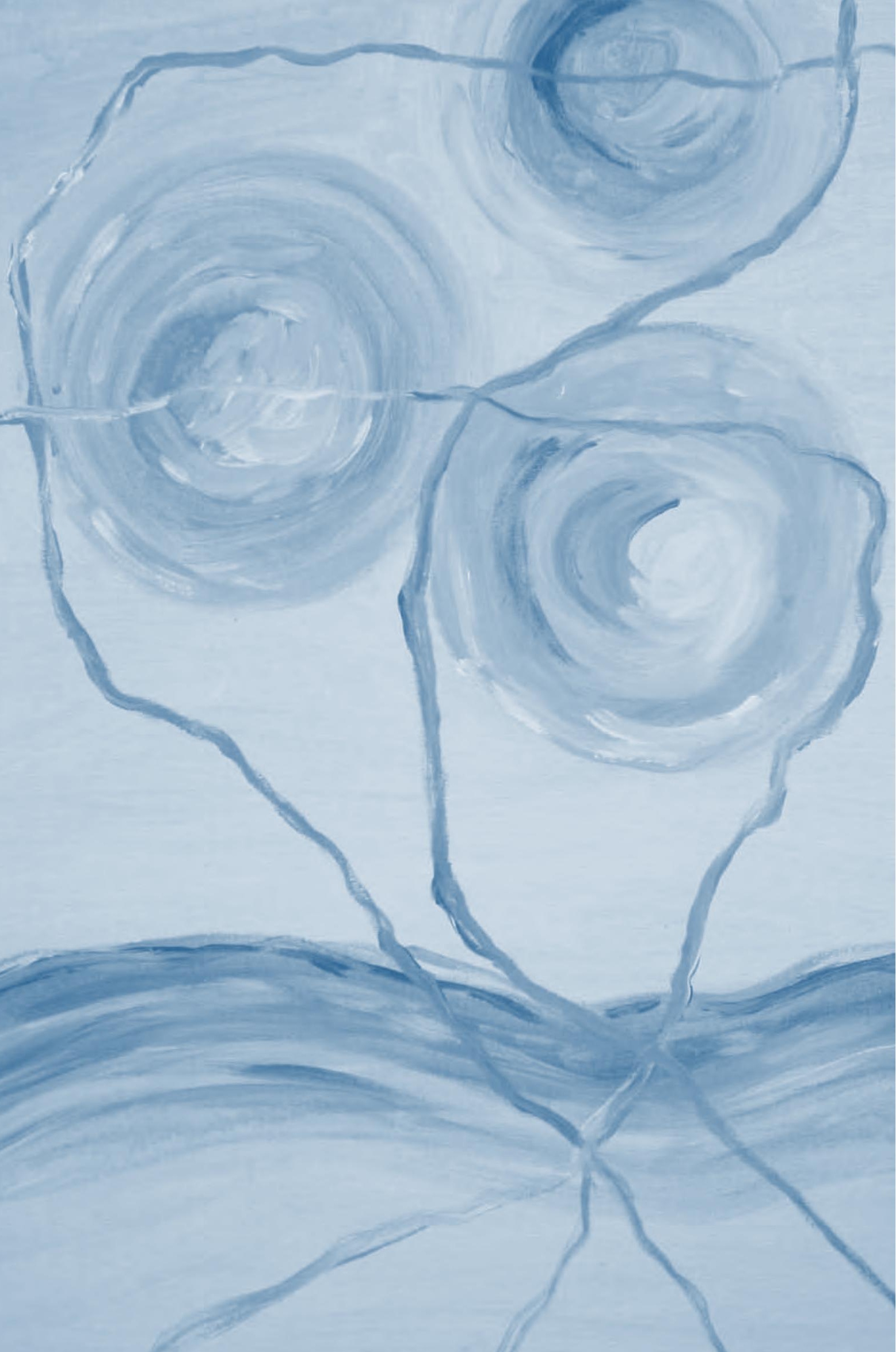




\section{Content}

Chapter one Introduction

Chapter two The effects of mental practice in stroke rehabilitation:

a systematic review

Chapter three Is it possible to use the Structural Dimension Analysis

of Motor memory (SDA-M) to investigate representations

of motor actions in stroke patients?

Chapter four Using mental practice in stroke rehabilitation:

a framework

Chapter five Effects of mental practice embedded in daily therapy compared to therapy as usual in adult stroke patients in Dutch nursing homes: design of a randomised controlled trial

Chapter six Feasibility of a mental practice intervention in stroke patients in Dutch nursing homes: a process evaluation

Chapter seven Effects of mental practice in stroke rehabilitation in

Dutch nursing homes: results from a randomised controlled trial

Chapter eight Using mental practice in patients with Parkinson's disease: results from a randomised controlled trial

Chapter nine The experience of movement imagery therapy by patients with stroke or Parkinson's disease 165

Chapter ten General discussion

Summary

Samenvatting (Dutch summary)

Appendix

Log example

About the author

Publications 


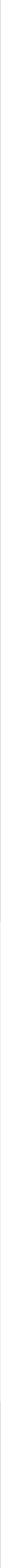




\section{Introduction}

\section{Motor learning in neurological rehabilitation: practising skills with movement imagery}

This $\mathrm{PhD}$-thesis used following background theory of motor learning as a starting point:

'All skilled movements are first undertaken using conscious, cognitively-driven control. Over time, with practice, they are learned. Learning implies information processing and the laying down somewhere of new neural pathways and connections that can be activated when the specific movement pattern is required. These pathways are probably held in neural networks as a specific pattern of interconnections. Each set is a motor action plan that defines and executes a more-or-less specific pattern of specific muscle actions. When the brain is damaged or disrupted by disease the networks may be disrupted, and consequently the motor action plans disturbed or even lost. Recovery from (improvement after) loss of neurological tissue will involve, restoring old or developing new motor action plans. In this project, we researched the potential use of movement imagery and mental practice for exactly that purpose, learning daily activities, in patients after stroke and patients with Parkinson's disease'.

While it is reasonably established that the overall process of neurological rehabilitation is effective, there is little evidence to support many specific rehabilitation therapeutic techniques. Currently it seems that task orientated practice (i.e. practising an activity of relevance) is the most effective single therapeutic technique $(1,2)$. This is similar to the situation in sport where practice is the bedrock of improving skills. Indeed improving any skilled motor activity seems to depend upon continuing practice (3-6). We therefore used the knowledge from sport science as a starting point, to develop and evaluate a mental practice intervention in neurorehabilitation.

\section{Imagery in sports}

Many books on psychological skills in sports start with a quotation from a famous athlete, telling us how mental strength contributed to their success. Elite athletes, like Inge de Bruijn (Dutch swimmer and Olympic Champion), Pat Cash (Australian tennis player and winner of Wimbledon), Erben Wennemars (Dutch ice skater and World Champion) talk about the power they believe thought has over actual performance. Trainers and athletes consider psychological skills, like psyching up, focusing, goal setting and mental practice as valuable additions to physical training (7). Moreover, mental training is often an accepted and substantial part of professional training schedules. 
One of the skills used in sport to improve motor skills is movement imagery. It is defined as imagining oneself undertaking the skilled movement without actually doing the movement, generating from thought as much kinesthetic and visual experience as possible. The subject may image the movement in the third (as if looking from a distance at oneself) or the first (as if looking through their own eyes) person's view (8). When these images are used regularly as a training method to enhance training load, it is called mental practice (9).

During mental practice, an internal representation of the movement is activated and the execution of the movement repeatedly mentally simulated, without physical activity, within a chosen context. It is used for the improvement or stabilization of a given movement (10).

Thus imagery has been a constant area of interest in sports science for a long time. Imagery as a research topic however, has known heights and lows. At the beginning of the 20th century, research was restricted to a few research groups, resulting in irregular publications. From the 1970ies on, these wave-like peaks have become steadier with research reports in sports reaching heights every four years, just after the Olympic Games (11).

\section{Explaining imagery}

A host of different theories has been used to explain the effects of mental practice. Important hypotheses include: symbolic learning theory, the attention/arousal-set theory, bio-informational theory and the ecological/action- based theory $(9,15-19)$.

These models of explanation differ on whether body-peripheral (neuromuscular) processes or central mechanisms (such as representations or programs) are the origin of these effects (9, 12, 13). Jacobson (1932) for example postulated that during mental practice subliminal impulses reached the muscles and caused a non visible contraction there, building up tonus and strengthening the coordinative pathways, but not showing any overt movement (so called 'efferent leakage') (14). Later this hypothesis was confirmed by other researchers using EMG-measurements (12).

Schack and co-workers are proponents of the theory that central mechanisms are causing the effects. The paradigm is that goal-directed movements are organised and stored in memory as 'events' by way of representation of their anticipated perceptual effects. They proposed the existence of 'basic action concepts' or 'motor representations' which 'can be considered as elementary components or transitional states of complex movement with specific functional and perceptual features'. Basic action concepts can be described verbally or through visual images. They are stored in long term memory and are moved into the working memory being placed in the correct order and adapted to the biomechanical demands in a specific environment before being activated to perform the action (13). 
Unfortunately these theories individually only explain parts of the imagery puzzle (9). A plausible reason for not being able to explain imagery and the underlying working mechanisms as a whole or total concept, is the methodological problems in imagery research undertaken so far. Too many different interventions, tasks, populations and measures were and are all captured in this kind of research (20-22).

One particular difficulty is that several different imagery concepts are subsumed within the phrase 'mental imagery' and at the same time several different phrases may be used to describe the same intervention - 'mental practice', 'motor imagery', 'mental rehearsal' and so on. Thus when imagery effects are being assessed, many interventions and terms, all somehow related to imagery and/or mental practice, are used. Consequently every article must be read in detail to understand what the authors have done, but unfortunately there is often either no description, or only a vague, imprecise and minimal description.

This problem was recognized in the 1990ies by different authors like Feltz and Landers (22), Budney and co-workers (20) and Driskel and co-workers (21) and put into words in article titles like 'Imagery and motor performance, what do we really know?'(20)

Eventually a theoretical framework was developed by Martin and co-workers in 1999 to cope with this variation in imagery research (23). The applied model differentiated between different imagery types and consequently outcomes, aiming at gearing research to one another.

\section{Brain imaging research}

These conceptual and methodological issues have encouraged marked criticism of movement imagery and mental practice research over most of the last 100 years. This seemed to change in the 1990's when the technical possibilities of (functional) magnetic resonance imaging, positron emission tomography and (quantitative) electro-encephalography increased fundamental research on brain activity.

The article, 'Reopening the mental imagery debate: lessons from functional anatomy', by Mellit and Petit (1998) reviews what happened at the end of the 20th century (24). The results from brain imaging research showed that during both imagery and actual execution of a skill almost exactly the same brain areas were active $(23,25-27)$. This phenomenon allowed the development of computer-brain-interfaces (28).

Suddenly an explanation for the (putative) effects of imagery was found, a foundation that even the biggest imagery critics could not deny. These research findings were a huge impulse for sports. It was perhaps also the reason why suddenly imagery was considered as an additional technique in rehabilitation $(29,30)$. 
Imagery in rehabilitation

An important paper encouraging imagery research in rehabilitation was written by Jackson and co-workers, 'Potential role of mental practice using motor imagery in neurologic rehabilitation', published in 2001 (29). Few research groups around Page, Liu and Malouin were the first to pick up this research area on a clinical level, testing the use of imagery in therapy of neurological patients. Approximately 10 effect studies in stroke and various ability studies were published within the first five years (31-42).

Here is where our research project started five years ago.

Our main aims were:

- $\quad$ to review the existing evidence;

- to try a technique for exploring motor plans;

- to develop an imagery intervention for modifying motor plans;

- $\quad$ and to evaluate the whole package (identifying the disrupted plan and having a focused method for restoring it) in two neurological populations: stroke and Parkinson's disease.

The figure below gives an overview of the development of this project with references to the separate chapters. In order for the reader to follow our thoughts, we chose to describe the project in a chronological order.

\section{5}

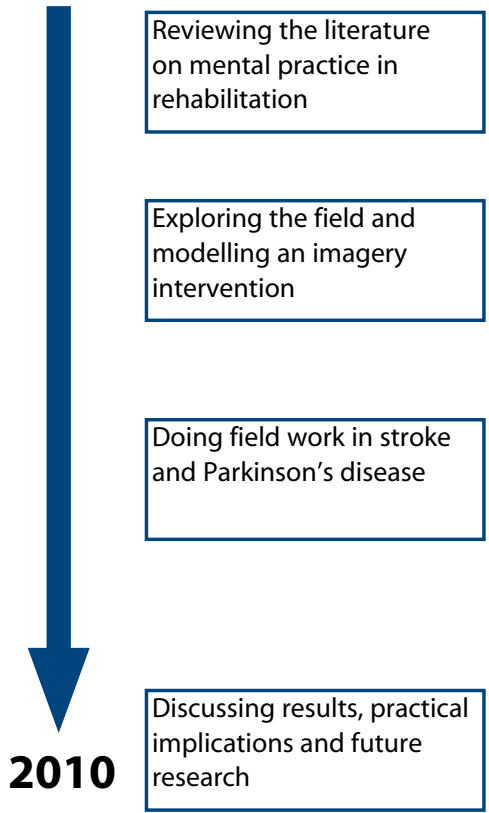

$\longrightarrow$ Chapter two: evidence; systematic review (p 15)

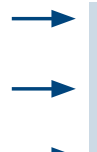

Chapter three: representations of motor actions; SDA-M (p 39)

$\longrightarrow$ Chapter five: design of the stroke trial ( $p$ 77)

Chapter four: intervening with mental practice; framework ( $\mathrm{p}$ 57)

$\longrightarrow$ Chapter six: process evaluation of the stroke trial (p 103)

$\longrightarrow$ Chapter seven: results from the stroke trial (p 123)

$\longrightarrow$ Chapter eight: results from the Parkinson's trial (p 143)

$\longrightarrow$ Chapter nine: perceptions of patients on imagery (p 165) 
Before we started our clinical studies, we wanted to know how much evidence there was for imagery in stroke rehabilitation. In chapter two, our systematic review, 'The effects of mental practice in stroke rehabilitation: a systematic review', is described (10). We encountered the same problems as in sports literature and sports research: many different interventions, tasks, populations and measures were all captured under the concept of 'imagery' and this made comparison and meta-analysis of studies impossible. This was also the main conclusion in other later published reviews and critical discussions (43-45).

Studies involving brain imaging techniques in patients with neurological disease have taken place over the last few years, and the results suggests that imagery ability depends upon the neurological disease and more specifically the localization of the disease (46-48). In at least some neurological patients however, executed and imagined movements seem to share a common neural substrate (49-54).

We were looking for an easy and low cost way to measure what people potentially could be thinking during the imagery of movement and more importantly, if they were able to image movements in the correct sequence. Through the work of the psychology department of the Sports University in Cologne we discovered the SDA-M. In sports science, a computer program to assess the cognitive structure of motor skills, the Structural Dimensional Analysis of Motor memory (SDA-M), was developed to distinguish differences in motor representations between novices and experts (55). It has been tested in many sports and significant differences in 'thinking about movements' have been found in gymnasts, sky divers, tennis players, surfers, etc. (56). The SDA-M is used to determine the basic architecture of specific goal-directed movements, for example to identify weak spots in the sequence of events that should lead to a certain motor performance, such as when a volleyball player is having trouble with hitting a smash.

Furthermore, it is assumed that both movement imagery and movement execution are based on the same representation. This has lead to the hypothesis that mental practice can contribute to the activation of neural loops and patterns of movements of which the brain has a sort of motor print (or map). Mental practice could therefore be used in addition to physical rehabilitation in patients with neurological disorders, especially in the early stage of recovery, the stage where reorganizing brain networks and patterns is of great importance.

The SDA-M seemed worthwhile to investigate in rehabilitation. In order to assess whether or not patients are imaging the skills they wanted to improve correctly, and perhaps to adjust this representation before practising mentally, we explored if the SDA-M could be used in rehabilitation for everyday tasks (chapter three: 'Is it possible to use the Structural Dimension Analysis of Motor memory (SDA-M) to investigate representations of motor actions in stroke patients?' (57)). 
Imagery is a natural part of thinking and many people use it unconsciously to recall information stored in the brain but not directly accessible ('where did I leave my keys?' (9)). It was therefore not surprising, that many therapists reported they were (unsystematically) already using imagery when we first started talking to the treating therapists about our ideas.

It soon became clear that different therapists had different understanding of the concept of imagery, depending on their expertise, working experience and preferences. The same accounted for the researchers: in the reported trials, there also was no consensus among the researcher on how to teach, improve and monitor mental practice. At a very early stage, we decided to do justice to the way professionals work in routine care while nonetheless standardizing practice by developing an imagery treatment framework instead of a fixed instruction book (58). This thought is in line with the independent way of decision making and working within the professional multidisciplinary team in the Netherlands $(3,6,58)$. Mental practice is tailored to the abilities and preferences of the patient.

The development of a mental practice intervention was also an attempt to define mental practice and unify the mental practice intervention and describe how mental practice could be taught in rehabilitation. In chapter four the framework is described ('Using mental practice in stroke rehabilitation: a framework' (59)). It also became clear, that mental practice is a complex intervention with many challenges, especially for patients with neurological disorders.

We explored our preliminary work by testing it as a feasibility study in a Dutch nursing home. We didn't need to adjust the mental practice framework, but saw some improvement points in the assisting materials, organisation structures and communication routes (which are always specific to the setting or location). As a final testing step, a couple of single cases in outpatients of the Oxford centre for enablement in Oxford, the UK, was performed. The treatment seemed feasible and first results promising (60).

Because the effects of mental practice in stroke rehabilitation were still uncertain, we decided to do a clinical study on effects of imagery in stroke patients, in the sub acute phase of recovery, in nursing homes. The underlying thoughts and decisions were reported in a design article ('Effects of mental practice embedded in daily therapy compared to therapy as usual in adult stroke patients in Dutch nursing homes: design of a randomised controlled trial' (61) chapter five). We specifically chose this sub group within the stroke population for three reasons: (1) They are a huge part of the entire population and if imagery were effective in these patients a large group would benefit from it; (2) We reasoned that patients in the sub acute phase of recovery were most likely to benefit most from mental practice as plasticity of the brain can be used to its full potential (62, 
63) during the first three months of main recovery; and (3), we hypothesized that patients might recall the sensations of normal motor control and movement better shortly after their stroke than months later.

The randomised controlled trial was evaluated in a detailed process evaluation. The aims of this study were to assess 1) whether the conditional and optional part of the mental practice intervention were performed according to the framework and 2) therapists' and participants' experiences with the intervention. Results from this evaluation are reported in chapter six: 'Feasibility of a mental practice intervention in stroke patients in Dutch nursing homes: a process evaluation' (64).

In most imagery research in rehabilitation or therapy the emphasis is on the effects on increasing the level of activity (i.e. the effects on reducing disability). The goal of our study was the same. The results from the stroke trial are reported in chapter seven 'Effects of mental practice in stroke rehabilitation in Dutch nursing homes: results from a randomised controlled trial' (65).

In 2007, a more general review by Dickstein and co-workers on the effects of imagery in physiotherapy was published (44). They described possible effects of imagery not only in stroke patients, but also in chronic pain patients, patients with spinal cord injury and patients with Parkinson's disease. By this time, we had also become curious about possible effects of mental practice in patients with Parkinson's disease (66).

The contrast between the pathologies (stroke versus Parkinson's disease) was one important reason. Another was the possible comparison between the use of mental practice by several therapists in a nursing home on inpatients and by a community-based physiotherapist seen people as day patients. A pilot study with patients in the community was carried out. Four fundamental studies on the ability to perform imagery and two clinical studies were found to support this side step in our research $(46-48,66,67)$. Implementation possibilities of the imagery framework and first results are reported in chapter eight 'Using mental practice in patients with Parkinson's disease: results from a randomised controlled trial' (68).

The beliefs, preferences and expectations of the clients and the therapists are important in rehabilitation. We found one study by Sharma and co-workers in which stroke patients were interviewed on this subject (72).

Recently more qualitative research is performed in sports to gain better insight into what athletes are actually doing during imagery and is called 'the second wave of imagery research' (69). The 'four w's', originally described by Monroe and co-workers (70), was continued in research by 
Driediger and co-workers. Interviews with injured athletes revealed interesting information on why athletes use imagery, what they do during mental practice (and how), when they practice and where. The answer to the last question was a bit disturbing, as many athletes reported to practice mentally while driving! (71).

We decided to perform qualitative research in order to investigate this aspect further. Patients after stroke or with Parkinson's disease were interviewed individually or in a group. Results are reported in chapter nine 'The experience of movement imagery therapy by patients with stroke or Parkinson's disease' (73).

In chapter ten we discuss the results of this PhD-project ('General discussion'). The guidance from the Medical Research Council for developing and evaluating complex interventions is used to compare our findings with results from other research. Where do we stand at this point? And where should future imagery research focus on?

\section{References}

1. Ma HI, Trombly CA, Robinson-Podolski C. The effect of context on skill acquisition and transfer. Am J Occup Ther 1999;53:138-44.

2. Trombly CA, Wu CY. Effect of rehabilitation tasks on organization of movement after stroke. Am J Occup Ther 1999;53:333-44.

3. Commissie CVA-revalidatie. Revalidatie na een beroerte, richtlijnen en aanbevelingen voor zorgverleners. Den Haag: Nederlandse Hartstichting; 2001

4. Cup E, Stultjens EM: Ergotherapie richtlijn beroerte. Utrecht: Nederlandse Vereniging Ergotherapie; 2005.

5. Keus SHJ, Hendriks HJM, Bloem BR, Bredero-Cohen AB, de Goede CJT, van Haaren M, et al. KNGF-Richtlijn 'Parkinson'. Ned Tijdschr Fysiother 2004;114(Suppl):1-86.

6. van Peppen RP, Kwakkel G, Harmeling-van der Wel BC, et al.: KNGF-richtlijn beroerte. Ned Tijdschr Fysiother 2004;114(Suppl):1-77.

7. Taylor J, Wilson G. Applying sport psychology. Four perspectives. Champaign, IL: Human Kinetics; 2005.

8. Hale BD. Imagery perspectives and learning in sports performance. In: Sheikh AA, Korn ER, editors. Imagery in sports and physical performance. Farmingdale (NY): Baywood;1994. p 75-96.

9. Boschker M. Action-Based Imagery. On the nature of mentally imagined motor actions [Dissertation]. Amsterdam, The Netherlands:Vrije Universiteit Amsterdam Ipskamp PrintPartners; 2001. 
10. Braun SM, Beurskens AJ, Borm PJ, Schack T, Wade DT. The effects of mental practice in stroke rehabilitation: a systematic review. Arch Phys Med Rehabil 2006;87:842-52.

11. Schuster C, Braun SM. Mentale Bewegungsvorstellungen - ein (un)bekannter Therapieansatz beim Wiedererlernen von Bewegungsabläufen nach Störungen des Zentralen Nervensystems. Workshop at the Schweizer National Kongress, St. Gallen, 13-14 Juni 2008.

12. Bakker FC, Boschker MS, Chung T: Changes in muscular activity while imagining weight lifting using stimulus or response proportions. J Sport Exerc Psychol 1996;18:313-24.

13. Schack T. The cognitive architecture of complex movements. International J Sport Exerc Psychol 2004;2(4):403-38.

14. Jacobson E. Electrophysiology of mental activities. American Journal of Physiology. 1932;44: $677-49$.

15. Drobes DJ, Lang PJ. Bioinformational theory and behaviour therapy. In: O'Donohue W, Krasner L, editors. Theories of behaviour therapy: Exploring behaviour change. Washington DC: American Psychological Association; 1995. p 229-57.

16. Gibson JJ. The ecological approach to visual perception. Boston, MA: Houghton Mifflin; 1979.

17. Lang PJ. Cognition and emotion: Concept and action. In: Izard CE, Kagan, J, Zajonc RB, editors. Emotions, cognition and behaviour. New York: Cambridge University Press; 1984. p 192-226.

18. Pavio A. Images in Mind: The evolution of a theory. Hertfordshire, GB: Harvester, Wheatsheaf, Hemel \& Hempstead; 1991.

19. Sackett RS. The relationship between amout of symbolic rehearsal and retention of a maze habit. Journal of General Psychology 1935;13:113-28.

20. Budney AJ, Murphy SM, Woolfolk RL. Imagery and Motor performance: What do we really know? In: Sheikh AA, Korn ER, editors. Imagery in sports and physical performance. Farmingdale (NY): Baywood; 1994. p 97-120.

21. Driskell JE, Copper C, Moran A. Does mental practice enhance performance? Journal of applied psychology 1994;79:481-92.

22. Feltz, DL, Landers D. A revised meta-analysis of the mental practice literature on motor skill learning and performance. In: Druckman D, Swets J, editors. Enhancing human performance: issues, theories and techniques. Washington DC: Nat Acad Sc; 1988. p 1-65.

23. Martin K, Moritz S, Hall C. Imagery Use in Sports: A Literature Review and Applied Model. The Sports Psychologist 1999;13:245-68.

24. Mellit E, Petit L. Reopening the mental imagery debate: Lessons from functional anatomy. Neuroimage 1998;8:129-39.

25. Decety J. The neurophysiological basis of motor imagery. Behav Brain Res 1996;77:45-52.

26. Jeannerod M. Mental imagery in the motor cortex. Neuropsychologia 1995;33:1419-32. 
27. Nair DG, Purcott KL, Fuchs A, Steinberg F, Kelso JA. Cortical and cerebellar activity of the human brain during imagined and executed unimanual and bimanual action sequences: a functional MRI study. Brain Res Cogn Brain Res 2003;15:250-60.

28. Pfurtscheller G, Neuper C, Andrew C, Edlinger G. Foot and hand area mu rhythms. Int J Psychophysiol 1997;26:121-35.

29. Jackson PL, Lafleur MF, Malouin F, Richards C, Doyon J. Potential role of mental practice using motor imagery in neurologic rehabilitation. Arch Phys Med Rehabil 2001;82:1133-41.

30. van Leeuwen R, Inglis, J. Mental practice and imagery: a potential role in stroke rehabilitation. Physical Therapy Reviews 1998;3:47-52.

31. Dickstein R, Dunsky A, Marcovitz E. Motor imagery for gait rehabilitation in post-stroke hemiparesis. Phys Ther 2004;84:1167-77.

32. Dijkerman HC, Ietswaart M, Johnston M, MacWalter RS. Does motor imagery training improve hand function in chronic stroke patients? A pilot study. Clin Rehabil 2004;18: $538-49$.

33. Dunsky A, Dickstein R, Ariav C, Deutsch J, Marcovitz E. Motor imagery practice in gait rehabilitation of chronic post-stroke hemiparesis: four case studies. Int J Rehabil Res 2006;29:351-6.

34. Liu KP, Chan CC, Lee TM, Hui-Chan CW. Mental imagery for relearning of people after brain injury. Brain Inj 2004;18:1163-72.

35. Liu KP, Chan CC, Lee TM, Hui-Chan CW. Mental imagery for promoting relearning for people after stroke: a randomized controlled trial. Arch Phys Med Rehabil 2004;85:1403-8.

36. Malouin F, Richards CL, Desrosiers J, Doyon J. Bilateral slowing of mentally simulated actions after stroke. Neuroreport 2004;15:1349-53.

37. Malouin F, Richards CL, Doyon J, Desrosiers J, Belleville S. Training mobility tasks after stroke with combined mental and physical practice: a feasibility study. Neurorehabil Neural Repair 2004;18:66-75.

38. Malouin F, Richards CL, Jackson PL, Dumas F, Doyon J. Brain activations during motor imagery of locomotor-related tasks: a PET study. Hum Brain Mapp 2003;19:47-62.

39. Page SJ. Mental practice: a promising restorative technique in stroke rehabilitation. Top Stroke Rehabil 2001;8:54-63.

40. Page SJ, Levine P, Leonard AC. Effects of mental practice on affected limb use and function in chronic stroke. Arch Phys Med Rehabil 2000;86:399-402.

41. Page SJ, Levine P, Sisto S, Johnston MV. A randomized efficacy and feasibility study of imagery in acute stroke. Clin Rehabil 2001;15:233-40.

42. Stevens JA, Stoykov ME. Using motor imagery in the rehabilitation of hemiparesis. Arch Phys Med Rehabil 2003;84:1090-2. 
43. de Vries S, Mulder T. Motor imagery and stroke rehabilitation: a critical discussion. J Rehabil Med 2007;39:5-13.

44. Dickstein R, Deutsch JE. Motor imagery in physical therapist practice. Phys Ther 2007;87: 942-53.

45. Sharma N, Pomeroy VM, Baron JC. Motor imagery: a backdoor to the motor system after stroke? Stroke 2006;37:1941-52.

46. Cunnington R, Egan GF, O'Sullivan JD, Hughes AJ, Bradshaw JL, Colebatch JG. Motor imagery in Parkinson's disease: a PET study. Mov Disord 2001;16:849-57.

47. Frak V, Cohen $\mathrm{H}$, Pourcher E. A dissociation between real and simulated movements in Parkinson's disease. Neuroreport 2004;15:1489-92.

48. Helmich RC, de Lange FP, Bloem BR, Toni I. Cerebral compensation during motor imagery in Parkinson's disease. Neuropsychologia 2007;45:2201-15.

49. Carey L, Seitz RJ. Functional neuroimaging in stroke recovery and neurorehabilitation: conceptual issues and perspectives. International journal of stroke 2007;2:245-64.

50. Fogassi L, Ferrari PF, Gesierich B, Rozzi S, Chersi F, Rizzolatti G. Parietal lobe: from action organization to intention understanding. Science 2005;308:662-7.

51. Johansen-Berg H. Functional imaging of stroke recovery: what have we learnt and where do we go from here? International journal of stroke 2007;2:7-16.

52. Johnson SH. Imagining the impossible: intact motor representations in hemiplegics. Neuroreport 2000;11:729-32.

53. Johnson SH, Sprehn G, Saykin AJ. Intact motor imagery in chronic upper limb hemiplegics: evidence for activity-independent action representations. J Cogn Neurosci 2002;14:841-52.

54. Samuel M, Ceballos-Baumann AO, Boecker H, Brooks DJ. Motor imagery in normal subjects and Parkinson's disease patients: an H215O PET study. Neuroreport 2001;12:821-8.

55. Schack T. The cognitive architecture of movement. J Sport Exerc Psychol 2004;2(4).

56. Schack T, Mechsner F. Representation of motor skills in human long-term memory. Neurosci Lett 2006;391:77-81.

57. Braun SM, Schack T, Marcellis RG, Oti KC, Schols JM, Wade DT, Beurskens AJ. Representations of motor actions in stroke patients and healthy elderly. Clin Rehabil 2007;21:822-32.

58. Wade DT. Personal context as a focus for rehabilitation. Clin Rehabil 2000;14:115-8.

59. Braun SM, Kleynen M, Schols JM, Schack T, Beurskens AJ, Wade DT. Using mental practice in stroke rehabilitation; a framework. Clin Rehabil 2008;22:579-591.

60. Kleynen M, Schoenmakers M, Braun SM. Stel je voor, het werkt. Mentale training bij een chronische CVA patiënt: een case report. Ned Tijdschr Fysiother 2009;119:48-54. 
61. Braun SM, Beurskens AJ, van Kroonenburgh SM, Demarteau J, Schols JM, Wade DT. Effects of mental practice embedded in daily therapy compared to therapy as usual in adult stroke patients in Dutch nursing homes: design of a randomised controlled trial. BMC Neurol 2007;7:34.

62. Burke SN, Barnes CA. Neural plasticity in the ageing brain. Nat Rev Neurosci 2006;7:30-40.

63. Kleim JA, Jones TA. Principles of experience-dependent neural plasticity: implications for rehabilitation after brain damage. J Speech Lang Hear Res 2008;51:225-39.

64. Braun SM, van Haastregt JC, Beurskens AJ, Wade DT, Gielen X, Schols JM. Feasibility of a mental practice intervention in stroke patient in nursing homes; a process evaluation. (Submitted 2009)

65. Braun SM, Beurskens AJ, Kleynen M, Oudelaar B, Schols JM, Wade DT. Effects of mental practice in stroke rehabilitation in Dutch nursing homes: results from a randomised controlled trial. (Submitted 2010)

66. Tamir R, Dickstein R, Huberman M. Integration of motor imagery and physical practice in group treatment applied to subjects with Parkinson's disease. Neurorehabil Neural Repair 2007;21:68-75.

67. Yaguez L, Canavan AG, Lange HW, Homberg V. Motor learning by imagery is differentially affected in Parkinson's and Huntington's diseases. Behav Brain Res 1999;102:115-27.

68. Braun SM, Beurskens AJ, Kleynen M, Schols JM, Wade DT. Using mental practice in patients with Parkinson's disease; results from a randomised controlled trial. (Submitted 2010)

69. MacIntyre TE, Moran AP. A qualitative investigation of imagery use and meta-imagery processes among elite canoe-slalom competitors. Journal of imagery research in sport and physical activity 2007;2:1-19.

70. Munroe K, Giacobbi P, Hall C, Weinberg R. The four w's of imagery use: Where, when, why and what. The Sport Psychologist 2000;14:119-37.

71. Driediger M, Hall C, Callow N. Imagery use by injured athletes: a qualitative analysis. J Sports Sci 2006;24:261-71.

72. Simmons L, Sharma N, Baron JC, Pomeroy VM. Motor imagery to enhance recovery after subcortical stroke: who might benefit, daily dose, and potential effects. Neurorehabil Neural Repair 2008; 22: 458-67.

73. Braun SM, Beurskens AJ, Dalemans R, Schols JM, Wade DT. The experience of movement imagery therapy by patients with stroke or Parkinson's disease. (Submitted 2010) 



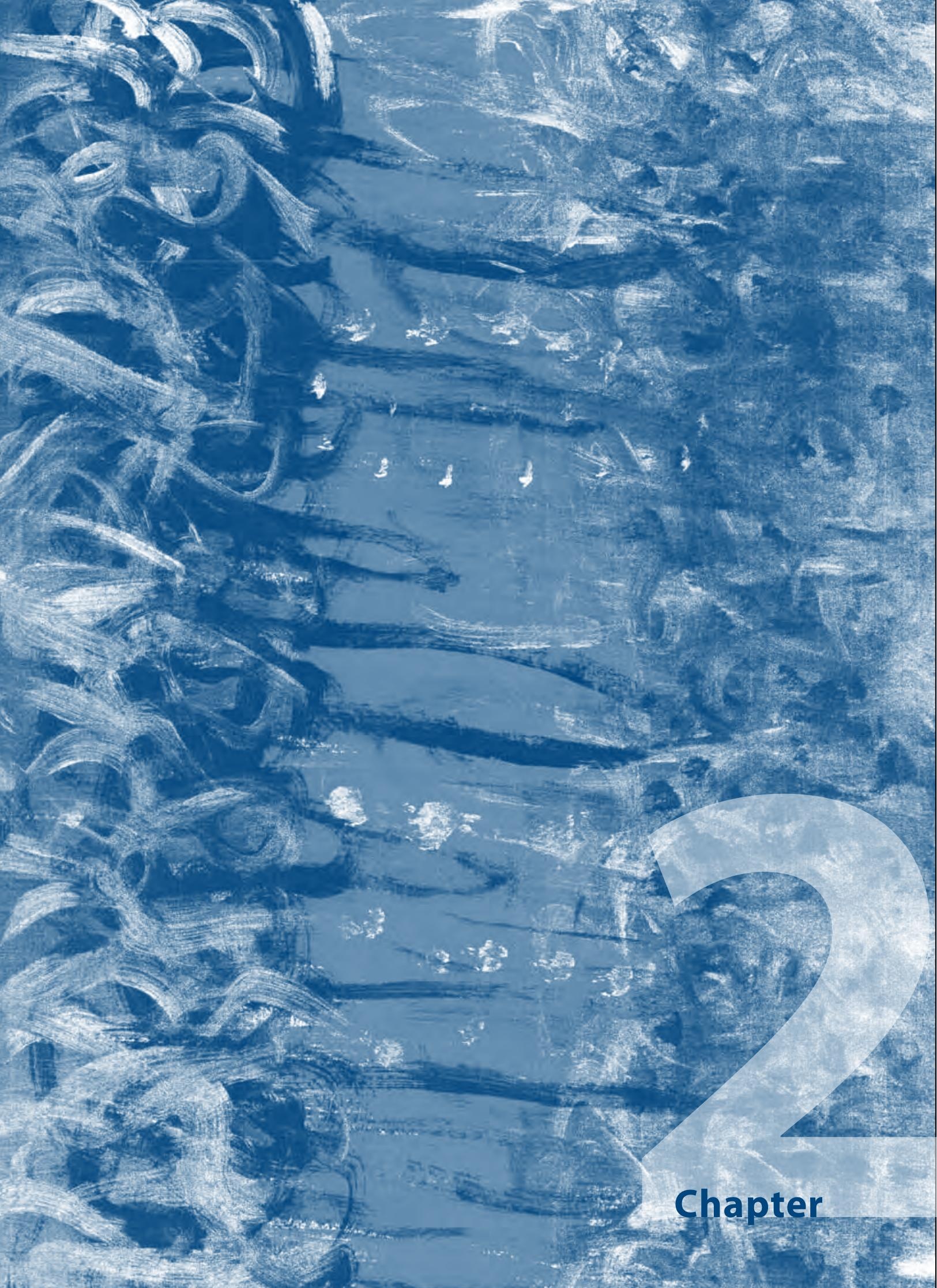
ii. 


\section{Chapter two}

The effects of mental practice in stroke rehabilitation: a systematic review

Braun SM, Beurskens AJ, Borm PJ, Schack T, Wade DT.

Arch Phys Med Rehabil 2006;66:842-852 


\section{Abstract}

Objective: To assess the effects of a mental practice intervention on recovery in stroke patients.

Data sources: A systematic literature search of the Cochrane Library, PubMed/Medline, PsycINFO, Pedro, Rehadat, and RehabTrials was performed by 2 researchers independently. Eligible studies published up to September 2005 were selected.

Study selection: Four randomised controlled trials (RCTs), 1 controlled clinical trial (CCTs), 2 patient series, and 3 case reports that investigated the effects of a mental practice intervention on recovery of stroke patients were included.

Data extraction: The selected RCTs and CCT were assessed on a methodologic quality rating scale. Important characteristics and outcomes were extracted and summarized. Results and characteristics from the patient series and case reports were only provided if they added information.

Data synthesis: Included studies differed clearly from each other with regard to patient characteristics, intervention protocol, and outcome measures. Four different mental practice strategies were used. Most tasks involved mentally rehearsing movements of the arm. Intervention periods varied from 2 to 6 weeks, frequencies ranged from multiple sessions per day to 3 times a week. Studies were limited in size. Power could not be increased by pooling or meta-analysis because studies were not comparable. Three of the 4 RCTs were of reasonable methodologic quality: there was some evidence that mental practice as an additional therapy intervention had positive effects on recovery of arm function after stroke. Two mental practice techniques seemed to be effective, tape instruction and self-regulation. Results from the single case studies indicate that mental practice also seemed promising for improvement of leg function.

Conclusions: No definite conclusions could be drawn except that further research using clear definitions of the content of mental practice and standardized measurement of outcome are needed. 


\section{The effects of mental practice in stroke rehabilitation: a systematic review}

\section{Introduction}

Stroke is a very limiting disease for the patient and a major health problem in most parts of the world $(1,2)$. It is therefore important to keep searching for new therapy techniques to improve recovery. Recently, imagery and mental practice have become additional therapy interventions $(3,4)$.

Studies in the first half of the last century suggested that mental execution of tasks resulted in improved performance in simple motor tests (5). Later studies were primarily conducted in different fields of sport psychology (6). In sports, the subject of imagery is movement and the main aim of movement imagery is to enhance specific motor actions (7). Studies that examine the effects of mental practice in sport frequently use 3 or 4 testing groups: a control group that receives no therapy, and at least 2 experimental groups, one of which practices mentally and the other physically. A fourth group may practice both mentally and physically and it is usually anticipated that the most substantial effects will be found in this group. Feltz and Landers $(8,9)$ reviewed studies in which all 4 groups (control group, physical practice, mental practice, mental and physical practice) were included, but inclusion criteria were broad and mental practice contents not always comparable. Perhaps because of this diversity in compared studies the results were remarkable: the performance of the group that trained only physically was greater than the performance of both the mental practice and the mental and physical practice groups. Other meta-analyses $(6,8)$ did not involve an explicit comparison with a group that combined mental and physical practice. In general however, it is apparent that mental practice has positive effects on performance enhancement (effect size range, .21-.68) $(6,8)$. Several studies in sports psy-

chology have shown that if mental practice is used as the only training form, it can improve the execution of movements in individual athletes and help in the acquisition of new skilled behaviors (10-16). But these effects may be less significant than those of physical exercise or physical exercise combined with mental practice (6).

Generalizing from sportsmen to disabled people is not necessarily possible, and the plausibility of mental practice in rehabilitation is at present based primarily on the results of brain imaging studies and small studies with stroke patients (17-31). Positive experiences reported from these studies stress the need to evaluate this therapy form further as an additional rehabilitation method. 
Although reviews, meta-analysis and critical overview articles on this subject have been published in the field of sports psychology $(6,8,9,32-37)$, there has been no systematic review on the effects of mental practice within stroke rehabilitation (38). In this review, we assessed systematically the potency of mental practice for rehabilitation purposes. More specifically, our objective was to undertake a systematic review primarily of randomised (RCTs) and non-randomised controlled studies (non-RCTs) that investigated the effects of a mental practice intervention in improving recovery in stroke patients. Results of other types of studies are reported if they provided additional information on the subject.

\section{Methods}

Figure one (next page) provides an overview of the search strategy, selection criteria, and assessment of the quality of the selected studies.

\section{Data sources}

In the literature, the terms movement imagery, motor imagery, and mental practice are used to describe different aspects of cognitive functions $(4,8,17)$. To increase the readability of this review we used only the term 'mental practice' for which the following working definition was used: During mental practice an internal representation of the movement is activated and the execution of the movement repeatedly mentally simulated, without physical activity, within a chosen context. It is used for the goal-oriented improvement or stabilization of a given movement.

The computer-supported search used the following data bases: Cochrane Library, PubMed/ Medline, PsycINFO, Pedro, Rehadat, and Rehabtrails. The strategy we developed for searching PubMed/Cochrane was used as the main protocol, and then modified for other sources. Modification was necessary because of the often more limited search options of these electronic databases. The key words used were: imagery, rehabilitation, physical therapy, activities of daily living, psychomotor performance, and stroke. The detailed search strategy is available from the authors.

The reference lists of the articles we have included were searched to trace potential further studies. Because there is a limited number of research groups investigating the effects of mental practice on recovery, we also did searches on the authors of any selected study.

All sources were searched independently by three investigators (SMB [researcher], MJ [librarian], KO [researcher]). 


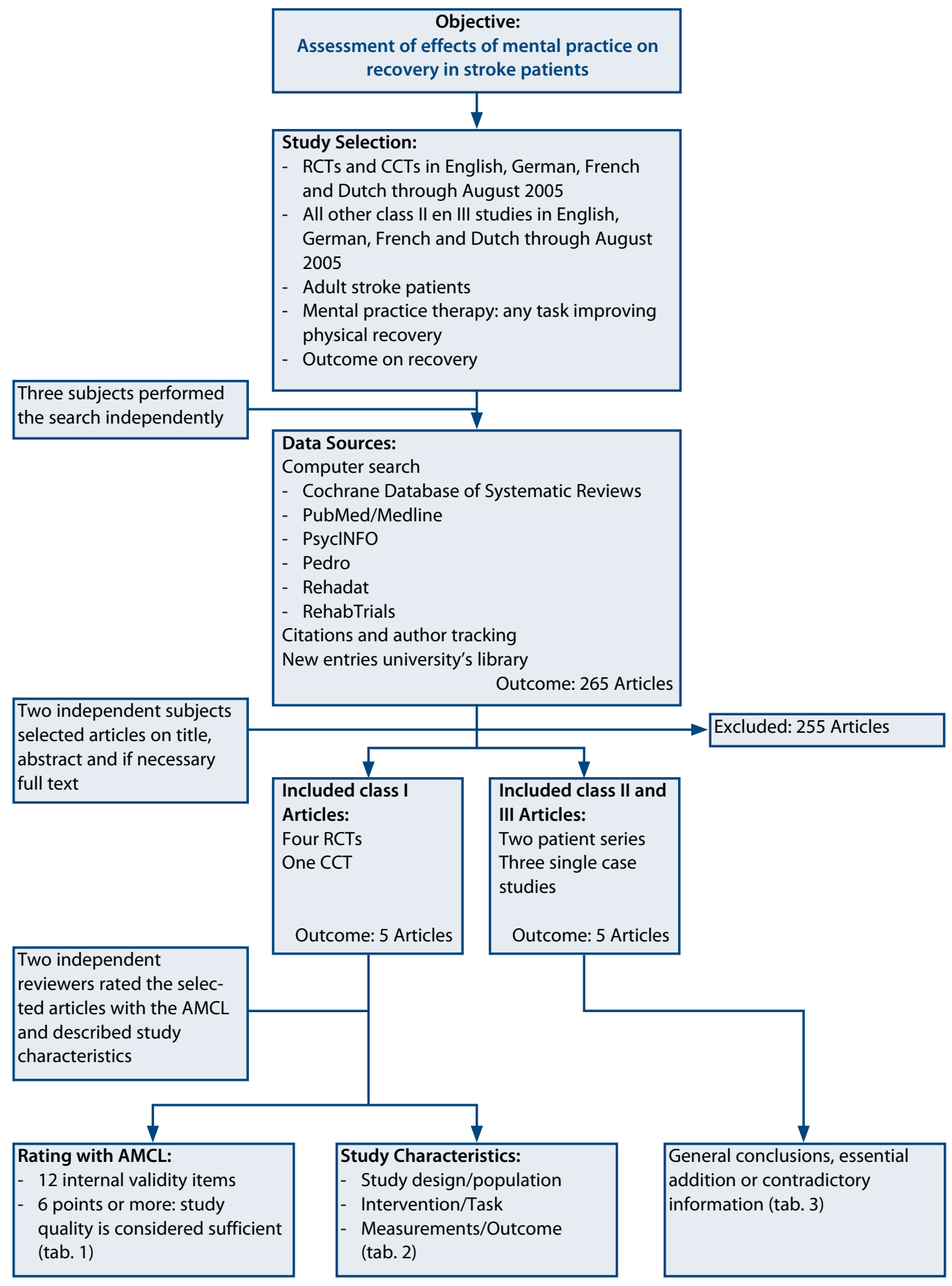

Fig. 1: Overview of literature search, criteria and rating.

Abbreviations: AMCL, Amsterdam-Maastricht Consensus List for Quality Assessment; RCT, randomised clinical trial; CCT, controlled clinical trial. 


\section{Study selection}

Types of study. The studies included in the review were all available articles in English, German, French, and Dutch through August 2005 that reported the effects of mental practice to improve physical recovery during the rehabilitation of adult stroke patients. The articles were categorized in classes I to III, with class I being RCTs and controlled clinical trials (CCTs), class II being cohort studies, and class III being single cases as well as noncontrolled patient series (39). Our focus was on the class I studies: RCTs and CCTs that investigated the relative efficacy of treatments. Articles from the other classes are described in less detail, but general conclusions and essential additional and contradictory information from the studies are reported to provide a more complete overview of the existing evidence on effects of mental practice in stroke rehabilitation.

Types of tasks and interventions. The interventions that were included could take place separately from physical therapy (PT) (e.g. using a taped instruction), be embedded in rehabilitation therapy (e.g. problem-solving strategies in which overt movements are combined with mental practice during PT) or given as the only therapy intervention. Interventions that required the use of specific equipment, however, such as electro-myographic stimulation and feedback and forms of virtual reality with computer simulation, were excluded.

Types of outcome measures. Outcome measures were divided into categories according to the international classification of the World Health Organization of function, activity, and participation (International Classification of Functioning, Disability and Health [ICF-2]) (40). The ICF-2 is a practical tool with which to elicit and record information on a subject's functioning and disability. For patients it is important that interventions reduce limitations on activities, for example in walking, drinking, or writing, and/or limitations on social participation, such as being able to continue in the role of cook. Therefore, improvements should preferably not be restricted to change in impairment only. Studies were excluded if the imagery was used merely for enhancing performance on a psychologic level like controlling anxiety or increasing motivation. If these skills were practiced in addition to physical performance, the studies were included (fig. 1, p 19).

\section{Data extraction}

The screening of the abstracts was done by two authors (SMB, MJ). The eligibility criteria regarding type of study, participants, tasks, and intervention were used to select relevant studies (fig. 1). If, based on the information in the abstract, it was not clear whether the study should be included, the full text of the article was assessed. Consensus was used to resolve disagreements concerning inclusion of studies and a third reviewer (AJB) was consulted if disagreement persisted. 
Methodologic quality of the RCTs and CCTs. To rate quality, we used 12 internal validity items from the Amsterdam-Maastricht Consensus List for Quality Assessment (AMCL) (fig. 1, p 19 and tab. 1, p 23) (41-43). The AMCL includes all criteria of other prominent quality scales such as the Delphi list $(44,45)$. Each criterion was scored either positive (+, 1 point), negative $(-, 0$ points), or unclear (?, 0 points), leading to a maximum score of 11 points per study ( 1 point for the items 2 to $11 ; 1 / 2$ point for the items $1 \mathrm{a}$ and $1 \mathrm{~b})$. Two reviewers (SMB, AJB) independently assessed the methodologic quality of the RCTs and CCTs. The articles were not blinded because the researchers were familiar with the literature.

To reduce differences in the assessment of the quality of the selected studies resulting from different interpretation of the 12 items beforehand, the validity criteria were defined and then discussed by the two researchers. For example, it was necessary to define 'acceptable' in 'an acceptable percentage of dropouts' (criterion 7). It was set at $10 \%$ during the intervention period and also at $10 \%$ from the remaining sample during follow-up, as suggested by Bonsel (46) and van Tulder (43) and colleagues. Compliance (criterion 5) was considered sufficient if patients themselves or therapists and relatives reported that the participants followed the given instructions. A follow-up period (criterion 11) of at least 6 months was considered clinically relevant for this intervention. For the last two criteria (criteria 5, 11) reviews of other interventions within health care were used as standard, because generally accepted references in the literature were not found $(43,45)$. Each item of the AMCL was then explained in a separate appendix that provided uniform operationalization of criteria (fig. 1, tab. 1).

We defined a study as being of 'sufficient quality' if the score was equal to or above 6 points $(42,43,45)$. Again, because standard references from the literature are missing, we defined the cut-off after references from other reviews were taken into account.

Study characteristics of RCTs and CCTs. The most important study characteristics were extracted by two independent reviewers $(\mathrm{SMB}, \mathrm{AJB})$ in order to investigate possible associations with the studies' outcomes. We developed a data extraction form for this specific review and disagreement between the reviewers about the characteristics were resolved before data were extracted. The following characteristics were extracted: study design and population, intervention and task, and measurements and outcome (fig. 1, tab. 2, p 26). 


\section{Results}

Two hundred sixty-five articles were identified in the Cochrane Database of Systematic Reviews (reviews, $n=126$; Database of Abstracts of Reviews of Effectiveness, $n=8$; Cochrane Central Register of Controlled Trials, n=14; National Health Service Economic Evaluation Database, n=2), PubMed/Medline ( $n=94)$, PsycINFO ( $n=12)$, and Pedro ( $n=9)$. We identified 22 topic-related research programs in RehabTrials $(n=16)$, and Rehadat $(n=6)$. The last two sources, RehabTrials and Rehadat, provided information about programs of ongoing research into the effects of mental practice interventions. Each of those research programs listed references, which we searched for leads on articles that might be included. There were none.

The first selection was based on title, abstract, and key words. In addition to theoretical articles, we excluded articles of effect studies because (1) participants were not primarily diagnosed with stroke or had other (additional) severe pathology (e.g. dementia, cancer, heart failure, depression, epileptic insults, Parkinson's disease, human immunodeficiency virus, trauma), (2) the intervention involved forms of virtual reality (e.g. mirror therapy) or use of medication, or (3) the purpose of the mental practice was other than to increase physical recovery (vision, speech, sleep, challenging behavior), or (4) a combination of the above. This process resulted in 17 retrieved articles of which four were double references (47-59). Three of the remaining 13 articles were excluded after the full text was read (52-54). The feasibility study by Malouin et al (53) was excluded because intervention time consisted only of a 1-hour session. The second article (52) was a fundamental study on the chronometry of imagined and overt movements, again measured after a single intervention session. The study by Stevens and Stoykov (54) involved use of computer-generated movies and mirror box-facilitated imagery and was excluded. Ultimately, five class I studies met the eligibility criteria (47-51) as well as five class III studies, of which two were patient series (55, 56), and three were single-case reports (57-59). The final class I selection included five studies: four RCTs $(47,48,50,51)$ and one CCT (49). Study sizes were small (four studies had $<20$ participants, one study had 46 patients (50)). In four of the five studies, only the effects of mental practice on recovery of arm function were investigated $(47-49,51)$. In one study the emphasis was on arm function but other skills were trained as well (50). Three of the five class III studies aimed at improving arm function (55-57), two investigated improvement of a mental practice intervention on the leg and foot $(58,59)$. Study sizes varied from one (57-59) to 10 (55) subjects.

\section{Effects and methodologic quality of class I studies}

The overall scores, as well as scores on each item on the AMCL for each study separately, are shown in table 1 . Overall scores varied from 2.5 to 7. 
Tab. 1: Quality assessment of internal validity of the selected clinical and RCTs with the Amsterdam-Maastricht Consensus List for quality assessment (41).

\begin{tabular}{|c|c|c|c|c|c|}
\hline Items & $\begin{array}{l}\text { Page } \\
(45)\end{array}$ & $\begin{array}{l}\text { Page et al } \\
\qquad(46)\end{array}$ & $\begin{array}{c}\text { Dijkerman et } \\
\text { al (47) }\end{array}$ & $\begin{array}{l}\text { Liu et al } \\
\text { (48) }\end{array}$ & $\begin{array}{c}\text { Page et al } \\
(49)\end{array}$ \\
\hline 1a Randomization & + & + & - & + & + \\
\hline $1 \mathrm{~b}$ Concealment of allocation & $?$ & + & - & + & + \\
\hline $\begin{array}{l}2 \text { Comparable subgroups at } \\
\text { base line }\end{array}$ & - & - & + & + & $?$ \\
\hline 3 Blinded care provider & $?$ & + & $?$ & - & $?$ \\
\hline $\begin{array}{l}4 \text { Correction for attention: } \\
\text { same treatment (dose), } \\
\text { co-intervention }\end{array}$ & + & + & - & + & + \\
\hline 5 Acceptable compliance & $?$ & + & + & $?$ & $?$ \\
\hline 6 Blinded patient & - & - & - & - & + \\
\hline $\begin{array}{l}7 \text { Acceptable withdrawals } \\
\text { during intervention period }\end{array}$ & $?$ & + & + & + & + \\
\hline 8 Blinded outcome assessor & $?$ & + & $?$ & + & + \\
\hline 9 Relevance measures & - & + & + & + & + \\
\hline 10 Timing assessment & - & - & - & - & - \\
\hline 11 Intention to treat analysis & $?$ & $?$ & - & $?$ & $?$ \\
\hline Total & 2.5 & 7 & 4 & 6 & 6 \\
\hline
\end{tabular}

NOTE. Range 0 to 11 points.

Legend: +, 1 point;,- 0 points; ?, 0 points.

Four of the five studies had positive scores on randomization procedures (item 1a $(47,48,50,51)$ ) of which three reported concealment of allocation (item $1 \mathrm{~b}(48,50,51)$ ). In the CCT, participants were assigned to a group to assure comparable subgroups at baseline (49) (positive score on item 2).

Items 3, 6, and 8 cover the aspects of blinding of the care provider (item 3), blinding of the patient (item 6), and blinding of the measurement assessor (item 8). Four of the five studies scored negatively on blinding of the care provider and all studies scored negatively on blinding of the patient. The care provider was blinded only in Page et al (48), in which the mental practice intervention was given by tape. Blinded assessors were reported in three studies $(48,50,51)$. Because mental practice was given as an additional intervention in four of the five studies (47, $48,50,51)$, it is of importance to correct therapy time and attention in the control group for additional therapy time and attention given to the experimental group. All four studies scored positively on this criterion (item 4). If mental practice was embedded in the occupational therapy (OT) or PT, as in Liu et al (50), it was hard to estimate the precise amount of additional attention. 
In Dijkerman et al (49) no intervention was given in the second control group, and therefore there was no correction for given attention (negative score on item 4).

Acceptable compliance was reported in two studies (item 5). Page et al (48) and Dijkerman et al (49) used logs and phone calls half way through the program to check on compliance with the program.

All studies scored negatively on the timing of the assessment criterion (item 10). The intervention periods were relatively short- and long-term effects were not measured.

Three of the five studies scored 6 points or more and were considered to have sufficient methodologic quality $(48,50,51)$. All three showed results in the same positive direction for performance enhancement of arm function only $(48,51)$ or arm function in combination with other skills (50) (tab. 2). There was significant improvement in the recovery of the patients in two studies at both the 'impairment' and 'activity' level $(48,51)$. One study of low quality showed positive effects of a mental practice intervention on physical recovery at the level of impairment only (47). The other study of low quality revealed no positive effects of the mental practice intervention on physical functioning beyond those the control group (49).

\section{Effects of class III studies}

All five class III studies showed positive effects of a mental practice intervention on physical recovery of arm (55-57) and leg functions $(58,59)$. The following additional positive effects were reported: decrease in performance time (gait speed improved (58)), and increased consistency in performance and decreased response time (foot-sequence task (59)).

\section{Effects in relation to patient characteristics of class I studies}

Patients in the studies were recruited through local advertisements in therapy clinics and hospitals $(47,48,51)$, the stroke database at the local hospital (49) and by asking hospital inpatients (50) to consider participation. Inclusion (49-51) or exclusion criteria $(47,48)$ were described in all five studies. In one study (49), inclusion was restricted to one criterion, the ability to perform a motor task to some extent with the affected arm. In other studies, inclusion contained restrictions concerning age, recovery stage, cognitive and sensory abilities, communication skills, spasticity and pain, type and location of lesion, and the ability to image. The time between stroke and start of therapy varied from seven days to six years. Acute, sub acute, post acute, and chronic phase of recovery were not defined uniformly in the separate studies.

The classification used by the authors of the articles is shown in table two. In three studies, subjects in the chronic phase of recovery were recruited or patients had to have stable motor deficits $(47,49,51)$. Liu et al (50) included exclusively patients in the sub acute phase of recovery (7-27d post stroke). Page et al (48) included patients in the chronic as well as acute phase of recovery (4wk to 4y post stroke). Only male subjects participated in the study by Page (47). 
All other studies included both men and women. The average age varied from 62.3 (51) to 72.7 years (50). In general, the patient characteristics of these studies were not associated with better or worse outcomes (tab. 2).

\section{Effect in relation to patient characteristics of class III studies}

The characteristics of the participants in the class III studies were similar to those of the RCTs and CCT, except that younger participants were involved, in which positive effects of mental practice on recovery were also identified $(55,59)$ (tab. 3).

\section{Effect in relation to intervention characteristics class I studies}

There were three major strategies within the mental practice intervention: tape instruction $(47,48,51)$, self-regulation (50), and observation followed by practice (49).

In the three studies by Page et al $(47,48,51)$, instructions were given by tape and the mental practice session took place directly after PT in a separate room or was completed by the patient at home. Only in the Page study (47) was the content of the mental practice sessions individually tailored to the content of the PT: the task that was mentally rehearsed was chosen from the tasks that were trained physically in therapy.

In the study of Liu (50), the intervention was given by a therapist who taught the patient principles of problem solving and self-regulation in combination with imagery processes during the first week. This was the only study in which a training phase was included in the intervention period and the mental practice was actually first taught before it was implemented. Mental practice and overt movement were combined during the entire intervention period. In Dijkerman et al (49), the participants performed only mental training at home (experimental group and control group 1) or had no training at all (control group 2). They were asked to perform the mentally trained task as an overt movement as well, but no guided physical training took place. It is not clear how instructions were given.

The interventions varied, from drinking from a cup to 15 functional tasks including household, cooking, and shopping tasks. In four studies (48-51), the tasks were defined before hand and standardized for all patients. In these studies, except for that of Dijkerman et al (49), the content changed from week to week during the intervention period. The same task was repeated during the entire intervention period.

The frequency with which the guided mental practice was given varied, but a minimum of two times a week for 20 minutes was reported in every study. Unguided mental practice training ranged from multiple sessions per day to three times a week. The intervention period varied from three to six weeks.

Because of the limited studies and heterogeneity of the intervention protocols used, the intervention characteristics could not be associated with the outcome. 


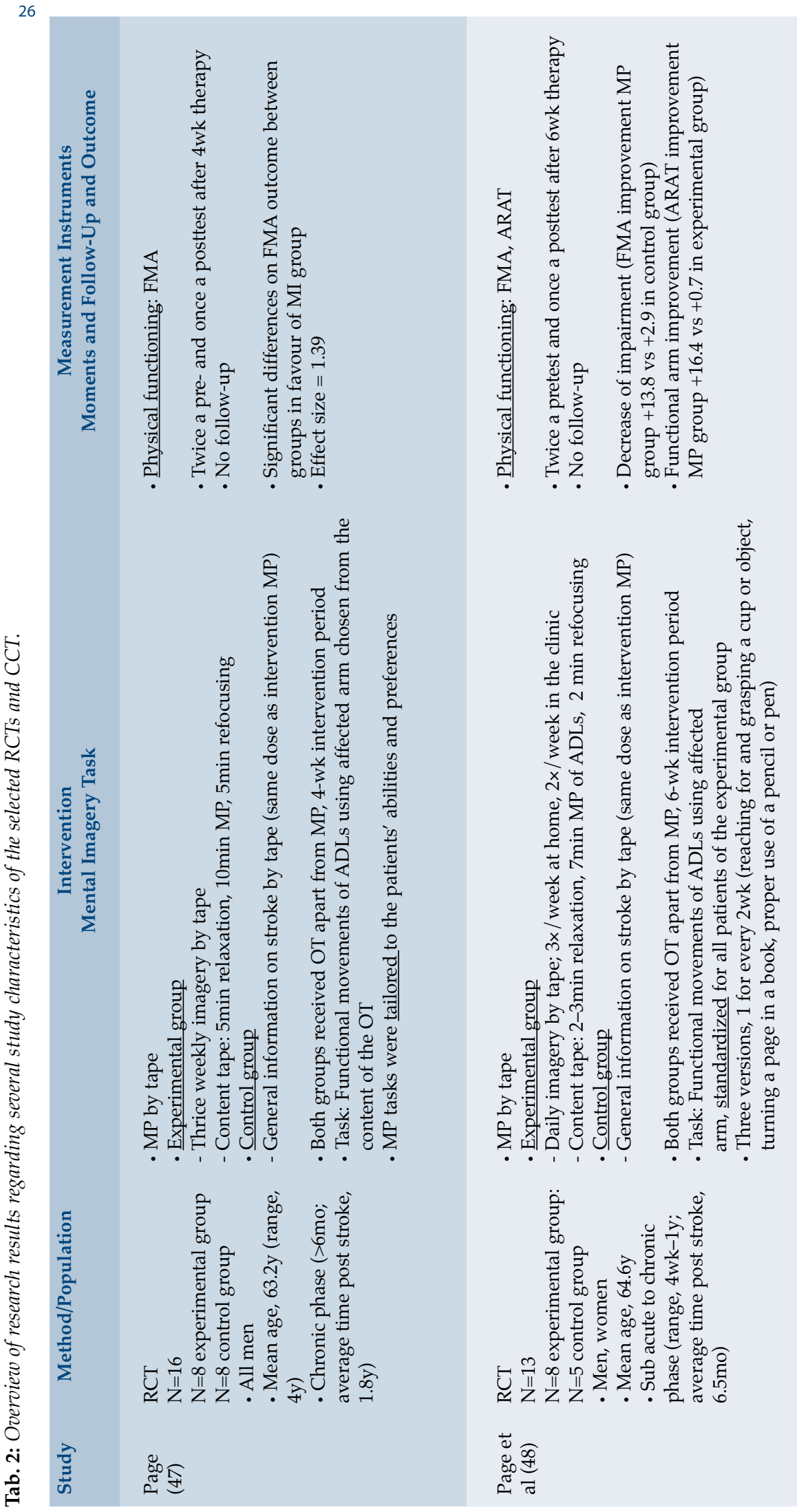



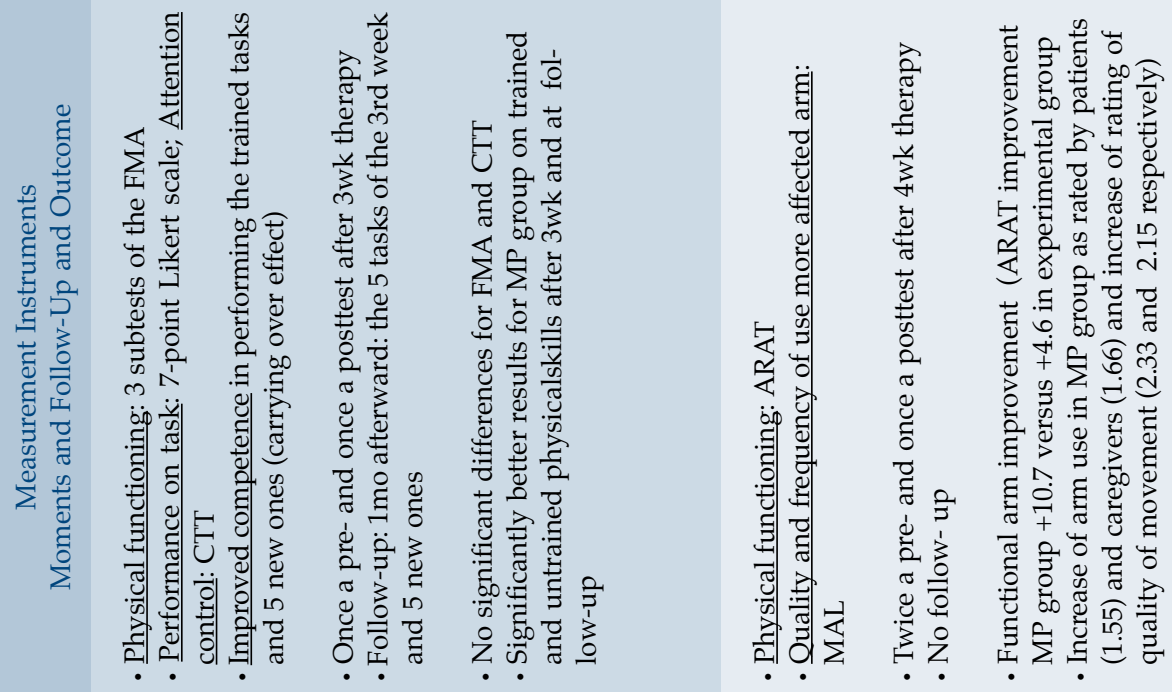

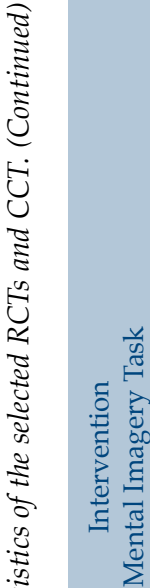

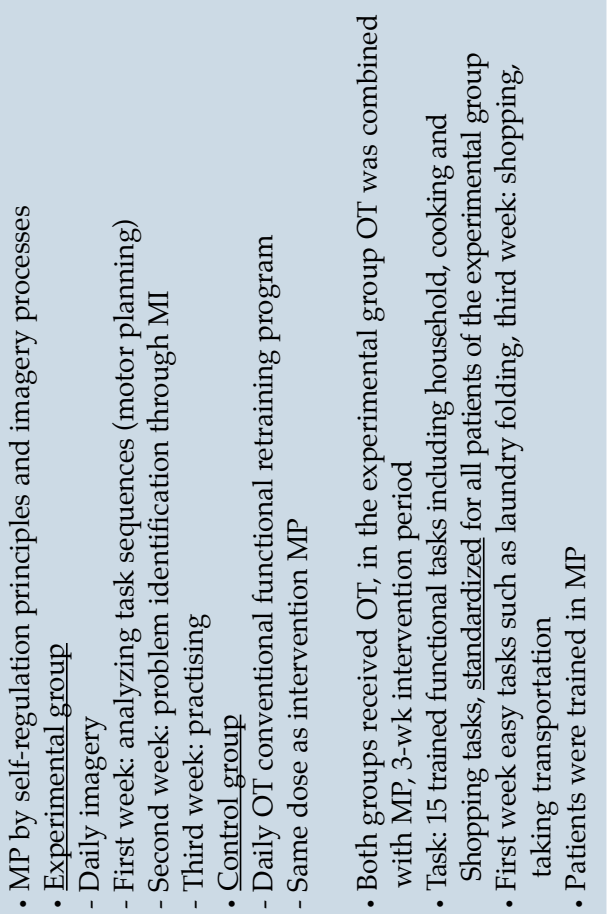

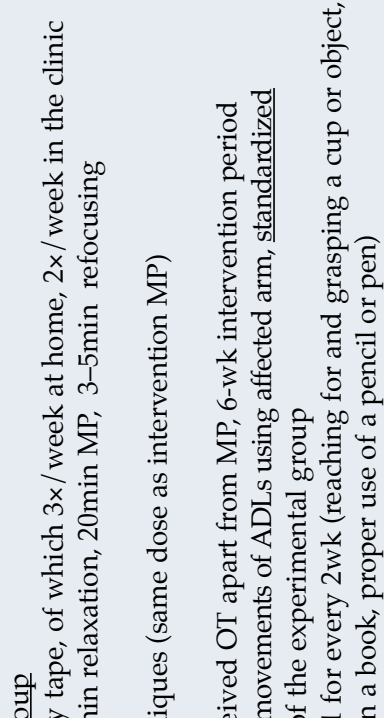
के

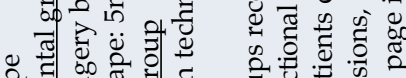

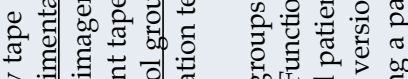

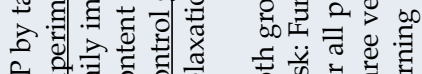

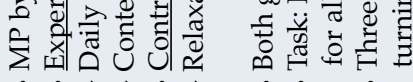

$\frac{5}{5}$ 


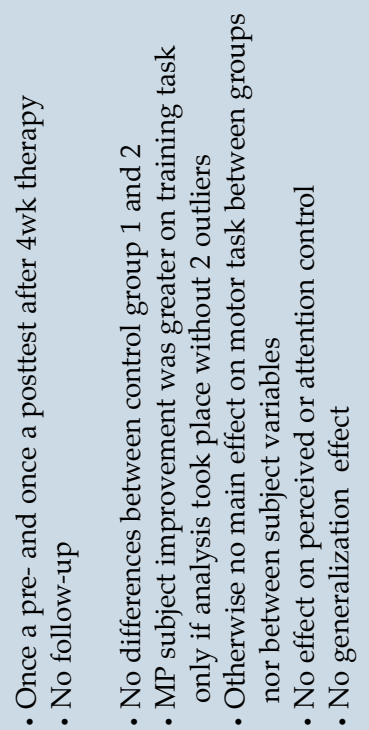

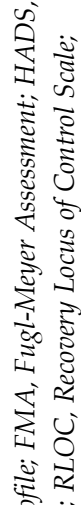

放 $\frac{7}{5}$

壳

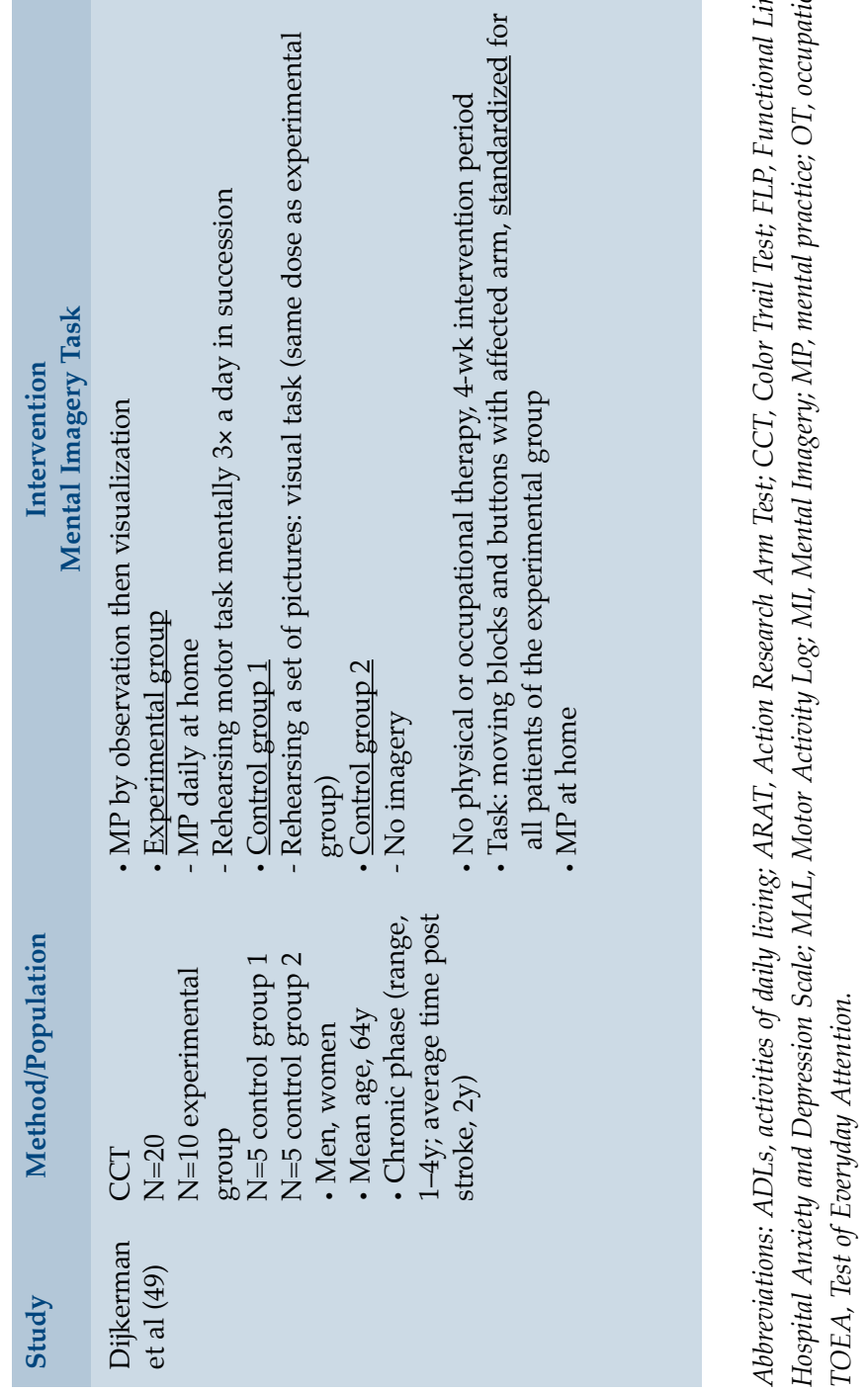




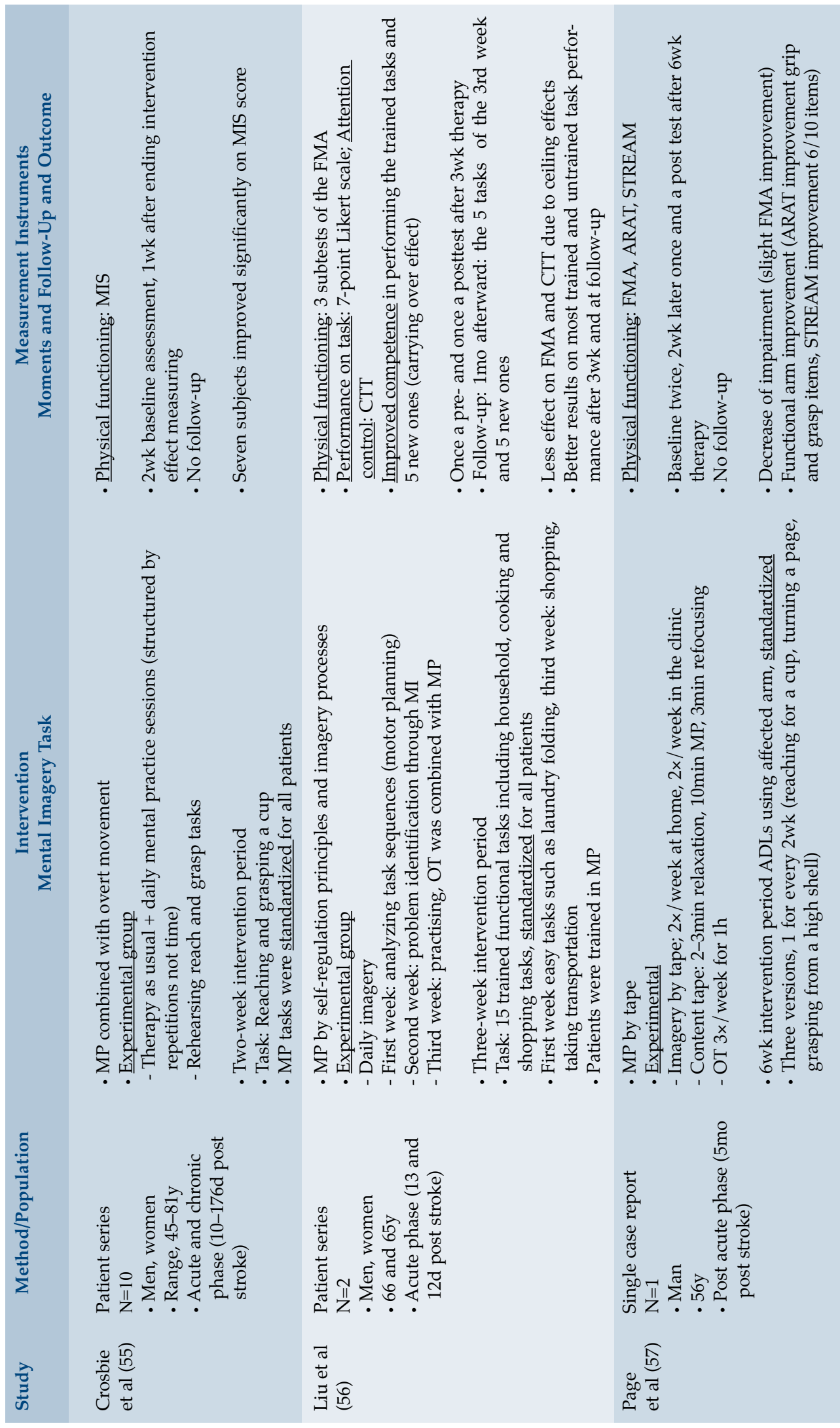




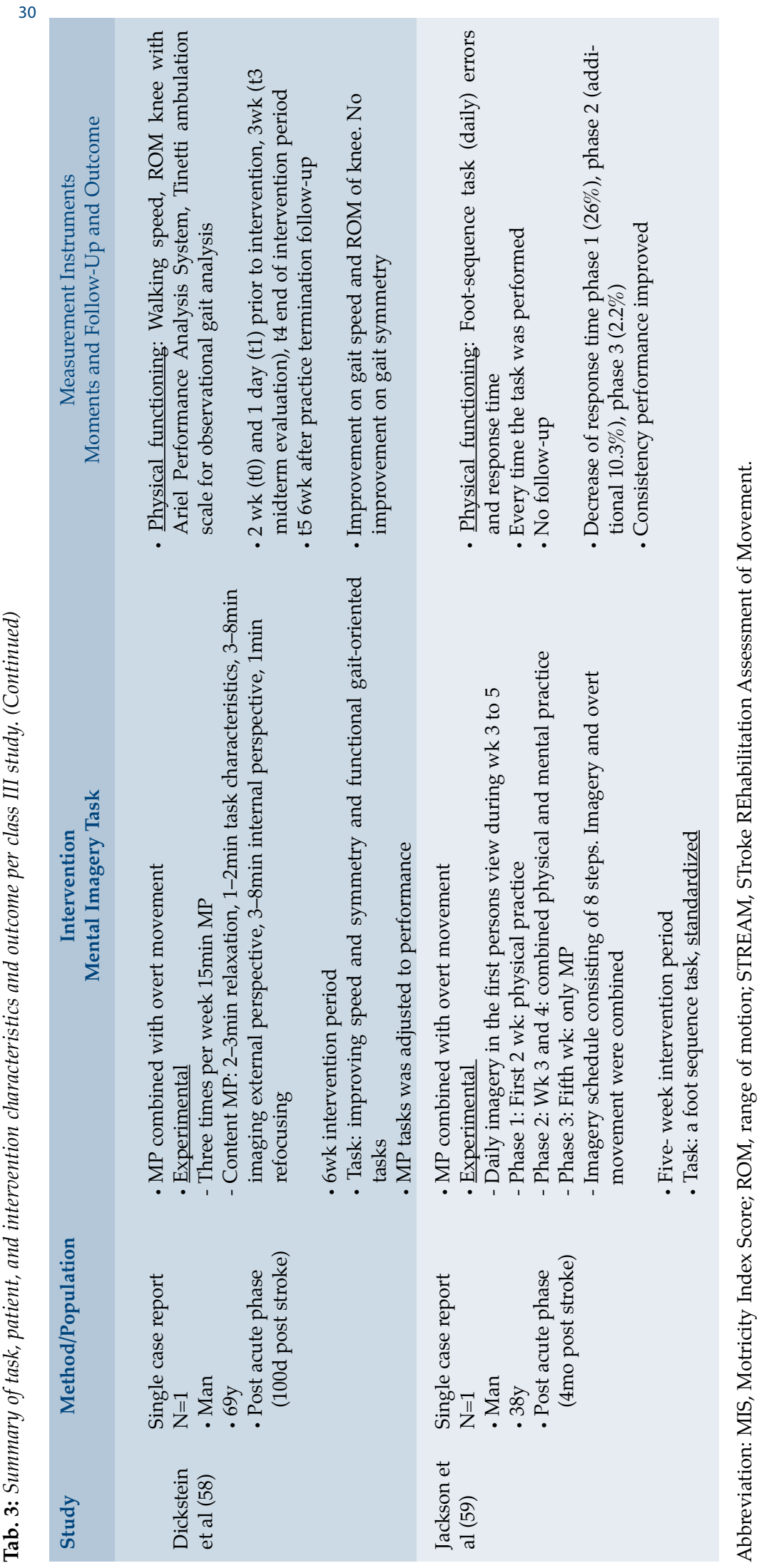




\section{Effect in relation to intervention characteristics of class III studies}

Although two of the five Class III studies were performed by researchers who were also included in the class I studies $(56,57)$, the three remaining added more variety to the intervention characteristics mentioned above. An additional potential strategy was described: 'mental practice in first or third view combined with overt movement' during therapy sessions $(55,59)$ or apart from it (58). In the third view, the patient sees him/herself perform the task as if looking from a distance. In the first view, the patient sees him/herself perform as if looking through his/ her own eyes. In Dickstein (58) and Jackson and colleagues (59), the intervention content seemed to be successfully expanded to tasks involving the legs and feet (gait, foot-sequences, respectively). Also, even short intervention periods of two weeks, as in Crosbie et al (55), seemed to have positive effects on arm function recovery (tab. 3).

\section{Discussion}

This systematic review has found that there is some evidence that mental practice as an additional therapy has effects on recovery after stroke. We identified relatively few studies that investigated the effects of mental practice. The nature of the mental practice intervention varied greatly. Moreover the outcomes measured varied a lot both in nature (measurement domain and instruments) and timing (recovery phase, moments of measurement). Meta-analysis was not possible (32), and no definite conclusions could be drawn, except that further research using clear defini-tions of the content of mental practice and standardized measurement of outcome, is needed.

\section{Effects and methodologic quality of class I studies}

It is remarkable that four of five heterogeneous class I studies with little power (n range, 11-46) showed significant effects on different domains of mainly arm function. The results from class III studies underline the results found in the four RCTs and CCT for arm function. In addition, there seemed to be positive effects of mental practice on leg function $(58,59)$.

Three of five class I studies had sufficient methodologic quality. Most studies did not report blinding of the patients or therapists, for active participation of the patient and instructions by the caregivers are often required. Only Liu et al (50) included a follow-up after one month, therefore little is known about possible long-term effects of mental practice. Reports on compliance were poor, but in most studies attrition was low, which can be an indication that patients were able to comply with the treatment content (48-51). Correction for attention and therapy intensity was provided in almost all included studies $(47,48,50,51)$. Effects of the experimental intervention reported in this review are therefore more likely contributed to mental practice. 


\section{Effects in relation to patients characteristics}

The patient characteristics in the included studies differed with regard to inclusion criteria, age, phase of recovery, sex, and affected side and dominant side of the arm. In several studies, minimal physical, communication and attention skills were required for admission. The studies indicated that both men and women with acute and chronic hemiplegia of left and right sides could possibly benefit from mental practice.

\section{Effect in relation to intervention characteristics}

We found that the available evidence was restricted to mental practice by tape recording (47, 48,51 ) and mental practice embedded in PT or OT, including self-regulation, problem-solving techniques, and imaging processes (50). There was no evidence that mental practice without any form of guided PT or OT enhanced motor performance (49), which is in line with results of sports psychology research (6) (tab. 2).

In the study of Liu et al (50), there also seemed to be some evidence of generalization effects of mental practice during and directly after a three week intervention period. Generalized principles of the effects of mental practice on untrained physical skills were not found at one month follow-up. Apparently, the learned problem solving and self-regulation principles could not be maintained when patients had to apply them without guidance.

In just one class I study (47) the practiced task was directly retained from the physical therapy content. In other words, the task was also physically trained in therapy and then used as content for the mental practice session. In several studies the chosen task was 'reaching for or drinking from a cup'. One may doubt whether a unimanual task, which can be performed with the nonaffected arm, or a task that is not chosen by the patient, is in practice sufficiently motivational for him/her to comply with mental practice, although in the selected studies it did not appear to affect compliance negatively. An exception was the study by Page et al (51), in which the intervention was aimed at reducing non-use of the more affected arm both in the experimental and control group. Unimanual tasks of the affected arm are then, of course, necessary.

Doses and intensities of a mental practice intervention varied considerably in the studies. Studies with healthy subjects found that the longer someone mentally practices without combining the task with overt movements, the less beneficial it becomes (6). Linking physical activities and mentally performed movements appears essential for effective implementation. Perhaps that is why in the study by Dijkerman et al (49), there was no apparent improvement on arm function in the mental practice group. Linking the appropriate kinesthetic information a movement generates directly to the mental practice can be achieved by combining mental practice techniques and overt movements in one training protocol $(50,53,55)$. 
In summary, there is neither consensus within the studies on the content of the intervention protocol nor the intervention dose. The intervention strategies clearly differed. There is no evidence that one intervention protocol was more effective than another if mental practice and overt movement were somehow regularly combined.

\section{Conclusions}

\section{Methodologic aspects of this review}

As with any review article, our conclusions are subject to some common points of criticism concerning the standardized rating of the methodological quality of the controlled trials with the original protocol of the AMCL. First, blinding of patients is impossible if a cognitive strategy within therapy is assessed, for patients are asked to actively think about, influence and alter their behavior. In other studies, such as van Tulder et al (42) this criterion was redefined in order to make it possible to score positive and increase the study's quality. We decided to maintain the original rating definitions because assessing methodologic quality should be objective and standardized instead of altered to the limitations of the field of research.

Second, the setting of cut-offs and percentages was arbitrary, although examples in other studies were considered.

Third, missing values in the text were not retrieved by contacting the authors or research groups. If the information was not in the article, or if it was not clear whether certain procedures were followed, the criterion was scored negative or with a question mark. Information not reported is not necessarily information not retrieved and therefore the criteria list assesses the study report, not necessarily the quality of the study.

Systematic reviews, including this one, also probably miss some studies because of the limitations of searching algorithms and the categorization of studies with databases. For example, the intervention may not be described as imagery or mental practice but as a cognitive or behavioral intervention. Because of the inconsistent use of different terms, such as motor imagery, mental practice, and movement imagery, it was necessary to read the entire article to understand what exactly had been done with the patient during the experimental phase. Furthermore, mental practice could not be used as a key word because it is not listed in the thesaurus as a search term.

One should also take a possible publication bias into account. Small studies with a negative outcome are difficult to get published. 


\section{Implications for practice}

If mental practice strategies involve active participation of the patient during the intervention (not only passively undergoing it) the technique should first be taught $(50,56)$. Transferring the technique into schematic cognitive strategies might help the patient applying mental practice during rehabilitation (6). One should consider incorporating a training period at the start of the intervention period.

Little is known about the long-term effect of mental practice interventions. It seems however that unguided mental practice should be combined with the overt movement and refreshment trainings should be implemented on a regular basis, thereby linking kinesthetic information to mental representations. Thus, mental practice might be more suitable for patients still receiving PT or OT (60).

\section{Acknowledgments}

We thank Marsha Jussen and Kenneth Oti for their contributions to the literature search.

\section{References}

1. American Heart Association. Stroke facts. Dallas: AHA; 1997.

2. Commissie CVA-revalidatie. Revalidatie na een beroerte, richtlijnen en aanbevelingen voor zorgverleners. Den Haag: Nederlandse Hartstichting; 2001.

3. Leeuwen van R, Inglis J. Mental practice and imagery: a potential role in stroke rehabilitation. Phys Ther Rev 1998;3:47-52.

4. Jackson PL, Doyon J, Richards CL, Malouin F. Potential role of mental practice using motor imagery in neurological rehabilitation. Arch Phys Med Rehabil 2001;82:1133-41.

5. Sackett RS. The influence of symbolic rehearsal upon the retention of a maze habit. J Gen Psychol 1934;10:376-95.

6. Driskel JC, Copper C, Moran A. Does mental practice enhance performance? J Appl Psychol 1994;79:481-92.

7. Boschker M. Action-based imagery. On the nature of mentally imagined motor actions [dissertation]. Amsterdam: Vrije Universiteit Amsterdam Ipskamp PrintPartners; 2001.

8. Feltz DL, Landers D. The effects of mental practice on motor skill learning and performance: a meta-analysis. J Sport Psychol 1983;5:25-57.

9. Feltz DL, Landers D. A revised meta-analysis of the mental practice literature on motor skill learning and performance. In: Druckman D, Swets J, editors. Enhancing human performance: issues, theories and techniques. Washington (DC): Natl Acad Pr; 1988. p 1-65.

10. Gould D, Damarjian N, Greenleaf C. Imagery training for peak performance. In: Raalte van J, Brewer WB, editors. Exploring sport and exercise psychology. Washington (DC):

Am Psychol Assoc; 2002. p 49-74. 
11. Hale BD. Imagery perspectives and learning in sports performance. In: Sheikh AA, Korn ER, editors. Imagery in sports and physical performance. Farmingdale: Baywood; 1994. p 75-96.

12. Hall C. Imagery and the acquisition of motor skills. Can J Sports Sci 1992;17:19-27.

13. Janssen JJ, Sheikh AA. Enhancing athletic performance through imagery: an overview. In: Sheikh AA, Korn ER, editors. Imagery in sports and physical performance. Farmingdale: Baywood; 1994. p 1-22.

14. McCullagh PW, Weiss M. Observational learning: the forgotten psychological method in sport psychology. In: Raalte van J, Brewerz WB, editors. Exploring sport and exercise psychology. Washington (DC): Am Psychol Assoc; 2002. p 131-50.

15. Meyers AW, Cooke CJ, Cullen J, Liles L. Psychological aspects of athletic competitors: a replication across sports. Cogn Ther Res 1979;3:361-6.

16. Williams JM, Leffingwell TR. Cognitive strategies in sport and exercise psychology. In: Raalte van J, Brewer WB, editors. Exploring sport and exercise psychology. Washington (DC): Am Psychol Assoc; 2002. p 75-98.

17. Decety J. The neurophysiological basis of motor imagery. Behav Brain Res 1996;77:45-52.

18. Mellit E, Petit L. Reopening the mental imagery debate: lessons from functional anatomy. Neuroimage 1998;8:129-39.

19. Nair DG, Purcott KL, Fuchs A, Steinberg F, Kelso JA. Cortical and cerebellar activity of the human brain during imagined and executed unimanual and bimanual action sequences: a functional MRI study. Brain Res Cogn Brain Res 2003;15:250-60.

20. Decety J, Grezes J, Costes N, et al. Brain activity during observation of actions. Influence of action content and subject's strategy. Brain 1997;120:1763-77.

21. Decety J, Kawashima R, Gulyas B, Roland PE. Preparation for reaching: a PET study of the participating structures in the human brain. Neuroreport 1992;3:761-4.

22. Decety J, Perani D, Jeannerod M, et al. Mapping motor representations with positron emission tomography. Nature 1994;371:600-2.

23. Hallett M, Fieldman J, Cohen LG, Sadato N, Pascual-Leone A. Involvement of primary motor cortex in motor imagery and mental practice [comment]. Behav Brain Sci 1994;17:210.

24. Harris DV, Robinson WJ. The effects of skill level on EMG activity during internal and external imagery. J Sport Psychol 1986;8:105-11.

25. Johnson SH. Imagining the impossible: intact motor representations in hemiplegics. Neuroreport 2000;11:729-32.

26. Jackson PL, Lafleur MF, Malouin F, Richards CL, Doyon J. Functional cerebral reorganization following motor sequence learning through mental practice with motor imagery. Neuroimage 2003;20:1171-80.

27. Jacobson E. Electrophysiology of mental activities. Am J Psychol 1932;44:677-94. 
28. Malouin F, Richards CL, Jackson PL, Dumas F, Doyon J. Brain activations during motor imagery of locomotor-related tasks: a PET study. Hum Brain Mapp 2003;19:47-62.

29. Weiss T, Hansen E, Beyer L, et al. Activation process during mental practice in stroke patients. Int J Psychophysiol 1994;17:91-100.

30. Trombly CA, Wu CY. Effect of rehabilitation tasks on organization of movements after stroke. Am J Occup Ther 1999;53:333-44.

31. Malouin F, Potvin M, Prevost J, Richards CL, Wood-Dauphinee S. Use of an intensive taskoriented gait training program in a series of patients with acute cerebrovascular accidents. Phys Ther 1992;72:781-93.

32. Budney AJ, Murphy SM, Woolfolk RL. Imagery and motor performance: what do we really know? In: Sheikh AA, Korn ER, editors. Imagery in sports and physical performance. Farmingdale: Baywood; 1994. p 97-120.

33. Corbin CB. Mental practice. In: Morgan WP, editor. Ergogenic aids and muscular performance. New York: Acad Pr; 1972. p 94-118.

34. Grouios G. Mental practice: a review. J Sport Behav 1992;15:42-59.

35. Richardson A. Mental practice: a review and discussion (part I). Res Q 1967;38:95-107.

36. Richardson A. Mental practice: a review and discussion (part II).Res Q 1967;38:263-73.

37. Martin K, Moritz S, Hall C. Imagery use in sports: a literature review and applied model. Sports Psychol 1999;13:245-68.

38. Bell AR, Murray BJ. Improvement in upper limb motor performance following stroke: the use of mental practice. Br J Occup Ther 2004;11:501-7.

39. Cicerone KD, Dahlberg C, Malec JF, et al. Evidence-based cognitive rehabilitation: updated review of the literature from 1998 through 2002. Arch Phys Med Rehabil 2005;86:1681-92.

40. Wade DT, Halligan PW. Do biomedical models of illness make for good healthcare systems? BMJ 2004;329:1398-1401.

41. Bouter LM, van Dongen MC. Systematisch literatuuronderzoek. In: Bouter LM, van Dongen MC, editors. Epidemiologisch onderzoek. Opzet en interpretatie. Houten/Diegem: Bohn Stafleu Van Loghum; 2000. p 361-87.

42. van Tulder MW, Ostelo RW, Vlaeyen JW, Linton SJ, Morley SJ, Assendelft WJ. Behavioral treatment for chronic low back pain: a systematic review within the framework of the Cochrane Back Review Group. Spine 2001;26:270-81.

43. van Tulder M, Furlan A, Bombardier C, Bouter L. Updated method guidelines for systematic reviews in the Cochrane collaboration back review group. Spine 2003;28:1290-9.

44. Verhagen AP, de Vet HC, de Bie RA, et al. The Delphi list: a criteria list for quality assessment of randomized clinical trials for conducting systematic reviews developed by Delphi consensus. J Clin Epidemiol 1998;51:1235-41. 
45. Huibers MJ, Beurskens AJ, Bleijenberg G, van Schayck CP. The effectiveness of psychosocial interventions delivered by general practitioners. Cochrane Database Syst Rev 2003(2): CD003494.

46. Bonsel GJ, Offringa M. Kwaliteit van leven. In: Offringa M, Assendelft WJ, Scholten RJ, editors. Inleiding in evidence-based medicine. Houtem/Diegem: Bohn Stafleu Van Loghum; 2000. p 119-30.

47. Page SJ. Imagery improves upper extremity motor functions in chronic stroke patients with hemiplegia: a pilot study. Occup Ther J Res 2000;20:200-15.

48. Page SJ, Levine P, Sisto S, Johnston MV. A randomized efficacy and feasibility study of imagery in acute stroke. Clin Rehabil 2001;15:233-40.

49. Dijkerman HC, Letswaart M, Johnston M, MacWalter RS. Does motor imagery training improve hand function in chronic stroke patients? A pilot study. Clin Rehabil 2004;18:538-49.

50. Liu KP, Chan CC, Lee TM, Hui-Chan CW. Mental imagery for promoting relearning for people after stroke: a randomized controlled trial. Arch Phys Med Rehabil 2004;85:1403-8.

51. Page SJ, Levine P, Leonard AC. Effects of mental practice on affected limb use and function in chronic stroke. Arch Phys Med Rehabil 2005;86:399-402.

52. Malouin F, Richards CL, Desrosiers J, Doyon J. Bilateral slowing of mentally simulated actions after stroke. Neuroreport 2004;15:1349-53.

53. Malouin F, Richards CL, Doyon J, Desrosiers J, Belleville S. Training mobility tasks after stroke with combined mental and physical practice: a feasibility study. Neurorehabil Neural Repair 2004;18:66-75.

54. Stevens JA, Stoykov ME. Using motor imagery in the rehabilitation of hemiparesis. Arch Phys Med Rehabil 2003;84:1090-2.

55. Crosbie JH, McDonough SM, Gilmore DH, Wiggam MI. The adjunctive role of mental practice in the rehabilitation of the upper limb after hemiplegic stroke: a pilot study. Clin Rehabil 2004;18:60-8.

56. Liu KP, Chan CC, Lee TM, Hui-Chan CW. Mental imagery for relearning of people after brain injury. Brain Inj 2004;18:1163-72.

57. Page SJ, Levine P, Sisto SA, Johnston MV. Mental practice combined with physical practice for upper-limb motor deficit in sub acute stroke. Phys Ther 2001;81:1455-62.

58. Dickstein R, Dunsky A, Marcovitz E. Motor imagery for gait rehabilitation in post-stroke hemiparesis. Phys Ther 2004;84:1167-77.

59. Jackson PL, Doyon J, Richards C, Malouin F. The efficacy of combined physical and mental practice in the learning of a footsequence task after stroke: a case report. Neurorehabil Neural Repair 2004;18:106-11.

60. van Peppen RP, Kwakkel G, Harmeling-van der Wel BC, et al. KNGF-richtlijn Beroerte. Ned Tijdschr Fysiother 2004;114(Suppl):1-77. 


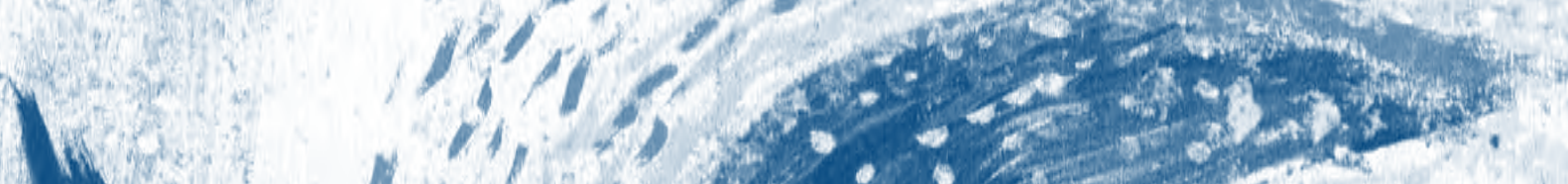

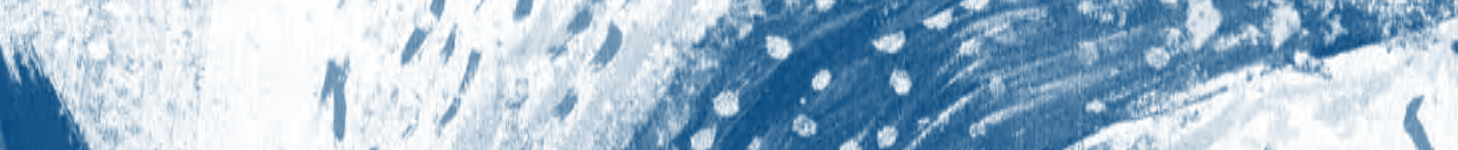
ifi: $20 .: 98=$ 11. II': (1) $10.1 \%$ W
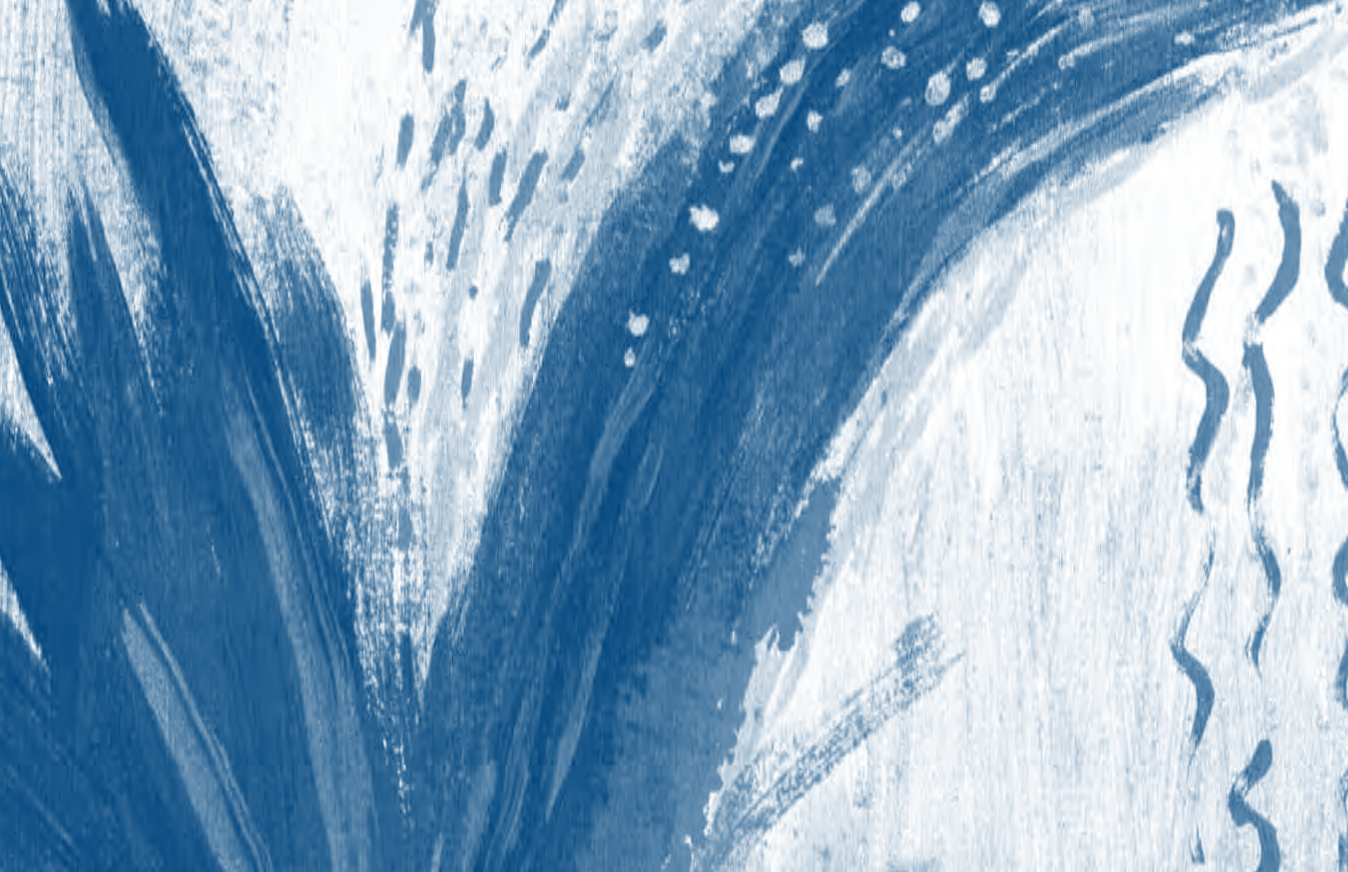

$1(2 y)$
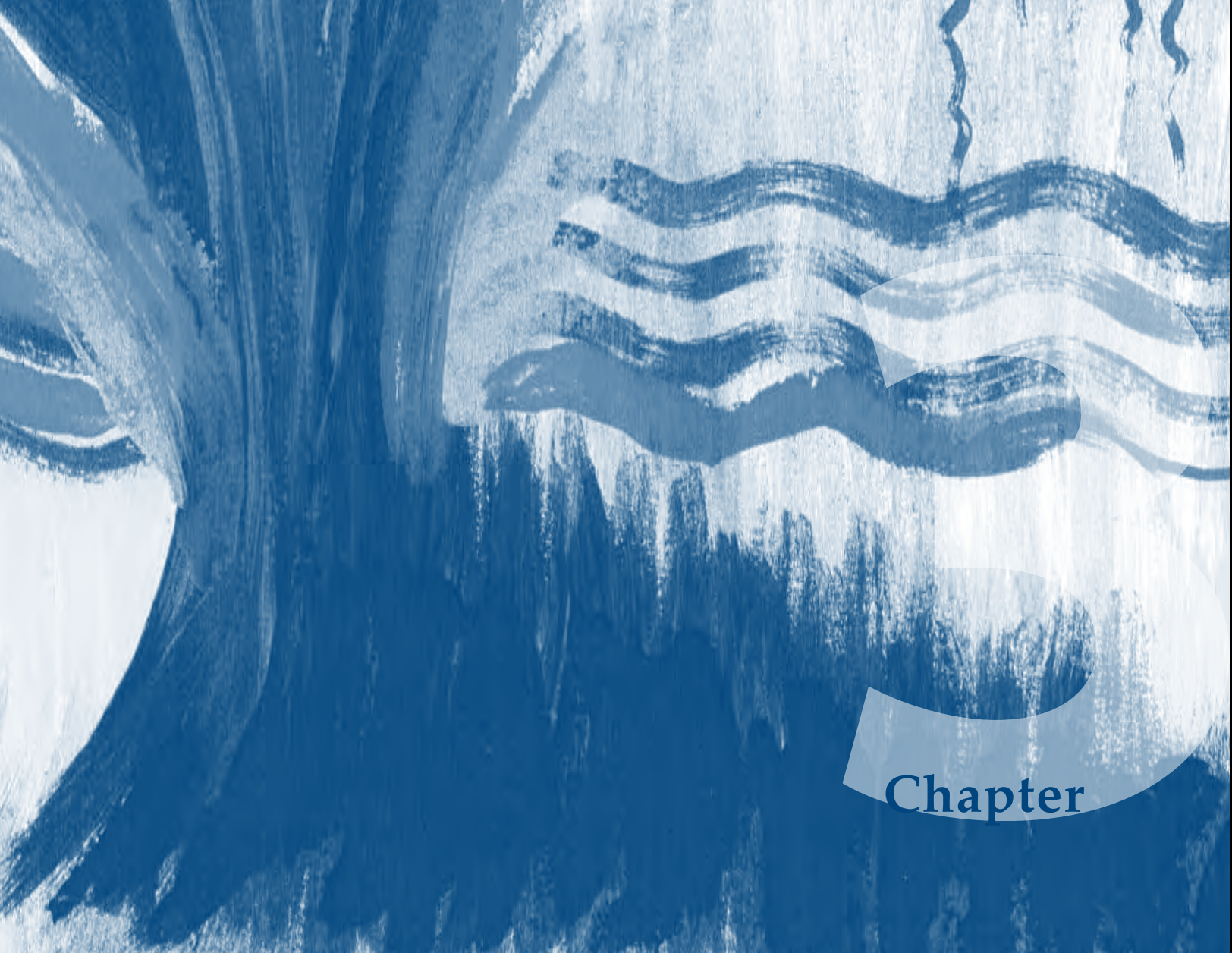

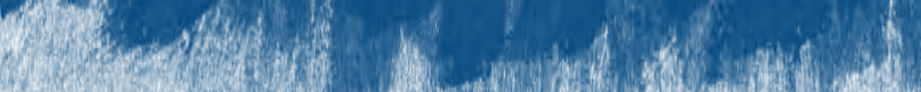




\section{Chapter three}

Is it possible to use the Structural Dimension Analysis of Motor memory (SDA-M) to investigate representations of motor actions in stroke patients?

Braun SM, Schack T, Marcellis RG, Oti KC, Schols JM, Beurskens AJ, Wade DT.

Clin Rehabil 2007;21:822-832 


\section{Abstract}

Objective: To determine the feasibility of the Structural Dimension Analysis of Motor memory (SDA-M), a method derived from sports psychology, in establishing the mental representations of complex movements in patients after stroke.

Design: Case series of patients, with age-matched healthy controls.

Setting: A rehabilitation nursing home.

Subjects: Sixteen patients 3-26 weeks after their stroke, and 16 controls.

Intervention: Each control had the SDA-M performed within a 10-day period. Each stroke patient had the SDA-M performed once. In the SDA-M the subject was asked to state for each of 10 actions involved in drinking from a cup whether it is functionally close to each of the other nine or not.

Main measures: The raw data from the SDA-M were transformed through cluster analysis into Euclidean distances and tree diagrams to illustrate the internal representation of the action.

Results: All subjects were able to perform the assessment. Healthy controls all had a similar set of Euclidean distances and tree diagrams that were 'normal'. The tree diagrams remained very similar on the three occasions. Four stroke patients had tree diagrams that were 'normal'. The remaining twelve had tree diagrams that differed greatly both from the 'normal' and from each other, with much less clustering of actions. Patients with more severe stroke appeared to have more disordered tree diagrams.

Conclusion: The Structural Dimension Analysis of Motor memory (SDA-M) is a feasible method for investigating the mental representation of internal motor action plans in stroke patients, giving similar data in stable healthy people and revealing abnormal patterns in patients after stroke. 


\section{Is it possible to use the Structural Dimension Analysis of Motor memory (SDA-M) to investigate representations of motor actions in stroke patients?}

\section{Introduction}

Planning and undertaking complex motor actions can be disrupted by many neurological conditions such as stroke. Failure to undertake complex motor tasks in a correct sequence and with correct relative timing may lead to problems, such as spilling hot coffee before the cup reaches the mouth. A better understanding of motor performance in the presence of neurological damage might lead to more effective rehabilitation treatments. This study investigated a method for establishing the patient's awareness of their planning of complex motor tasks as a prelude to developing a specific treatment protocol based on motor imagery (1).

The cognitive-perceptual hypothesis of central motor planning, derived from movement research $(2,3)$ and neurophysiology, (4-9) suggests that movement representations have the same spatio-temporal structure within the brain as the actual movement. During motor imagery the motor cortex becomes active in the absence of movement execution. This activity mainly consists of activation of mirror neurons in the prefrontal and parietal cortex and forms the basis of motor action understanding (10). Movement imagery and movement execution may be based on the same representation $(3,11-15)$.

A technique for investigating mental representation of movement in athletes, the Structural Dimension Analysis of Motor memory (SDA-M) has been developed and implemented successfully in different fields of sports such as volleyball, gymnastics and sky surfing $(14,16)$. The technique could possibly be used for any complex movement, such as the movements of a stroke patient having trouble drinking out of a cup.

As the SDA-M has not yet been published in the neurological literature, a short description of the tool will be given. Goal-directed movements are probably organized and stored in memory as 'events' by way of representation of their anticipated perceptual effects $(3,15)$. Schack and co-workers proposed the existence of 'basic action concepts' or 'motor representations' that 'can be considered as elementary components or transitional states of complex movement with specific functional and perceptual features'. Basic action concepts can be described verbally or through visual images. A few examples for drinking out of a cup are: opening the hand, moving the arm towards the cup, grasping the cup, etc. (tab. 1). 
In sport, the basic actions are selected and defined by experts and athletes based on common sense, observation and biomechanical analysis of the movement. It is assumed that they are stored in long-term memory and are moved into the working memory, being placed in the correct order and adapted to the biomechanical demands in a specific environment before being activated to perform the action.

Tab. 1: Basic Action Concepts for 'drinking out of a cup'.

$\begin{array}{ll}\text { Number } & \text { Basic Action Concept } \\ 1 & \text { Reaching for the cup } \\ 2 & \text { Grasping the cup } \\ 3 & \text { Lifting the cup } \\ 4 & \text { Bending the arm with the cup } \\ 5 & \text { Putting cup to lips } \\ 6 & \text { Tilting cup } \\ 7 & \text { Closing mouth and swallowing } \\ 8 & \text { Straitening arm with the cup } \\ 9 & \text { Putting cup down } \\ 10 & \text { Letting go of cup }\end{array}$

In sport, the SDA-M is used to determine the basic architecture of specific goal-directed movements: for example to identify weak spots in the sequence of events that should lead to a certain motor performance, such as a volleyball player having trouble with hitting a smash. (program at: www.unileipzig.de/_zander/split).

The SDA-M has not yet been tested in clinical settings. The objectives of this initial feasibility study were to:

1) investigate whether the SDA-M program can visualize the representation of 'drinking out of a cup' in healthy elderly subjects and if these results can be reproduced over time;

2) determine if there is a standard representation of 'drinking out of a cup' in the control group (similarity within the control group);

3) investigate whether the SDA-M program can visualize the representation of 'drinking out of a cup' in stroke patients; and if so to

4) compare the representations of the stroke group with that of the control group (to what extent do representations between the groups differ?). 


\section{Methods}

This study was approved by the medical ethical commission of the academic hospital of Maastricht, the Netherlands. For the evaluation of the SDA-M, healthy subjects were measured three times within a 10-day period (objectives 1 and 2). Patients were measured once only to determine differences within the stroke population and between the experimental and control group (objectives 3 and 4). Each measurement took about one hour. In a preliminary phase, the SDA-M as used in sport was simplified for use with people after stroke: the concepts were simplified and the information was offered both orally and visually (tab. 1, p 50 and fig. 1. p 53). Patients and healthy controls were measured with the same version of the SDA-M.

\section{Patient and control selection}

Patients were recruited from the stroke ward of the Klevarie nursing home of the Vivre foundation in Maastricht (in the Netherlands nursing homes deliver much stroke rehabilitation care). Patients had to meet following selection criteria:

- clinically diagnosed stroke;

- aged over 18 years;

- sufficient cognitive and communication ability and stamina to understand and carry out instructions for 1 hour. This was assessed clinically, usually through the patient completing the from the Dutch guidelines recommended neuropsychological screening test;

- no severe additional neurological, orthopaedic, rheumatoid or cardiac impairments.

Healthy controls were recruited from the partners of patients with stroke and from a local home for the elderly, matching for demographic statistics. The potential participants were informed orally and in writing. The nursing home physician of Klevarie recruited patients and the researchers recruited the healthy subjects. Both groups had at least two days to consider participation. They were included in the study after giving informed consent.

\section{Measurement protocol}

Before the first measurement, basic instructions were given to the subjects along with information on the project. Three measurements for the healthy subjects took place. Stroke patients were measured only once after a familiarization phase. Healthy subjects were asked to respond concerning their dominant arm: patients were asked to respond considering their affected arm. The complete task was shown in a video to ensure the subject understood which task (drinking out of a cup) had to be analysed, being displayed from an external (seeing someone performing the task) and/or internal view (as if looking through your own eyes), depending on the preference of the subject (18-20). 
The subject compared all the concepts (numbers 1-10, tab. 1), which were displayed at random on a computer screen next to each other, each time deciding whether they 'belonged together' or not. For instance, a photograph of 'holding the cup' (anchoring unit, basic action concept number 2, tab. 1 and fig. 1, p 45) is shown in the upper half of the screen and, in a random order the other nine actions are all presented underneath the anchoring unit. The subjects judged for each of the nine actions whether they were 'functionally close' to the anchoring photo above (yes or no).

\section{Statistical analysis}

As every action was used as an anchoring unit on the upper half of the screen, this procedure resulted in 10 decision trees per participant (we used 10 basic action components). The data were then analysed in four steps according to a hierarchical cluster analysis and factor analysis in order to determine the attractor state of the separate concepts $(3,19,21)$. The subjective distances between the mental representations of actions were made visible in a tree diagram (fig. 1). Possible variations in mental representations in the stroke population could therefore be detected by comparing these results with normative values derived from the control group. In addition, variations within the stroke population could be assessed.

The data were analysed according to a hierarchical cluster analysis and the results were displayed in a tree diagram. Information on how the tree diagram is statistically established can be obtained from the authors.

If two actions are often labeled by a subject as being strongly related, this is expressed in a small Euclidean distance resulting in a low projection of the basic action concepts on the vertical line in the tree diagram. If two actions are not judged to be strongly related and therefore not selected together during the splitting procedure, the Euclidean distance is long and the projection of the two actions is high in the tree diagram (fig. 1).

The measure of similarity between two tree diagrams can be calculated and is expressed in lambda. Lambda is a number, ranging from 0 (no similarity at all) to 1 (tree diagrams are identical). The cut-off point for stating that two diagrams are essentially the same is set at 0.68 (statistically significant: in this study the measuring fault, alpha, was set at $1 \%$ ).

Furthermore, individual results of tree diagrams were judged with common sense on several aspects:

- Is the clustering of the actions meaningful? In other words, can the movement organization lead to a successful performance?

- Are all actions integrated in the movement or a phase of the movement? 

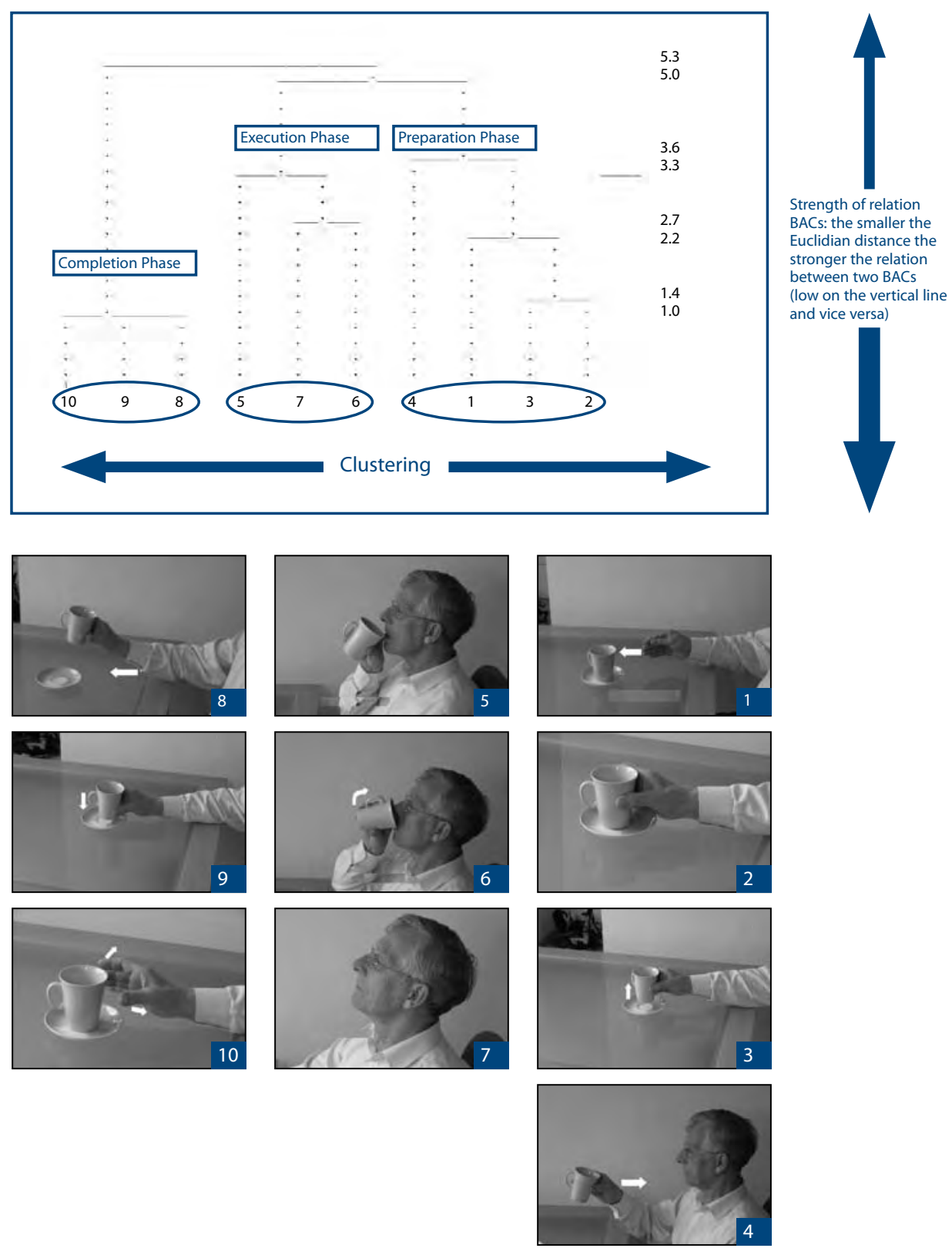

Fig. 1: Explanation for reading the tree diagram 'drinking from a cup': Average tree diagram of a healthy elderly subject in the control group. On the horizontal line the numbers of the basic action concepts (BACs) are represented as corresponding with the list shown in table 1. For instance, number 1 being 'reaching for the cup', number 7 being 'closing mouth and swallowing'. Early in the tree diagram one can distinguish the following clusters: one to four (preparation phase), five to seven (execution phase) and eight to 10 (completion phase). The most stable in the representation is the completion phase and the numbers two and three of the preparation phase for they are low on the vertical line. Number four 'Bending the arm with the cup' is clustered in the preparation phase but not imbedded firmly (high on the vertical line). The tree diagram is characterized by meaningful clusters (in phases) and strong(er) relations between the BACs. 
- Which actions relate strongly to each other?

- Are any of the actions related to a part of the movement in which they do not belong?

Moreover, does this affect the success of the movement performance?

With the above-described analytic procedure, the intra-individual differences in the measurements (T0, T1, T2) of the SDA-M over time in all healthy subjects were evaluated to assess the program's reproducibility.

Differences within the stroke group and healthy subject group were compared. Within the stroke group, inter-individual differences were calculated.

\section{Results}

Twenty-two patients following stroke were asked to participate in this study: three declinedparticipation and another three did not meet inclusion criteria. The remaining 16 subjectscompleted the measurement and all data could be used for analysis. In the healthy population 16 (of 17 approached) subjects were recruited for assessment. The characteristics of the samples are shown in table two.

Tab. 2: Patient characteristics at baseline.

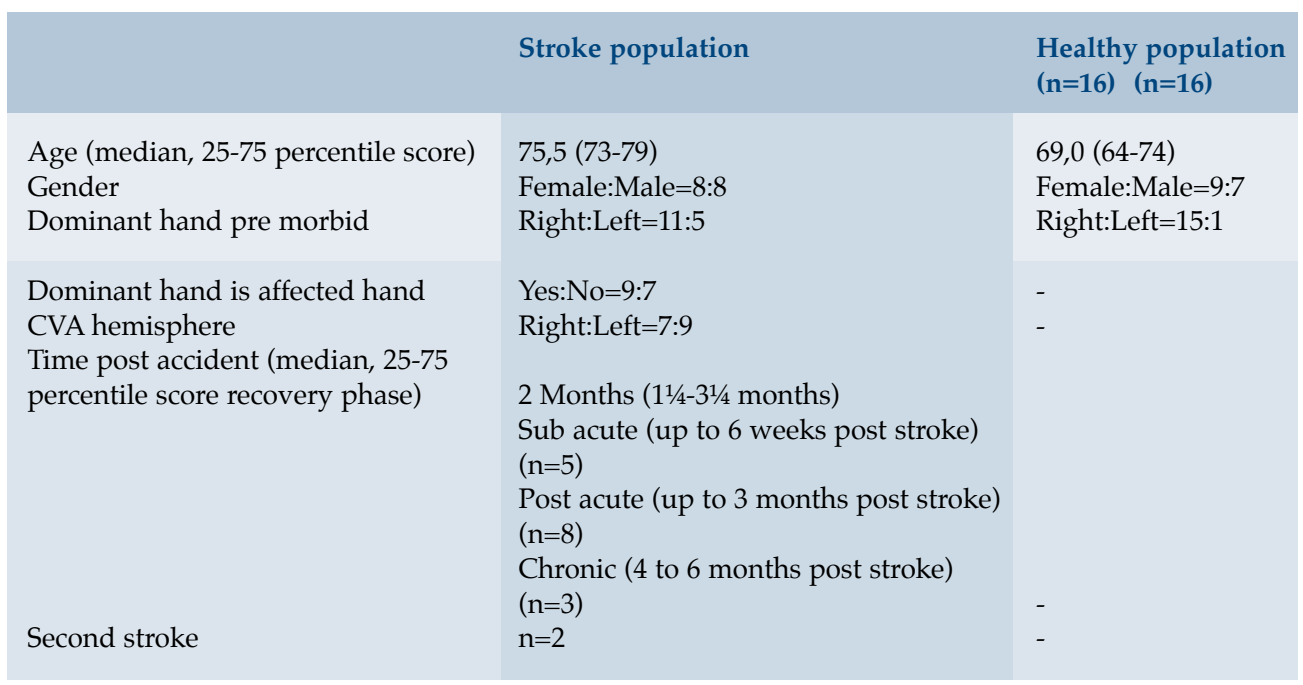

\section{Results within the healthy subject population}

The SDA-M was feasible to visualize mental representations of 'drinking out of a cup' within the control group. An example of a common representation of the task of one of the healthy subjects is given in figure one ( $\mathrm{p} 45)$. In 13 out of 16 subjects, we found a characteristic and logical clustering of actions in three phases: a preparation phase (numbers 1-4), an 
execution phase (numbers 5-7) and a completion phase (numbers 8-10). In the other three subjects, only two phases could be detected: an extended preparation and a combined execution/completion phase.

The actions that were judged as being the most related were the numbers 8-10. In general, the healthy subjects had great depth in their tree diagram, corresponding to a clear distinction between different clusters and their relation to each other. Healthy subjects had a clear and stable representation of their motor action. Furthermore, all actions were integrated into a phase (actions were seldom singled out) and actions were clustered logically.

Summarizing, the tree diagrams within the healthy group can be characterized by:

- large clusters: three or four basic action clusters;

- logical, meaningful phases: preparation, execution and completion;

- strong(er) relations between the actions, expressed in small Euclidean distances; and

- few actions not integrated into a specific phase or logical cluster structure: variations between subjects were limited.

When repeating the measurement within the control group the tree diagrams of a single subject were very similar. On average, the measure of reproducibility between the three measurements within the entire control group was $82 \%$ (lambda overall average control 0.816 ) and 85\% $(\lambda=0.852)$ when excluding the three subjects with only two phases in the tree diagram.

\section{Results within the stroke population}

Among the stroke patients two main subgroups could be identified. One subgroup of four patients had mental representations that resembled the average tree diagram of the control group. The larger subgroup ( $n=12)$ had tree diagrams that varied greatly, not resembling the normative tree diagram, nor showing any measure of similarity to others within the subgroup. The tree diagrams of stroke patients with a lesion in the right hemisphere had a smaller range (less depth) and greater Euclidean distances than the patients with a left hemisphere lesion. Table three ( $p$ 56) gives an overview of patient characteristics of the stroke patients.

\section{Patients with tree diagrams similar to the controls}

The four patients in this group had relatively minor motor and cognitive impairments affecting the task. Figure two ( $\mathrm{p} 49$ ) shows a tree diagram of a patient with a relatively normal representation but small yet characteristic differences remained:

- the actions were functionally not as close as in the control group and 
- there are more standalone actions that are not integrated into a specific phase (numbers 1,4 and 8). Although the clustering of the actions could still be seen as functional, the entire movement was sometimes represented in a more fragmented way. The least integrated actions were those describing the transition movements between the preparation and execution phase (number 4 'bending the arm with cup', significant) and the execution and completion phase (number 8 'straightening the arm with the cup') as well as the first action, 'reaching for the cup' (number 1).

Tab. 3: Overview of patient characteristics of the stroke patients with similar (nr. 1, 4-6) and different (nr. 2, 3, 7-16) representations compared to the blueprints of the healthy subjects.

\begin{tabular}{|c|c|c|c|c|c|c|c|c|c|}
\hline Nr. & $\begin{array}{l}\text { Age } \\
(y r)\end{array}$ & Gender & $\begin{array}{l}\text { Time post } \\
\text { stroke } \\
\text { (months) }\end{array}$ & $\begin{array}{l}\text { Affected } \\
\text { hemi- } \\
\text { sphere }\end{array}$ & $\begin{array}{l}\text { Dominant } \\
\text { hand }\end{array}$ & $\begin{array}{l}\text { Dominant } \\
\text { hand is } \\
\text { affected }\end{array}$ & $\begin{array}{l}\text { Education } \\
\text { level }\end{array}$ & $\begin{array}{l}\text { Mentally } \\
\text { rehearsed } \\
\text { imaging view }\end{array}$ & Note \\
\hline 1 & 76 & Female & 3,5 & Left & Right & Yes & High & Third person & \\
\hline 4 & 79 & Male & 2 & Right & Right & No & High & $\begin{array}{l}\text { No use of } \\
\text { imagery }\end{array}$ & \\
\hline 5 & 78 & Male & $3 / 4$ & Left & Right & Yes & Low & Third person & \\
\hline 6 & 85 & Male & 3 & Left & Right & Yes & High & Third person & \\
\hline 2 & 68 & Female & 4 & Left & Left & No & High & Third person & $\begin{array}{l}\text { Second } \\
\text { stroke }\end{array}$ \\
\hline 3 & 72 & Female & 3 & Right & Left & Yes & Low & Third person & \\
\hline 7 & 81 & Male & 5 & Right & Right & No & Low & Third person & $\begin{array}{l}\text { Second } \\
\text { stroke }\end{array}$ \\
\hline 8 & 75 & Male & 6 & Left & Right & Yes & High & First person & $\begin{array}{l}\text { Nonaffected } \\
\text { hand } \\
\text { became } \\
\text { dominant }\end{array}$ \\
\hline 9 & 74 & Female & 2 & Right & Left & Yes & Low & Third person & Neglect \\
\hline 10 & 73 & Female & $31 / 4$ & Left & Right & Yes & High & First person & $\begin{array}{l}\text { Non- } \\
\text { functional } \\
\text { arm }\end{array}$ \\
\hline 11 & 73 & Female & $1 / 2$ & Left & Left & No & Low & First person & \\
\hline 12 & 44 & Female & 1 & Right & Right & No & High & First person & \\
\hline 13 & 87 & Male & $3 / 4$ & Right & Right & No & Low & Third person & Apraxia \\
\hline 14 & 79 & Female & $13 / 4$ & Left & Left & Yes & Low & Third person & \\
\hline 15 & 70 & Male & 2 & Right & Right & No & Low & First person & \\
\hline 16 & 84 & Male & $1^{1 / 4}$ & Left & Right & Yes & Low & Third person & \\
\hline
\end{tabular}

Education level: Low: up to high school; High: college and higher or training on the job Imaging view: First person: as if looking through his/her own eyes | Third person: as if looking at oneself from a distance 


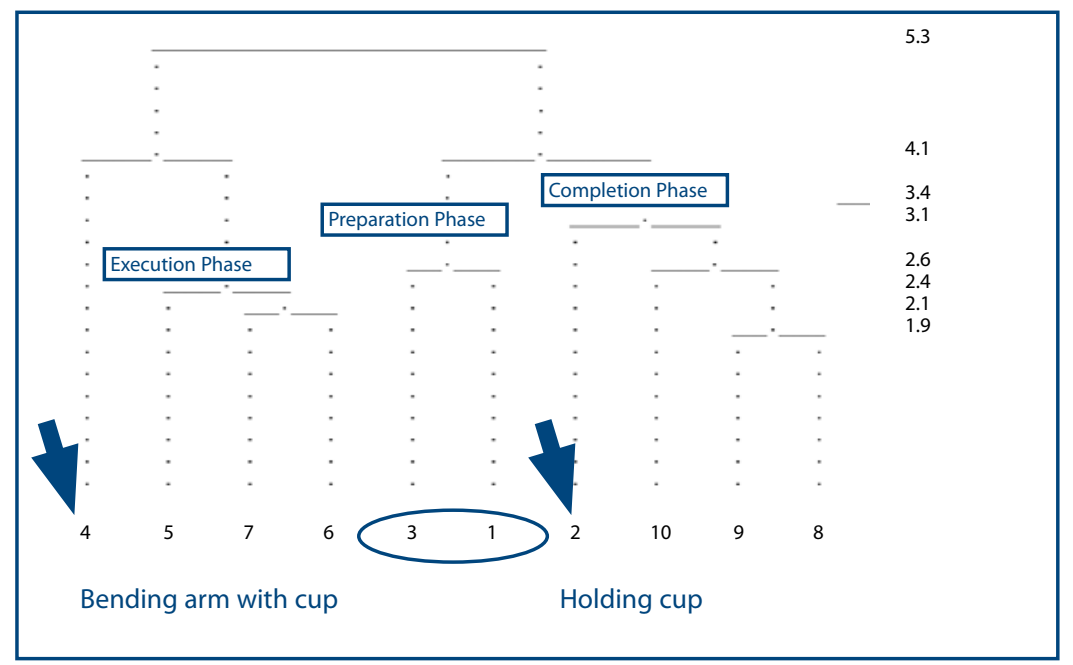

Fig. 2: Tree diagram of an stroke patient with minor problems performing the task. Two aspects of figure two are of main interest. First, the second action cluster 'grasping the cup' is clustered in the completion phase instead of the preparation phase. Second, the fourth action cluster, 'bending the arm with the cup' is isolated relatively early in the movement organization. Although it is embedded in the execution phase, its relation to the other actions in that phase is not very strong.

\section{Patients with 'abnormal' tree diagrams}

The tree diagrams of the second subgroup $(n=12)$ within the stroke population did not resemble the tree diagrams of the subjects within the control group, and there were no common characteristics between the 12 patients. There was a limited functional relation between actions and larger number of separate parts of the movement, judged as standalone units by the patient, resulting in:

- blurred, or undetectable phases;

- weak relations;

- many standalone, unintegrated actions.

Therefore, a few individual results will be presented below to illustrate our findings. Informally we noted that the abnormalities seen in the tree diagrams were similar to the abnormalities in motor performance during drinking out of a cup observed by the therapist (and partly reported by the patient). The group is otherwise clinically heterogeneous (tab. 3), but we did have one patient with aphasia who was able to understand and perform the test. Figure three shows a tree diagram of a patient with a non-functional dominant arm. Although the patient understood instructions for SDA-M assessment perfectly well, the only distinguishable phase was the completion of the task (numbers 8, 9 and 10, fig. 3, p 50). 


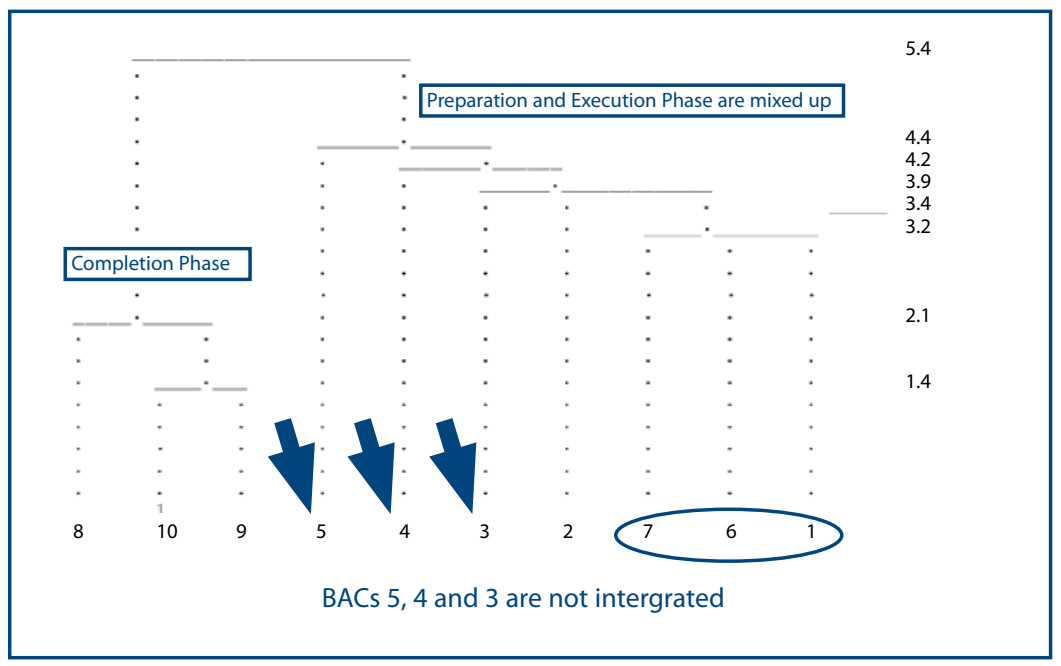

Fig. 3: Example of a tree diagram of a stroke patient with a non-functional dominant arm. The preparation and execution phase are brought together as one and several actions are not integrated but seen as separate equally unimportant parts of the movement (numbers 3,4 and 5). The only cluster structure in this part of the movement involves the numbers 1, 6 and 7. 'Reaching for the cup' (number 1) is related to the actions 'tilting the cup' and 'closing the lips and swallowing'. The patient was not able to perform this task as an overt movement in therapy.

A tree diagram from a patient with motor and more explicit cognitive problems is given in figure four. No logical clustering structure is recognizable and all actions seem equally (un)important to the patient.

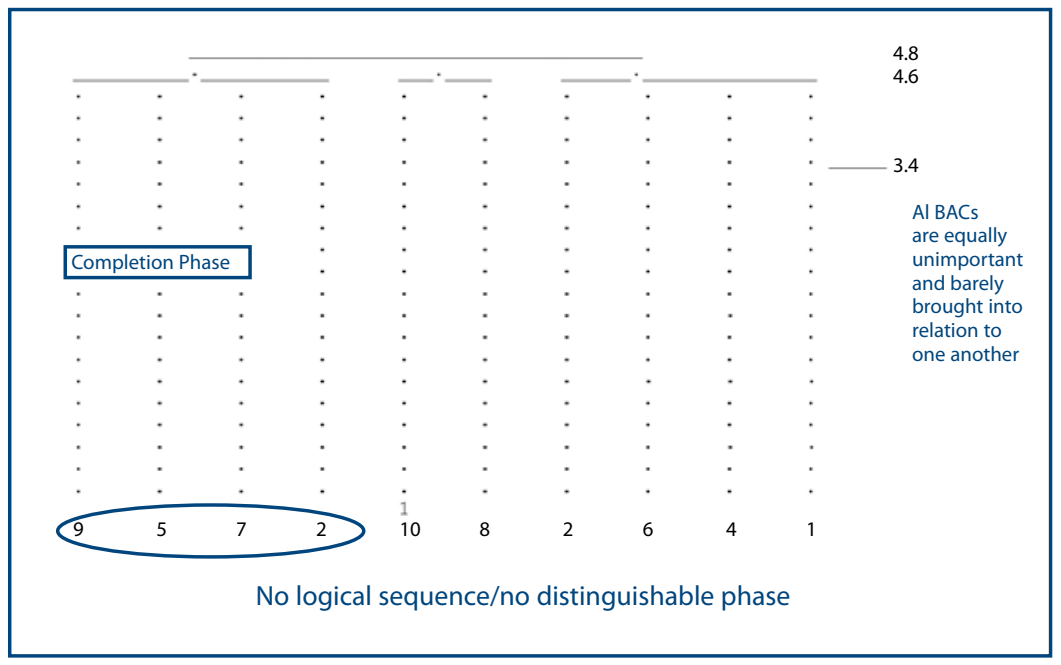

Fig. 4: Example of a treediagram of a stroke patient with physical and cognitive problems. 


\section{Discussion}

This initial study of the Structural Dimension Analysis of Motor memory (SDA-M) showed that it was possible to create a standard instruction protocol and procedure for the SDA-M for use with healthy elderly subjects and patients following stroke in order to visualize the actions needed when 'drinking out of a cup'. The technique was simplified compared with its form in sport (16). Second, within the group of neurological healthy people, the results were reproducible and the tree diagrams from the individuals within this group resembled each other. This would suggest that the SDA-M program was able to reproduce results in subjects in which the representation of a movement can be considered stable. Third, we found that in the stroke population, the representations could be visualized through the splitting procedure as well, and patients appeared to fall into two groups. The first, smaller population in this study, had tree diagrams similar to those seen in healthy controls with movements represented in logical and meaningful phases. The larger group showed representations that differed greatly from those seen in the control group.

The only clinical characteristic that related to tree diagram abnormalities was the side of the lesion: patients with right hemisphere lesions showed less stable clusters compared with patients with left hemisphere lesions. These differences have not yet been related to more specific localization, to specific impairments, or to size of the lesion, so that possible influences of altered visuospatial and motor attentional networks on SDA-M outcomes could not be determined.

One concern is that subjects may have misunderstood what is required, or misinterpreted some of the photographs, for example being uncertain whether a photograph concerned moving the arm away from or towards the mouth (numbers 4 and 8 ). The technique itself could be improved further. However, the control group seemed to be consistent, suggesting that the technique is certainly potentially usable and stable given reasonable circumstances.

At present, we cannot know that the results necessarily bear any relationship to internal processes that initiate and control complex goal-directed motor movements. Nonetheless, the consistency of response, certainly in the healthy population would suggest that this technique is collecting data on stable mental representation.

The apparent sensibility of the identified clusters would suggest that these representations are related to the overall task. And the evaluative studies in sport would suggest that changing these representations can alter performance. 
Consequently it seems plausible that the SDA-M is indeed measuring a functionally important phenomenon.

This study suggests that the SDA-M gives potential additional information, which might both detect motor planning faults and increase feedback efficiency. The potential clinical strength of the SDA-M lies in interpreting the tree diagrams, in combination with observations of the overt movement and information given by the patient, in order to:

- understand how motor action plans are disrupted by neurological damage in individual patients;

- use this information to plan specific mental $(5,22,23)$ and/or physical treatments.

Further conclusions cannot be drawn. The sample was relatively small and selected. Some of the similarities and differences found were statistically significant and not surprising, but should not be accepted without further study.

Future work should improve the technique for use in people with neurological disease, and should develop different activities for investigation. Then the technique should be investigated both as a diagnostic tool (identifying disordered central representations) and as a therapeutic tool (suggesting specific treatments) $(1,24)$. In the meantime, we believe that this is potentially an important tool both therapeutically, and in gaining a better understanding of the function and organization of the brain.

\section{Clinical messages}

- The Structural Dimension Analysis of Motor memory (SDA-M) can be used with stroke patients.

- The results show differences with healthy controls, suggesting it is a valid measure of motor action plans.

- The results are similar in one week.

\section{Acknowledgements}

We would like to thank the staff, inhabitants and the participating family members of the Klevarie nursing home, as well as the inhabitants of residence Sonneville, Maastricht, the Netherlands, participating in this study. We would also like to express our gratitude to Frank Roberts, Johan Ritzen and Danny Trines of the Proeftuin, Zuyd University of applied sciences in Heerlen, the Netherlands for adjusting the SDA-M program and Tom Heinen, $\mathrm{PhD}$, of the Psychological Institute of the Sports University of Cologne, Germany, for his support on the statistical analysis. 


\section{References}

1. Braun SM, Beurskens AJ, Borm PJ, Schack T, Wade DT. The effects of mental practice in stroke rehabilitation: a systematic review. Arch Phys Med Rehabil 2006;87:842-52.

2. Schack T, Tenenbaum G. Perceptual and cognitive control in action - a preface. New perspectives in movement science. Special issue part I: perceptual and cognitive control. Int J Sport Exerc Psychol 2004;2:207-209.

3. Schack T. the cognitive architecture of movement - New perspective in movement science. Special issue part II: representation and planning. Int J Sport Exerc Psychol 2004;2:403-38.

4. Decety J, Grezes J. Neural mechanisms subserving the perception of human actions. Trends Cogn Sci 1999;3:172-78.

5. Decety J, Jeannerod M, Durozard D, Baverel G. Central activation of autonomic effectors during mental simulation of motor actions in man. J Physiol 1993;461:549-63.

6. Decety J, Perani D, Jeannerod M et al. Mapping motor representations with positron emission tomography. Nature 1994;371:600-02.

7. Ingvar DH, Philipson L. Distribution of cerebral blood flow in the dominant hemisphere during motor ideation and motor performance. Ann Neurol 1977;2:230-37.

8. Mellit E, Petit L. Reopening the mental imagery debate: lessons from functional anatomy. Neuroimage 1998;8:129-39.

9. Nair DG, Purcott KL, Fuchs A, Steinberg F, Kelso JA. Cortical and cerebellar activity of the human brain during imagined and executed unimanual and bimanual action sequences: a functional MRI study. Brain Res Cogn Brain Res 2003;15:250-60.

10. Rizzolatti G, Fadiga L, Matelli M et al. Localization of grasp representations in humans by PET: 1. Observation versus execution. Exp Brain Res 1996;111:246-52.

11. Jeannerod M. Mental imagery in the motor cortex. Neuropsychologia 1995;33:1419-32.

12. Jeannerod M. Action from within. Special issue: New perspective in movement science, Part II: representation and planning. Int J Sport Exerc Psychol 2004;2:376-402.

13. Koch I, Keller P, Prinz W. The ideomotor approach to action control: implications for skilled performance. Special issue: New perspective in movement science, part II: representation and planning. Int J Sport Exerc Psychol 2004;2:362-75.

14. Schack T. On the structure of movement representations - Theoretical assumptions and methodical approach. Motor Control Learn 2001: 1-12. (E-journal: ISSN 1439-7919). Accessed 1 May 2004 from: http:/ /journal.motorik.swi.uni-saarland.de/index.htm.

15. Schack T, Mechsner F. Representation of motor skills in human long-term memory. Neurosci Lett 2006;391:77-81. 
16. Schack $\mathrm{T}$. The relationship between motor representation and biomechanical parameters in complex movements. Towards an integrative perspective of movement science. Eur J Sport Sci 2003;3:1-13.

17. Schols JM, Crebolder HF, van Weel C. Nursing home and nursing home physician: the Dutch experience. J Am Med Dir Assoc 2004;5:207-12.

18. Gould D, Damarjian N, Greenleaf C. Imagery training for peak performance. In: Raalte van J, Brewer WB, editors. Exploring sport and exercise psychology. Washington (DC): Am Psychol Assoc; 2002. p 49-74.

19. Malouin F, Richards CL, Doyon J, Desrosiers J, Belleville S. Training mobility tasks after stroke with combined mental and physical practice: a feasibility study. Neurorehabil Neural Repair 2004;18:66-75.

20. Martin K, Moritz S, Hall C. Imagery use in sports: a literature review and applied model. Sports Psychol 1999;13:245-68.

21. Lander HJ, Lange K. Eine differentialpsychologische Analyse begrifflich-strukturiertes Wissens. Z Psychol 1992;200:181-97.

22. Decety J. The neurophysiological basis of motor imagery. Behav Brain Res 1996;77:45-52.

23. Jackson PL LM, Malouin F, Richards CL, Doyon J. Functional cerebral reorganization following motor sequence learning through mental practice with motor imagery. Neuroimage 2003;20:1171-80.

24. Jackson PL, Lafleur MF, Malouin F, Richards C, Doyon J. Potential role of mental practice using motor imagery in neurologic rehabilitation. Arch Phys Med Rehabil 2001;82:1133-41. 




\section{Chapter four}

Using mental practice in stroke rehabilitation: a framework

Braun SM, Kleynen M, Schols JM, Schack T, Beurskens AJ, Wade DT.

Clin Rehabil 2008;22:579-591 


\section{Abstract}

Introduction: Motor imagery and mental practice are getting increased attention in neurological rehabilitation. Several different mental practice intervention protocols have been used in studies on its effect on recovery in stroke rehabilitation. The content of the intervention protocols itself is rarely discussed or questioned.

Objective: To give a practical framework of how mental practice could be integrated into therapy, drawing on available evidence and theory. The aim of the treatment program described is to enhance both the patient's physical performance and their empowerment and self-deter-mination.

The framework: Based on evidence from sports rehabilitation and our own experiences the framework will eventually be evaluated in a randomised controlled trial. Five steps are described to teach and upgrade the patient's imagery technique: (1) assess mental capacity to learn imagery technique; (2) establish the nature of mental practice; (3) teach imagery technique; (4) embed and monitor imagery technique; (5) develop self-generated treatments. The description is not, however, a recipe that should be followed precisely. It leaves enough room to tailor the mental practice intervention to the specific individual possibilities, skills and needs of the patient in accordance with evidence based practice.

Discussion: Different aspects of the described protocol are discussed and compared to experiences from sports and evidence available in rehabilitation. 


\section{Using mental practice in stroke rehabilitation: a framework}

\section{Introduction}

Imagery used by sportsmen may be applicable in rehabilitation $(1,2)$, and indeed imagery techniques have been tried as treatments in neurological population. The basic idea is 'imagining oneself undertaking the skilled movement without actually doing the movement'. The subject may imagine the movement in the third (as if looking from a distance at oneself) or the first (as if looking through their own eyes) person's view (3, 4). 'Mental practice' describes a training or therapy form in which an internal representation of the movement is activated and the execution of the movement repeatedly mentally simulated, without physical activity, within a chosen context. It is used for the improvement or stabilization of a given set of movements $(1,5)$.

There is controversy about the imagery subject and many different intervention forms are used in both research and daily care. In this paper, we do not want to compare, evaluate or value different imagery techniques. We do wish to discuss how imagery might be used in practice. Even though we are still in the process of evaluating the effects of this concept in a randomised trial (6), we believe it important to describe and discuss the content because (a) many therapists wish to use mental practice already and (b) it might help standardize the interventions subjected to evaluation, or at least encourage accurate description of other methods.

This article concentrates on the specific features of using mental practice: general aspects of rehabilitation are not described.

\section{The three pillars}

Three pillars are involved in developing an imagery framework in real care: the patient, the evidence and the therapist. When we started building a theoretical framework for imagery in stroke rehabilitation we wanted to come as close as possible to the situation in real care. Three aspects are generally considered by therapists when making therapy decisions: the patient's abilities and preferences for therapy, the evidence existing for a specific intervention (efficacy and value) and the therapist's abilities and preferences for therapy $(7,8)$.

\section{I) The patient}

The mental practice intervention as we have used is patient orientated. Patients are able to choose the meaningful activities they want to improve. The mental practice intervention is then adjustable not only to the practiced motor action but also to the individual patient's abilities. As the imagery technique can be used outside regular therapy time and preferably in the patient's own domestic environment, it is important that it is taught correctly and incorporated 
into daily activities. Only then will patients achieve increased empowerment by the use of imagery. Patients are given tools to decide for themselves how and when their rehabilitation process takes place. Moreover, this increase in the feeling of autonomy, of independence, may be another as yet unexplored reason for considering imagery as an additional rehabilitation technique. Tailoring interventions and giving autonomy to the patient in their rehabilitation process are important aspects of client centred care.

\section{II) The evidence}

The mental practice intervention we use and describe here includes both available evidence from sport science and rehabilitation, and our own experience and research (1, 2, 9-17).

Results from brain imaging techniques have shown that mental practice may alter neural function (18-26). The basic idea of using thought to improve motor function is founded on this research. Also, there is some evidence that at least some patients with stroke have intact imagery skills $(22,27)$.

Evidence from sports is reasonable even though studies from different sports are hard to compare. However, various aspects of the content of an effective mental practice intervention can be derived from studies in sports science. Several applied models can be transferred to rehabilitation, as the steps to follow are not essentially different for athletes and patients. In addition, guidelines for teaching and developing imagery can be abstracted from sports. For instance, the need to combine overt movement with imagery to enhance the vividness or the necessity to teach imagery before using it are principles transferred from sport to rehabilitation $(28,29)$.

Evidence from rehabilitation is promising but still limited. Most studies have described their intervention weakly, if at all (30-35). Where sports literature clearly divides imagery techniques and uses applied models to categorize different aspects of mental practice, the descriptions available in rehabilitation suggest a huge range of actions that all have been included under the rubric of 'mental practice', which makes meta-analysis or comparison of results difficult.

General principles from rehabilitation guidelines and proven aspects of stroke therapy were incorporated as much as possible in the mental practice framework. For instance, the evidence that more practice is more effective $(13,14,36)$. Mental practice can be performed by the patient him- or herself without therapists being present, which seems to be an addition to existing therapy. Training intensity can be increased substantially. Another example is that motor tasks should be practiced in the patient's context (16) to be most effective. 


\section{III) The therapist}

Many rehabilitation professionals report that they already use imagery in their work. They simply ask patients to imagine movements, but they do not know how to teach patients the use of movement imagery and to monitor outcome. Here we describe only an outline, so that the intervention becomes dynamic: the professional can use his or her experience and expertise to tailor the mental practice content to the abilities and preferences of the patient and the context in which is practiced.

\section{Mental practice in rehabilitation: an overview}

The framework is divided into five steps (fig. 1, tab. 1, p 63). The first step is for the therapist to decide using clinical judgment whether the patient is a suitable candidate for mental practice by assessing the mental capacity of the patient to participate.

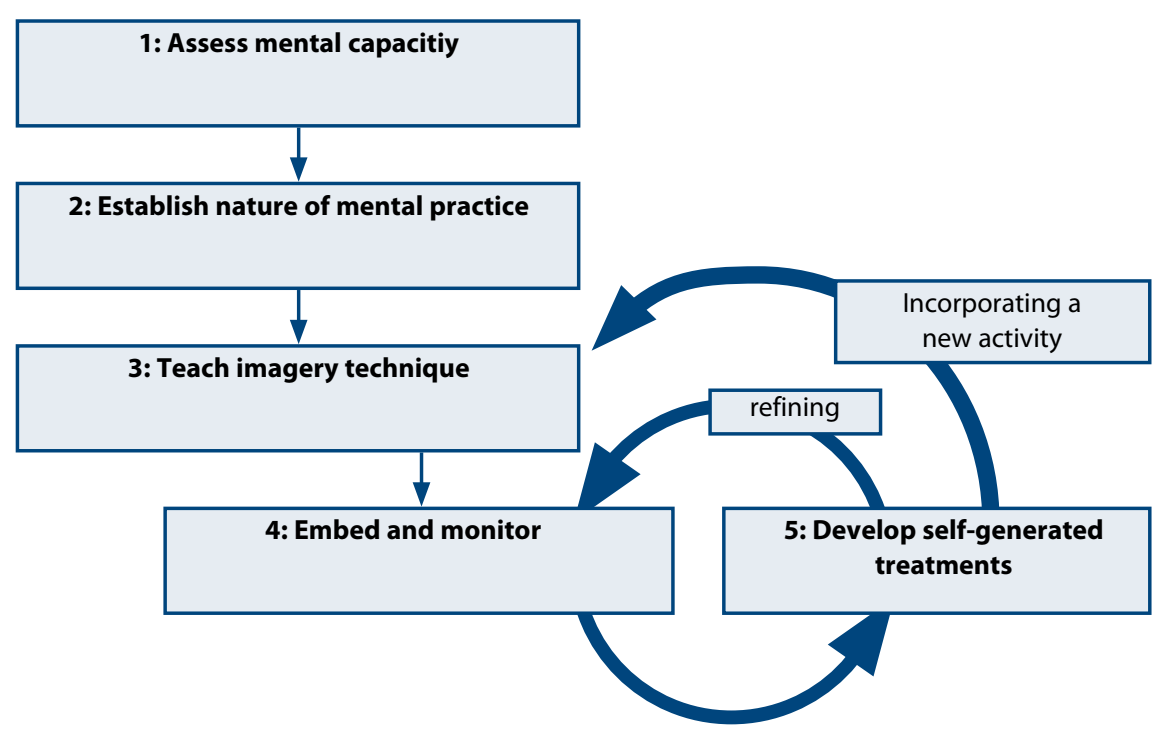

Fig. 1: Overview of the steps taken in the mental practice intervention protocol. 
The next step is to establish with the patient the nature of the treatment: what is it, and how might it help. An important aspect is to relate imagery to the patient's own experiences so he or she grasps the idea of imagery and how it might help recovery.

Then, the skill of mental practice should be taught. In this part of the protocol, the imagery technique is optimized, accepting the constraints of the individual patients but improving the technique where possible. This step takes place during therapy, closely monitored by the therapist.

Once the patient has some basic ability in imagery, it is embedded in other active treatments and, as soon as possible, in unsupervised practice.

Throughout, the therapist needs to be aware that change in performance may require changes in the imagery - the specific tasks practiced will need upgrading as recovery of motor functions occurs, and the general technique may also need to be improved.

Finally, the patient should learn how to develop self-generated treatments. Therapist support should be reduced gradually and the patient discharged still motivated to use and develop mental practice, always able to make contact again if problems arise.

In this article, we use patient and therapist examples from our studies at the Klevarie nursing home (KNH) in Maastricht, Holland, the Oxford Centre for Enablement (OCE) in Oxford, England and the BioMetrical Centre (BMC) in Gulpen, Holland, to illustrate the several steps. The quotations were taken from semi-structured interviews during research projects with these stroke patients and their therapists.

\section{Assess the mental capacity of your patient (clinical judgment)}

Before teaching a mental practice protocol, you should check whether your patient has sufficient mental capacity to learn the imagery techniques (as one would check before any other rehabilitation treatment). You should establish whether your patient has enough attention, working memory, perception and motivation to follow imagery instructions (37). Not every athlete uses imagery techniques and it is very probable that not every patient is able or wants to perform mental practice. We have found no evidence for specific tests with a prognostic value for the use of imagery. We suggest that a patient should be able to remember and follow a three-stage command. In addition, the patient should be able to participate actively in other rehabilitation activities for at least 10 minutes. 


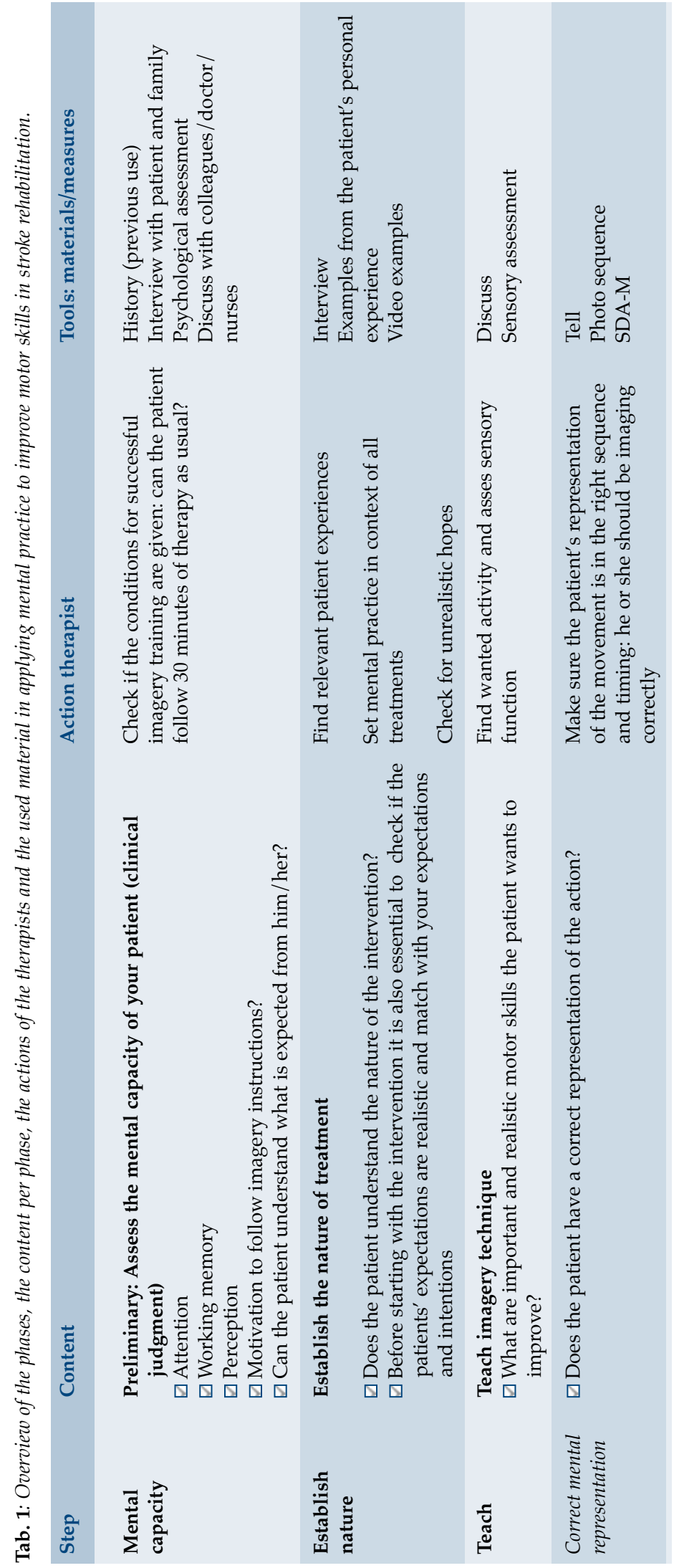




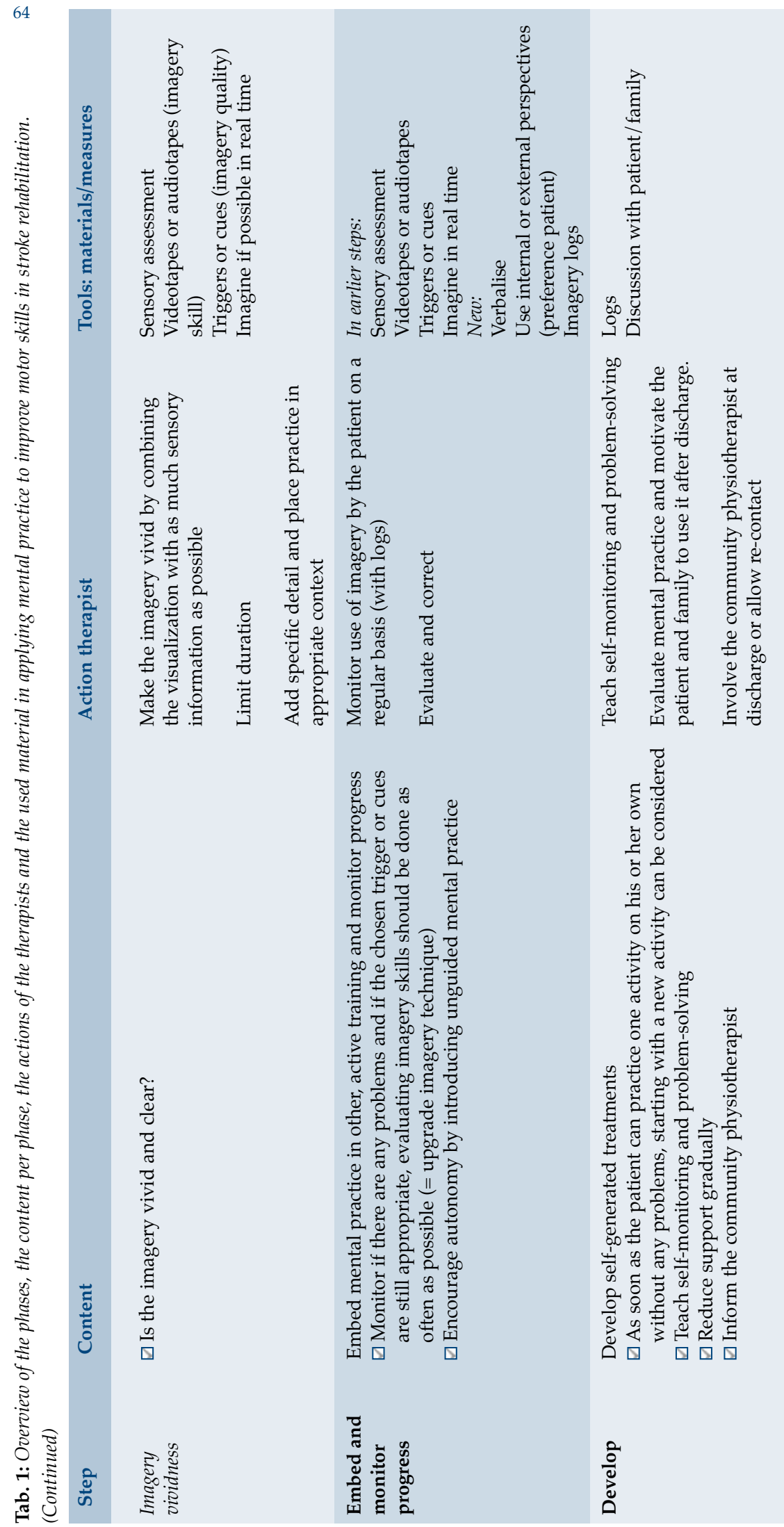




\section{Establish the nature of treatment}

Engaging the patient in understanding what imagery is and making a connection between the patient's experience and the imagery technique may be essential for the patient to grasp the idea completely. When mental practice is explained, familiar examples should be used. The most common examples come from sports, but it is our experience that some elder stroke patients have no interest in sports. You need to find relevant patient experiences to work with.

'I had never heard of imagery or movement imagery as a training method before. My therapist asked me about my hobbies and I told her I used to play in a brass band. She asked if I ever used to practice in my mind and then I remembered what our conductor used to say. 'If you wanted to place your lips correctly on the trumpet, you should imagine saying the letter ' $p$ '. This helped me create the right sound. I decided that I should give imagery a try'. Patient (BMC 2007)

When you explain imagery, you may want to stay close to real-life experiences of your patients. What did they do for a living? What hobbies did they have? If they can recall sensory sensations from these activities, you are more likely to motivate and explain what you want your patient to do during imagery.

It is also important that patients are aware that mental practice is a complementary addition to physical exercise and that it may help reach higher levels of performance: they should not see it as a single, isolated treatment. Mental practice should be set in the context of all treatments, and therapists should stress the need to practice imagery on a regular basis (11).

Finally, unrealistic expectations of patients should be addressed immediately. For example, any belief that 'I only need to imagine movements and eventually I will succeed' should be removed. Interviewing the patient on his beliefs and expectations is important in all rehabilitation, but especially when an unusual treatment is being introduced. Therefore, before proceeding, check that the patient understands what imagery is and how it is part of the rehabilitation process. Following this it is obviously also important to choose a task that the person wants to improve at, that is motivating and that is potentially achievable - this is part of goal setting in all rehabilitation. 


\section{Teaching imagery technique}

Before mental practice can be embedded in and outside of therapy (unguided), it is necessary to optimize the imagery technique to the patient's abilities (38).

'One of my patients chose to practice the activity 'drinking from a cup' with the affected arm. He could describe perfectly well all (relevant) steps of the movement in the correct sequence. However, the patient told me that he was only able to 'see' himself. It was not possible to generate other sensory input during imagery. To improve the vividness we started to combine imagery of the movement with the real performance. During and after the overt movement, I systematically asked the patient what he was feeling, hearing, smelling and tasting. For example: How does it feel to grasp the cup? Is the cup warm or cold? How does the structure of the cup feel? With these questions I tried to focus him on those sensory details'. Therapist (KNH 2006)

Two components of any task to be rehearsed mentally are important: the component actions of the task and having vivid images.

It is likely that the underlying pathology will disrupt neurological mechanisms that plan and control the movements necessary for a task. Patients may not have a clear representation of the actions needed and used to build their imagery on.

Therefore, the therapist should establish whether the patient knows the sequence and timing of the actions needed to achieve the task successfully. The simplest way is asking the patient to describe a movement sequence.

From the example below, several parts of the movement can be distinguished (lifting the cup, tilting it etc). The description covers all the actions and phases of the movement and leaves no hiatus in the movement sequence. Consequently, this patient knows theoretically how to perform 'drinking from a cup'.

'When I imagine myself drinking coffee, I need to remember several things. First, I have to sit upright in front of the table. My hands are lying on my lab. Before grasping the cup, I must lift my hand and straighten my arm. I am very careful not to move too quickly: otherwise it is too difficult to control my hand. When my fingers reach the cup, I open my hand to grasp the cup at the base. I lift the cup being careful to keep it in balance. My elbow bends and the brim of the cup touches my lip. I have to tilt the cup, very carefully again because I do not want to spill. When I feel the coffee on my tongue and close my mouth. To swallow I lean my head a little bit back. The next step is to tilt the cup back and straighten my elbow. Slowly I put the cup down. As soon as the cup is standing on the table again, I open my hand again and let go of the cup'. A patient describing the task 'drinking from a cup'. $(\mathrm{KNH}, 2006)$ 
Putting pictures of a movement in the right order is another way of getting an idea of how a patient structures a movement. In figure two part of the motor sequence from drinking from a cup is shown. This method is especially helpful when patients have aphasia or other communication difficulties: this is important because such patients are also more likely to have a disrupted motor plan from apraxia.
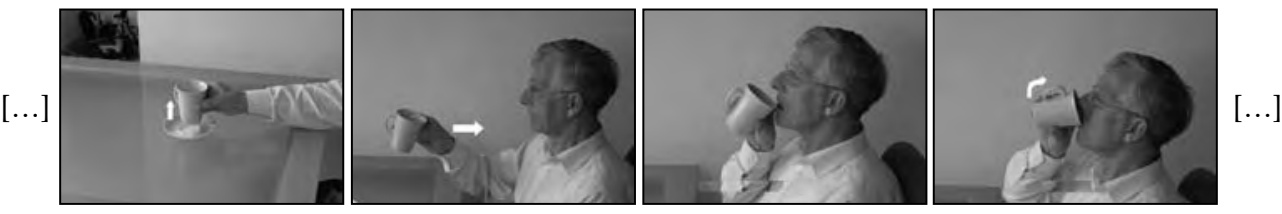

Fig. 2: Part of the motor sequence used in drinking from a cup.

Another option is a computer program called the Structural Dimension Analysis of Motor memory' (SDA-M) (39). We use the program to judge the mental representation of a motor action. The result of a cluster analysis with the SDA-M is a tree diagram from which several aspects can be judged (40):

- Is the clustering of the actions meaningful? In other words, can the movement organization lead to a successful performance?

- Are all actions integrated in the movement or a phase of the movement?

- Which actions relate strongly to each other?

- Are any of the actions related to a part of the movement in which they do not belong?

Moreover, does this affect the success of the movement performance?

If the mental representation is not correct or incomplete, rehearsing the sequence by verbalizing the steps or putting pictures of the steps in a right order can be used to improve the representation. Verbalizing of the movement parts can be done during or after observation or performance of the task and should be corrected or completed by the therapist. This should be repeated until the correct sequence is stabilized.

Once you are assured that your patient has a correct mental representation of the motor action you want to improve, you can start making the image livelier.

First, the patient should be consciously aware of what he or she feels, sees and hears during movements. Combining imagery with overt movement makes it more likely that they will mentally recreate these experiences during and through imagery. It is essential to find out which triggers or cues can be used to facilitate the quality of the imagery. You can also get 
useful information about the vividness of the imagery by discussing it with the patient. By systematically asking the patient to recollect the sensations, you can try to enhance the vividness of the imagery. It is also important to recognize that impairment arising from the disease may limit some sensory inputs and sensory images.

It is our experience that patients cannot focus on a movement during imagery for longer than half a minute. This is similar to the time injured athletes spend on imagery (41) and is probably true of most people. Consequently, one should limit the duration of imagery rehearsals to the patient's ability and practice in short spells $(28,29)$. It seems better to have a few good images than many blurry ones.

\section{Embed mental practice in other active training, and monitor progress}

Once the therapist is confident that a patient is able to perform mental practice the actual training phase starts. As recovery occurs, the sensory input of the overt movement may change over time and so imagery and overt movement should be combined on a regular basis, in order to link the kinesthetic feelings caused by the improved overt movement to the imagined feeling. The therapist can help upgrade the technique and thus improve the imagery technique. Monitor if there are any problems and if the chosen trigger or cues are still appropriate, evaluating imagery skills should be done on a regular basis.

'Soon I noticed that the best times for me to practice were the early morning when everybody else was still asleep and at nights lying in bed. Normally I practiced 5 to 10 minutes. I made it habit to first practice my walking mentally before actually walking on the corridor or in the gym. I started looking more closely at the gait of other patients, trying to see what they did better or less well'. Patient (OCE, 2007)

Our patients have generally used different mental practice strategies. If a patient has difficulties with a specific part of a movement, such as putting the cup on the table without spilling or tipping it, he simply 'practices' this part mentally and embeds it in the entire movement later.

Other patients may practice the entire movement from the start. Most use the actual sensory input of a movement during imagery. However, a small group of patients remember how a movement used to feel before their stroke - effortless, fluent and strong. The latter group uses these sensations and visualization during imagery instead. From our observations, these patients are either younger and/or the stroke occurred recently. 
As soon as possible, the patient should be encouraged to use mental practice outside therapy. The unguided mental practice not only increases therapy intensity but can also give the patient a feeling of empowerment.

To get an idea what patients are doing during unguided imagery, they can be asked to use a log in which they can record how long, how often and how well practice on a daily basis went.

'After about two weeks of practising my gait mentally, my therapist and I decided that my imagery skills were good enough to start practising on my own. He told me that I should try to practice as often as possible and as long as I liked, but I needed to record my imagery efforts in a logbook. On a daily basis I wrote down the time spend on imagery, which tasks I had practiced, how I felt that day and whether or not it went well. I liked being partly independent from my therapist and therapy times'. Patient $(\mathrm{KNH}, 2006)$

In our log (appendix) patients also rate how they felt during that day by underlining the appropriate smiley face because mood and fluctuation in motivation may influence the imagery. If a patient is having a bad day or is not feeling well, there is a good chance that he or she will practice less or not as well as usual. The bottom open space leaves room to add any comment they feel important with regard to the mental practice. At the beginning of a therapy session, the $\log$ can be used to discuss progress and difficulties of unguided mental practice.

\section{Develop self-generated treatments}

As soon as the patient can practice one activity in combination with mental practice on his or her own without any problems, a new activity can be considered. Interestingly enough, some patients report that they do use imagery for one specific movement but are unable to use it for another ('I do not know why the movement sometimes goes wrong, and why it goes right, so I do not actually know what I should imagine', Patient (KNH, 2006)). It seems important to teach patients how to self monitor their imagery technique and solve problems along the way. This could increase the chance that the technique can be generalized to other motor actions (32).

'Most patients value the feeling of autonomy they get from using imagery. Being able to practice safely, independently of any care giver, gives empowerment to the patient. We sit together and discuss some activities, which could be considered for practice: for instance the correct use of a rollator at home. Together we discuss which problems there might occur and how mental practice could be used to tackle those problems'. Therapist (OCE, 2007) 
Having established a certain amount of autonomy, the therapist should reduce support gradually. This way the patient learns to depend on his or her own judgment and can estimate his/ her problem solving skills.

At discharge, you can evaluate with the patient and his or her family how the imagery skills have developed and if the patient is motivated and confident he or she will use it at home. Expected obstacles and motivational problems could be discussed and ways of gaining further advice outlined, such as involving a community physiotherapist or allowing further contact. This last step is not obtainable for every patient. There is little evidence that learned task can be generalized to other situations or other tasks, but there is some evidence that a problem solving strategy could (32).

\section{Duration of an imagery program}

How long each step takes depends on the abilities of the patient to learn and use motor imagery. Our experience is that the first three parts could be completed in two weeks time (three to six sessions). Having established a correct imagery technique, unguided training may be added to the guided in order to increase therapy time safely. This training can extend over a period of weeks or months, depending on success rate. Before discharge from the rehabilitation centre or nursing home, some time should to be reserved for evaluating and adjusting the imagery program (tab. 1).

\section{Discussion}

This article has described a theoretical and practical framework for using imagery in rehabilitation with examples from nursing homes and rehabilitation centres. It is not restricted, however, to the use within these settings. It is also possible to use this framework for instance as a community therapist at a patient's home. Moreover, it can be used by any rehabilitation professional.

Some notes on the limitations of mental practice seem appropriate. Although mental practice may have benefits, it is probably not feasible for all patients due to the effect of stroke and the preferences of patients. It is not a cure for everything, but an addition to the hard physical work that still needs to be done by the patient.

The main aim of describing the intervention in detail is to contribute to discussion about how imagery could be taught in rehabilitation. Using applied models, from sports like Martin et al (15), might help reduce the variation of mental practice goals and content in rehabilitation. An alternative is answering the four w's of imagery questions. 
The four w's of imagery in sports

In 2000, Munroe and co-workers introduced the four questions concerning imagery use in athletes: When do athletes use imagery? Where do athletes use imagery? Why do athletes use imagery? What do athletes image?

Imagery is used by athletes in competition, training and rehabilitation ('when'). The use of imagery during injury is perhaps the most close to the use of imagery by patients in rehabilitation. Driediger and co-workers performed a qualitative research to gain more insight on the remaining three questions by interviewing 10 injured athletes from seven different sports on their imagery habits (41).

Athletes practiced most at actual practice sessions (when not participating), at home in bed and while driving ('where').

Athletes reported eight reasons (functions) why they use imagery. For example to rehearse specific movements or rehabilitation exercise, to increase motivation, reduce anxiety, stimulate healing, pain management, and injury prevention.

Finally, 'what' athletes imaged depended largely on the content of the imagery. Interestingly, within the small group of interviewed athletes a large range of answers with regard to the frequency and duration of the imagery sessions was given. Imagery time ranged for instance from five to 30 seconds. Athletes reported the use of visual, auditory, kinesthetic, imagery of past events and imagery of athletes' health from an internal and external view. It seems as if the personal preferences play an important role in developing an individual imagery program. Most athletes believed imagery to be effective, specifically during rehabilitation.

\section{The four w's of imagery in rehabilitation}

The 'where' question in rehabilitation can be answered by any place therapy takes place. This can be at home, a rehabilitation ward or at a community therapist's practice. However in rehabilitation a second 'where' relates to the imagined location, and this should be a place familiar to the patient, usually home for most activities.

With regard to the 'when' question, our experiences are that patients spend more time observing other patients during and outside of therapy, analysing motor actions and repeating them cognitively. 'In bed' and 'while waiting for therapy' are also reported by patients. 
In rehabilitation, imagery is mainly used ('why') for improving skills and motor functions. However, the use of other imagery types to change cognition, limit anxiety and arousal might just be as effective as or more effective for recovery than improving motor actions with mental practice. The use of imagery for pain management, increasing confidence or maintain a positive attitude and healing may be an interesting addition to the cognitive specific function $(2,15,43,44)$.

Patients often report an increased feeling of autonomy by using imagery as an addition to physical therapy. Unguided imagery training in particular makes them independent from guided therapy time. Being able to practice whenever and wherever the patient wants motivates most patients to work even harder at recovery.

Little is known about 'what' patients visualize and feel during imagery. The vividness of the imagery will most probably be limited due to the effects of the stroke. Most likely, patients will have different preferences in developing imagery skills as well.

\section{Clinical messages}

- Use clinical judgement to determine whether your patient is a suitable candidate for mental practice.

- Teach imagery of correct motor representations, make it vivid and then use it on a regular basis.

- Motivate the patient to use imagery unguided.

- Develop self-generated treatment and make support available after discharge.

\section{Acknowledgements}

We would like to thank the paramedical staff of the Klevarie nursing home for the constructive discussion and critical notes on the mental practice intervention content.

\section{References}

1. Braun SM, Beurskens AJ, Borm PJ, Schack T, Wade DT. The effects of mental practice in stroke rehabilitation: a systematic review. Arch Phys Med Rehabil 2006;87:842-52.

2. Dickstein R, Deutsch JE. Motor imagery in physical therapist practice. Phys Ther 2007;87: 942-53.

3. Jackson PL, Lafleur MF, Malouin F, Richards C, Doyon J. Potential role of mental practice using motor imagery in neurologic rehabilitation. Arch Phys Med Rehabil 2001;82:1133-41. 
4. Sharma N, Pomeroy VM, Baron JC. Motor imagery: a backdoor to the motor system after stroke? Stroke 2006;37:1941-52.

5. Schack T. Mentales Training. In: Strauss B, editor. Handbuch Sportpsychologie. Schorndorf: Hofmann; 2006. p 254-61.

6. Braun SM, Beurskens AJ, van Kroonenburgh SM, Demarteau J, Schols JM, Wade DT. Effects of mental practice embedded in daily therapy compared to therapy as usual in adult stroke patients in Dutch nursing homes: design of a randomized controlled trial. BMC Neurol 2007;7:34.

7. Sackett DL, Scott Richardson W, Rosenberg W, Haynes BB. Evidence-based medicine: how to practice and teach EBM. New York: Churchill Livingstone; 1997.

8. Sackett DL, Rosenberg WM, Gray JA, Haynes RB, Richardson WS. Evidence based medicine: what it is and what it isn't. BMJ 1996;312:71-2.

9. Commissie CVA-revalidatie. Revalidatie na een beroerte, richtlijnen en aanbevelingen voor zorgverleners. Den Haag: Nederlandse Hartstichting 2001.

10. de Vries S, Mulder T. Motor imagery and stroke rehabilitation: a critical discussion. J Rehabil Med 2007;39:5-13.

11. Gould D, Damarjian N, Greenleaf C. Imagery training for peak performance. In: Raalte van J, Brewer BW, editors. Exploring sport and exercise psychology. Washington (DC): Am psychol assoc; 2002. p 49-74.

12. Hall C. Imagery and the acquisition of motor skills. Canadian Journal of Sports Sciences 1992;17:19-27.

13. Kwakkel G, van Peppen R, Wagenaar RC, Wood Dauphinee S, Richards C, Ashburn A, et al. Effects of augmented exercise therapy time after stroke: a meta-analysis. Stroke 2004; 35:2529-39.

14. Kwakkel GK, BJ; Wagenaar, RC. Therapy impact on functional recovery in stroke rehabilitation: A critical review of the literature. Physiotherapie 1999;85:377-91.

15. Martin K, Moritz S, Hall C. Imagery use in sports: a literature review and applied model. The Sports Psychologist 1999;13:245-68.

16. Trombly CA, Wu CY. Effect of rehabilitation tasks on organization of movement after stroke. Am J Occup Ther 1999; 53: 333-44.

17. van Peppen RP, Kwakkel G, Harmeling-van der Wel BC, et al.: KNGF-richtlijn beroerte. Ned Tijdschr Fysiother 2004;114(Suppl):1-77.

18. Annett J. Imagery and motor processes. Br J Psychol 1995;86:161-7.

19. Decety J. The neurophysiological basis of motor imagery. Behav Brain Res 1996;77:45-52. 
20. Decety J, Grezes J, Costes N, Perani D, Jeannerod M, Procyk E, et al. Brain activity during observation of actions. Influence of action content and subject's strategy. Brain 1997;120:1763-77.

21. Jeannerod M. Mental imagery in the motor cortex. Neuropsychologia 1995;33:1419-32

22. Johnson SH, Sprehn G, Saykin AJ. Intact motor imagery in chronic upper limb hemiplegics: evidence for activity-independent action representations. J Cogn Neurosci 2002;14:841-52.

23. Malouin F, Richards CL, Jackson PL, Dumas F, Doyon J. Brain activations during motor imagery of locomotor-related tasks: a PET study. Hum Brain Mapp 2003;19:47-62.

24. Mellit E, Petit L. Reopening the mental imagery debate: Lessons from functional anatomy. Neuroimage 1998;8:129-39.

25. Weiss T. Activation Process During Mental Practice in Stroke Patients. International Journal of Psychophysiology 1994;17:91-100.

26. Yaguez L, Nagel D, Hoffman H, Canavan AG, Wist E, Homberg V. A mental route to motor learning: improving trajectorial kinematics through imagery training. Behav Brain Res 1998;90:95-106.

27. Johnson SH. Imagining the impossible: intact motor representations in hemiplegics. Neuroreport 2000;11:729-32.

28. Budney AJ, Murphy SM, Woolfolk RL. Imagery and Motor performance: What do we really know? In: Sheikh AA, Korn ER, editors. Imagery in Sports and Physical Performance. Farmingdale, NY: Baywood; 1994. p 97-120.

29. Driskell JE, Copper C, Moran A. Does mental practice enhance performance? J Appl Psychol 1994;79:481-92.

30. Crosbie JH, McDonough SM, Gilmore DH, Wiggam MI. The adjunctive role of mental practice in the rehabilitation of the upper limb after hemiplegic stroke: a pilot study. Clin Rehabil 2004;18:60-8.

31. Dijkerman HC, Letswaart M, Johnston M, MacWalter RS. Does motor imagery training improve hand function in chronic stroke patients? A pilot study. Clin Rehabil 2004;18:538-49.

32. Liu KP, Chan CC, Lee TM, Hui-Chan CW. Mental imagery for promoting relearning for people after stroke: a randomized controlled trial. Arch Phys Med Rehabil 2004;85:1403-8.

33. Malouin F, Richards CL, Doyon J, Desrosiers J, Belleville S. Training mobility tasks after stroke with combined mental and physical practice: a feasibility study. Neurorehabil Neural Repair 2004;18:66-75.

34. Page SJ, Levine P, Leonard A. Mental practice in chronic stroke: results of a randomized, placebo-controlled trial. Stroke 2007;38:1293-7.

35. Page SJ, Levine P, Leonard AC. Effects of mental practice on affected limb use and function in chronic stroke. Arch Phys Med Rehabil 2005;86:399-402. 
36. Kwakkel G, Wagenaar RC, Twisk JW, Lankhorst GJ, Koetsier JC. Intensity of leg and arm training after primary middle-cerebral-artery stroke: a randomised trial. Lancet 1999:17;354:191-6.

37. Malouin F, Belleville S, Richards CL, Desrosiers J, Doyon J. Working memory and mental practice outcomes after stroke. Arch Phys Med Rehabil 2004;85:177-83.

38. Wade DT. Personal context as a focus for rehabilitation. Clin Rehabil 2000; 14: 115-8.

39. Schack $\mathrm{T}$. The cognitive architecture of movement - New perspective in movement science. Special issue, part II: representation and planning. Int J Sport Exerc Psychol 2004;2:403-438.

40. Braun SM, Schack T, Marcellis RG, Oti KC, Schols J, Wade DT, Beurskens AJ. Is it possible to use the Structural Dimension Analysis of Motor memory (SDA-M) to investigate representations of motor actions in stroke patients? Clin Rehabil 2007;21:822-32.

41. Driediger M, Hall C, Callow N. Imagery use by injured athletes: a qualitative analysis. J Sports Sci 2006;24:261-71.

42. Munroe K, Giacobbi P, Hall C, Weinberg R. The four w's of imagery use: Where, when, why and what. The Sport Psychologist 2000;14:119-37.

43. Moseley GL. Graded motor imagery is effective for long-standing complex regional pain syndrome: a randomised controlled trial. Pain 2004;108:192-8.

44. Tamir R, Dickstein R, Huberman M. Integration of motor imagery and physical practice in group treatment applied to subjects with Parkinson's disease. Neurorehabil Neural Repair 2007;21:68-75. 


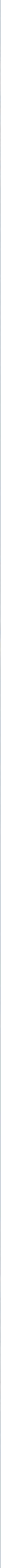




\section{Chapter five}

Effects of mental practice embedded in daily therapy compared to therapy as usual in adult stroke patients in Dutch nursing homes: design of a randomised controlled trial

Braun SM, Beurskens AJHM, van Kroonenburgh S, Demarteau J, Schols JM, Wade DT.

BMC Neurol 2007;7:34 


\section{Abstract}

Introduction: Mental practice as an additional cognitive therapy is getting increased attention in stroke rehabilitation. A systematic review shows some evidence that several techniques in which movements are rehearsed mentally might be effective but not enough to be certain. This trial investigates whether mental practice can contribute to a quicker and/or better recovery of stroke in two Dutch nursing homes. The objective is to investigate the therapeutic potential of mental practice embedded in daily therapy to improve individually chosen daily activities of adult stroke patients compared to therapy as usual. In addition, we will investigate prognostic variables and feasibility (process evaluation).

Methods: A randomised, controlled, observer masked prospective trial will be conducted with adult stroke patients in the (sub)acute phase of stroke recovery. Over a six weeks intervention period the control group will receive multi professional therapy as usual. Patients in the experimental group will be instructed how to perform mental practice, and will receive care as usual in which mental practice is embedded in physical, occupation and speech therapy sessions. Outcome will be assessed at six weeks and six months. The primary outcome measure is the patient-perceived effect on performance of daily activities as assessed by an 11 point Likert scale. Secondary outcomes are: Motricity Index, Nine Hole Peg Test, Barthel Index, Timed up and Go, 10 meters walking test, Rivermead Mobility Index. A sample size of the patients group and all therapists will be interviewed on their opinion of the experimental program to assess feasibility. All patients are asked to keep a log to determine unguided training intensity.

Discussion: Advantages and disadvantages of several aspects of the chosen design are discussed. 


\section{Effects of mental practice embedded in daily therapy compared to therapy as usual in adult stroke patients in Dutch nursing homes: design of a randomised controlled trial}

\section{Introduction}

Stroke is a major health problem, which is likely to increase due to aging (1-3). Patients are often confronted with disabilities on a physical, cognitive, social and/or communicative level. Rehabilitation of stroke patients is a time consuming process in which patients and caregivers have to learn new skills. In the Netherlands about 5,3 billion Euro are spend on rehabilitation of patients with cardiovascular diseases of which 1,5 billion is spend in nursing homes each year (4).

While it is reasonably established that the overall process of rehabilitation is effective, there is little evidence to support many specific rehabilitation therapeutic techniques $(3,5)$. Currently it seems that task orientated practice (i.e. practising an activity of relevance) is probably the most effective single therapeutic technique (6). This is not dissimilar to the situation in sport where practice is the bedrock of improving skills. Indeed improving any skilled motor activity seems to depend upon continuing practice. It is perhaps this similarity that has lead to using mental practice, a technique from sports, in neurological rehabilitation.

The use of mental practice or motor imagery is well established in sports (7-12). The principle is simple: a person imagines himself undertaking a skilled movement without actually doing the movement. It is a cognitive ability which is often used (un)consciously by all of us. Many therapists report that they use imagery in their therapy sessions already, but not systematically. They just ask the patient to imagine moving in a different way. Recently the use of mental practice has been subject to systematic research in neurological rehabilitation (13). Although many aspects of mental practice still remain unclear, it does seem to be a promising addition to the therapy, based on best evidence. Further research into mental practice is needed, particularly where it is embedded into normal rehabilitation services.

In the Netherlands, nursing homes provide a substantial amount of stroke care although most research is restricted to academic hospitals and rehabilitation centres (14). Consequently, more stroke research should take place in nursing homes.

The overall aim of the proposed research project is to investigate systematically the therapeutic potential of mental practice embedded in daily rehabilitation therapy on the improvement of daily activities of adult stroke patients compared to therapy as usual. 
The first additional research question is which prognostic variables or patient characteristics are associated with a positive outcome in the experimental subgroup.

The second additional research question investigates the feasibility of the mental practice-based therapy as judged by the patients and therapists.

This paper reports on preliminary work, and explains the choices made in the final study design, which has been approved by the medical ethical committee of the Atrium Medical Centre \& Maasland Hospital in Heerlen, the Netherlands.

\section{Background work}

A full systematic review of previous evaluative research on mental practice was completed and has been published (13). It showed some evidence suggesting that mental practice in stroke rehabilitation might work. However, the evidence still is weak. Furthermore, there is no consistency about or clarity on:

- the nature of the intervention itself (what was actually done?)

- the timing after stroke (when should patients be given mental practice?)

- control (what should be the control intervention?)

Consequently, we felt it important to set up a larger study. The review also highlighted several important issues to be considered in that:

- knowing what patients are doing during imagery (15),

- knowing whether patients are using mental practice (15),

- knowing what mental images of a movement the patient had (16),

- defining the core of mental practice (17),

- determining practicality in the commonest stroke rehabilitation setting (nursing homes in the Netherlands),

- determining what features predict success or failure.

These issues will be discussed also in this article. A multi-centre, prospective, parallel-group, randomised, controlled, observer masked trial (RCT) will be conducted over a two year period (mid 2007-mid 2009) involving clinically diagnosed adult stroke patients, complying with all normal research governance procedures. 


\section{Methods}

After recruitment of participants, a baseline measurement will occur (T0) followed by random assignment to either the control or experimental group. Effects of the interventions will be measured directly after the six weeks intervention period (T1) and six months after start of the intervention period (T2). Outcome measures will cover activities. Brain activity will be assessed with quantitative electro-encephalography (QEEG). An outline of the study design is given in figure one.

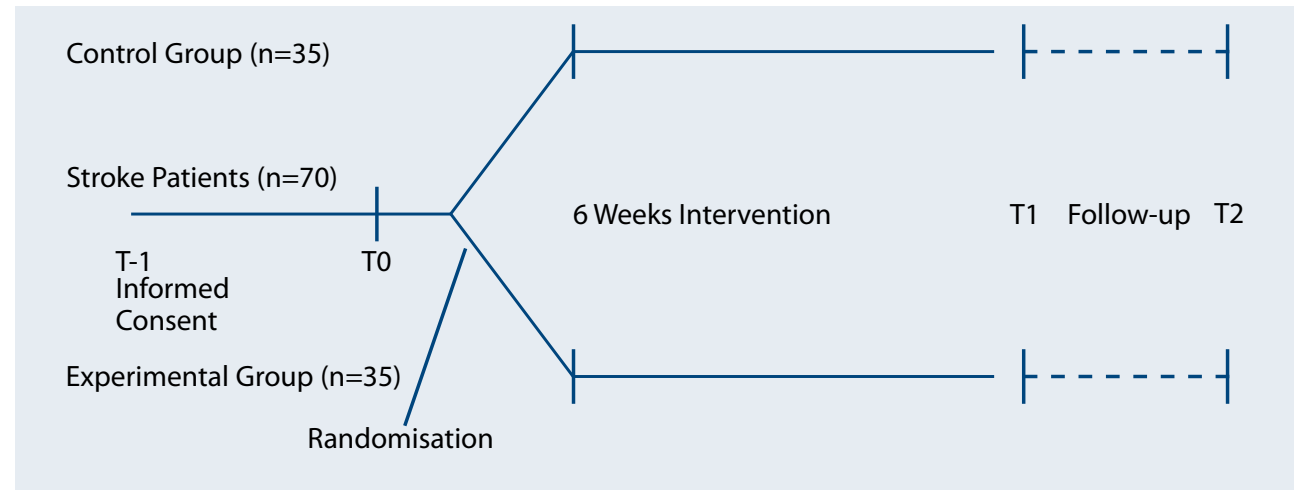

Fig.1: Outline of study design. $T-1=1-4$ days before baseline. $T 0=$ baseline $\mid T 1=6$ weeks $\mid T 2=6$ months.

\section{Study population}

Seventy stroke patients will be recruited in two nursing homes by the treating physicians or other staff. There are no data available concerning ability to benefit from mental practice, and so only practical factors affect inclusion and exclusion - can the person give consent and understand the instructions?

Patients entering the RCT have to meet following inclusion criteria:

a) clinically diagnosed adult stroke patient. In nursing homes, most of the stroke patients will be elder (>60 years old);

b) sufficient cognitive level and communication skills to engage in mental practice: this is a clinical judgment. Patients need to be able to follow simple instructions;

c) between two and 10 weeks of stroke onset.

\section{Exclusion criteria}

d) severe additional impairments prior to stroke causing persistent disability, like rheumatic diseases, orthopaedic problems after fall. 


\section{Sample size calculation}

The calculation of the sample size is based on the primary outcome measure, perceived improvement of daily activities, like 'drinking out of a cup' and 'walking', as assessed by an 11 point Likert scale. Although arbitrary, the calculation of the sample size is based on following expectations: For us a $20 \%$ difference between the two groups in this outcome measure would be a reasonable change to aim for which means a 2.2 point change on the Numeric Rating Scale $(\mu 1=2.2)$. There is no literature available on the standard deviation in a stroke population but in other populations the standard deviation on numerical rating scales is somewhere between 1.5 and 3.0. In this sample size calculation we will use a standard deviation of $2.25(\sigma=2.25)$. The power (the ability to detect a true difference in outcome) of this study is set at $90 \%(\beta=0.10)$. The level of significance (likelihood of detecting a treatment effect when no effect exists) is set at $5 \%(\alpha=0.05)$. The power and the level of significance are generally chosen by convention.

The sample size calculation formula used for measuring the difference between two unpaired samples is: $\mathrm{N} 1=\mathrm{N} 2=\left(\mathrm{z}_{1-\beta}+\mathrm{z}_{1-\alpha / 2}\right)^{2} *\left(\left(\sigma_{1}{ }^{2}+\sigma_{2}{ }^{2}\right) /\left(\mu_{1}-\mu_{2}\right)^{2}\right)$

This would mean that we would need to have 19 patients in each group assuming a 50:50 random allocation. The goal of this study is to have 35 patients in each group to allow for drop-outs, loss to follow-up and uncertainty in the power calculation. A sample size of $n=70$ seems realistically achievable over a 2 year period. Two nursing homes participate in this study: Klevarie nursing home (Maastricht) assesses about 200 new stroke patients each year. nursing home St. Camillus (Roermond) treats another 70 patients each year. We estimate that $40 \%$ of all treated stroke patients will be able to participate in the study, but not all will want to. If an added value of the experimental intervention cannot be found in 70 patients the clinical relevance of a mental practice-based therapy should be questioned.

\section{Treatment of participants}

All patients included in the study will receive six weeks of multi professional rehabilitation (2). Patients in the experimental group will receive their usual therapy extended with mental practice-techniques and principles embedded in therapy sessions. Paramedical therapists will be instructed (in the theory, in workshops and in training with their patients by an external expert $(\mathrm{SB}, \mathrm{MK}))$ on how to treat the patients in the experimental group. Patients allocated to the control group can be treated by any therapist. To prevent/limit contamination in therapy of the instructed therapists, an expert (also the trainer of mental practice for the participating therapists (SB)) will monitor the contrast between the experimental and control therapy by observing therapy randomly. 


\section{Experimental intervention}

First, we would like to make some general remarks on why we chose to embed mental practice in therapy and not give mental practice as an additional intervention outside of guided practice (e.g. with an audio tape). There is some evidence that mental rehearsal should be combined regularly with the overt movement to increase imagery vividness (17-20). Next, improving skills seems to depend on continuous practice $(2,3,5,21)$. Last, we believe that a higher training intensity will not only increase skills but also consolidate the mental practice technique, making the patient more confident that he/she is practising correctly and thereby increasing compliance and motivating patients to practice unguided.

The experimental intervention period is divided into four phases (fig. 2). In the first sessions, patients will first be familiarized with mental practice-based therapy and educated by their treating therapists as to basic imagery principles and the importance of imagery training on a regular basis (phase 1). There is some evidence that patients educated on and familiarized with the technique are more likely to practice in general and to practice correctly by themselves (22-24).

\section{Mental Practice Training}

(6 weeks)

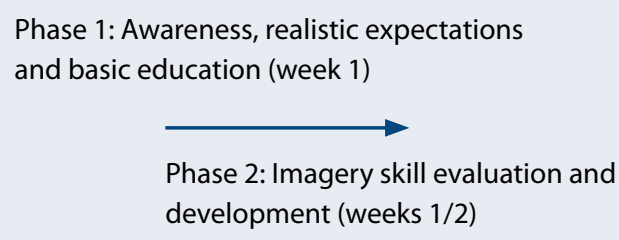

Phase 3: Using imagery to improve activities (Use at home) (weeks 3 to $6+$ unguided $^{*}$ )

Phase 4: Imagery evaluation, adjustment and refinement (week 6)

Fig.2: Overview of Mental Practice training phases and their aims.

* at least 3 times 10 minutes a day. Preferably, mental practice is combined with physical or occupational therapy or the overt movement at lunch time. Time spend on mental practice unguided can be increased considerable due to compliance/motivation of the patient to practice. Training data are recorded in a personal log (diary) preferably by the patient or assisted by a member of the family or therapist/nurse. 
During the next one to two weeks, phase 2, they will be taught by their treating therapist how to use the mental practice technique to improve 'drinking from a cup' (occupation therapy) and/or 'walking - with(out) walking aids' (physiotherapy), depending on what they want to improve. The main reason that we chose these two activities is that patients report these activities most frequently, as activities they want to improve when starting rehabilitation. We also wanted two common activities for all patients to practice so that we can standardize the learning process and we will be able to compare results, at the end of the study. Third, 'drinking from a cup' and 'walking' are different kind of tasks: walking is repetitive with timing of different body segments (arm swing, body rotation combined with leg swing), whereas drinking is a 'forward-back movement', where the different parts of the movement seem to follow each other up in time.

Perhaps these movements involve different amounts of cortical information. This could imply that certain movements are more suitable to practice mentally for they need more cortical involvement (attention) for a successful performance. Within the speech therapy, mental practice will be used to improve 'swallowing problems' or motor problems due to facial pareses, like 'smiling' symmetrically. We see no direct plausibility to improve aphasia with mental practice and have found no evidence for it in literature.

We will use results from the Structural Dimensional Analysis of Motor memory (SDA-M) (17, $18,20)$ to determine the basic architecture of specific goal-directed movements and thus to guide the imagery given through identifying inappropriate mental processes. It is for example used to identify weak spots in the sequence of events that should lead to a certain motor performance in sports.

We have investigated the reproducibility and feasibility of the SDA-M in a nursing home stroke population for the motor action 'drinking out of a cup'. The measuring protocol was successfully adjusted to the ability of the stroke population to process information. The measure instrument seems useful in rehabilitation (16). The SDA-M results will be performed and interpreted by an external expert $(\mathrm{SB})$ and used by the treating therapists to tailor the mental practice intervention of individual patients in the experimental group.

The vividness of imagery will be enhanced using videos of the tasks and stimulating patients to recall all sensory information during imagery, like sounds, smell, touch, pressure and taste.

The duration of the first two phases can differ depending on how fast the patient catches on to the technique. It will however not take longer than two weeks. 
During the four weeks training period (phase 3), patients will receive guided mental practice-based therapy in their routine rehabilitation. Movement imagery will be alternated with overt movement in short individually determined blocks (for example: three times a mental rehearsal of the movement, three repetitions of the overt movement). These blocks of imagery and motor action will be interrupted by short breaks (one minute). The embedded imagery training will take place in the first 10-15 minutes of 30 minutes treating time when patients are still fresh and alert. Neural (coordination) training should take place when the nervous system is fresh (participants should not be tired due to physical exercise). Participants will be encouraged to practice unguided as much as they want. The unguided training amount is registered daily in a log. The logs are used to discuss progress in unguided therapy at the beginning of a therapy session. In a log the participants can record one week of unguided training, a new one is handed over after every week.

Three refreshment sessions can be held in which tasks are shown on a video or demonstrated. The SDA-M may be repeated if the treating therapist thinks this is useful, for example if significant change has occurred. The external expert (SB) will perform the test and again the results will be used to adjust the content of the mental practice intervention. Apart from optimizing the mental practice of 'drinking from a cup' and / or 'walking' the aim of the refreshment session is to add additional tasks (one more task per profession). This way, additional activities the patients want to improve are part of the protocol as well.

In the fourth phase, a general evaluation will take place to see whether any adaptations, advice or alterations are necessary in order for the patient to continue mental practice at the nursing home or at home. This will take no longer than two therapy sessions.

\section{Control intervention}

The control group will receive therapy as usual in accordance with the Dutch guidelines for stroke rehabilitation $(2,3,21)$. To compensate for the unguided imagery training, patients in the control group will be encouraged to do 'homework' as well, primarily practising tasks that they find difficult. Participants in the control group will also be instructed to use logs.

\section{Study parameters/endpoints}

Measurement dates are at entry in the study (T0 - baseline), after a six weeks intervention period (T1) and six months after entry (T2). Additional information during the course of rehabilitation will be collected, e.g. recurrence of stroke, other intercurrent medical problems, amount of therapy received, used medication. In addition, any deviation from the treatment protocol and any co-intervention during the six months will be recorded. 
In the choice of measuring instruments following considerations were taken into account:

- the measuring instruments should have sufficient methodological quality and feasibility recorded in literature and/or be recommended by the national (Dutch) stroke guidelines;

- if possible, the measuring instrument should be part of the standard assessment in the nursing homes to restrict the additional load on the patient;

- if the quality of a listed measuring instrument has not yet been established the measuring instruments should have evidence from other studies on their potential use in stroke rehabilitation;

- the feasibility will be measured by using semi-structured interviews (process evaluation), recording co-interventions, analysing logs and monitoring therapy content in the experimental group.

The extra load for the participants due to additional clinical testing is approximately 20 minutes at each assessment point.

\section{Demographics}

Upon the patient's entry (T0) into the study the full neurological status and following patient characteristics will be recorded: age, gender, brain lesion site (from status), extent of hemiplegia (the perceived disability as categorized by the patient: no, mild, average and severe disability), time post stroke, ability to imagine motor acts (as perceived by the patient in four categories: no ability, blurry/unfocussed, some/ parts, vivid imagery), cognitive level (MMSE), hand dominance prior to stroke. Additional information about highest educational level and sports history (no history, leisure, competition, profession) by asking the patient is recorded as well. Treating therapists are asked to predict, based on their experience, whether patients in the experimental group will benefit from a mental practice based therapy.

We will then investigate whether the two groups are comparable and whether any of these data have prognostic value.

\section{Main study parameter/endpoint}

It is hypothesized that mental practice has the most effects on the movement that is actually mentally rehearsed $(9,18,25,26)$. Improvement of these activities should therefore be assessed. To measure if mental practice improves the performance of activities in the experimental group more than in the control group, an 11 point Likert scale will be used:

11 point Likert scale assesses the performance of the activities 'drinking' and 'walking' ranging from 10 ('excellent') to 0 ('poor') as perceived by the patient and the therapist. 
The physiotherapist and the occupational therapist will each score another task, which may be individually chosen and scored on the 11 point Likert scale. If the participant is treated for swallowing problems and/or motor problems due to a facial paresis by a speech therapist, the patient and the speech therapist will score motor actions within these two domains on perceived performance.

\section{Secondary study parameters/endpoints}

- Motricity Index (MI - function and activity level)

The Motricity Index evaluates voluntary movement activity and the maximum muscle strength with a six point scale in six limb movements. Reliability and validity are sufficient in stroke populations (27).

- Barthel Index (BI - function and activity level)

With the Barthel Index the degree of independent performance of daily activities is measured (28). Several versions exist. In this study an assessment form with a 20 points scale will be used (28).

- Nine Hole Peg Test (NHPT - function and activity level)

The NHPT is a measuring instrument in which the speed of the fine hand coordination is assessed. The patient has to take nine dowels from a tray, one at a time, as fast as possible and place them in a pegboard. The time needed to complete the attempt is recorded. Only the hand that is being assessed (i.e. the affected hand) may be used. The reliability and validity are sufficient (29-31).

\section{- Rivermead Mobility Index (RMI - activity level)}

This is a staff-completed questionnaire to measure mobility disability after head injury, multiple sclerosis (MS), stroke and other conditions. It comprises of 14 questions (activities scored range from turning over in bed to running) and one direct observation of standing for 10 seconds. Each answer is scored 'yes' (1) or 'no' (0). The minimum score is 0 and the maximum score is 15 . The higher the score is, the better the mobility.

- 10 meter walking test (TMW - activity level)

The 10-meter walking test can be used in patients able to walk independently with or without walking aids and/or orthoses. Patients should walk at a comfortable speed. The test is reliable, valid and responsive (27). Furthermore, a significant relation between the comfortable walking speed during the TMW and the quality with which patients walk has been established (32). Codes for not able (yet) and independent in wheelchair are 0 and. 1 . 
- Timed up and go (TUG - activity level)

The TUG measures the time a patient needs to stand up from a chair, walk 3 meters at a comfortable speed, turn around, walk back and sit down. The patient is allowed to use his/her own walking aids, but no physical assistance may be given by the researcher or therapist. The test is practical and simple. The internal consistency, reliability, validity and responsiveness are sufficient (33-37).

\section{Optional study parameters}

QEEG (Brain-activity - neurophysiological level)

EEG activity in stroke is primarily assessed in the acute phase of stroke recovery to reveal possible epileptic activity (38). QEEG assessment in the acute and chronic phase of recovery is not a generally performed procedure, although it might function as a reliable marker for monitoring the recovery and predicting the clinical outcomes after stroke $(39,40)$. In numerous studies, brain electrical activity across the sensorimotor cortex has been related to both execution and imagination of movements. The mu rhythm $(8-15 \mathrm{~Hz})$ seems to be connected to movement in general and is only to be found above the sensorimotor cortex (41). In general, body movements block or suppress mu activity up to $60 \%$, and imagination of movements generates similar suppression. It is hypothesised, that alterations in mu rhythm suppression during motor imagery in stroke would reflect distorted information processing of the sensorimotor cortex, thereby functioning as a possible marker for decreased ability of imagination of movements (42). In the RCT, we want to investigate if the QEEG can be used as a biomarker to predict if patients are able to perform imagery and are likely to benefit from a mental practice based intervention.

In addition to the QEEG as a prognostic value, the mu suppression is used as an evaluative measure to assess progress in imagery techniques during the six weeks intervention period.

Suppression of the mu waves can be interpreted as movement related information processing. Measures of brain activity will be performed with a universal amplifier (MPAQ, Maastricht Instruments, the Netherlands) and data acquisition software (IDEEQ, Maastricht Instruments, the Netherlands). Eight sensors will be placed above the sensorimotor cortex at both hemispheres according to a standardized protocol. To ensure low skin impedance $(<5 \mathrm{k} \Omega)$, the skin will be cleaned with a lotion and a non-allergic gel will be used for better transmitting of the signal (Ten20 conductive gel). Results will be expressed in \% of suppression of mu activity. Patients may refuse QEEG measures at T1 and T2 due to the additional load of 20 minutes per assessment. If necessary due to allergy, nickel-free electrodes will be used. 


\section{Compliance, integrity check and feasibility of mental practice}

During the mental practice intervention period, therapy compliance of the patient is monitored by using a log in which the time spent on practice is recorded on a daily basis. The diary entries will be checked by either the therapist or a member of the nursing staff. In the Dutch guidelines the amount of therapy is considered of importance with regard to effectiveness of any intervention to improve functions $(2,3,21)$.

Furthermore, a small sample of the participants will be interviewed by the researcher on experiences and beliefs during mental practice in the experimental group and on content of therapy as usual in the control group ( $\mathrm{n}=10$ in each group for both sites). These will be selected arbitrarily, and limited to those agreeing.

The therapist will be monitored as well. They will be asked to what extent they followed the given instructions from the mental practice protocol. All therapists will be interviewed on their opinion of the feasibility of the experimental intervention in every day practice. To monitor the intervention content an external expert will attend therapy sessions of participants in the experimental group unannounced. Table one (next page) summarizes the data collection.

\section{Randomisation, blinding and treatment allocation}

No stratification will take place (43). Randomisation will take place on the participant level. Based on a computerized (block) randomisation schedule with random block size (4 or 6) seventy sequentially numbered envelopes will be prepared, with equality being achieved after every four or six. Each participant recruited will be registered and given the next sequential number, and then the envelope will be opened to determine their allocation. The randomisation procedure is the same for both sites. 
Tab.1: Overview of used measures in this study.

\begin{tabular}{|c|c|c|}
\hline Data & Time & Subject of assessment \\
\hline \multicolumn{3}{|l|}{ Demographics } \\
\hline Age, gender, time post-stroke, weak side... & T0 & \multirow[t]{4}{*}{ Comparison at baseline } \\
\hline MMSE & T0 & \\
\hline Psychological assessment & T0 & \\
\hline Therapist' prediction on mental practice performance & T0 & \\
\hline \multicolumn{3}{|l|}{ Prognostic variables } \\
\hline Amount of therapy/training & T0 & \multirow[t]{2}{*}{ Prediction on outcome } \\
\hline QEEG & T0 & \\
\hline \multicolumn{3}{|l|}{ Primary outcome } \\
\hline $\begin{array}{l}11 \text { point Likert scale: drinking and walking/two } \\
\text { patient specific tasks }\end{array}$ & $\mathrm{T} 0, \mathrm{~T} 1, \mathrm{~T} 2$ & $\begin{array}{l}\text { Performance on physical level } \\
\text { 'activity' }\end{array}$ \\
\hline \multicolumn{3}{|l|}{ Secondary outcome } \\
\hline Motricity Index & $\mathrm{T} 0, \mathrm{~T} 1, \mathrm{~T} 2$ & \multirow{3}{*}{$\begin{array}{l}\text { Performance on physical leve } \\
\text { 'function' }\end{array}$} \\
\hline Barthel Index & $\mathrm{T} 0, \mathrm{~T} 1, \mathrm{~T} 2$ & \\
\hline Nine Hole Peg Test & $\mathrm{T} 0, \mathrm{~T} 1, \mathrm{~T} 2$ & \\
\hline Berg Balance Scale & $\mathrm{T} 0, \mathrm{~T} 1, \mathrm{~T} 2$ & \multirow{5}{*}{$\begin{array}{l}\text { Performance on physical level } \\
\text { 'activity' }\end{array}$} \\
\hline Rivermead Mobility Index & $\mathrm{T} 0, \mathrm{~T} 1, \mathrm{~T} 2$ & \\
\hline Ten metres Walking Time & $\mathrm{T} 0, \mathrm{~T} 1, \mathrm{~T} 2$ & \\
\hline Timed Up and Go & T0, T1, T2 & \\
\hline Functional Ambulation Category & T0, T1, T2 & \\
\hline \multicolumn{3}{|l|}{ Optional } \\
\hline QEEG & $\mathrm{T} 1, \mathrm{~T} 2$ & Performance on brain activity \\
\hline During intervention period & Daily & \\
\hline $\begin{array}{l}\log \\
\text { Interview }\end{array}$ & Once & $\begin{array}{l}\text { A small sample }(n=10) \text { will } \\
\text { be interviewed to assess the } \\
\text { patients opinion on feasibility } \\
\text { of the program. }\end{array}$ \\
\hline Co-interventions & $\mathrm{T} 1, \mathrm{~T} 2$ & Process evaluation \\
\hline
\end{tabular}

\section{Blinding}

At baseline, before randomisation the measurements will be performed by the treating therapists and psychologist of the staff. The measurements at T1 and T2 will be performed by an independent trained rater. The patients are aware of the treatment they receive, so it is not possible to blind them. The rater however will be blinded for the treatment allocation: patients will be asked by the rater not to reveal the treatment to which they were assigned. A blinding 
check will be performed after each of the two measurement sessions (T1 and T2). A process measure as to success of rater's blinding is the rater's opinion about the group he thinks a patient belonged to.

\section{Withdrawal of individual participants}

Participants can leave the study at any time for any reason if they wish to do so without any consequences. If reasons are given they will be recorded. The investigator can decide to withdraw a subject from the study for urgent medical reasons. We allowed for a $20 \%$ drop out in the size calculation.

\section{Follow-up of participants withdrawn from treatment}

The statistical analyses will be performed according to the 'intention to treat' principle (patients will be analysed in the treatment group to which they were randomly assigned). Patients who withdraw from treatment but allow further data collection will have data collected.

\section{Statistical analysis}

All data will be collected on paper and the records will be stored by registration number in a secure cabinet. Anonymised data will be transferred to a computer database and secured using a password. Entries in the patients' diaries and results from the interviews will be analysed qualitatively.

\section{Multivariate analysis}

The baseline scores of the patient's demographic, primary and secondary outcomes will be used to compare the two groups. If necessary, adjustments for baseline variables will be made, using analysis of covariance. Differences at baseline and differences between the two groups on the various assessment times will be calculated. Data will be analysed using MAN(C)OVA and generalized estimating equations (GEE) analyses to ascertain the effects of mental practice based therapy on different levels of outcome and to follow improvements individually in time. Regression analyses will be used to identify prognostic variables. The statistical analysis concerned with comparing the two groups will be performed according to the 'intention to treat' principle (patients will be analysed in the treatment group to which they were randomly assigned). Missing data will be replaced by a linear interpolation method for missing measurements. A 'last measurement carried forward' method is used to predict outcome in dropouts (44). 


\section{Ethical considerations}

Measurement instruments have been chosen as far as possible from the assessment protocol of the nursing homes, thereby minimizing the additional testing load during intake and testing for the individual patient. Table two gives an overview of the main ethical considerations.

Tab. 2: Overview of the ethical considerations.

\begin{tabular}{l|l}
$\begin{array}{l}\text { Question } \\
\begin{array}{l}\text { How great was the change } \\
\text { in clinical practice? }\end{array}\end{array}$ & $\begin{array}{l}\text { Minor: use of consistent advice and consistent technique in both } \\
\text { groups (embedded in therapy as usual) }\end{array}$ \\
$\begin{array}{l}\text { What extra burden } \\
\text { was imposed upon the } \\
\text { patient(s)? }\end{array}$ & $\begin{array}{l}\text { Moderate: some time in collecting data from measures not standard in } \\
\text { the protocols of the nursing homes. }\end{array}$ \\
$\begin{array}{l}\text { What additional risks did } \\
\text { the patient(s) (or other } \\
\text { participants) face? }\end{array}$ & $\begin{array}{l}\text { Minor: from the mental practice intervention none can be thought of } \\
\text { at present, results of QEEG could generate some ethical issues for the } \\
\text { researcher if major unexpected abnormalities are discovered }\end{array}$ \\
$\begin{array}{l}\text { What benefit might accrue } \\
\text { to the patient (or other } \\
\text { participants)? }\end{array}$ & $\begin{array}{l}\text { Moderate: experimental treatment may be of complementary value to } \\
\text { current practice }\end{array}$ \\
$\begin{array}{l}\text { What benefit might accrue } \\
\text { to Society? }\end{array}$ & $\begin{array}{l}\text { Moderate: the study should detect any clinically relevant difference } \\
\text { in treatment. Papers in peer reviewed journals will be submitted and } \\
\text { researchers will learn and teach in research methodology }\end{array}$ \\
$\begin{array}{l}\text { Was each participant } \\
\text { informed about the } \\
\text { study and able to choose } \\
\text { whether or not to } \\
\text { participate? }\end{array}$ & $\begin{array}{l}\text { The patient is informed orally and in writing. Participation in the study } \\
\text { may be considered for at least } 2 \text { days. Patient may withdraw from } \\
\text { the study at any time without giving reason why. This will not affect }\end{array}$ \\
$\begin{array}{l}\text { Was the method of } \\
\text { treatment negatively }\end{array}$ \\
$\begin{array}{l}\text { recruiting participants fair } \\
\text { and appropriate? }\end{array}$ & $\begin{array}{l}\text { As little is known about specific selection of stroke patients likely to } \\
\text { benefit most from a mental practice regime in rehabilitation, inclusion } \\
\text { criteria were kept as broad as possible }\end{array}$ \\
\hline
\end{tabular}

\section{Recruitment and consent}

The method of recruitment should be fair, neither disadvantaging some patients nor advantaging others. All patients will received full information and will be given at least 48 hours to decide whether they wish to participate in the research, and will be able to withdraw at any time without affecting their other rehabilitation. Witnessed consent will be obtained. Patients may contact an independent physician for information and advice. The patient is free to refuse participation.

\section{Benefits and risks assessment, group relatedness}

As there are no invasive interventions, nor any untested experimental measurement instruments used, there is no additional risk associated with the additional assessments or treatment of the patient. There will be a small extra burden potentially placed on patients. They will spend more 
time seeing the researcher. They will be asked to answer questions, fill in questionnaires, and sometimes to undertake activities or tasks that might be timed. However none of this will be especially uncomfortable or troublesome, and all can be undertaken by the patient at their own pace.

The risks faced by patients are no greater than those risks they face during routine rehabilitation practice. The only potential exception to this is the QEEG. A QEEG in itself carries no direct risks. Theoretically, a patient might be allergic to the materials used, but each patient will be asked if they are allergic to these materials (e.g. the metal involved in the electrode) and a anti-allergic gel will be used to improve signal measures.

\section{Discussion}

This study poses several interesting problems when considering its design. When we started out deciding how the trial should be performed, we first discussed the goals of our research. We thought it important that mental practice should be applicable to as many stroke patients and usable in as many settings as possible. This meant that the costs for the program should be low, therapists should be instructed easily and the content of mental practice should not be difficult to follow, so that not too many exclusion criteria should be set. We then decided to run the study in nursing homes, because they have a major role in stroke rehabilitation in the Netherlands (14). The choice for this setting has many implications for the content of the experimental intervention and the recruitment of eligible participants. Two important aspects of recruitment concern the selection criteria and the best time to approach the patients. Our third main concern was to define the control intervention and the outcome measures in order to identify effects of mental practice on stroke recovery. These aspects will be discussed below.

Strategy versus tactics: teach how to do mental practice in general, or teach only a specific task? Although all nursing homes work according to the best available evidence, there are considerable differences in tasks and organization of the nursing homes, depending for instance if they are part of specific stroke services or not. This reflects in variations in time post stroke at submission, the neurological assessments, tasks of professionals and routing of the patient during the rehabilitation process and also in the duration of the rehabilitation process. We wanted to create an intervention protocol that could be used in any nursing home, independent of the organization form.

Second, we wanted therapists of different disciplines to use mental practice in motor learning tasks of the same patient, so that they could reinforce each other's therapy. 
We therefore made the explicit choice to only set a fixed theoretical framework for using mental practice, but also leave room to tailor therapy to the preferences and abilities of the patient, thereby giving the professional space to use his/her experience (real practice) instead of forcing patients into a fixed intervention program.

\section{Incorporation into routine working practice}

In reported studies, sometimes the physiotherapist, the occupational therapist or the psychologist instructed and guided the patients through the mental practice and sometimes an audio tape was used for this purpose (13). There is no evidence that a certain caregiver is a better mental practice coach than another. As stated before we chose to incorporate mental practice into every paramedical therapy session. To ensure that all treating therapists of the experimental group were informed of what the other therapists were doing and to enhance and use imagery skills of a patient, all physiotherapy, speech therapy and occupational therapy sessions were recorded in one central log. If time does not allow the therapists to discuss patients in person, they are still able to see what others have done and use this information for their own sessions.

In order to use their expertise for tailoring mental practice, a therapist should have practical experience in teaching and monitoring it in daily routine. Therapists need to be instructed thoroughly, not only theoretically but especially with regard to 'hands on' skills. Therefore, a great part of the instruction needs to consist of workshops (showing and giving examples) and training on the job (guiding). Sufficient time needs to be reserved for therapists to get familiar with mental practice and teaching it confidently. We incorporated a preliminary phase of about five months for the therapists to get comfortable with mental practice, gaining experience and incorporating it into routine working practice, before starting the trial.

Because we randomised on the patient level (and not on for instance institute or therapist level) and because we trained all therapists involved in the trial (giving both the experimental and control intervention), we faced a challenge: avoiding possible contamination of mental practice into the control content. We choose for randomisation on patient level because we felt that real care of patients should come first (see: selecting patients). We trained all therapists to maximize recruitment within the nursing homes. Both therapists and patients can diminish the contrast between the experimental and control intervention.

Caregivers who were made enthusiastic about a new approach and have just spend a lot of time learning how to use mental practice as an additional tool may be tempted to use it as much as possible, whenever possible. This could decrease the differences between the two compared 
interventions. There is some evidence that altering professional behaviour is not very easy (45). Therapists will probably need more effort to consciously implement mental practice into their daily routines then copying them in the control group. Nonetheless, therapists will undergo an integrity check too, determining how well they were able to distinguish between the two interventions in therapy. During an interview, therapists will be asked to which extend they were able to stick to the intervention contents (subjective opinion). Furthermore, the external expert will attend therapy sessions of all therapists unannounced and at random throughout the two year trial period. With these measures, we will try to maximize the contrast between the compared interventions. In case contamination does take place, we will report it as fair as possible.

From the patient's point of view, we believe there is a big difference between telling patients to imagine movements and actually teaching it. It is more probable that patients that were taught will successfully use mental practice on a regular basis. A big part of the effect will probably come from the practice outside of therapy, unguided.

\section{Selecting patients}

Ethical considerations in recruiting patients should always be taken in account, especially if so little is known about effects and prognostic values, as is the case in imagery research. Because we do not know whether mental practice will lead to better and/or quicker recovery in this research the only 'ethical' design seems to be a randomised trial. Data analysis with GEE will give us more ideas on improvements on an individual basis.

Patients unable to learn imagery should not be frustrated unnecessary, but we also do not want to withhold mental practice from patients who might well benefit from mental practice. Since we do not know who might benefit from mental practice we believe it ethical to include anyone able and willing to participate in the research. Within this study, the participation judgment is made based on the extend of attention a patient has, as well as the amount of working memory he/she has and the ability to perceive different information. Participants need to be able to think practical but analytical about a movement. Predictions of the treating therapists on the ability of the patient to participate in the study will be discussed during multi disciplinary meetings of the paramedical team and a prediction of the patient's ability to participate in mental practice will be made based on consensus.

We are aware that patients who just have had a stroke are extremely vulnerable and should be given time to cope with their new situation. So when should recruitment start? In preliminary 
work, we found that about $25 \%$ of the acute stroke patients (one to two weeks post stroke) in the nursing homes were willing to participate in a pilot study. We decided to start recruitment within the RCT at any time between two and ten weeks post stroke which should maximise recruitment while maintaining the focus on patients in the acute phase. Those patients who are recovering well and able to start earlier will probably start at two-three weeks but patients who are sicker or slower in recovering still have an opportunity to start up to ten weeks. This approach should, oddly, reduce heterogeneity in the population studied because each person will be recruited at a similar stage in their own recovery trajectory.

\section{Knowing if people are practising}

Two aspects are important with regard to compliance of the patient to the experimental intervention. 1. Are patients actually thinking about movements during mental practice and 2. Are they reporting the unguided therapy time correctly?

At this point, we do not know of any measure that can measure thoughts. In different studies, the main way to check if participants are imaging movements is the indirect way of interviewing. Our experience is that one can get a good idea of what the patient has been doing, just by asking. We therefore chose to use semi-structured interviews in our trial to check on what patients are doing during imagery. However, we also wanted a quantitative measure to verify self-reports. The idea of using QEEG as a biomarker for imagery came from a study Ramachandran performed with autistic children (46). Mu wave suppression seems to be a key factor in determining whether a participant is imagining movement or not. In the trial, we want to assess if mu-wave suppression during imagery is trainable and whether it can be used as a prognostic value in the nursing home stroke population.

One of the benefits of mental practice is that it can increase the therapy amount considerably: patients are not dependent on time spend in the gym with therapists, but can exercise safely any time, anywhere $(22,23,47-56)$. This results in giving the patient more autonomy over his / her rehabilitation process, which might well motivate certain participants to practice.

Therapy amount could well be a prognostic variable for outcome. It is therefore important to register unguided therapy time as detailed as possible. A log was developed for this purpose. In it, the patient can describe on a daily basis, what activity he/she has practiced mentally, for how long and how well it went. Additionally the patient can score how he/she felt that day by marking one of three smiley's (bad day, neutral day, good day). At the bottom of each page (day), space is left open for comments and remarks, for instance to report if anything unusual had happened. To encourage patients to use the log, every session starts with looking at the log's 
entries and discussing them with the patient. By involving family members and the nursing staff, we hope to come as near as possible to the actually practiced time.

\section{Control for mental practice training}

By embedding mental practice in the regular therapy sessions and therapy time, we tried to minimize the additional training time in the experimental group. About four to six additional hours are made during the six weeks intervention time (one hour per week). The majority of these six hours are spend on performing the SDA-M in the second phase of the mental practice protocol (three-four hours) during regular therapy time. The external expert will perform this assessment and discuss outcome with the treating therapists. The other two to three hours are used instructing participants on what mental practice is, tailoring the intervention to the patient's abilities and preferences and explaining the log as well as interviewing a part of the patient group.

We decided to compare mental practice embedded in therapy with therapy as usual (TAU) and compensate for extra time spend with patients and given attention were possible. The SDA-M is not performed in the control group. During these hours, participants from the control group will have therapy as usual.

To compensate for attention, participants from the control group will be instructed to keep a log on their homework (movements) and then score the actual performance of these actions. Within the control group an equally big sub group will be interviewed on their perceptions and believes with regard to the control intervention.

\section{Outcome measurement}

There is little known about what specific aspect or combinations of aspects of mental practice lead to positive results $(18,19)$. Outcome measures within neurological studies are often chosen to assess physical improvement, but imagery can also have effects on motivation and altering cognition and feelings, like anxiety. Martin and co-workers developed an applied model, based on models from cognitive psychology, to provide a guiding framework that describes how an athlete can use imagery to achieve a variety of cognitive, affective, and behavioral changes across different sport situations (17). It is very important that such a (for neurological rehabilitation) adjusted model were applied in neurological rehabilitation as well, to ensure future comparison of studies.

We specifically chose to use mental practice to improve physical performance, trying to leave mental practice content that has effects on other domains, out of the intervention protocol. 
The measuring instruments were chosen with this information in mind. We thought it very important for patients to be able to choose the tasks they wanted to improve, for they should be relevant for the client. Just as in athletes, patients then use mental practice for specific motor actions. Exactly these movements should then improve. The chosen tasks, apart from 'drinking from a cup' and / or 'walking', are likely to vary greatly, since they depend on the patient's preferences. The 11 point Likert scale was chosen as the primary measure because it seems most plausible that effects of mental practice should first and mainly be detectable on the rehearsed tasks. Second, we wanted one measure that could be used for any kind of motor action. Subjective changes in the performance of the task from both the therapist's view as the patient's view seem equally important. The secondary measures were chosen to reflect on as many tasks as possible.

\section{Final remark}

By describing the design, we hope to contribute to the discussion on how mental practice should be introduced in stroke rehabilitation. Results from this study, might clarify some aspects of mental practice in stroke rehabilitation and give further leads on how to do it, or how not to.

\section{Acknowledgements}

We would like to thank Melanie Kleynen, Maartje Schoenmakers and Björn Crüts for the interesting discussions and sharing thoughts on the design.

\section{References}

1. American Heart Association: Stroke facts. Dallas: AHA; 1997.

2. Commissie CVA-revalidatie: Revalidatie na een beroerte, richtlijnen en aanbevelingen voor zorgverleners. Den Haag: Nederlandse Hartstichting; 2001.

3. van Peppen RP, Kwakkel G, Harmeling-van der Wel BC, et al.: KNGF-richtlijn beroerte. Ned Tijdschr Fysiother 2004, 114(Suppl):1-77.

4. RiVM kosten van zieken [http:/ / www.rivm.nl/vtv/object_class/kvz_diagnose_ hartvaatstelsel.html.] 2007.

5. Kwakkel G, van Peppen R, Wagenaar RC, et al.: Effects of augmented exercise therapy time after stroke: a meta-analysis. Stroke 2004;35:2529-39.

6. Trombly CA, Wu CY: Effect of rehabilitation tasks on organization of movement after stroke. Am J Occup Ther 1999;53:333-44.

7. Bakker FC, Boschker MS, Chung T: Changes in muscular activity while imagining weight lifting using stimulus or response proportions. J Sport Exerc Psychol 1996;18:313-24. 
8. Feltz DL, Landers D: The effects of mental practice on motor skill learning and performance: A meta-analysis. J Sport Psychol 1983;5:25-57.

9. Feltz DL, Landers D: A revised meta-analysis of the mental practise literature on motor skill learning and performance. In: Enhancing human performance: issues, theories and techniques. Druckman D, Swets J. editors Washington (DC): Natl Acad Pr; 1988. p 1-65.

10. Janssen JJ, Sheikh AA: Enhancing athletic performance through imagery: an overview. In Imagery in sports and physical performance. Edited by: Sheikh AA, Korn ER. Farmingdale: Baywood;1994: p 1-22.

11. Morris T, Spittle M, Watt A: Imagery in Sport. Champaign: Human Kinetics; 2005.

12. Porter K: The mental athlete. Champaign: Human Kinetics; 2003.

13. Braun SM, Beurskens AJ, Borm PJ, Schack T, Wade DT: The effects of mental practice in stroke rehabilitation: a systematic review. Arch Phys Med Rehabil 2006;87:842-52.

14. Schols JM, Crebolder HF, van Weel C: Nursing home and nursing home physician: the Dutch experience. J Am Med Dir Assoc 2004;5:207-12.

15. Sharma N, Pomeroy VM, Baron JC: Motor imagery: a backdoor to the motor system after stroke? Stroke 2006;37:1941-52.

16. Braun SM, Schack T, Marcellis RG, Oti KC, Schols J, Wade DT, Beurskens AJ: Is it possible to use the SDA-M to investigate representations of motor actions in stroke patients? Clin Rehabil 2007;21:822-832.

17. Martin K, Moritz S, Hall C: Imagery use in sports: A literature review and applied model. Sports Psychol 1999, 13:245-68.

18. Budney AJ, Murphy SM, Woolfolk RL: Imagery and motor performance: what do we really know? In Imagery in sports and physical performance. Sheikh AA, Korn ER. Farmingdale: Baywood: 1994. p 97-120.

19. Driskel JC, Copper C, Moran A: Does mental practice enhance performance? J Appl Psychol 1994;79:481-92.

20. Gould D, Damarjian N, Greenleaf C: Imagery training for peak performance. In Exploring sport and exercise psychology. Edited by: Raalte van J, Brewer WB, editors, Washington (DC): Am Psychol Assoc 2002. p 49-74.

21. Cup E, Stultjens EM: Ergotherapie richtlijn beroerte. Utrecht: Nederlandse Vereniging Ergotherapie; 2005.

22. Liu KP, Chan CC, Lee TM, Hui-Chan CW: Mental imagery for relearning of people after brain injury. Brain Inj 2004;18:1163-72.

23. Lui KP, Chan CC, Lee TM, Hui-Chan CW: Mental imagery for promoting relearning for people after stroke: a randomised controlled trial. Arch Phys Med Rehabil 2004;85:1403-8. 
24. Stevens JA, Stoykov ME: Using motor imagery in the rehabilitation of hemipareses. Arch Phys Med Rehabil 2003;84:1090-2.

25. Boschker M: Action-Based Imagery. On the nature of mentally imagined motor actions. [Dissertation] Amsterdam : Vrije Universiteit Amsterdam IOpskamp PrintPartners; 2001.

26. Hall C: Imagery and the acquisition of motor skills. Can J Sports Sci 1992;17:19-27.

27. Garraway WM, Akhtar AJ, Prescott RJ, Hockey L: Management of acute stroke in the elderly: preliminary results of a controlled trial. Br Med J 1980, 280:1040-3.

28. Kalra L, Eade J: Role of stroke rehabilitation units in managing severe disability after stroke. Stroke 1995;26:2031-4.

29. Goodkin DE, Hertsgaard D, Seminary J: Upper extremity function in multiple sclerosis: improving assessment sensitivity with box-and-block and nine-hole peg tests. Arch Phys Med Rehabil 1988;69:850-4.

30. Oxford Grice K, Vogel KA, Le V, et al.: Adult norms for a commercially available Nine Hole Peg Test for finger dexterity. Am J Occup Ther 2003;57:570-3.

31. Smith YA, Hong E, Presson C: Normative and validation studies of the Nine-hole Peg Test with children. Percept Mot Skills 2000;90:823-43.

32. Hankey GJ, Deleo D, Stewart-Wynne EG: Stroke units: an Australian perspective. Aust N Z J Med 1997;27:437-8.

33. Lin MR, Hwang HF, Hu MH, et al.: Psychometric comparisons of the timed up and go, one-leg stand, functional reach, and Tinetti balance measures in community-dwelling older people. J Am Geriatr Soc 2004;52:1343-8.

34. Mathias S, Nayak US, Isaacs B: Balance in elderly patients: the "get-up and go" test. Arch Phys Med Rehabil 1986;67:387-9.

35. Morris S, Morris ME, Iansek R: Reliability of measurements obtained with the Timed “Up \& Go" test in people with Parkinson disease. Phys Ther 2001;81:810-8.

36. Podsiadlo D, Richardson S: The timed "Up \& Go": a test of basic functional mobility for frail elderly persons. J Am Geriatr Soc 1991;39:142-8.

37. Steffen TM, Hacker TA, Mollinger L: Age- and gender-related test performance in community-dwelling elderly people: Six-Minute Walk Test, Berg Balance Scale, Timed Up \& Go Test, and gait speeds. Phys Ther 2002;82:128-37.

38. Carrera E, Michel P, Despland PA, et al.: Continuous assessment of electrical epileptic activity in acute stroke. Neurology 2006;67:99-104.

39. Cuspineda E, Machado C, Aubert E, et al.: Predicting outcome in acute stroke: a comparison between QEEG and the Canadian Neurological Scale. Clin Electroencephalogr 2003;34:1-4. 
40. Finnigan SP, Rose SE, Walsh M, et al.: Correlation of quantitative EEG in acute ischemic stroke with 30-day NIHSS score: comparison with diffusion and perfusion MRI. Stroke 2004; 35:899-903.

41. Pfurtscheller G, Brunner C, Schlogl A, et al.: Mu rhythm (de)synchronization and EEG single-trial classification of different motor imagery tasks. Neuroimage 2006;31:153-9.

42. Forss N, Silen T: Temporal organization of cerebral events: neuromagnetic studies of the sensorimotor system. Rev Neurol 2001;157:816-21.

43. Hewitt CE, Torgerson DJ: Is restricted randomisation necessary? Bmj 2006;332:1506-8.

44. Twisk J, de Vente W: Attrition in longitudinal studies. How to deal with missing data. J Clin Epidemiol 2002;55:329-37.

45. Thomas L, Cullum N, McColl E, et al.: Guidelines in professions allied to medicine. Cochrane Database Syst Rev 2000(2):CD000349.

46. Ramachandran VS, Oberman LM: Broken mirrors: a theory of autism. Sci Am 2006;295:62-9.

47. Crosbie JH, McDonough SM, Gilmore DH, Wiggam MI: The adjunctive role of mental practice in the rehabilitation of the upper limb after hemiplegic stroke: a pilot study. Clin Rehabil 2004;18:60-8.

48. Dickstein R, Dunsky A, Marcovitz E: Motor imagery for gait rehabilitation in post-stroke hemiparesis. Phys Ther 2004;84:1167-77.

49. Dijkerman HC, Letswaart M, Johnston M, MacWalter RS: Does motor imagery training improve hand function in chronic stroke patients? A pilot study. Clin Rehabil 2004;18:538-49.

50. Malouin F, Belleville S, Richards CL, et al.: Working memory and mental practice outcomes after stroke. Arch Phys Med Rehabil 2004;85:177-83.

51. Malouin F, Richards CL, Desrosiers J, Doyon J: Bilateral slowing of mentally simulated actions after stroke. Neuroreport 2004;15:1349-53.

52. Malouin F, Richards CL, Doyon J, et al.: Training mobility tasks after stroke with combined mental and physical practice: a feasibility study. Neurorehabil Neural Repair 2004;18:66-75.

53. Page SJ: Imagery improves upper extremity motor functions in chronic stroke patients with hemiplegia: a pilot study. Occup Ther J Res 2000;20:200-15.

54. Page SJ, Levine P, Leonard AC: Effects of mental practice on affected limb use and function in chronic stroke. Arch Phys Med Rehabil 2005;86:399-402.

55. Page SJ, Levine P, Sisto SA, Johnston MV: Mental practice combined with physical practice for upper-limb motor deficit in subacute stroke. Phys Ther 2001;81:1455-62.

56. Page SJ, Levine P, Sisto S, Johnston MV: A randomized efficacy and feasibility study of imagery in acute stroke. Clin Rehabil 2001;15:233-40. 


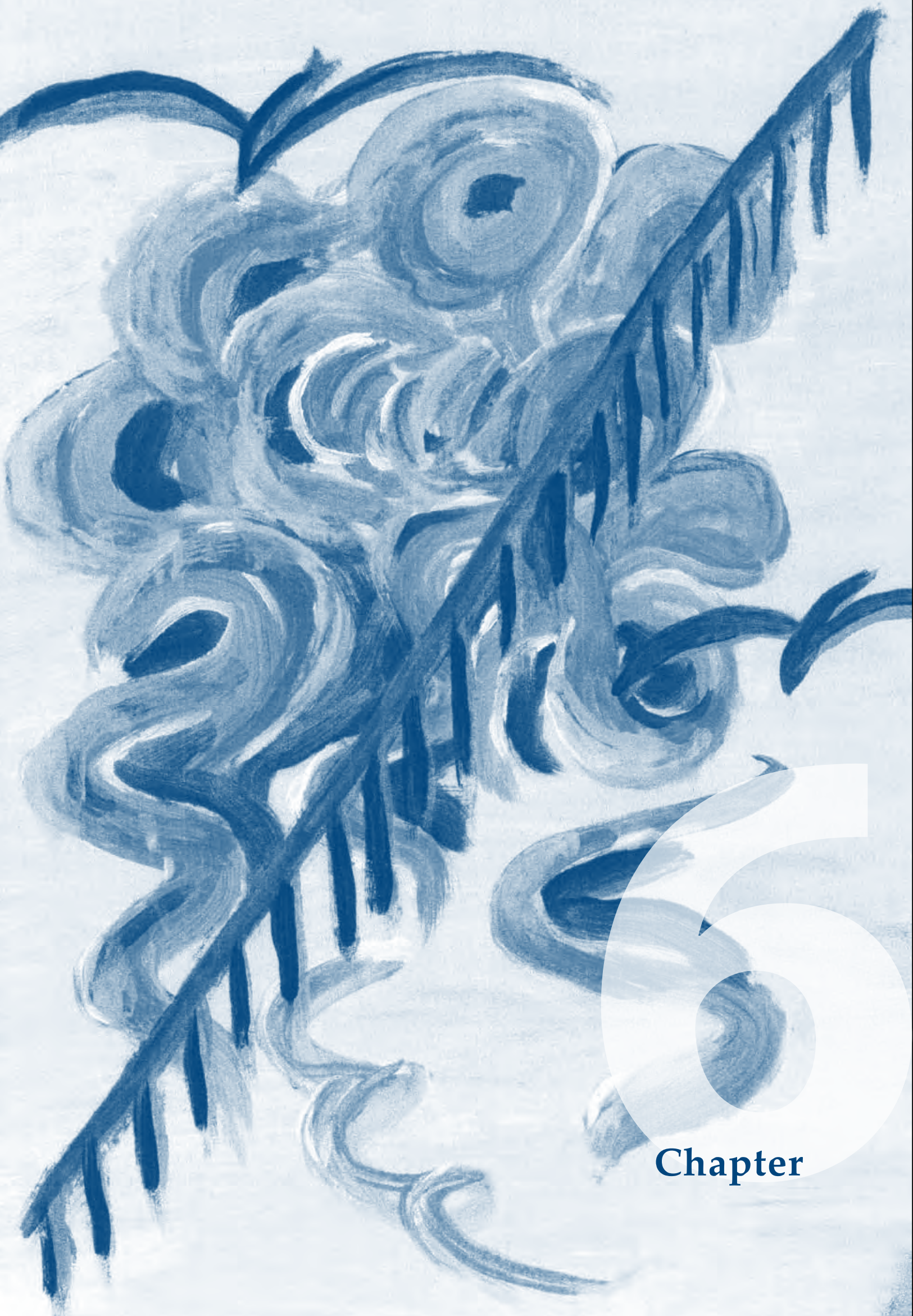




\section{Chapter six}

Feasibility of a mental practice intervention in stroke patient in Dutch nursing homes a process evaluation

Braun SM, van Haastregt JC, Beurskens AJ, Gielen AI, Wade DT, Schols JM.

(Submitted 2009) 


\section{Abstract}

Background: Within a multi-centre randomised controlled trial in three nursing homes, a process evaluation of a mental practice intervention was conducted. The main aim was to determine if the intervention was performed according to the framework and to describe the therapists' and participants' experiences with the intervention.

Methods: The six week mental practice intervention was given by physiotherapists and occupational therapists in the rehabilitation teams and consisted of four phases: explanation of imagery, teaching patients how to use imagery, using imagery as part of therapy, and facilitating the patient in using it alone and for new tasks. It had a mandatory and an optional part. Data were collected by means of registration forms, pre structured patient files, patient logs, selfadministered questionnaires, and a focus group interview.

Results: A total of 14 therapists and 18 patients with stroke in the sub acute phase of recovery were involved. Response rates differed per assessment (range 57-100\%). Two patients dropped out of the study (total $n=16$ ). The mandatory part of the intervention was given to 11 of 16 patients: 13 received the prescribed amount of mental practice and 12 practiced unguided outside of therapy. The facilitating techniques of the optional part of the framework were partly used. Therapists were moderately positive about the use of imagery in this specific sample. Several barriers were reported. Although it was harder for some patients to generate images than others, all patients were positive about the intervention and reported perceived short term benefits from mental practice.

Discussion: Although the majority of the sample was given mental practice according to the framework, the intervention was less feasible than we hoped. Implementing a complex therapy delivered by existing multi-professional teams to a vulnerable population with a complex pathology poses many challenges. 


\section{Feasibility of a mental practice intervention in stroke patients in Dutch nursing homes: a process evaluation}

\section{Background}

Stroke is one of the leading causes of death and disabilities globally (1). Treating patients after stroke is challenging: patients are often very vulnerable, especially in the sub acute phase of recovery, and stroke is a complex pathology which can lead to a variety of symptoms. Much research on effectiveness of treatments within stroke has been performed, but there is no evidence supporting most specific rehabilitation treatments (2). Only a small percentage of the day in organized stroke units is spent with therapists (3), which may not be optimal as a higher amount of practice is known to be related to more improvement $(4,5)$.

Mental practice of tasks is a relatively new therapy that is receiving increasing attention within rehabilitation research. Practising a skill mentally is potentially a method to increase the amount of therapy during rehabilitation in a safe way with relatively low costs. Mental practice has been defined in many ways, and our definition (6) used for this study was a training or therapy form in which an internal representation of the movement is activated and the execution of the movement repeatedly mentally simulated, without physical activity, within a chosen context. There is some evidence that mental practice might be effective in patients with stroke (6-8).

In the Netherlands, the majority of stroke healthcare takes place in nursing homes in which patients are treated by multidisciplinary teams according to stroke guidelines $(1,9,10)$, but the younger and less cognitively impaired minority of patients are admitted to stroke rehabilitation centres. In a randomized controlled trial, we assessed the effects of mental practice on recovery of stroke patients in three Dutch nursing homes. The mental practice framework developed (11) consisted of a mandatory and an optional part. The intervention was given by physiotherapists and occupational therapists.

As part of this randomized controlled trial we performed a detailed process evaluation to determine what actually happened when the 'new' treatment was given: this study may help improve the feasibility of mental practice for therapists and patients. The aims of this study were to evaluate (1) which aspects of the mental practice intervention were delivered according to the framework, and to what extent, and (2) the therapists' and participants' experiences with the intervention. 


\section{Method}

\section{Study design and population}

This process evaluation is a descriptive study in which both quantitative and qualitative data were gathered. The study was approved by the local medical ethical committee (Atrium MC, HsZuyd, Orbis medical concern, internal number 07-T-18). The study sample consisted of all the patients with stroke from three Dutch nursing homes allocated to the mental practice group of the trial.

Eight physiotherapists and six occupational therapists performed the intervention. All therapists conducting the intervention were already specialized in stroke rehabilitation and most had some awareness of mental practice but did not use it regularly. Details about the design of the randomized controlled trial are presented elsewhere ((12), www.trialregister.nl I NTR993).

\section{Intervention}

We developed a mental practice framework which allowed therapists and patients to tailor the intervention to the patients needs (11). A flexible protocol was used because rehabilitation needs to be tailored to the preferences and abilities of each patient seen, especially when patients have complex problems such as seen in people after stroke. This study was set in the context of regular clinical (non-research) rehabilitation practice, and so the framework was developed to be embedded in regular therapy sessions of 30 minutes where the actual allocation of time to specific aspects of therapy necessarily varied according to the patient's needs and wishes. This study aimed to investigate what actually happened.

The framework has been described in detail (11) and it has four phases: (1) explanation of imagery, (2) teaching patients how to use imagery, (3) using imagery as part of therapy, and (4) facilitating the patient in using it alone and for new tasks.

Explanation. Mental practice was explained to the patient by the therapist: what it is, how it might help and what it cannot do (to avoid unrealistic expectations). In addition, skills which the patients wanted to improve were chosen with the patient. Apart from 'drinking' and 'walking', which were the same for all patients, patients could choose two other skills with their occupational therapist and physiotherapist: one for the arms and one for the legs.

Teaching. In this phase patients were first taught to imagine the sequences of movement needed to achieve the task set, with an emphasis upon achieving the correct sequence. Then they were taught how to maximize the vividness (reality) of the imagined experience. Teaching involved 
alternation of physical and mental practice, using the physical practice to generate a correctly sequenced, vivid mental representation. The balance varied according to a patient's needs. Each patient could choose the representation (first or third person) that they found easiest (13-15). In the original description (11), nine optional facilitating techniques were given to be used to assist this training.

The success of a patient in imagining the actions correctly and vividly was judged by self-report, checked as far as possible by comparing the time taken to perform a task mentally against the time in reality, and by checking that the patient could recite the order of actions correctly.

Use in practice. Once the therapist judged that the patient had learned how to practice mentally, it was incorporated into therapy. Patients were asked to use it primarily before attempting the task concerned (outside therapy sessions as well). Furthermore as patients reported that imagining the separate actions was becoming easier, they were encouraged to practice groups of actions and eventually the whole task as a single unit, so-called chunking (16).

Self-directed use. As patients gained confidence, needing less support and reassurance, the therapist reduced the frequency and extent of the support offered. They also encouraged the patient to develop its use for other tasks.

The mental practice intervention was given for six weeks and consisted of a mandatory and an optional part.

\section{The mandatory part}

- After mental practice was explained (phase 1), the patients were supposed to receive at least ten mental practice training sessions embedded in regular therapy spread over at least three weeks. These sessions were to be guided by a trained therapist.

- As soon as the therapists thought patients were able to perform imagery outside of therapy they were supposed to encourage unguided mental practice at once. When the therapist thought patients were able to practice correctly outside of therapy, logs (one per week) had to be handed to the patients to record unguided mental practice behavior. In principle, a maximum of six logs could be filled in.

\section{The optional part}

In the framework (11) nine facilitating techniques to teach imagery were given: talking through or verbalizing the movement by either the patient [1] or the therapist [2]; [3] enhancing sensory information by passively moving a body limb or joint; [4] giving visual cues by watching clips of 
movements from a DVD; [5] observing oneself in the mirror; [6] observing others (including demonstration by the therapist); [7] using audio cues; [8] using visual cues; and, [9] extracting a part of a movement from the entire skill to practice mentally (e.g. placing the foot within a step) before embedding the part back into the entire skill. Choices for specific facilitating techniques were based on shared decisions.

\section{Training of therapists}

In each nursing home, therapists were trained in teaching patients about mental practice through a presentation of the theoretical background, two half day workshops, practical work with patients and group discussions. The therapists received written instruction (course map, selected background literature) and additional materials to facilitate performing mental practice (e.g. patient logs, pre structured patients files, video clips of daily movements). A group meeting with therapists from all three nursing homes was held shortly after starting to discuss differences and difficulties in application of the framework. Therapists could always call for additional help of the researcher (SB) during the trial. Both physiotherapists and occupational therapists worked according to the framework, but often they were working on different tasks such as using stairs and feeding with a spoon.

\section{Data collection}

Data from the therapists were collected by means of registration forms and pre structured patient files in which therapists recorded the therapy frequency and content and also clinical progress of the patient. At the end of the intervention period the therapists filled out a questionnaire, and a focus group interview was undertaken, asking about their experiences and opinions (AG). Representatives of each house were invited, with a maximum of three per location. Therapists could decide who would attend. An independent interviewer, experienced in performing focus group interviews, lead the interview which was audio taped.

Data from the patients were collected by means of patient logs and a self-administered questionnaire. Patients were asked to keep a log of the use of mental practice outside of therapy, and to record their mood and anything else of note during the intervention period (appendix). Patients were handed a self-administered questionnaire after their six weeks of treatment.

\section{Data analysis}

Data from the pre structured patients' files and logs were extracted by a researcher (SB).

Quantitative data from the questionnaires were analysed and put into frequency tables and open comments were clustered in themes. 
The focus group audio tape was transcribed verbatim by an independent rater (AG). First, the interviews were read in total to get a sense of the whole data before breaking them into parts. Remarks were placed in the margin and redundant information (e.g. interruptions, small talk between therapists) was removed in the initial process of exploring the data. Two independent researchers determined the main topics (mainly the topics from the interview guide) and compared results. From the transcript an anonymous summary was made, illustrated by quotations. The interview report was send to all participants with the request to check the report on correctness. Comments were processed and quotes used to illustrate the main results.

\section{Results}

The study sample consisted of five male and 13 female patients with an average age of 77.7 years (SD 7.2 years). Patients were included between the second and 10th week post-stroke with the average being six weeks (SD 2.7 weeks) post stroke at the beginning of the intervention period. During the intervention period two of the 18 patients dropped out: one of these two perceived that mental practice was not of benefit to him/ her: the other left for reasons unrelated to the intervention.

Of the 14 therapists taught, one physiotherapist did not participate in the entire trial because he started working elsewhere and one occupational therapist missed a part because of maternity leave. The response rates of the different measurement instruments are presented in table 1 (next page).

\section{Performance according to framework: the mandatory part}

\section{Therapy amount}

The 16 remaining patients generally attended therapy. In three pre structured patient files a short illness period due to influenza was reported, interrupting the intervention for two days in phase two $(n=2)$ or phase $3(n=1)$. Occasionally a therapy session was cancelled because of a doctor's appointment at the hospital, but the amount of therapy 'lost' was small. The number of treatments given to an individual patient ranged from 10 to 48 therapy sessions, with an average of 24 (SD 9.8) sessions in six weeks.

In one nursing home the data on the amount of mental practice treatment was lost because therapists partly used their own patient files: this affected the registered amount of treatment per step of the framework in two patients. 
Tab. 1: Overview of response groups, group sizes and response rates for the different measures.

\begin{tabular}{|c|c|c|c|}
\hline Measure & $\begin{array}{l}\text { Attending, } \\
\text { completed or } \\
\text { received }\end{array}$ & $\begin{array}{l}\text { Attending rate, } \\
\text { Response rate }\end{array}$ & Comments \\
\hline $\begin{array}{l}\text { Pre structured } \\
\text { patient files } \\
\text { Patients } \\
\text { treated: } 16\end{array}$ & $\begin{array}{l}\text { Patient files } \\
(\mathrm{n}=14)\end{array}$ & $\begin{array}{l}\text { Response rate: } \\
88 \%\end{array}$ & $\begin{array}{l}\text { Two patients dropped out before ending of six } \\
\text { week intervention, leaving sixteen patients } \\
\text { treated. In another two patients the pre } \\
\text { structured registration form was not used. } \\
\text { fourteen patient files were therefore checked. }\end{array}$ \\
\hline Patient logs & $\begin{array}{l}\text { Filled in } \\
\text { correctly: } 58\end{array}$ & $\begin{array}{l}\text { Response rate: } \\
75 \%\end{array}$ & $\begin{array}{l}\text { twelve out of sixteen patients used a log for one } \\
\text { or more weeks, at average four logs per patient } \\
\text { during six weeks. }\end{array}$ \\
\hline $\begin{array}{l}\text { Questionnaire } \\
\text { therapists at } \\
\text { end of the } \\
\text { trial } \\
\text { Handed: } 14\end{array}$ & $\begin{array}{l}\text { Completed } \\
(\mathrm{n}=8) \text {, but } 12 \\
\text { therapists } \\
\text { contributed }\end{array}$ & $\begin{array}{l}\text { Response rates: } \\
\text { Therapists: } \\
86 \% \text {, } \\
\text { Questionnaires: } \\
57 \%\end{array}$ & $\begin{array}{l}\text { One questionnaire was filled in by two therapists, } \\
\text { and another by four therapists }\end{array}$ \\
\hline $\begin{array}{l}\text { Questionnaire } \\
\text { patients after } \\
\text { six week } \\
\text { intervention } \\
\text { Handed: } 15\end{array}$ & $\begin{array}{l}\text { Completed } \\
(\mathrm{n}=14)\end{array}$ & $\begin{array}{l}\text { Response rate: } \\
93 \%\end{array}$ & $\begin{array}{l}\text { One patient did not receive a questionnaire } \\
\text { (forgotten). Of the fifteen handed questionnaires, } \\
\text { one was not completed. }\end{array}$ \\
\hline $\begin{array}{l}\text { Focus group } \\
\text { interview at } \\
\text { end of the } \\
\text { trial } \\
\text { Invited: } 9\end{array}$ & $\begin{array}{l}\text { Therapists } \\
\text { attending }(n=7)\end{array}$ & $\begin{array}{l}\text { Attending rate: } \\
78 \%\end{array}$ & $\begin{array}{l}\text { One therapist had left the nursing home and one } \\
\text { therapist was on maternity leave. Therapists from } \\
\text { all locations were present. }\end{array}$ \\
\hline
\end{tabular}

Thirteen (93\%) out of the 14 patients with full information received the obligatory amount of at least 10 sessions of mental practice (phase 2): three participants completed the entire framework, eight were in step three after six weeks and step one and two were achieved in three patients. One patient did not receive the required amount of practice therapy as determined in the mandatory part. It is notable that the last step of the framework was rarely reached, but the focus group data revealed that some therapists did not know exactly when they should move on to a next step.

The main reasons given for not completing all four steps of the framework were that therapists thought that patients did not understand what they were supposed to do, were unwilling to perform imagery or got frustrated by 'thinking too much'.

\section{Unguided mental practice}

Twelve out of the sixteen patients (75\%) practiced unguided outside of therapy during the six week intervention period (tab. 2). Four participants did not return any of the logs or did not record practising mentally outside of guided therapy. Among these four participants were also the two patients of whom no data on the number of treatments were recorded. 
On average the remaining 12 participants (75\%) filled in five logs (representing mental practice over five weeks), with a range from one to six logs completed. The records showed that these 12 participants at average practiced 635 minutes (SD 522 minutes) during 55 unguided sessions (SD 45 sessions). One unguided mental practice session could be between two and 30 minutes long. The minimum time over six weeks spent on mental practice outside of therapy was one hour and 10 minutes and the maximum was 29 hours and 11 minutes.

\section{Performance according to the framework: the optional part}

The optional facilitating techniques most frequently used to teach imagery were 'talking through or verbalizing the movement by the patient' (51\%) and 'talking through and verbalizing movement by therapist' (38\%). Occasionally, visual cues from the surroundings (4\%), 'extracting a part of a movement from the entire skill' (4\%), 'observing oneself in the mirror' $(2 \%)$ and 'observing other patients' (1\%) were reported. Other techniques were not or rarely used (tab. 2).

Tab. 2: The most frequently used facilitating techniques by the therapists in the trial to teach and monitor imagery use. The number of logs used by the individual patients and number of days practiced outside of therapy during the six weeks of the intervention. The amount of sessions of unguided therapy and the amount of time spend on mental practice outside of therapy, recorded in minutes.

\begin{tabular}{|c|c|c|c|c|c|c|c|c|c|c|}
\hline \multirow[b]{2}{*}{$\begin{array}{l}\text { Home } \\
(\mathrm{A}, \mathrm{B}, \mathrm{C}) \\
\text { and } \\
\text { patient } \\
\text { number }\end{array}$} & \multicolumn{4}{|c|}{$\begin{array}{l}\text { Data on unguided imagery } \\
\text { (total over six weeks intervention time) }\end{array}$} & \multicolumn{6}{|c|}{$\begin{array}{l}\text { Facilitating techniques used (in \%) during } \\
\text { therapy sessions with therapists }\end{array}$} \\
\hline & $\begin{array}{l}\text { Amount } \\
\text { of logs } \\
(\max .6)\end{array}$ & $\begin{array}{l}\text { Amount } \\
\text { days } \\
\text { practiced } \\
\text { (max. } \\
42 \text { ) }\end{array}$ & $\begin{array}{l}\text { Number } \\
\text { mental } \\
\text { practice } \\
\text { sessions }\end{array}$ & $\begin{array}{l}\text { Total } \\
\text { amount } \\
\text { minutes } \\
\text { practiced }\end{array}$ & VP & VT & $\begin{array}{c}\text { VIC } \\
\text { demo }\end{array}$ & VEC & EXT & VICM \\
\hline A -101 & 6 & 35 & 95 & 683 & 67 & 21 & 8 & 0 & 0 & 0 \\
\hline A -105 & 1 & 4 & 10 & 100 & 0 & 14 & 6 & 6 & 0 & 0 \\
\hline A -106 & 6 & 23 & 23 & 940 & 42 & 16 & 0 & 0 & 0 & 0 \\
\hline A -109 & 6 & 33 & 136 & 1751 & 67 & 15 & 0 & 0 & 0 & 0 \\
\hline A -110 & 0 & 0 & 0 & 0 & 11 & 15 & 0 & 0 & 0 & 0 \\
\hline A -113 & 6 & 37 & 49 & 284 & 90 & 80 & 0 & 0 & 0 & 0 \\
\hline A -114 & 5 & 25 & 47 & 530 & 48 & 42 & 0 & 8 & 0 & 0 \\
\hline A -118 & 4 & 18 & 47 & 820 & 40 & 40 & 0 & 30 & 0 & 0 \\
\hline В -201 & 6 & 41 & 121 & 1098 & 51 & 3 & 0 & 0 & 0 & 0 \\
\hline B -206 & 0 & 0 & 0 & 0 & 28 & 0 & 0 & 0 & 39 & 0 \\
\hline B - 207 & 6 & 37 & 94 & 1060 & 32 & 0 & 0 & 0 & 0 & 0 \\
\hline B - 210 & 3 & 10 & 14 & 120 & 47 & 0 & 0 & 0 & 0 & 0 \\
\hline B - 211 & 5 & 17 & 20 & 162 & 0 & 63 & 0 & 0 & 0 & 0 \\
\hline C - 302 & 0 & 0 & 0 & 0 & ? & ? & ? & ? & $?$ & ? \\
\hline C -304 & 0 & 0 & 0 & 0 & ? & ? & ? & ? & $?$ & ? \\
\hline C -306 & 4 & 5 & 7 & 70 & 33 & 100 & 0 & 0 & 0 & 17 \\
\hline Total & 58 & 285 & 663 & 7618 & 556 & 409 & 14 & 44 & 39 & 17 \\
\hline Average & 4,8 & 23,75 & 55,23 & 634,8 & $51 \%$ & $38 \%$ & $1 \%$ & $4 \%$ & $4 \%$ & $2 \%$ \\
\hline
\end{tabular}

$V P=$ verbalizing movement patient $\mid V T=$ verbalizing movement therapist $\mid$ VICdemo $=$ visual internal cues through demonstration $\mid V E C=$ visual external cues $\mid$ EXT $=$ extracting parts of movement $\mid V I C M=$ visual internal cues through mirror observation $\mid ?=$ no data recorded 


\section{Therapists' experiences with the mental practice framework}

The 12 therapists involved throughout all reported (100\%) that the intervention was clear and if any problems occurred they had contacted the main researcher (SB) for assistance, which happened four times during the trial period. However, they admitted to gaining confidence with experience: 'When you however work longer with it or when you have had more patients, the framework is clear to work with'.

Ten therapists (86\%) considered themselves at least sufficiently capable of giving the mental practice intervention ('sufficient', $n=8$; 'good', $n=2$ ): two did not answer this question.

Three therapists from the same location reported that they could implement mental practice within therapy time as planned. Seven therapists needed between 10 and 20 additional minutes per therapy session and another two therapists were unable to estimate how much longer they needed.

In the focus group and written comments, therapists reported that it was hard to check compliance of the patient. This may have lead to longer instruction times and seemed often to lead to a feeling of irritation (for the therapist). '... but you just cannot check if they have practiced, which is very annoying'. Another therapist was more optimistic: 'I believe that you do know who has practiced (the 'good' ones) and who has not (the 'bad' ones). However, there are a lot of patients that remain in a grey area in which you just don't know what they have done'. Another therapist added: 'It is still something completely subjective, you cannot touch it, it is not measurable. You cannot check thoughts'.

Therapists were not sure whether the patients correctly registered the unguided mental training in the logs: 'Sometimes they would report what they had practiced physically outside of therapy or during therapy. You really do not know for sure if the reported practice is about mental practice and if it is correct or not'.

However, all therapists thought that the patient logs were of some use to record unguided imagery despite doubts on the validity of the reported information.

\section{Therapists' opinion on benefits and use after the trial}

Table 3 (next page) shows the opinions of therapists about benefits reported in the questionnaires. During the focus group interview all therapists mentioned an increase in patients' motivation and autonomy to work on recovery because of mental practice: 'I believe that patients are more motivated, they are more consciously involved and that way we might give a piece of self esteem back to the patient.

They feel they are not dependent on the therapist and can work on recovery themselves'. Another therapist added: '... and more self confidence. You give them something that they cannot do physically. For instance, when the patient is standing between the parallel bars and is not yet capable of walking yet, then they can 
Tab. 3: Possible benefits stroke patients might perceive from an additional mental practice intervention. Eleven therapists filled in the questions on possible benefits and twelve the last three questions.

\begin{tabular}{|c|c|c|c|}
\hline & Yes, most patients & Yes, some patients & $\begin{array}{l}\text { No, none of the } \\
\text { patients }\end{array}$ \\
\hline Improve movements & 2 & 9 & - \\
\hline Less anxious & 1 & 5 & 5 \\
\hline Secure feeling & 1 & 10 & - \\
\hline More self confidence & 5 & 6 & - \\
\hline Motivate for therapy & 1 & 10 & - \\
\hline Strategy training & 4 & 6 & 1 \\
\hline Other reason & - & - & - \\
\hline Are patients able to image? & - (Yes) & 11 (Don't know) & - (No) \\
\hline MP beneficial to recovery & 2 & 10 & - \\
\hline MP part of guidelines & 1 & 9 & 2 \\
\hline Apply MP after trial & 7 & 5 & - \\
\hline$\%$ population feasible & $10-25 \%$ & & \\
\hline
\end{tabular}

$M P=$ mental practice

practice this mentally. Often there is, especially at the beginning, fear of falling, fear of not succeeding, a hesitation that they are not able to walk. But by practising mentally, you decrease fear and gain self confidence'.

Seven therapists reported they would use mental practice after the trial. 'You use it as one of the many tools a therapist has. With one patient you might use imagery in another you might not'. However, four therapists doubted if they would use the entire framework: 'I don't believe I would go through the entire four steps of the framework. I would extract pieces from it, some things I will use, others not'. Four therapists were not sure if they were going to use mental practice.

\section{Patients' experiences with the mental practice framework}

Patients reported several ways that they were facilitated to learn and use imagery. Four participants reported through the questionnaire that observing the movement first in others was the most helpful way to learn to use imagery. Observing could then be followed by practising together, observing others or breaking the movement up into small parts. Another four patients thought that 'talking through and verbalizing the movement' was the most effective way to generate images. Other ways patients preferred to learn were: 'continuously repeating' and 'experiencing 
support from the therapists' (these were not mentioned in the nine facilitating techniques). Three participants could not remember what helped most and one person did not answer this question (tab. 4).

\section{Patients' opinion on short term perceived benefits and mental practice use}

At the end of the six week intervention period, patients were asked if they had perceived benefits from the mental practice framework. Seven participants responded they benefited a lot from the intervention and the remaining seven patients reported that they had perceived some benefit from mental practice. The perception of benefits could not be related to the effort a participant had to make in order to generate images. Only three patients said it was easy to practice mentally, six participants were neutral and five patients found it hard to generate images. Although all patients perceived some level of benefit only seven were sure they would continue to practice mentally after the intervention period, four did not know yet en three said they would not continue, of which one eventually did practice during the follow up period. The perceived benefits were general, and concerned preparing for movement and gaining confidence (tab. 4, next page).

\section{Discussion}

The majority (eleven out of sixteen, 69\%) of the participants in this study were treated according to the framework: they received the minimum amount of mental practice (thirteen out of fourteen, 93\%), and they undertook unguided (twelve out of sixteen, 75\%) practice.

Nonetheless, applying a mental practice framework in stroke patients in the sub-acute phase of recovery was much harder than expected, and teaching and monitoring mental practice may need a longer and more intensive training period than given in this study. Whether this additional effort is compensated for later as the patient continues unguided mental practice is uncertain. Some patients do not practice outside of therapy at all and in these patients the advantage of teaching mental practice will most likely be lost (13).

When we developed the mental practice intervention we deliberately allowed therapists to tailor the intervention to the needs and abilities of the individual patient. This flexibility was considered important but also seemed to make it more difficult and confusing for the therapists. Although therapists were content about the training of the mental practice framework and knew what was expected, once they tried to use it they would have liked to have had more certainties in order to know if they were treating patients 'correctly'. Moreover, the variation between therapists in the extent to which they used the protocol or supported mental practice suggests that it may have been hard to alter professional routines during the trial. This seemed more the case for the participating physiotherapists than for the occupational therapists. 
Tab. 4: Reports of patients at the end of the six weeks intervention period with mental practice (T1) about perceived benefits and use of mental practice. Which skills were rehearsed mentally and comments are reported in the last two columns.

\begin{tabular}{|c|c|c|c|c|c|c|}
\hline & $\begin{array}{c}\text { Perceived } \\
\text { benefits }\end{array}$ & $\begin{array}{l}\text { Effort to } \\
\text { perform } \\
\text { imagery }\end{array}$ & $\begin{array}{l}\text { Skills MP } \\
\text { helped }\end{array}$ & $\begin{array}{l}\text { Skills MP } \\
\text { did not } \\
\text { help }\end{array}$ & $\begin{array}{l}\text { Intending } \\
\text { to use it } \\
\text { after } 6 \\
\text { weeks }\end{array}$ & $\begin{array}{l}\text { Facilitators for } \\
\text { learning and } \\
\text { using imagery }\end{array}$ \\
\hline A-109 & A lot & Easy & $\begin{array}{l}\text { Arm } \\
\text { movements, } \\
\text { right foot/leg }\end{array}$ & $\begin{array}{l}\mathrm{N} / \mathrm{A} \\
\text { It always } \\
\text { helped }\end{array}$ & Yes & $\begin{array}{l}\text { When the therapists } \\
\text { verbalizes the } \\
\text { movement during } \\
\text { performance of } \\
\text { the skill (not } \\
\text { demonstrating } \\
\text { or verbalizing self) }\end{array}$ \\
\hline B-210 & A lot & Easy & $\begin{array}{l}\text { Walking stairs } \\
\text { and getting up } \\
\text { from the floor }\end{array}$ & $\mathrm{N} / \mathrm{A}$ & Yes & $\begin{array}{l}\text { Going through the } \\
\text { steps before the skill } \\
\text { performance, f.i. } \\
\text { getting up from the floor }\end{array}$ \\
\hline A-101 & A lot & Neutral & $\begin{array}{l}\text { Walking and } \\
\text { becoming } \\
\text { more secure }\end{array}$ & N/A & Yes & $\begin{array}{l}\text { By experiencing the } \\
\text { support and directions } \\
\text { of the therapists }\end{array}$ \\
\hline A-118 & A lot & Neutral & $\begin{array}{l}\text { Turning in } \\
\text { bed, getting } \\
\text { out of bed, } \\
\text { walking }\end{array}$ & $\begin{array}{l}\text { Washing } \\
\text { and getting } \\
\text { dressed }\end{array}$ & Yes & $\begin{array}{l}\text { Demonstrations of the } \\
\text { correct movement, } \\
\text { observing others and } \\
\text { chopping the movement } \\
\text { up in small peaces }\end{array}$ \\
\hline B-211 & A lot & Neutral & $\begin{array}{l}\text { Standing up } \\
\text { from a chair }\end{array}$ & $\begin{array}{l}\text { Keeping } \\
\text { my balance }\end{array}$ & $\begin{array}{l}\text { Not sure } \\
\text { (not up to } \\
\text { it yet) }\end{array}$ & $\begin{array}{l}\text { Demonstrations and } \\
\text { practising together with } \\
\text { the therapist }\end{array}$ \\
\hline C-304 & A lot & Hard & Walking & $\begin{array}{l}\text { Did not } \\
\text { use it for } \\
\text { anything } \\
\text { else but } \\
\text { walking }\end{array}$ & No & $\begin{array}{l}\text { I cannot remember which } \\
\text { exercises or instructions } \\
\text { facilitated use of MP most }\end{array}$ \\
\hline C-306 & A lot & Hard & $\begin{array}{l}\text { Standing up } \\
\text { from a chair } \\
\text { and walking }\end{array}$ & $\mathrm{N} / \mathrm{A}$ & No & $\begin{array}{l}\text { I cannot remember which } \\
\text { exercises or instructions } \\
\text { facilitated use of MP most }\end{array}$ \\
\hline A-114 & Some & Easy & $\begin{array}{l}\text { Walking with } \\
\text { cane }\end{array}$ & $\mathrm{N} / \mathrm{A}$ & Not sure & $\mathrm{N} / \mathrm{A}$ \\
\hline A-105 & Some & Neutral & $\begin{array}{l}\text { Walking, } \\
\text { talking and } \\
\text { putting on } \\
\text { t-shirt }\end{array}$ & $\begin{array}{l}\text { Talking: } \\
\text { making } \\
\text { S-sound }\end{array}$ & Yes & $\begin{array}{l}\text { When the therapist } \\
\text { explains the separate } \\
\text { performance steps, lets } \\
\text { me repeat them a lout and } \\
\text { then lets me image the } \\
\text { movement in my mind. }\end{array}$ \\
\hline A-110 & Some & Neutral & $\begin{array}{l}\text { Standing up } \\
\text { from a chair, } \\
\text { walking with } \\
\text { aids }\end{array}$ & $\begin{array}{l}\text { N/A } \\
\text { No idea }\end{array}$ & Yes & $\begin{array}{l}\text { By chopping up the } \\
\text { movement in parts and } \\
\text { then repeat them } \\
\text { (accompanied by cues/ } \\
\text { words, like 'left' and } \\
\text { 'right') }\end{array}$ \\
\hline
\end{tabular}


Tab. 4: Reports of patients at the end of the six weeks intervention period with mental practice (T1) about perceived benefits and use of mental practice. Which skills were rehearsed mentally and comments are reported in the last two columns. (Continued)

\begin{tabular}{|c|c|c|c|c|c|c|}
\hline & $\begin{array}{l}\text { Perceived } \\
\text { benefits }\end{array}$ & $\begin{array}{l}\text { Effort to } \\
\text { perform } \\
\text { imagery }\end{array}$ & $\begin{array}{c}\text { Skills MP } \\
\text { helped }\end{array}$ & $\begin{array}{l}\text { Skills MP } \\
\text { did not } \\
\text { help }\end{array}$ & $\begin{array}{c}\text { Intending } \\
\text { to use it } \\
\text { after } 6 \\
\text { weeks }\end{array}$ & $\begin{array}{l}\text { Facilitators for } \\
\text { learning and } \\
\text { using imagery }\end{array}$ \\
\hline B-206 & Some & Neutral & Concentrating & $\mathrm{N} / \mathrm{A}$ & Not sure & $\begin{array}{l}\text { Demonstrations and } \\
\text { practising together with } \\
\text { the therapists }\end{array}$ \\
\hline B-207 & Some & Hard & $\begin{array}{l}\text { Standing up } \\
\text { from a chair }\end{array}$ & $\begin{array}{l}\text { With } \\
\text { walking, } \\
\text { but also } \\
\text { because of } \\
\text { bad knees }\end{array}$ & Not sure & Demonstrations \\
\hline B-106 & Some & Hard & $\begin{array}{l}\text { Driving with } \\
\text { wheel chair to } \\
\text { the table }\end{array}$ & $\begin{array}{l}\text { Transfer } \\
\text { wheel chair } \\
\text { to toilette }\end{array}$ & Yes & By continuously repeating \\
\hline C-302 & Some & Hard & $\begin{array}{l}\text { Supination } \\
\text { and pronation } \\
\text { of the arm }\end{array}$ & $\begin{array}{l}\text { N/A } \\
\text { Did not } \\
\text { use it for } \\
\text { any other } \\
\text { movements }\end{array}$ & No & $\begin{array}{l}\text { I cannot remember which } \\
\text { exercises or instruction } \\
\text { facilitated use of MP most }\end{array}$ \\
\hline
\end{tabular}

$N / A=$ not applicable $\mid M P=$ mental practice

The flexibility allowed arguably makes research difficult as there is less control over and know-ledge about the very specific details of treatments given. We tried to undermine this potential loss by asking the therapists to record as many as details as possible. Still, measuring different aspects of the mental practice intervention remained difficult. Our approach has been that the randomised trial was investigating the effect of a therapist teaching the patient about mental practice, and this study aimed to investigate (a) the teaching process and, as far as it is feasible (b) the direct effects of the teaching upon the patient's use of the technique.

On the other hand as shown in table 3, all therapists saw potential benefits from applying mental practice although only half intended to use it after the trial, and some only intended to use parts of the framework. The relatively long time needed to teach mental practice to patients may be due to reduced memory and concentration skills in our patients. In this context, it must be noted that Dutch nursing homes admit patients who are older, more frail and more cognitively impaired than those selected for specialist stroke rehabilitation centres. This may explain the lack of any effect seen in this study. Most other studies have been on younger and less frail patients. 


\section{Methodological quality of the study - limitations}

The limited number of participating patients and relatively many therapists supporting the trial led to a large number of therapists being involved in treating relatively few patients. Therefore, during this study the therapists had relatively few patients to gain familiarity with the techniques. The lack of routine might have influenced their answers in the questionnaire and interviews. For example, two therapists from two nursing homes and all the occupational therapists from one nursing home delivered only small amounts of mental practice therapy.

In general, it seemed as if the therapists were more positive about mental practice in the questionnaires, which were not anonymous, than in the focus group. The focus group was chaired by an independent interviewer (AG), unfamiliar to the therapists. Moreover the main focus of the interview was on barriers and problems in performing mental practice. Both these factors many have led to less positive answers in the focus group.

\section{Results compared to other literature}

Some detailed information on stroke patients' experience with imagery is given in one other study (17) where stroke patients were generally positive about applying mental practice.

Contrary to our mental practice framework, the intervention given is often a fixed program and training of therapists is minimal or not described (exception Liu and co-workers (18)).

Whether the interventions were given according to protocol was sometimes assessed.

Compliance of patients was in some studies checked with a $\log (19,20)$, an interview $(20)$, a phone call half way through (19) or self reported independent mental practice (18). All interventions seemed feasible, even the more complex one. We are unaware of any other detailed process evaluation of mental practice interventions in a clinical stroke trial.

We could not find any published data on the satisfaction of therapists with a mental practice intervention. Two studies reported that patients were satisfied with the imagery intervention and that no problems with the rehearsals or generalizations occurred $(18,20)$. How this satisfaction exactly was established is not clear. If or how patients were instructed to use imagery is not or seldom described in published effect studies, with the exception of mental practice through audio taped cassettes (20).

In this study, there seemed to be a mismatch between the experiences of the patients and therapists with the mental practice intervention. Generally the patients seemed more enthusiastic. 
Also, patients had additional goals for using mental practice besides practising motor skills, like increasing motivation and self confidence. The use of mental practice for these aims should be researched in future studies.

No negative effects of mental practice have been reported in stroke trials nor in this study (but we did not ask), but have been published in trials in which mental practice is part of a intervention to reduce chronic limb pain (21). Therapists sometimes did stop mental practice for a couple of days if they thought patients were 'thinking too much' and got irritated.

\section{Conclusion}

The study suggests that it is possible to teach patients who have had a stroke within the last 6-10 weeks how to use mental practice, but therapists probably need more support and practice to become confident and skilled in teaching patients. Therapists may specifically need additional support in learning how to tailor the teaching protocol to the individual needs of each patient. The majority of the patients were enthusiastic about mental practice. More research is needed to develop ways of monitoring the use of mental practice by patients - how much and how well. However this study shows the importance of discovering what patients and therapists actually do and experience when complex therapies like mental practice are introduced (22). Lastly we suggest that in future research into new therapies, therapists should have sufficient time to gain adequate experience before starting to research any new therapy technique, although this will require significant extra research funding!

\section{Competing interest}

All authors declare, that they have no commercial or competing interest relevant to the subject of the manuscript.

\section{Author's contributions}

$\mathrm{SB}, \mathrm{JVH}, \mathrm{AB}$, JS and DW made substantial contributions to conception and design. SB, JVH and $A B$ developed the questionnaires. $S B$ and $A G$ prepared and carried out the focus group interview. SB analyzed all pre-structured patient files and logs. All authors read and approved the final manuscript. 


\section{Acknowledgments}

We thank all interviewed therapists and all interviewed patients for sharing their thoughts, experiences and ideas with us in sometimes emotional times and difficult circumstances. We appreciate the help of Milou Brouns, Maartje Sangen, Bart Oudelaar and Marco Vandenboorn with the data collection. The study was funded by NutsOhra (SNO T-0702-70), VGZ (ZIP/HH/ ME/07.190) and the Mobiliteitsfonds (PR0601-B-E).

\section{References}

1. Commissie CVA-revalidatie. Revalidatie na een beroerte, richtlijnen en aanbevelingen voor zorgverleners. Den Haag: Nederlandse Hartstichting; 2001.

2. Langhorne P, Coupar F, Pollock A. Motor recovery after stroke: a systematic review. Lancet Neurol 2009;8:741-54.

3. Bernhardt J, Chan J, Nicola I, Collier JM. Little therapy, little physical activity: rehabilitation within the first 14 days of organized stroke unit care. J Rehabil Med 2007;39:43-8.

4. Kwakkel G, Kollen BJ, Wagenaar RC. Therapy impact on functional recovery in stroke rehabilitation: A critical review of the literature. Pysiotherapy 1999;85:377-91.

5. Kwakkel G, van Peppen R, Wagenaar RC, Wood Dauphinee S, Richards C, Ashburn A, et al. Effects of augmented exercise therapy time after stroke: a meta-analysis. Stroke 2004;35:2529-39.

6. Braun SM, Beurskens AJ, Borm PJ, Schack T, Wade DT. The effects of mental practice in stroke rehabilitation: a systematic review. Arch Phys Med Rehabil 2006;87:842-52.

7. Dickstein R, Deutsch JE. Motor imagery in physical therapist practice. Phys Ther 2007;87:942-53.

8. Sharma N, Pomeroy VM, Baron JC. Motor imagery: a backdoor to the motor system after stroke? Stroke 2006;37:1941-52.

9. van Peppen RP, Kwakkel G, Hermeling-van der Wel BC, et al. KNGF-richtlijn beroerte. Ned Tijdschr Fysiother 2004;114(Suppl.):1-77.

10. Schols JM, Crebolder HF, van Weel C. Nursing home and nursing home physician: the Dutch experience. J Am Med Dir Assoc 2004;5:207-12.

11. Braun SM, Kleynen M, Schols JM, Schack T, Beurskens AJ, Wade DT. Using mental practice in stroke rehabilitation; a framework. Clin Rehabil 2008;22:579-591.

12. Braun SM, Beurskens AJ, van Kroonenburgh SM, Demarteau J, Schols JM, Wade DT. Effects of mental practice embedded in daily therapy compared to therapy as usual in adult stroke patients in Dutch nursing homes: design of a randomised controlled trial. BMC Neurol 2007;15:34.

13. Jackson PL, Lafleur MF, Malouin F, Richards C, Doyon J. Potential role of mental practice using motor imagery in neurologic rehabilitation. Arch Phys Med Rehabil 2001;82:1133-41. 
14. Malouin F, Richards CL, Doyon J, Desrosiers J, Belleville S. Training mobility tasks after stroke with combined mental and physical practice: a feasibility study. Neurorehabil Neural Repair 2004;18:66-75.

15. Milton J, Small SL, Solodkin A. Imaging motor imagery: methodological issues related to expertise. Methods 2008;45:336-41.

16. Shumway-Cook A, Woollacott MH. Motor control: translating research into clinical practice. 3rd edition ed. Philadelphia: Lippincott Williams \& Wilkins; 2006.

17. Simmons L, Sharma N, Baron JC, Pomeroy VM. Motor imagery to enhance recovery after subcortical stroke: who might benefit, daily dose, and potential effects. Neurorehabil Neural Repair 2008;22:458-67.

18. Liu KP, Chan CC, Lee TM, Hui-Chan CW. Mental imagery for promoting relearning for people after stroke: a randomized controlled trial. Arch Phys Med Rehabil 2004;85:1403-8.

19. Dijkerman HC, Letswaart M, Johnston M, MacWalter RS. Does motor imagery training improve hand function in chronic stroke patients? A pilot study. Clin Rehabil 2004;18:538-49.

20. Page SJ, Levine P, Sisto S, Johnston MV. A randomized efficacy and feasibility study of imagery in acute stroke. Clin Rehabil 2001;15:233-40.

21. Moseley GL, Zalucki N, Birklein F, Marinus J, van Hilten JJ, Luomajoki H. Thinking about movement hurts: the effect of motor imagery on pain and swelling in people with chronic arm pain. Arthritis Rheum 2008;59:623-31.

22. Craig P, Dieppe P, Macintyre S, Michie S, Nazareth I, Petticrew M. Developing and evaluating complex interventions: the new Medical Research Council guidance. Bmj 2008;337:a1655. 


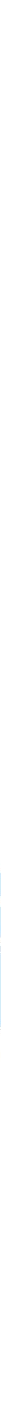




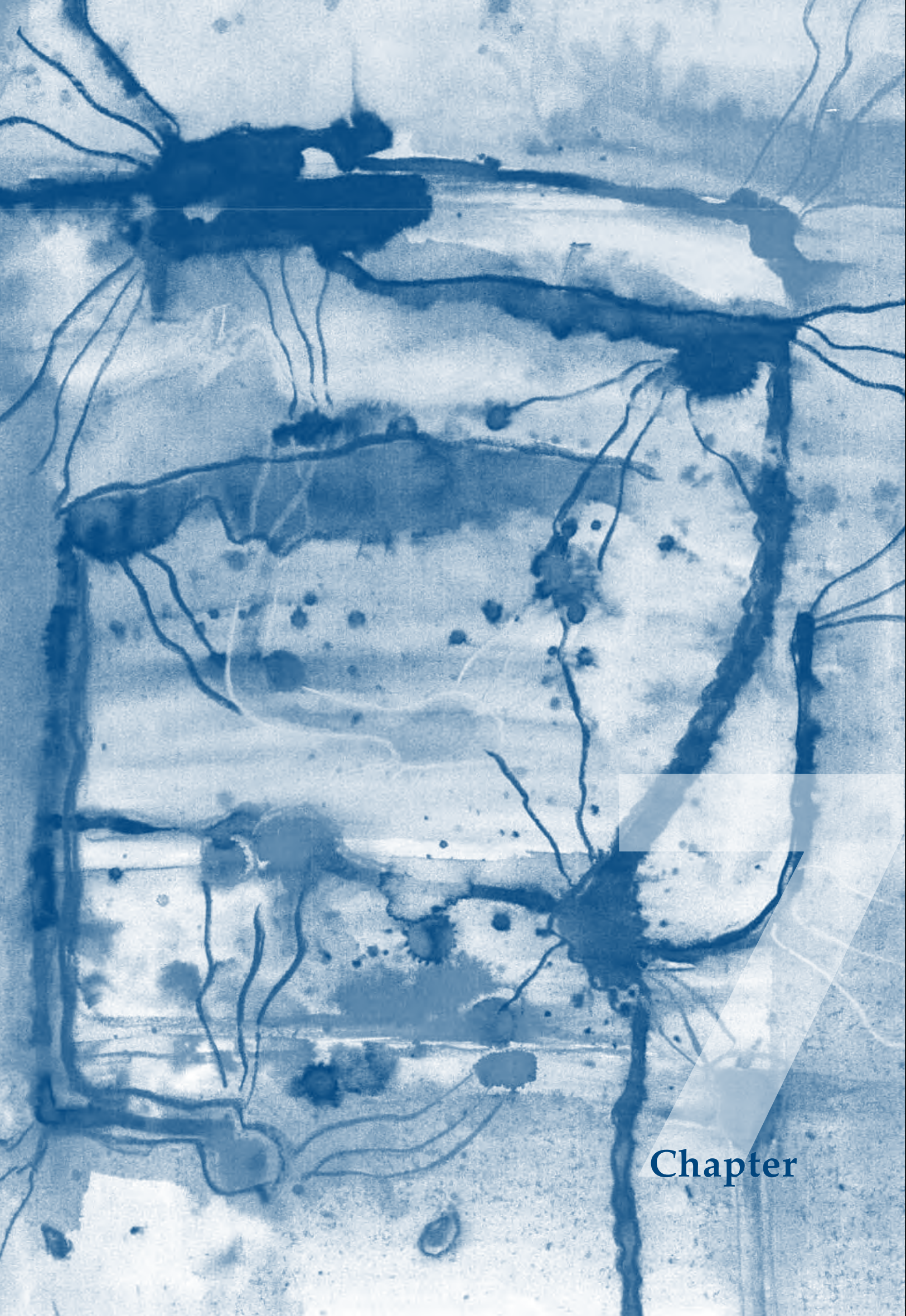




\section{Chapter seven}

Effects of mental practice in stroke rehabilitation in Dutch nursing homes: results from a randomised controlled trial

Braun SM, Beurskens AJ, Kleynen M, Oudelaar B, Schols JM, Wade DT.

(Submitted 2010) 


\section{Abstract}

Introduction: Systematic reviews suggest that mental practice as an additional therapy for people with stroke might be effective and suggest that more trials with better defined interventions are needed. This multi-centre randomised controlled trial investigated whether mental practice can contribute to a quicker and/or better recovery of stroke patients in the sub acute phase of recovery in Dutch nursing homes.

Methods: Over a six weeks intervention period both groups received multi professional therapy as usual. Additionally, patients in the experimental group had instruction on mental practice with a four step framework embedded in regular therapy time. Outcome was assessed at six weeks and six months. The primary outcome measure was the patient-perceived effect on performance of daily activities (10-point Likert scale). Six secondary outcomes on impairment and activity level were assessed. Primary analysis was performed according to the intention to treat principle. Generalized estimating equations (GEE) were used to analyse effects.

Results: Thirty-six adult stroke patients (average age 77,8 years, SD 7,2) were randomly assigned to the control or experimental group. No effect in favour of the mental practice intervention on any outcome measure could be detected at any of the measuring points.

Discussion: Stroke pathways in Dutch nursing homes select older patients which might have reduced the effects of training. The intervention given is complex for this population and outcome measures used may have contributed to the negative results. At present mental practice as described here cannot be recommended for this group of patients. 


\section{Effects of mental practice in stroke rehabilitation in Dutch nursing homes: results from a randomised controlled trial}

\section{Introduction}

In stroke, there is in general a relationship between the time spend on therapy and recovery: the more a patient practices, the better or faster he or she recovers (1). Movement imagery and mental practice have been put forward as an additional training method that might increase the amount of practice in a safe and cost efficient way (2), especially when therapy is limited by the physiological boundaries of the patient (e.g. fatigue) or insufficient therapist time (3).

Movement imagery is 'imagining oneself undertaking the skilled movement without actually doing the movement'. It primarily involves the person imagining movements. The subject may imagine the movement in the third (as if looking from a distance at oneself) or the first (as if looking through their own eyes) person's view (2).

When movement imagery is practiced on a regular basis it is called mental practice: during mental practice an internal representation of the movement is activated and the execution of the movement repeatedly mentally simulated within a chosen context. This mental simulation takes place in absence of bodily activity. It is used for the goal-oriented improvement or stabilization of a given movement (4). In this article, movement imagery is used when the technique of mentally rehearsing a skill is meant. When the emphasis is on the training aspect, using imagery as an additional training method as part of therapy, the term mental practice is used.

There is some evidence that mental practice might help to improve arm-hand function, foot sequence tasks, complex skills and mobility tasks in stroke patients $(4,5)$. However, the results from mental practice studies are hard to compare because there is a lack of consensus on how to teach, develop and monitor imagery. In addition, the conclusions from reviews could change by a relatively small number of new trials and little is known about the effectiveness of mental practice in routine clinical settings (6).

Therefore, a multi-centre randomised controlled trial was conducted over a two year period involving clinically diagnosed adult stroke patients in the sub acute phase of recovery. The overall aim of the proposed research project was to investigate the therapeutic potential of mental practice embedded in daily rehabilitation therapy on the improvement of daily activities of adult stroke patients compared to therapy as usual. 


\section{Methods}

Detailed information about this research protocol and the reasons underlying choices made are published elsewhere (7). The study protocol was approved by the local medical ethical committee and was conducted from October 2007 through December 2009 and patients included in the trial gave informed consent.

Decentralized randomisation took place by an independent third party blind for the patient's characteristics (8), based on a computerized (block size 4) randomisation schedule. No stratification took place. The randomisation procedure was the same for all three sites. Each patient recruited was registered and given the next sequential number within the nursing home, before the envelope was opened to determine their allocation. The third party then informed the independent rater (baseline measurements). Afterwards, the therapists were notified about which group the patient was assigned to.

Effects of the interventions were measured directly after the six weeks intervention period (T1) and six months after start of the intervention period (T2), by the same independent and blinded trained rater (MK).

The patients were not blinded to the treatment they received as they were aware of the treatment content. The rater however was blinded for the treatment allocation. A blinding check was performed after each of the two follow-up measurement sessions.

\section{Study population}

Stroke patients were recruited by the treating physicians or other staff and met the following inclusion criteria:

a) clinically diagnosed adult stroke patient, between two and 10 weeks after stroke onset;

b) sufficient cognitive level and communication skills to engage in mental practice, judged clinically for example by being able to follow simple instructions or perform a three stage command.

\section{Exclusion criteria:}

c) impairments prior to stroke causing persistent premorbid disability, such as rheumatic diseases or dementia.

\section{Treatment of participants}

All patients received six weeks of multi professional rehabilitation in accordance with the Dutch guidelines for stroke rehabilitation (9). Patients in the experimental group were additionally 
taught mental practice-techniques and principles to be embedded in every session. Paramedical therapists were instructed on how to treat the patients in the experimental group (Braun SM, van Haastregt JC, Beurskens AJ, Gielen X, Wade DT, Schols JM, submitted data, 2009). Patients allocated to the control group could be treated by any therapist.

\section{Experimental intervention}

Patients in the experimental group should not receive extra time for mental practice training with the therapist (i.e. teaching mental practice displaced other therapy activities). The mental practice intervention has been published elsewhere (10). The main points are that the intervention is a framework in which the physiotherapist and/or occupational therapist can tailor the imagery content to his own and the patients' abilities and preferences. Four steps are distinguished: explaining the concept (1), developing imagery techniques (2), applying mental practice (3) and consolidating (4). The protocol has a conditional and an optional part. To be included in the per protocol analysis, patients from the experimental branch should have received the conditionals part of the framework: at least 10 sessions of mental practice (step 2) and have practiced outside of supervised therapy time (Braun SM, van Haastregt JC, Beurskens AJ, Gielen X, Wade DT, Schols JM, submitted data, 2009).

Patients are not specifically taught to use a third or first person's view (11). During therapy imagery attempts and overt movements are combined: movements are performed to generate sensory information, which are then embedded in the imagery attempts to make them as vivid as possible. The proportions of actual movements and imagery attempts are based on individual preferences (12). Therapy with the therapist was recorded in pre structured patient files (content and time spend) and an imagery log was handed to the patient to register unguided mental practice (13).

\section{Control intervention}

To compensate for the unguided imagery training, patients in the control group were also encouraged to do 'homework', primarily practising tasks that they had difficulty with. They were asked to report unguided therapy in logs.

\section{Measures}

The measures used are shown in table one (next page). Selection was based on recommendations in the Dutch stroke guideline (9), methodological quality and being already part of the nursing home stroke assessments. 
Upon the patient's entry (T0) into the study the following patient characteristics were recorded: age, gender, time post stroke, cognitive level (Mini Mental State Examination, (14)), brain lesion site, self-judged ability to imagine motor acts, and the score on the Barthel Index (15).

The primary end-point was patient-perceived performance in activities practiced, because it seemed that mental practice would have most effect on movements that were actually mentally rehearsed (13). Performance of two 'standard' activities, drinking and walking and two individually chosen activities, one for the arm and one for the leg, were rated by the patient (and also by the therapist) on a score from 10 ('excellent') to 1 ('poor') (16). Six secondary study parameters/endpoints were used $(15,17-21)$.

Tab.1: Overview of used measures in this study.

\begin{tabular}{|c|c|c|}
\hline Data & Time & Subject of assessment \\
\hline \multicolumn{3}{|l|}{ Demographics } \\
\hline Age, gender, time post-stroke... & T0 & Comparison at baseline \\
\hline \multicolumn{3}{|l|}{ Primary outcome (16) } \\
\hline 10 point Likert scale (LS) & $\mathrm{T} 0, \mathrm{~T} 1, \mathrm{~T} 2$ & $\begin{array}{l}\text { Drinking and walking and two patient } \\
\text { specific tasks }\end{array}$ \\
\hline \multicolumn{3}{|l|}{ Secondary outcome } \\
\hline Motricity Index (MI) (19) & $\mathrm{T} 0, \mathrm{~T} 1, \mathrm{~T} 2$ & $\begin{array}{l}\text { Voluntary activity and strength in six limb } \\
\text { movements }\end{array}$ \\
\hline Barthel Index (BI) (15) & $\mathrm{T} 0, \mathrm{~T} 1, \mathrm{~T} 2$ & $\begin{array}{l}\text { Degree of independent performance of } \\
\text { daily activities }\end{array}$ \\
\hline Nine Hole Peg Test (NHPT) (21) & $\mathrm{T} 0, \mathrm{~T} 1, \mathrm{~T} 2$ & Speed of fine hand coordination \\
\hline Berg Balance Scale (BBS) (18) & $\mathrm{T} 0, \mathrm{~T} 1, \mathrm{~T} 2$ & Physical performance scale on balance \\
\hline Rivermead Mobility Index (RMI) (17) & T0, T1, T2 & $\begin{array}{l}\text { Staff-completed questionnaire on mobility } \\
\text { disability }\end{array}$ \\
\hline Ten metres walk time (TMW) (20) & $\mathrm{T} 0, \mathrm{~T} 1, \mathrm{~T} 2$ & Walking speed \\
\hline \multicolumn{3}{|l|}{ During six weeks intervention period } \\
\hline $\log$ & Daily & \\
\hline Interview & Between T1/T2 & Sample size (max. $\mathrm{n}=10)$ \\
\hline
\end{tabular}




\section{Statistical analysis}

The calculation of the sample size was based on the primary outcome measure, perceived performance of daily activities has been published elsewhere (7). Nineteen patients in each group were calculated, assuming a 50:50 random allocation (38 total). The goal of this study was to have 35 patients in each group to allow for drop-outs, loss to follow-up and uncertainty in the power calculation.

Group characteristics at baseline were presented using descriptive statistics. Differences at baseline were judged on clinical relevance (22). Adjustments in the analysis for possible imbalance at baseline were only made if the variable was established being prognostic for outcome: time post stroke, age and the Barthel Index (23).

The primary analysis was performed on an intention to treat basis of all patients as randomised and allocated to the experimental or control group. Raw data (means, SD) at the three measuring points were presented per group.

Generalized estimating equations (GEE), a longitudinal linear regression technique, to account for the dependency of the observations in time, was used to analyze effects of the intervention over time. Based on the goodness of fit test (the lowest number) we chose the GEE-correlation structure, which could be exchangeable, unstructured or autoregressive AR(1). Mean differences were calculated and the confidence interval $(95 \% \mathrm{CI})$ and the p-value given (24). The whole analysis was also repeated as a per protocol analysis.

\section{Results}

Two deviations from the original protocol took place (7). The EEG-measures were stopped after half a year because the additional time exceeded the estimated time considerably. The TUG was not performed to also reduce measuring time. One minor adjustment was made: a 10 point instead of 11 point Likert scale was used. A third location was included to increase recruitment.

In the three nursing homes together 391 patients with stroke in the sub acute phase of recovery were screened for participation, 65 were asked and 36 gave informed consent for the study, resulting in the random allocation of 18 in each group (fig. 1, next page). 


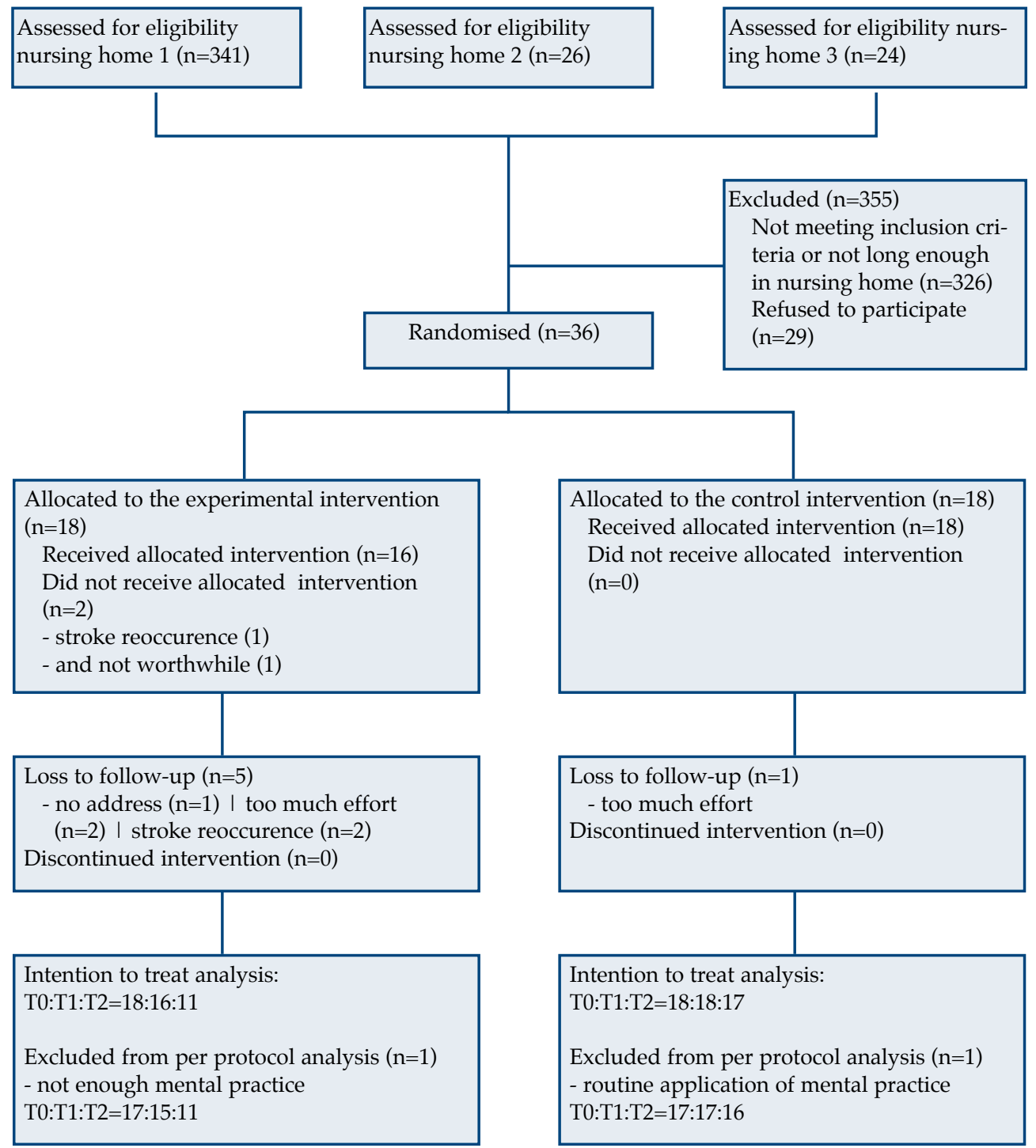

Fig. 1: Flow chart of participant recruitment, allocation and number of participants per intervention group and per measure point ( $\mathrm{T} 0=$ baseline, $\mathrm{T} 1=$ after intervention; six weeks, $\mathrm{T} 2=$ =follow-up; six months).

Two patients did not complete the entire six weeks of intervention (experimental group). Six patients were recorded as loss to follow-up (of which one from the control group).

\section{Comparability of treatment groups}

In table two (next page) the characteristics of the patients in both groups are described. The baseline differences on characteristics and outcome measures between groups were minimal or not considered to be clinically important. Therefore, no correction took place. 
Tab. 2: Baseline characteristics of the control and mental practice group.

\begin{tabular}{|c|c|c|}
\hline Characteristics & $\begin{array}{l}\text { Control group } \\
\mathrm{n}=18\end{array}$ & $\begin{array}{l}\text { Experimental group } \\
\mathrm{n}=18\end{array}$ \\
\hline Age, mean (SD) & $77,9(7,4)$ & $77,7(7,2)$ \\
\hline Time post stroke (weeks), mean (SD) & $4,8(3,3)$ & $6,1(2,7)$ \\
\hline Barthel Index & $12,2(5,4)$ & $11,2(4,1)$ \\
\hline Gender, number (\%) male/female & $9 / 9(50 \% / 50 \%)$ & $5 / 13(27,8 \% / 72,2 \%)$ \\
\hline$M M S E$, mean (SD) & $24,8(4,6)$ & $25,4(3,6)$ \\
\hline \multicolumn{3}{|l|}{ Affected brain side, number (\%) } \\
\hline Left & $9(50 \%)$ & $6(33,3 \%)$ \\
\hline Right & $8(44,4 \%)$ & $9(50 \%)$ \\
\hline Central/stam & $1(5,6 \%)$ & $3(26,7 \%)$ \\
\hline \multicolumn{3}{|l|}{ Ability to perform imagery $\quad(n=17)$} \\
\hline Not able to imagine & $1(5,9 \%)$ & $2(11,8 \%)$ \\
\hline A bit able to imagine (age images) & $6(35,3 \%)$ & $7(41,2 \%)$ \\
\hline Average ability to imagine & $3(17,6 \%)$ & $5(29,4 \%)$ \\
\hline Very well able to imagine (vivid images) & $7(41,2 \%)$ & $3(17,6 \%)$ \\
\hline
\end{tabular}

MMSE: Mini Mental State Examination | SD = Standard deviation

Ability to perform motor imagery: as judge by the patient him/herself

\section{Treatment, compliance, contamination and blinding}

Treatment with mental practice in the majority of cases could not be embedded in regular therapy time. Between 10 to 20 minutes were added by seven out of the 10 therapists to teach and monitor mental practice (Braun SM, van Haastregt JC, Beurskens AJ, Gielen X, Wade DT, Schols JM, submitted data, 2009).

Contamination on a therapist level did not occur; some therapists, however, were sceptical about mental practice. (Braun SM, van Haastregt JC, Beurskens AJ, Gielen X, Wade DT, Schols JM, submitted data, 2009).

Patients generally complied to instructions. Some patients however found it hard to generate kinesthetic feeling and a few couldn't at all (Braun SM, Beurskens AJ, Dalemans R, Schols JM, Wade, submitted data, 2010).

Reporting unguided mental practice in the logs turned out problematic. When patients used the $\operatorname{logs}(\mathrm{n}=12)$, therapists were not sure whether they had reported physical or mental practice. Four patients did not report unguided mental practice, which may be caused by the inability to write, 
forgetting or in fact not practising (Braun SM, van Haastregt JC, Beurskens AJ, Gielen X, Wade DT, Schols JM, submitted data, 2009).

On a patient level, no contamination took place. This was revealed by the questionnaires handed to the patients after the six week intervention period and at six months follow-up. (Braun SM, van Haastregt JC, Beurskens AJ, Gielen X, Wade DT, Schols JM, submitted data, 2009).

The check of the independent rater (MK) revealed that blinding on the measuring level was successful: correct guess of allocation was not above guessing chance.

\section{Effects of the interventions}

The self chosen activities by the patients for the arm and leg in the control and the experimental group are shown in table three.

Tab. 3: Self chosen activity for the lower and upper extremity by the patients.

\begin{tabular}{|c|c|c|c|c|}
\hline & Control group & & Experimental group & \\
\hline \multirow[t]{5}{*}{ Activity arm } & None & $\mathrm{n}=3(16,7 \%)$ & None & $\mathrm{n}=3(16,7 \%)$ \\
\hline & Move arm general & $\mathrm{n}=6(33,3 \%)$ & Move arm general & $\mathrm{n}=9(50 \%)$ \\
\hline & Writing & $\mathrm{n}=3(16,7 \%)$ & Writing & $\mathrm{n}=2(11,1 \%)$ \\
\hline & Special & $\mathrm{n}=0(0 \%)$ & Special & $\mathrm{n}=1(5,6 \%)$ \\
\hline & ADL/house hold & $\mathrm{n}=6(33,3 \%)$ & ADL/house hold & $\mathrm{n}=3(16,7 \%)$ \\
\hline \multirow[t]{6}{*}{ Activity leg } & None & $\mathrm{n}=1(5,6 \%)$ & None & $\mathrm{n}=0(0 \%)$ \\
\hline & Standing up/sitting & $\mathrm{n}=8(44,4 \%)$ & Standing up/sitting & $\mathrm{n}=9(50 \%)$ \\
\hline & Stairs & $\mathrm{n}=3(16,7 \%)$ & Stairs & $\mathrm{n}=1(5,6 \%)$ \\
\hline & Standing/balancing & $\mathrm{n}=4(22,2 \%)$ & Standing/balancing & $\mathrm{n}=4(22,2 \%)$ \\
\hline & Arm activity & $\mathrm{n}=1(5,6 \%)$ & Arm activity & $\mathrm{n}=0(0 \%)$ \\
\hline & Others & $\mathrm{n}=1(5,6 \%)$ & Others & $\mathrm{n}=4(22,2 \%)$ \\
\hline
\end{tabular}

All participant improved significantly on all outcome measures, except for the nine hole peg test at six weeks post intervention and were better still at follow-up than at baseline. However, in the control group four measures and in the experimental group two measures revealed a (slight) set back at T2 (follow-up) compared to T1 (post intervention).

Table four $(\mathrm{p} 133,134)$ shows the results from the intention to treat analyses for the primary and secondary outcome measures. No significant differences were found between the two groups on any outcome measure at any point. There were also no differences in effects between the outcomes on the Likert scale scored by the therapists (data not shown here). 


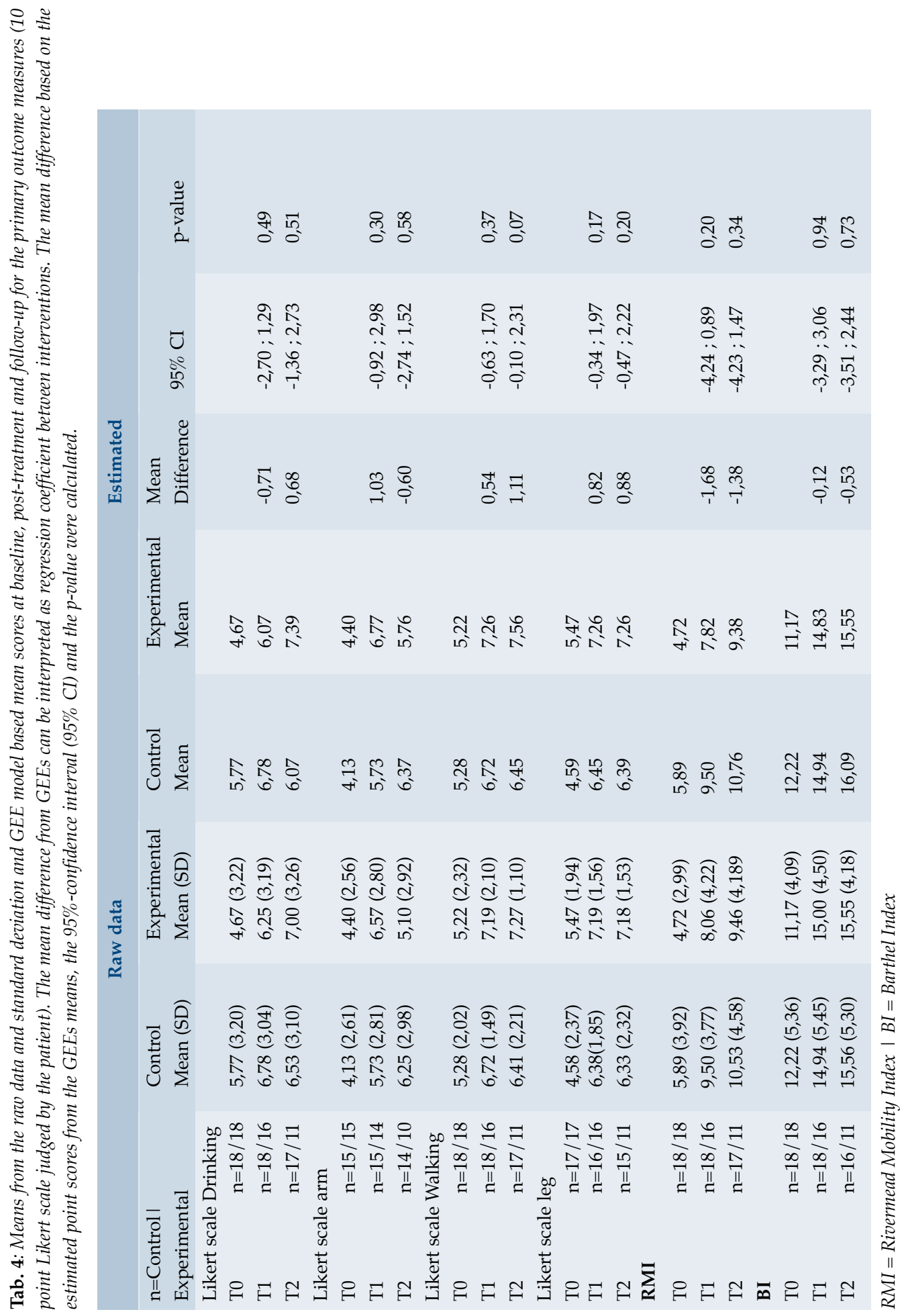




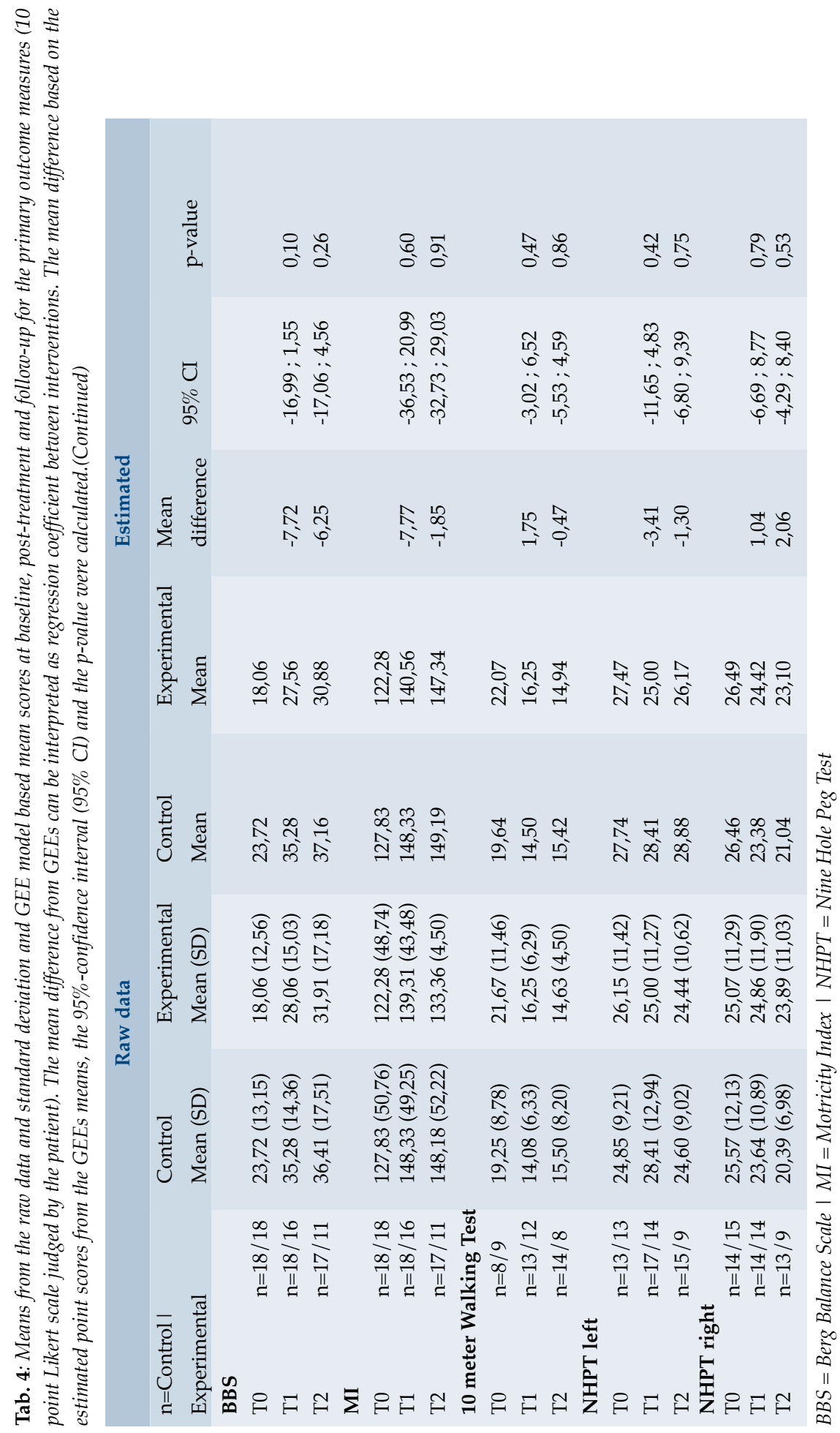


All but one participant in each group received the intervention according to protocol (Braun SM, van Haastregt JC, Beurskens AJ, Gielen X, Wade DT, Schols JM, submitted data, 2009) and both were excluded from the per protocol analyses. The results from the per protocol analyses (data not shown) were similar to the results of the intention to treat analyses.

\section{Discussion}

We investigated the potential effect of mental practice embedded in routine multi professional care in nursing homes on the performance of daily activities in patients after stroke in the sub acute phase of recovery. In this study, no significant differences were found between the control and experimental group on any of the outcome measures at any measuring point. Not even a trend was visible in favour of the experimental intervention.

We prepared this trial carefully with many preliminary studies. Based on the literature and evidence (4) we developed an intervention framework (10) that should do justice to routine care. The Structural Dimension Analysis of Motor memory (SDA-M) (25) and the outcome measures were chosen specifically for this intervention and population (7). All steps were pretested in a feasibility study and applied in a case series (26). Although the pilot study revealed promising results, we were not able to repeat the effect of mental practice in stroke patients in this trial. Several reasons might account for this outcome: the chosen population, intervention and outcome measures.

In previous RCTs, mental practice applied in stroke patients, both in the chronic (27-29) and sub acute phase of recovery (30), generally had a significant better outcome than the control intervention. Our study resembles the study by Liu and co-workers the most: they also gave a complex mental practice intervention to stroke patients in the sub acute phase of recovery but, contrary to our trial, with success (30).

Our intervention differed from others published ones with regard to the intervention period (relatively long) and we incorporated an instruction and training period for the therapists and patients (4). Other studies had either patients in the chronic phase of recovery or a mixed population and all other interventions were less complex but again effective. Only Dijkerman and co-workers reported no effects of a home based mental practice intervention on placing buttons on a line in stroke patients in the chronic phase of recovery (31). 
The population and setting

We chose a nursing home setting because a majority of older stroke patients in the Netherlands receive their rehabilitation in a nursing home (32) and so it was clinically important to study this group.

Our experience demonstrates the difficulty of undertaking research in the nursing home setting in the Netherlands: we recruited under $10 \%$ of all admitted patients during 1,5 years, contrary to the expected $40 \%$ (7). We probably missed the patients most likely to benefit because patients going home within a few weeks and patients being transferred to a specialized rehabilitation centre were not included in the trial. This meant that the patients recruited for this trial were a specific and frail sub group.

The sub acute population was specifically chosen for two main reasons. Plasticity of the brain in patients in the sub acute phase of recovery is assumed to be bigger $(33,34)$ and therefore creating correct mental representations of movements through movement imagery would have a bigger chance compared to the later phases of recovery. Second, the patient would be perhaps more able to remember the correct feeling and performance of skills from before the stroke and therefore generate more kinesthetic and visual information during mental practice.

\section{The intervention}

Introducing a complex intervention, embedded in multidisciplinary care in a population of persons with complex pathology poses challenges (35). Therapists reported difficulties in coping with the decisions needed within the framework and in not knowing for sure whether patients were practising mentally or not (especially outside of therapy). The framework may not have been sufficiently dogmatic for the therapists (or patients). If variation in the received intervention content would have lead to a distorted picture, the per protocol analyses should have revealed it (but did not). It was more difficult to change routine care in some therapists, then we thought.

It could also be argued that allowing clinical judgement to determine aspects of therapy leads to a non-standard approach. However we would argue that a non-standard approach is essential because patients are not standard either in their losses or in their abilities: a rigid dogmatic approach would be unrealistic.

Furthermore, even though the experimental group probably received more therapy input and 12 patients reported unguided mental practice up to an additional 30 hours, it is still uncertain that the group received sufficient additional input (Braun SM, Beurskens AJ, Dalemans R, Schols JM, Wade, submitted data, 2010). 
Power, and the outcome measures

Patients in both groups significantly improved on almost all outcome measures at almost all measuring points, but the differences in improvements between the two groups were small: sometimes the control and sometimes the experimental group improved more. The improvement is due to natural recovery combined with a bundle of care. Possibly the additional effects of mental practice, if any, are not measurable within the general recovery change seen. Our power calculation was based on overall change, and realistically the additional therapy may only have a small additional effect. Our study was probably underpowered to detect any additional effect. Almost all published trials have reported significant differences. Generally it is easier to get positive results published, especially if the studies have relatively small sample size (publication bias).

Another reason why we were unable to measure effects may be that mental practice has benefits not captured by our physical measures (36). Seven stroke patients from this trial were interviewed individually with regard to their mental practice habits (Braun SM, Beurskens AJ, Dalemans R, Schols JM, Wade, submitted data, 2010). They reported increased feeling of autonomy. Similar 'soft' effects of mental practice have been described in sports (37) and some information on stroke is available as well (38).

It is worth noting that negative side effects of imagery have recently been reported in patients with chronic pain (39). We did not have any reported, but we did not specifically ask.

\section{Practical and research implications}

We do not recommend to implement mental practice in the manner we described (10) in this specific sub population of stroke patients with the intention to enhance or accelerate recovery more. At present it should only be used in the context of research.

Aspects that could be researched are:

\section{Can patients be selected?}

When we planned the trial two and a half years ago, little was known about which patients were most likely to benefit from mental practice $(4,5)$. Meanwhile two interesting ways to assess a patient's imagery ability have been published: the hand rotation test (40) and the Kinesthetic and Visual Imagery Questionnaire (41). These tests might help in selecting the patients more appropriate for mental practice.

\section{How should patients be taught mental practice?}

An interesting point brought up by Milton and co-workers (11), based on the work by Masters et al (42) and Beilock et al (43), is that paying attention to a skill that has been automated, might lead to performance breakdown. Reinvesting in movements that are already in a late stage of 
motor learning could be counter-productive: this is another risk we did not measure. Imagery content might therefore need to be adjusted to the complexity of the skill and might be better not used in the later stages of motor learning.

In this study, we did not explicitly tell our patients to image in the first or third person's view nor did we encourage either visual imagery or kinesthetic imagery. There is evidence that both ways of imagery are effective in clinical practice (11). Generally, kinesthetic imagery is seen as the most potential form but there seems to be a shift from the first person's view towards the third person's view when one grows older (44). Because of sensory loss and the high age of most stroke patients, the third persons view or visualization of the movement will probably be more common.

\section{Conclusion}

We conclude that the evidence concerning the use of mental practice in stroke rehabilitation is still only based on a small number of trials with relatively small numbers of patients. Our study does not support routine use of mental practice, and emphasizes the need for both more developmental and evaluative research.

\section{Acknowledgments}

We thank all involved therapists and patients for participating in the trial. We appreciate the help of Machteld Schravendeel with the data collection and Marieke Spreeuwenberg, MSc, Zuyd University of applied sciences, with the statistical analysis. The study was funded by NutsOhra (SNO T-0702-70), VGZ (ZIP/HH/ME/07.190) and the Mobiliteitsfonds (PR0601-B-E).

\section{References}

1. Kwakkel G, van Peppen R, Wagenaar RC, Wood Dauphinee S, Richards C, Ashburn A, et al. Effects of augmented exercise therapy time after stroke: a meta-analysis. Stroke 2004;35: 2529-39.

2. Jackson PL, Lafleur MF, Malouin F, Richards C, Doyon J. Potential role of mental practice using motor imagery in neurologic rehabilitation. Arch Phys Med Rehabil 2001;82:1133-41.

3. Bernhardt J, Chan J, Nicola I, Collier JM. Little therapy, little physical activity: rehabilitation within the first 14 days of organized stroke unit care. J Rehabil Med 2007;39:43-8.

4. Braun SM, Beurskens AJ, Borm PJ, Schack T, Wade DT. The effects of mental practice in stroke rehabilitation: a systematic review. Arch Phys Med Rehabil 2006;87:842-52. 
5. Sharma N, Pomeroy VM, Baron JC. Motor imagery: a backdoor to the motor system after stroke? Stroke 2006;37:1941-52.

6. Langhorne P, Coupar F, Pollock A. Motor recovery after stroke: a systematic review. Lancet Neurol 2009;8:741-54.

7. Braun SM, Beurskens AJ, van Kroonenburgh SM, Demarteau J, Schols JM, Wade DT. Effects of mental practice embedded in daily therapy compared to therapy as usual in adult stroke patients in Dutch nursing homes: design of a randomised controlled trial. BMC Neurol 2007;7:34.

8. Altman DG, Schulz KF, Moher D, Egger M, Davidoff F, Elbourne D, et al. The revised CONSORT statement for reporting randomized trials: explanation and elaboration. Ann Intern Med 2001;134:663-94.

9. Commissie CVA-revalidatie. Revalidatie na een beroerte, richtlijnen en aanbevelingen voor zorgverleners. Den Haag: Nederlandse Hartstichting; 2001

10. Braun SM, Kleynen M, Schols JM, Schack T, Beurskens AJ, Wade DT. Using mental practice in stroke rehabilitation; a framework. Clin Rehabil 2008;22:579-591.

11. Milton J, Small SL, Solodkin A. Imaging motor imagery: methodological issues related to expertise. Methods 2008;45:336-41.

12. Malouin F, Richards CL, Doyon J, Desrosiers J, Belleville S. Training mobility tasks after stroke with combined mental and physical practice: a feasibility study. Neurorehabil Neural Repair 2004;18:66-75.

13. Gould D, Damarjian N, Greenleaf C. Imagery training for peak performance. In: Raalte van J, Brewer WB, editors. Exploring sport and exercise psychology. Washington (DC): Am Psychol Assoc; 2002. p 49-74.

14. Tombaugh TN, McIntyre NJ. The mini-mental state examination: a comprehensive review. J Am Geriatr Soc 1992;40:922-35.

15. Kalra L, Eade J. Role of stroke rehabilitation units in managing severe disability after stroke. Stroke 1995;26:2031-4.

16. Wewers ME, Lowe NK. A critical review of visual analogue scales in the measurement of clinical phenomena. Res Nurs Health 1990;13:227-36.

17. Lennon S, Johnson L. The modified rivermead mobility index: validity and reliability. Disabil Rehabil 2000;22:833-9.

18. Blum L, Korner-Bitensky N. Usefulness of the Berg Balance Scale in stroke rehabilitation: a systematic review. Phys Ther 2008;88:559-66.

19. Garraway WM, Akhtar AJ, Prescott RJ, Hockey L. Management of acute stroke in the elderly: preliminary results of a controlled trial. Br Med J 1980;280:1040-3. 
20. Hankey GJ, Deleo D, Stewart-Wynne EG. Stroke units: an Australian perspective. Aust N Z J Med 1997;27:437-8.

21. Oxford Grice K, Vogel KA, Le V, Mitchell A, Muniz S, Vollmer MA. Adult norms for a commercially available Nine Hole Peg Test for finger dexterity. Am J Occup Ther 2003;57: 570-3.

22. Assmann SF, Pocock SJ, Enos LE, Kasten LE. Subgroup analysis and other (mis)uses of baseline data in clinical trials. Lancet 2000;355:1064-9.

23. Kollen B, Kwakkel G, Lindeman E. Functional recovery after stroke: a review of current developments in stroke rehabilitation research. Rev Recent Clin Trials 2006;1:75-80.

24. Twisk JW. Applied longitudinal data analysis for epidemiology. Cambridge: Cambridge university press; 2007.

25. Page SJ. Imagery improves upper extremity motor functions in chronic stroke patients with hemiplegia: a pilot study. Occup Ther J Res 2000;20:200-15.

26. Page SJ, Levine P, Leonard AC. Effects of mental practice on affected limb use and function in chronic stroke. Arch Phys Med Rehabil 2005;86:399-402.

27. Page SJ, Levine P, Sisto S, Johnston MV. A randomized efficacy and feasibility study of imagery in acute stroke. Clin Rehabil 2001;15:233-40.

28. Liu KP, Chan CC, Lee TM, Hui-Chan CW. Mental imagery for promoting relearning for people after stroke: a randomized controlled trial. Arch Phys Med Rehabil 2004;85:1403-8.

29. Dijkerman HC, Letswaart M, Johnston M, MacWalter RS. Does motor imagery training improve hand function in chronic stroke patients? A pilot study. Clin Rehabil 2004;18:538-49.

30. Braun SM, Schack T, Marcellis RG, Oti KC, Schols JM, Wade DT, Beurskens AJ. Representations of motor actions in stroke patients and healthy elderly. Clin Rehabil 2007;21:822-32.

31. Kleynen M, Schoenmakers M, Braun SM. Stel je voor, het werkt. Mentale training bij een chronische CVA patiënt: een case report. Ned Tijdschr Fysiother 2009;119:48-54.

32. Schols JM, Crebolder HF, van Weel C. Nursing home and nursing home physician: the Dutch experience. J Am Med Dir Assoc 2004;5:207-12.

33. Burke SN, Barnes CA. Neural plasticity in the ageing brain. Nat Rev Neurosci 2006;7:30-40.

34. Kleim JA, Jones TA. Principles of experience-dependent neural plasticity: implications for rehabilitation after brain damage. J Speech Lang Hear Res 2008;51:225-39.

35. Craig P, Dieppe P, Macintyre S, Michie S, Nazareth I, Petticrew M. Developing and evaluating complex interventions: the new Medical Research Council guidance. Bmj 2008;337:a1655.

36. Johnson-Frey SH. Stimulation through simulation? Motor imagery and functional reorganization in hemiplegic stroke patients. Brain $\operatorname{Cog} n$ 2004;55:328-31. 
37. Martin K, Moritz S, Hall C. Imagery use in sports: a literature review and applied model. The Sports Psychologist 1999;13:245-68.

38. Simmons L, Sharma N, Baron JC, Pomeroy VM. Motor imagery to enhance recovery after subcortical stroke: who might benefit, daily dose, and potential effects. Neurorehabil Neural Repair 2008;22:458-67.

39. Moseley GL. Graded motor imagery is effective for long-standing complex regional pain syndrome: a randomised controlled trial. Pain 2004;108:192-8.

40. Parsons LM, Gabrieli JD, Phelps EA, Gazzaniga MS. Cerebrally lateralized mental representations of hand shape and movement. J Neurosci 1998;18:6539-48.

41. Malouin F, Richards CL, Jackson PL, Lafleur MF, Durand A, Doyon J. The Kinesthetic and Visual Imagery Questionnaire (KVIQ) for assessing motor imagery in persons with physical disabilities: a reliability and construct validity study. J Neurol Phys Ther 2007;31:20-9.

42. Masters RSW, Maxell JP. The theory of reinvestment. International Review of Sports and Exercise Psychology 2008;1:160-83.

43. Beilock SL, Carr TH, MacMahon C, Starkes JL. When paying attention becomes counterproductive: impact of divided versus skill-focused attention on novice and experienced performance of sensorimotor skills. J Exp Psychol Appl 2002;8:6-16.

44. Mulder T, Hochstenbach JB, van Heuvelen MJ, den Otter AR. Motor imagery: the relation between age and imagery capacity. Hum Mov Sci 2007;26:203-11. 


\section{Chapter eight}

Effects of mental practice in patients with Parkinson's disease: results from a multi-centre randomised controlled trial

Braun SM, Beurskens AJ, Kleynen M, Schols JM, Wade DT

(Submitted 2010) 


\section{Abstract}

Background: Mental practice as a novel and additional therapy for people with Parkinson's disease in the community might be effective.

Objective: The goal of this study was to investigate its effectiveness at improving mobility and to assess whether patients in less severe stages of the disease are more eligible and benefit more.

Methods: A multi-centre randomised controlled trial with a six week intervention period was conducted. Both groups received therapy as usual with additionally either relaxation (control group) or mental practice (experimental group). Outcome was assessed at six weeks and three months with: the patient- and therapist perceived effect on walking performance (VAS), Timed Up and Go, 10m Walking Test and time needed for Stance-Lying-Stance. Primary analysis was performed using intention to treat and was repeated as a per protocol and sub group analysis (Hoehn and Yahr < stage 3). Generalized estimating equations were used to analyze effects.

Results: Forty-seven participants (average age 69,4 years, SD 7,6) in different disease stages were assigned to the control $(n=22)$ and experimental group $(n=25)$. No effect in favour of the mental practice intervention on any outcome measure could be detected at any of the measuring points. In the sub group analysis the experimental group improved more than the control group (not significant).

Conclusions: At this point there is insufficient evidence to support the routine use of mental practice as part of the rehabilitation package for people with Parkinson's disease. Further research should focus on larger research populations with less severe patients. 


\section{Effects of mental practice in patients with Parkinson's disease: results from a multi-centre randomised controlled trial}

\section{Background}

Patients with Parkinson's disease are usually treated with dopaminergic medication. To cope with motor control problems, many patients are additionally treated by a physiotherapist, even in early stages of the disease (1). The therapy is targeted at improving, maintaining or delaying problems with gait, transfers, posture, balance and general physical condition (1). Cognitive deficits (e.g. problems concentrating, attention problems) are also common in patients with Parkinson's disease $(2,3)$. There is evidence that therapy including cueing strategies helps to improve, maintain or delay motor control problems $(1,4-6)$. It has been hypothesized that movement imagery might have an added value in patients with Parkinson's disease because it targets the conscious control of movement through cognitive strategies, which is generally recommended in National guidelines (7).

Athletes have used all sort of cognitive skills to improve motor performance. The use of mental practice in athletes has been subject to research for several decades (8). Recently mental practice has been used in different patient populations as an additional therapy (9) on the understanding that it increases practice of skilled movements. The following descriptions are used (10): 'During mental practice an internal representation of the movement is activated and the execution of the movement repeatedly mentally simulated within a chosen context. This mental simulation takes place in absence of bodily activity. It is used for the goal-oriented improvement or stabilization of a given movement'. Movement imagery is the underlying technique for mental practice and is described as 'imagining oneself undertaking the skilled movement without actually doing the movement'.

The potential of mental practice in neurological rehabilitation has been described by Jackson and co-workers (11). However, when techniques from other research areas are used, there is a need for a theoretical basis on which possible effects may be explained (12). Brain imaging research in healthy subjects has shown that during vivid imagery of a specific movement almost the same brain areas are active as during overt movement (13). Fundamental research in patients has mainly been done with patients suffering from stroke (14-18) and this kind of research with patients with Parkinson's disease shows that some but not all are able to perform imagery (1921). 
Piloting and feasibility studies with mental practice have taken place in different patient populations. There is some evidence that mental practice might help patients with conditions such as chronic pain, cancer and orthopaedic pathologies $(9,10,14)$.

However, the majority of clinical research has been performed in stroke patients (9). Initially the focus of mental practice was on the improvement of arm-hand-functions $(10,14)$, but recently more studies have taken place assessing the possible effects of mental practice on locomotor tasks (22). There is some evidence that several different mental practice interventions might work (10). It seems important however, to tailor the mental practice content to the abilities of the patient, as neurological conditions can influence the ability of patients to generate vivid images (cognitive level), decrease kinesthetic input and limit physical performance (23).

Only few clinical studies have been conducted in patients with Parkinson's disease $(24,25)$. Results show some controversy on what effects a mental practice intervention might have. Recently however, promising results of a randomized clinical trial of reasonable size and duration have been published by Tamir and co-workers (25). In this randomised controlled trial (RCT) mental practice was used to assess whether it would have a larger effect on mobility tasks.

Mental practice in patients with Parkinson's disease might have a positive effect, but more research needs to be done to be sure. We therefore performed a randomized controlled study in patients with Parkinson's disease in order to assess the effects of a mental practice framework, which can be tailored to the patients' abilities.

The overall aim of this research project was to investigate the therapeutic effect of mental practice embedded in usual physiotherapy on the improvement of locomotor tasks of patients with Parkinson's disease.

As we included patients with a wide range in severity of the disease, the second research question was to assess whether patients in less severe stages of Parkinson's disease (below stage 3 on the Hoehn and Yahr scale) are more eligible to perform mental practice and therefore might benefit more from it (sub group analysis).

\section{Methods}

This study is registered at www.trialregister.nl (NTR1735). The study protocol was approved by the local medical ethical committee of the Atrium medical centre, Orbis medical centre and HsZuyd and was conducted over a one year period. Patients included in the trial gave informed 
consent and were treated at one of five locations: three community practices, rehabilitation day treatment in a nursing home or hospital (all were out patients).

Decentralized randomisation with envelopes took place by an independent third party blind for the patient's characteristics, based on a (size four block) randomisation schedule (26). No stratification took place. The randomisation procedure was the same for all five locations and took place on an individual level. After baseline measurements, the therapists were notified to which group the patient was assigned.

The patients were not blinded to the treatment they received as they were aware of the treatment content. And therapists knew, as they taught the patient.

\section{Study population}

Patients with Parkinson's disease entering the trial had to meet following inclusion criteria:

a) clinically diagnosed adult patients with Parkinson's disease;

b) sufficient cognitive level and communication skills to engage in mental practice. Taken into account were: the clinical judgment of the treating therapist, support from family and the score on the mini mental state examination (MMSE (27)).

Patients who had conditions such as rheumatic diseases or dementia prior to Parkinson's disease onset sufficient to cause persistent premorbid disability were excluded.

\section{Interventions}

The patients recruited were already receiving physiotherapy according to the Dutch guidelines for patients with Parkinson's disease (7), some on a one-to-one basis and some in groups. This pre-existing treatment was continued. The allocated 'new' treatment, based on the randomisation schedule (individual level), was incorporated into the patient's program. All patients received six weeks of physiotherapy, leaving their own therapy frequency and organization unchanged. Patients received either one hour of physiotherapy per week (groups) or two sessions of half an hour per week (individuals). In both cases this lead to an amount of six hours with no increase in contact time with the therapist. If patients were treated on an individual basis for half an hour, ten minutes were spend on mental practice or relaxation, in group sessions of one hour, the time was increased to 20 minutes. To control for attention, patients in both groups were encouraged to use their allocated new therapy technique outside their normal treatment. Therapy with the therapist was recorded in pre-structured patient files (content and time spent) and a log was handed to register unguided practice.

Supervision by the researcher during the intervention period took place through checking of the patient files. 


\section{Experimental intervention - mental practice of a locomotor task}

Therapists were trained to teach and monitor mental practice according to the framework in which four steps are distinguished (23): (1) explaining the concept, (2) developing imagery techniques, (3) applying mental practice and (4) and consolidating. Unlike a fixed treatment regime, the framework allowed the physiotherapist to tailor the imagery content to the patients' abilities and preferences. Examples of tailoring are the chosen view and the ratio of overt and imagery attempts of movements. Patients were not taught to use a specific view (first person's view: as if looking through their own eyes or third person's view: as if looking at oneself from a distance) (13). During therapy imagery attempts and overt movements were combined: movements were performed to generate sensory information. This information was then embedded in the imagery attempts to make them as vivid as possible. The proportions of actual movements and imagery attempts were based on individual preferences (28). Details on the intervention have been published elsewhere (23). The main goal of the mental practice intervention was to improve the locomotor tasks like walking, standing up from a chair, or the floor.

\section{Control intervention - progressive muscle relaxation}

The control therapy was used to control for attention. The control group received therapy according to the Dutch guidelines (7) with relaxation therapy being incorporated into each session (same amount as mental practice in the experimental group). Relaxation was chosen to enable comparison with the RCT by Tamir and co-workers (25) and followed the principles of progressive muscle relaxation (Jacobson (29)). Patients were encouraged to do relaxation homework outside of therapy as well, which consisted of unguided progressive muscle relaxation or listening to a relaxation $\mathrm{CD}$.

\section{Measures}

Table one (next page) gives an overview of the collected data and used measures. The following patient characteristics were recorded: age, gender, time post diagnosis Parkinson's disease, cognitive level (MMSE (27)), Hoehn and Yahr stage (3) and the use of walking aids.

Mental practice should have the most effects on the movement that is actually mentally rehearsed $(8,30-32)$. Improvement of these activities should therefore be assessed, for which a visual analogue scale (VAS (33)) was used. We asked each patient to complete a VAS on the locomotor task they were practising, for instance their walking performance, where the anchor points were ' 0 ' (poor performance) to ' 100 ' (perfect, effortless and smooth performance of walking). 
Data from two measures from the Dutch guidelines, used routinely by the therapists were used: timed up and go (TUG) and 10m walking test (10m WT) (34-38).

The TUG measures the time a patient needs to stand up from a chair, walk three meters at a comfortable speed, turn around, walk back and sit down. The patient is allowed to use his/her own walking aids, but no physical assistance may be given by the researcher or therapist. The test is practical and simple. The internal consistency, reliability, validity and responsiveness are sufficient $(35,37,38)$.

The $10 \mathrm{~m}$ WT can be used in patients able to walk independently with or without walking aids and/or orthoses. Patients should walk at a comfortable speed. The test is reliable, valid and responsive (34). Furthermore, a significant relation between the comfortable walking speed during the test and the quality with which patients walk has been established (36).

One other measure was included to enable comparison with the trial by Tamir and co-workers (25): the time needed to lie down from a standing position and get up again (stance-lying-stance, SLS). No psychometric data of this measure are known.

Tab.1: Overview of used measures in the Parkinson's study.

\begin{tabular}{|c|c|c|}
\hline Data & Time & Subject of assessment \\
\hline $\begin{array}{l}\text { Demographics } \\
\text { Age, gender, time post-PD, } \\
\text { MMSE (27), Hoehn and Yahr } \\
\text { stage (1-5) }\end{array}$ & T0 & Comparison at baseline \\
\hline $\begin{array}{l}\text { Outcome measures } \\
\text { VAS: walking ( } 33 \text { ) }\end{array}$ & $\mathrm{T} 0, \mathrm{~T} 1, \mathrm{~T} 2$ & $\begin{array}{l}0-100 \mathrm{~mm} \text { scale: ' } 0 \text { ' not able to perform the activity to } \\
' 100^{\prime} \text { perfect effortless performance of walking }\end{array}$ \\
\hline Timed Up and Go $(35,37,38)$ & $\mathrm{T} 0, \mathrm{~T} 1, \mathrm{~T} 2$ & $\begin{array}{l}\text { Time needed to stand up, walk } 3 \text { meters, turn, walk } \\
\text { back and sit down, was measured }\end{array}$ \\
\hline 10m Walking Test $(34,36)$ & $\mathrm{T} 0, \mathrm{~T} 1, \mathrm{~T} 2$ & Time needed to walk 10 meters was measured \\
\hline Stance-Lying-Stance & $\mathrm{T} 0, \mathrm{~T} 1, \mathrm{~T} 2$ & $\begin{array}{l}\text { Time needed to stand up from a lying position, and lie } \\
\text { down again was measured. }\end{array}$ \\
\hline $\begin{array}{l}\text { During } 6 \text { weeks intervention } \\
\text { period } \\
\text { Log }\end{array}$ & $\begin{array}{l}\text { Daily during } \\
6 \text { weeks }\end{array}$ & A new log was handed every week. \\
\hline $\begin{array}{l}\text { Afterwards (between } T 1 \text { and } T 2 \text { ) } \\
\text { Interview }\end{array}$ & Once & $\begin{array}{l}\text { All therapists and } 12 \text { patients were interviewed and } \\
\text { all were handed a questionnaire to assess the } \\
\text { feasibility of the mental practice program. }\end{array}$ \\
\hline
\end{tabular}

$T 0=$ at baseline $\mid T 1=$ after six week intervention period $\mid T 2=$ at follow up at 3 months MMSE = Mini Mental State Examination | VAS =visual analogue scale 
The data on outcome measures were collected by an independent, blinded assessor. Data were collected in the two weeks before starting (T0; baseline), during weeks 7-8 (T1; after the six week intervention period) and during weeks 12-13 (T2; after three months at follow up). In order to reduce the influence of fluctuating performance associated with Parkinson's disease, data were collected on three separate days during each of the three assessment periods (T1, T2, T3) and on each day each test was performed three times. For each patient we used the mean score on each measure for the measuring period: potentially this was the mean of nine values although some patients had less measures. The VAS measure was only measured once in each assessment period.

\section{Sample size calculation}

The calculation of the sample size was based on the VAS and on the expectation that patients were either in a steady state or would become worse during the intervention period. Thus we anticipated that any measured change would be attributable to the intervention. A difference of 20/100 on the $0-100$ visual analogue scale between the two groups seemed a reasonable change to aim for $(\mu 1=2.0)$. There is no literature available on the standard deviation of the VAS in a Parkinson's population, but in other pathologies it is between 1.5 and 3.0. In this sample size calculation we used a standard deviation of $2.25(\sigma=2.25)$. The power of this study was set at $90 \%$ $(\beta=0.10)$. The level of significance was set at $5 \%(\alpha=0.05)$. The sample size calculation formula used was: $\quad \mathrm{N} 1=\mathrm{N} 2=\left(\mathrm{z}_{1-\beta}+\mathrm{z}_{1-\alpha / 2}\right)^{2} *\left(\left(\sigma_{1}{ }^{2}+\sigma_{2}{ }^{2}\right) /\left(\mu_{1}-\mu_{2}\right)^{2}\right)$

Nineteen patients in each group were calculated, assuming a 50:50 random allocation. The goal of this study was to have 25 patients in each group to allow for drop-outs, loss to follow-up and uncertainty in the power calculation.

\section{Statistical analysis}

Group characteristics at baseline were presented in descriptive statistics: means and standard deviations (SD) for continuous variables and absolute numbers of participants and percentages for categorical variables. Differences between groups with regard to baseline characteristics were judged on clinical relevance (39) and calculated (t-test or Chi-square).

Generalized estimating equations (GEE), a longitudinal linear regression technique, to account for the dependency of the observations in time, were used to analyze the effects of the intervention (40) at T1 and T2. GEE was used because of the dependency of observations across time within patients and because the time frames between the baseline and $\mathrm{T} 1$ and between $\mathrm{T} 1$ and $\mathrm{T} 2$ were not equal. In addition, the use of GEE would ensure maximum power of the results. As level 1 variable we used the IDENTIFICATION CODE and as level 2 variable we used TIME. 
As outcome measures, we used percentage change scores, to correct for differences between groups at baseline on outcome measures. Percentage change scores from baseline were calculated at $\mathrm{T} 1$ and at follow up $((\mathrm{Tx}-\mathrm{T} 0) / \mathrm{T} 0 * 100 \%)$ for all outcome measures (VAS patient, VAS therapist, TUG, 10mWT, LSL). As independent variable we included TIME, INTERVENTION and the interaction TIME $x$ INTERVENTION. Adjustments in the analysis for possible confounding due to imbalance at baseline were made for variables known to be prognostic for outcome, time post diagnosis of Parkinson's disease, and age (1). (As no differences were found, the analysis was not corrected for confounding). Mean difference in difference of percentage change scores were estimated by the model and the confidence interval (95\% CI) and p-value given. Normal distribution of the data on the calculated change scores of the outcome measures was visually checked (Q- Q Plot). P-values $<0.05$ were considered significant. Three analyses with GEE took place. The primary analysis of the effect of intervention was performed on the entire research population on an intention-to-treat basis. The second analysis was a per protocol analysis: from the entire population, only patients who received $60 \%$ of the guided therapy and reached at least step 2 of the mental practice framework were included. As a third step, a sub-group analysis of the initial population (intention-to-treat) was performed on patients with a Hoehn and Yahr stage below three, who were hypothesized to be more eligible for mental practice (2). 


\section{Results}

Figure one gives an overview of participant recruitment, allocation and flow through the trial.

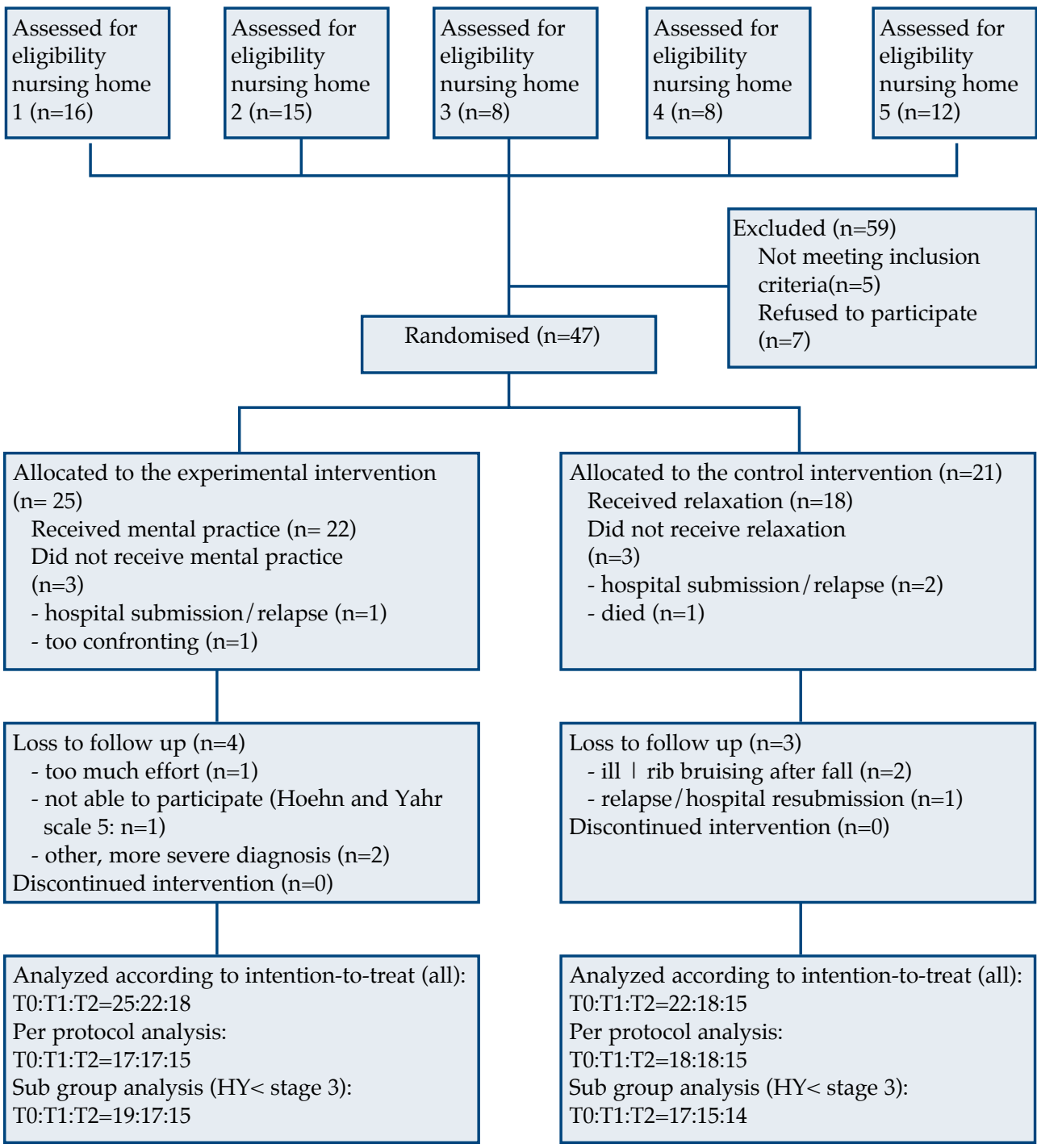

Fig. 1: Flow chart of participant recruitment, allocation and number of participants per intervention group and per measure point ( $\mathrm{T} 0=$ baseline, $\mathrm{T} 1=$ after intervention; six weeks, $\mathrm{T} 2=$ follow up; three months, $\mathrm{HY}=$ Hoehn and Yahr stage).

\section{Comparability of treatment groups}

In table two (next page) the characteristics of the patients in both groups are described, both for the entire group and sub group. In both cases, groups were comparable. 
Tab. 2: Patient characteristics of the entire study population per group.

\begin{tabular}{|c|c|c|c|c|}
\hline & \multicolumn{2}{|c|}{$\begin{array}{c}\text { All participants } \\
\text { (intention to treat analysis) }\end{array}$} & \multicolumn{2}{|c|}{$\begin{array}{c}\text { Participants with Hoehn and Yahr } \\
\text { < stage three } \\
\text { (sub group analysis) }\end{array}$} \\
\hline & $\begin{array}{l}\text { Control group } \\
\qquad(\mathrm{n}=22)\end{array}$ & $\begin{array}{l}\text { Experimental group } \\
\qquad(\mathrm{n}=25)\end{array}$ & $\begin{array}{l}\text { Control group } \\
\qquad(\mathrm{n}=17)\end{array}$ & $\begin{array}{l}\text { Experimental group } \\
\qquad(\mathrm{n}=19)\end{array}$ \\
\hline Age & 69,0 years $(\mathrm{SD} 7,6)$ & 69,8 years $(\mathrm{SD} 7,7)$ & 67,9 years $(\mathrm{SD} 7,5)$ & 69,4 years $(\mathrm{SD} 8,2)$ \\
\hline $\begin{array}{l}\text { Time post } \\
\text { diagnosis } \\
\text { PD }\end{array}$ & 6,6 years $(\mathrm{SD} 7,8)$ & 5,2 years (SD 5,0) & 5,0 years $(\mathrm{SD} 7,1)$ & 4,6 years (SD 4,1) \\
\hline MMSE & $27,0(\mathrm{SD} 2,0)$ & $27,4(\mathrm{SD} 2,9)$ & $26,9(\mathrm{SD} 2,0)$ & $27,9(\mathrm{SD} 2,8)$ \\
\hline $\begin{array}{l}\text { Male: } \\
\text { Female } \\
\text { Hoehn/ }\end{array}$ & $15: 7$ & $17: 8$ & $11: 6$ & $12: 7$ \\
\hline Yahr & $\mathrm{n}=3(14 \%)$ & $\mathrm{n}=6(24 \%)$ & $\mathrm{n}=3(18 \%)$ & $\mathrm{n}=6(32 \%)$ \\
\hline 1 & $\mathrm{n}=5(23 \%)$ & $\mathrm{n}=1(4 \%)$ & $\mathrm{n}=5(29 \%)$ & $\mathrm{n}=1(5 \%)$ \\
\hline $1-2$ & $\mathrm{n}=6(27 \%)$ & $\mathrm{n}=8(32 \%)$ & $\mathrm{n}=6(35 \%)$ & $\mathrm{n}=8(42 \%)$ \\
\hline 2 & $\mathrm{n}=3(14 \%)$ & $\mathrm{n}=4(16 \%)$ & $\mathrm{n}=3(18 \%)$ & $\mathrm{n}=4(21 \%)$ \\
\hline $2-3$ & $\mathrm{n}=4(18 \%)$ & $\mathrm{n}=3(12 \%)$ & - & - \\
\hline 3 & - & - & - & - \\
\hline $\begin{array}{l}3-4 \\
4 \\
\text { Walking aid }\end{array}$ & $\mathrm{n}=1(4 \%)$ & $\mathrm{n}=3(12 \%)$ & - & - \\
\hline No & $\mathrm{n}=19(86 \%)$ & $\mathrm{n}=22(88 \%)$ & $\mathrm{n}=16(94 \%)$ & $\mathrm{n}=18(95 \%)$ \\
\hline Cain & $\mathrm{n}=2(9 \%)$ & $\mathrm{n}=2(8 \%)$ & $\mathrm{n}=1(6 \%)$ & $\mathrm{n}=1 \quad(5 \%)$ \\
\hline Rollator & $\mathrm{n}=1(5 \%)$ & $\mathrm{n}=1(4 \%)$ & $\mathrm{n}=0$ & $\mathrm{n}=0$ \\
\hline
\end{tabular}

$S D=$ standard deviation $\mid M M S E=$ Mini Mental State Examination $\mid P D=$ Parkinson's disease

Data on the amount of treatment received and compliance with the new therapies are summarised in table three and were similar.

Tab. 3: Summarized data on the amount of treatment received and compliance with the allocated therapies (mental practice verss relaxation).
Control group
Experimental group
Relaxation Mental practice

Time spend with the physiotherapist

- $\quad$ Frequency

- $\quad$ Time in hours

7,6 treatments (SD 3,4)

5,3 hours (SD 1,1)

7,7 treatments (SD 3,6)

5,2 hours (SD 1,3)

Time spend on unguided imagery

Frequency

Time in minutes

Time per session
55 mental practice sessions 271 minutes (SD 257,3) 5 minutes/session mental practice
22 relaxation sessions 268 minutes (SD 449,2) 12 minutes/session relaxation

$\mathrm{SD}=$ standard deviation 
Data provided by the patients in their treatment logs confirmed that therapists delivered the appropriate therapy in each case.

Table four ( $\mathrm{p}$ 163) shows the results from the intention to treat analyses. No significant differences were found between the two groups on any outcome measure at any point.

For the per protocol analysis, seven participants from the experimental and five from the control group were excluded from the entire research population. In ten of these twelve patients the treatment amount was insufficient (below 60\%). One patient from the experimental group was excluded because he used mental practice to relax and one because he did not reach stage 2 of the mental practice framework. The results were similar to the intention to treat analysis (data not shown).

For the sub group analyses, from the entire research population six patients in the mental practice group and five patients in the control group were excluded because they were stage three or higher on the Hoehn and Yahr classification (tab. 2).

Table five ( $\mathrm{p} 155$ ) shows the results from the sub group analysis.

No significant differences were found between the two groups on any outcome measure at any point. However, except for the results of the difference score of the TUG at follow up, all measures showed more average improvement compared to baseline for the mental practice group at both measuring points (calculated in percentages). These differences were not significant. 


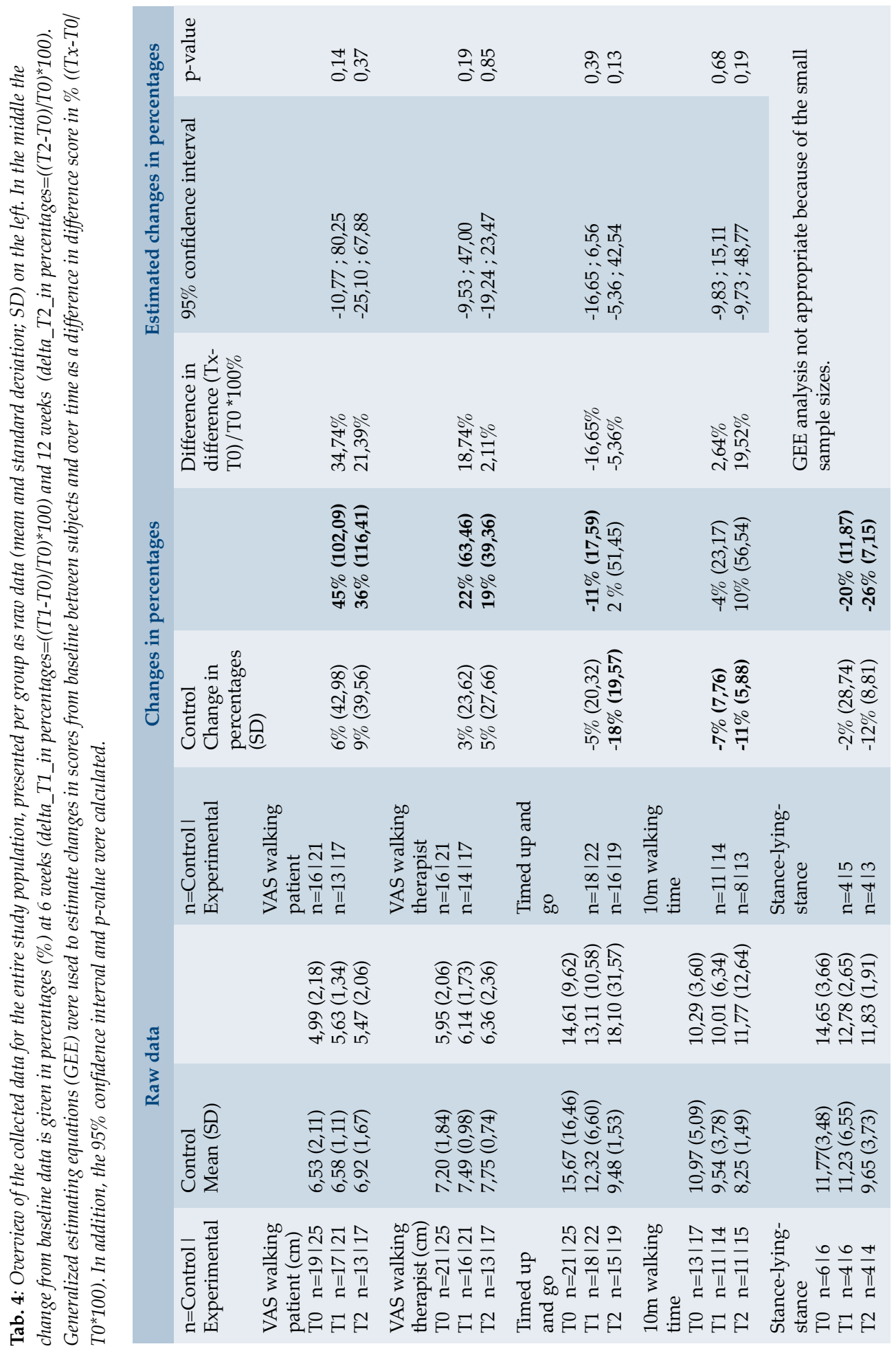




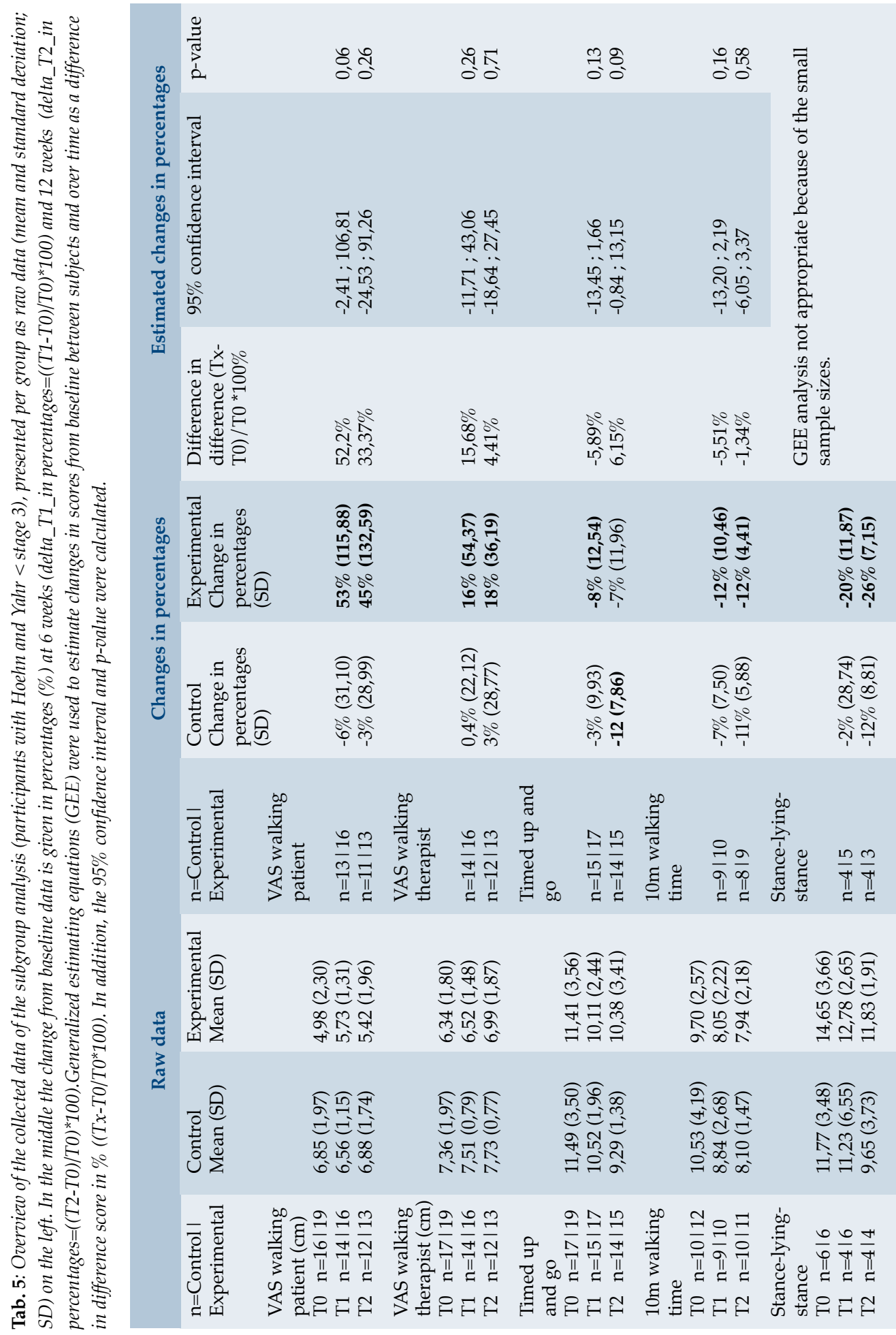




\section{Discussion and conclusions}

In this study, both the intention to treat analysis and the per protocol analysis did not reveal any effects of mental practice on the performance of locomotor task of patients with Parkinson's disease.

In the sub group analysis with only participants with Hoehn and Yahr stages below three, a general trend in favour of the mental practice group was revealed (but was not significant). Why did our study not find a benefit?

\section{Design of the study}

The improvement in both groups in this study was remarkable given that the disease is generally progressive, and that all patients had already received therapy and were still receiving it. One might speculate that both mental practice and relaxation had a beneficial effect. As both groups improved, maybe the contrast between the two interventions was not large enough or the groups were too small to detect possible effects. A control group with an incorporated therapy however was needed to control and compensate for additional attention. Apart from the study by Tamir and co-workers, relaxation has been part of the control intervention in two other studies by Kamsma et al. (1) with significant effects in favour of the experimental treatment. On the other hand, there is also some evidence that relaxation as part of a treatment package might help in patients with Parkinson's disease (1), but at this point there is no evidence, that relaxation as a single intervention is effective to improve locomotor tasks like walking. Effects of both mental practice and relaxation in this study could only have been revealed with a third, only-regulartherapy group, which was not incorporated.

Based on our power calculation, the group sizes had to be sufficient to reveal differences. Perhaps our assumptions were too optimistic or it may have been unrealistic to expect an additional therapy incorporated into an existing treatment program to have the assumed large effect. Therefore the group sizes may have been too small. The study by Tamir and co-workers did reveal significant effects on the SLS and the TUG in a smaller research population $(n=23$; E:12 I C:11) than our total population ( $n=47 ; \mathrm{E}: 25 \mid \mathrm{C}: 22)$. The research population in the study by Tamir and co-workers was quite similar to ours and can therefore not fully explain the difference in results. There were however two main differences in the design: (1) severity of the disease and (2) amount of guided mental practice.

With regard to the first aspect, we included patients with more severe stages of the disease (Hoehn and Yahr stage of 3 and higher), who may have been unable to use the techniques adequately and this might have influenced the results of the entire group. Results from the analysis with the sub group ( $\mathrm{n}=36$; E:19 | C:17), whose patient characteristics were very much like those from the patients from the other RCT, did also not reveal significant effects. 
With regard to the amount of guided mental practice, one might assume, that perhaps patients in our trial did not practice enough under supervision of a physiotherapist. We taught the patients mental practice during a total of six hours therapy instead of 12 in the study by Tamir and coworkers. Partly this is compensated by the unguided imagery in our study. It is however difficult to know to what extent the mental practice therapy was actually used by the patients at home. Some participants reported an additional 15 hours of unguided mental practice, but on average ( 3 hours and 50 minutes) the dose might still have been too small because some participants did not practice unguided at all. On the other hand, if the variety in dose was an important factor in this study, the per protocol analysis should have revealed this (but did not).

\section{Generating evidence for mental practice in patients with Parkinson's disease}

Mental practice is a complex intervention, which is used in patients with a complex pathology. To increase evidence on a new and complex intervention, the Medical Research Council (MRC) (12) advices following research steps: (1) develop theory, explain working mechanism; (2) test feasibility and perform pilot work; (3) full evaluations - effects, costs and process; and (4) implementation. Where does evidence regarding the effects of mental practice in patients with Parkinson's disease stand now?

\section{Develop theory, explain working mechanisms and test feasibility (piloting)}

The working mechanisms for mental practice interventions in Parkinson's disease is based on the evidence from sports and fundamental en clinical research performed over the last 10 years in patients with different pathologies, mainly stroke $(7-10,14)$. The use in patients with Parkinson's disease is primarily based on the idea that it may help as an additional cognitive treatment form in which cueing plays an important role. But as mental practice is a relatively 'new' treatment in patients with Parkinson's disease, it is important to adjust and develop the intervention to the specifics of this population and the individual abilities $(13,23,28,41)$. It seems reasonable to tailor the mental practice content and approach, because it does justice to the way therapists usual work in daily care (clinical decisions are based on individual findings) (7).

In two recent reviews there has been a call for distinction between treatments for moderately and severely affected patients $(1,4)$. Mental practice might well be a treatment only suitable for patients in less severe stages of Parkinson's disease, who are perhaps better at applying the technique. Our sub group analysis and the study of Tamir suggests that this may be the case and this hypothesis is also supported by other literature $(2,25)$.

In addition, patients may also be selected on 'harder' measures regarding their cognitive abilities to engage in mental practice (other than the MMSE). Recently ways of measuring the ability to 
perform imagery, like the hand rotation test and the kinaesthetic visual imagery questionnaire $(15,42,43)$, have been introduced. At this point it is however unknown if being able to perform imagery necessarily equates to benefiting from it in clinical practice. In addition, we presently do not know if people who are unable to perform imagery at baseline, are able to learn it. There are no externally observable signs that can confirm that someone is actually undertaking it during practice. One has to rely on self-report.

Therefore, more research is needed to study underlying mechanisms of why mental practice works in some patients and does not in others. And to predict who is able to perform and benefit from mental practice (selection and prognosis). Within pilot work the mental practice intervention should be tested to determine the optimal content and dose.

\section{Evaluating}

As far as we know, only two small RCTs have been conducted with patients with Parkinson's disease, including this one. No full evaluations of effects, process and costs efficiency of mental practice as an additional therapy form in patients with Parkinson's disease have been undertaken yet. Studies with larger research populations should be conducted. When larger RCTs are undertaken, measuring instruments on different levels should be used and the selection of the instruments should also be based on enabling comparability of research results (1).

From the study by Tamir and co-workers, we could assume, that the higher amount of guided imagery (doses response effect) seems to influence the effect of mental practice positively (25), which becomes an even more important factor considering that unguided imagery is hard to monitor.

Longer term follow-up may also be appropriate because it is possible that the strategy of using mental practice may have a cumulative benefit.

At this point we suggest that there is insufficient evidence to support the routine use of mental practice as performed in this study as part of the rehabilitation package for people with Parkinson's disease (implementation). However it might be helpful, and further research on several levels is warranted $(1,4)$.

\section{Acknowledgments}

We thank all involved therapists and patients for participating in the trial. We appreciate the help of Marieke Spreeuwenberg, MSc, Zuyd University of applied sciences, with the statistical analysis. 


\section{References}

1. Kwakkel G, de Goede CJ, van Wegen EE. Impact of physical therapy for Parkinson's disease: a critical review of the literature. Parkinsonism Relat Disord 2007;13:478-487.

2. Sammer G, Reuter I, Hullmann K, Kaps M, Vaitl D. Training of executive functions in Parkinson's disease. J Neurol Sci 2006;248:115-119.

3. Hoehn MM, Yahr MD. Parkinsonism: onset, progression and mortality. Neurology 1967;17:427-442.

4. Dibble LE, Addison O, Papa E. The effects of exercise on balance in persons with Parkinson's disease: a systematic review across the disability spectrum. J Neurol Phys Ther 2009;33:14-26.

5. Lim I, van Wegen E, de Goede C, et al. Effects of external rhythmical cueing on gait in patients with Parkinson's disease: a systematic review. Clin Rehabil 2005;19:695-713.

6. Nieuwboer A, Baker K, Willems AM, et al. The short-term effects of different cueing modalities on turn speed in people with Parkinson's disease. Neurorehabil Neural Repair 2009;23:831-836.

7. Keus SHJ, Hendriks HJM, Bloem BR, et al. KNGF-Richtlijn 'Parkinson'. Ned Tijdschr Fysiother 2004;114(Suppl):1-86.

8. Feltz D, Landers D. A revised meta-analysis of the mental practise literature on motor skill learning and performance. In: Druckman D. SJ, editors. Enhancing human performance: Issues, theories and techniques. Washington DC: National Academy of Science, 1988. p 1-65.

9. Dickstein R, Deutsch JE. Motor imagery in physical therapist practice. Phys Ther 2007;87: 942-953.

10. Braun SM, Beurskens AJ, Borm PJ, Schack T, Wade DT. The effects of mental practice in stroke rehabilitation: a systematic review. Arch Phys Med Rehabil 2006;87:842-852.

11. Jackson PL, Lafleur MF, Malouin F, Richards C, Doyon J. Potential role of mental practice using motor imagery in neurologic rehabilitation. Arch Phys Med Rehabil 2001;82:1133-1141.

12. Craig P, Dieppe P, Macintyre S, Michie S, Nazareth I, Petticrew M. Developing and evaluating complex interventions: the new Medical Research Council guidance. Bmj 2008;337:a1655.

13. Milton J, Small SL, Solodkin A. Imaging motor imagery: methodological issues related to expertise. Methods 2008;45:336-341.

14. Sharma N, Pomeroy VM, Baron JC. Motor imagery: a backdoor to the motor system after stroke? Stroke 2006;37:1941-1952.

15. Simmons L, Sharma N, Baron JC, Pomeroy VM. Motor imagery to enhance recovery after subcortical stroke: who might benefit, daily dose, and potential effects. Neurorehabil Neural Repair 2008;22:458-467. 
16. Sharma N, Jones PS, Carpenter TA, Baron JC. Mapping the involvement of BA 4a and 4p during Motor Imagery. Neuroimage 2008;41:92-99.

17. Johnson-Frey SH. Stimulation through simulation? Motor imagery and functional reorganization in hemiplegic stroke patients. Brain $\operatorname{Cog} n$ 2004;55:328-331.

18. Johnson SH, Sprehn G, Saykin AJ. Intact motor imagery in chronic upper limb hemiplegics: evidence for activity-independent action representations. J Cogn Neurosci 2002;14:841-852.

19. Cunnington R, Egan GF, O'Sullivan JD, Hughes AJ, Bradshaw JL, Colebatch JG. Motor imagery in Parkinson's disease: a PET study. Mov Disord 2001;16:849-857.

20. Filippi MM, Oliveri M, Pasqualetti P, et al. Effects of motor imagery on motor cortical output topography in Parkinson's disease. Neurology 2001;57:55-61.

21. Frak V, Cohen $\mathrm{H}$, Pourcher E. A dissociation between real and simulated movements in Parkinson's disease. Neuroreport 2004;15:1489-1492.

22. Malouin F, Richards CL. Mental Practice for Relearning Locomotor Skills. Phys Ther 2010;90:240-51.

23. Braun SM, Kleynen M, Schols JM, Schack T, Beurskens AJ, Wade DT. Using mental practice in stroke rehabilitation; a framework. Clin Rehabil 2008;22:579-91.

24. Yaguez L, Canavan AG, Lange HW, Homberg V. Motor learning by imagery is differentially affected in Parkinson's and Huntington's diseases. Behav Brain Res 1999;102:115-127.

25. Tamir R, Dickstein R, Huberman M. Integration of motor imagery and physical practice in group treatment applied to subjects with Parkinson's disease. Neurorehabil Neural Repair 2007;21:68-75.

26. Altman DG, Schulz KF, Moher D, et al. The revised CONSORT statement for reporting randomized trials: explanation and elaboration. Ann Intern Med 2001;134:663-694.

27. Tombaugh TN, McIntyre NJ. The mini-mental state examination: a comprehensive review. J Am Geriatr Soc 1992;40:922-935.

28. Malouin F, Richards CL, Doyon J, Desrosiers J, Belleville S. Training mobility tasks after stroke with combined mental and physical practice: a feasibility study. Neurorehabil Neural Repair 2004;18:66-75.

29. Gessel AH. Edmund Jacobson, M.D., Ph.D.: the founder of scientific relaxation. Int J Psychosom 1989;36:5-14.

30. Budney AJ, Murphy SM, Woolfolk RL. Imagery and Motor performance: What do we really know? In: Sheikh AA, Korn ER, editors. Imagery in Sports and Physical Performance. Farmingdale, NY: Baywood, 1994. p 97-120.

31. Boschker M. Action-Based Imagery. On the nature of mentally imagined motor actions [Dissertation]. Amsterdam: Faculty of Human Movement Sciences, Vrije Universiteit Amsterdam; 2001. 
32. Hall CR, Pongrac J. Movement imagery questionnaire. London, Ontario: The University of Western Ontario; 1983.

33. Wewers ME, Lowe NK. A critical review of visual analogue scales in the measurement of clinical phenomena. Res Nurs Health 1990;13:227-236.

34. Garraway WM, Akhtar AJ, Prescott RJ, Hockey L. Management of acute stroke in the elderly: preliminary results of a controlled trial. Br Med J 1980;280:1040-1043.

35. Morris S, Morris ME, Iansek R. Reliability of measurements obtained with the Timed “Up \& Go" test in people with Parkinson disease. Phys Ther 2001;81:810-818.

36. Hankey GJ, Deleo D, Stewart-Wynne EG. Stroke units: an Australian perspective. Aust N Z J Med 1997;27:437-438.

37. Mathias S, Nayak US, Isaacs B. Balance in elderly patients: the "get-up and go" test. Arch Phys Med Rehabil 1986;67:387-389.

38. Lin MR, Hwang HF, Hu MH, Wu HD, Wang YW, Huang FC. Psychometric comparisons of the timed up and go, one-leg stand, functional reach, and Tinetti balance measures in community-dwelling older people. J Am Geriatr Soc 2004;52:1343-1348.

39. Assmann SF, Pocock SJ, Enos LE, Kasten LE. Subgroup analysis and other (mis)uses of baseline data in clinical trials. Lancet 2000;355:1064-1069.

40. Twisk JWR. Applied longitudinal data analysis for epidemiology. fourth ed. Cambridge: Cambridge university press; 2007.

41. Mulder T, Hochstenbach JB, van Heuvelen MJ, den Otter AR. Motor imagery: the relation between age and imagery capacity. Hum Mov Sci 2007;26:203-211.

42. Parsons LM, Fox PT, Downs JH, et al. Use of implicit motor imagery for visual shape discrimination as revealed by PET. Nature 1995;375:54-58.

43. Malouin F, Richards CL, Jackson PL, Lafleur MF, Durand A, Doyon J. The Kinesthetic and Visual Imagery Questionnaire (KVIQ) for assessing motor imagery in persons with physical disabilities: a reliability and construct validity study. J Neurol Phys Ther 2007;31:20-29. 



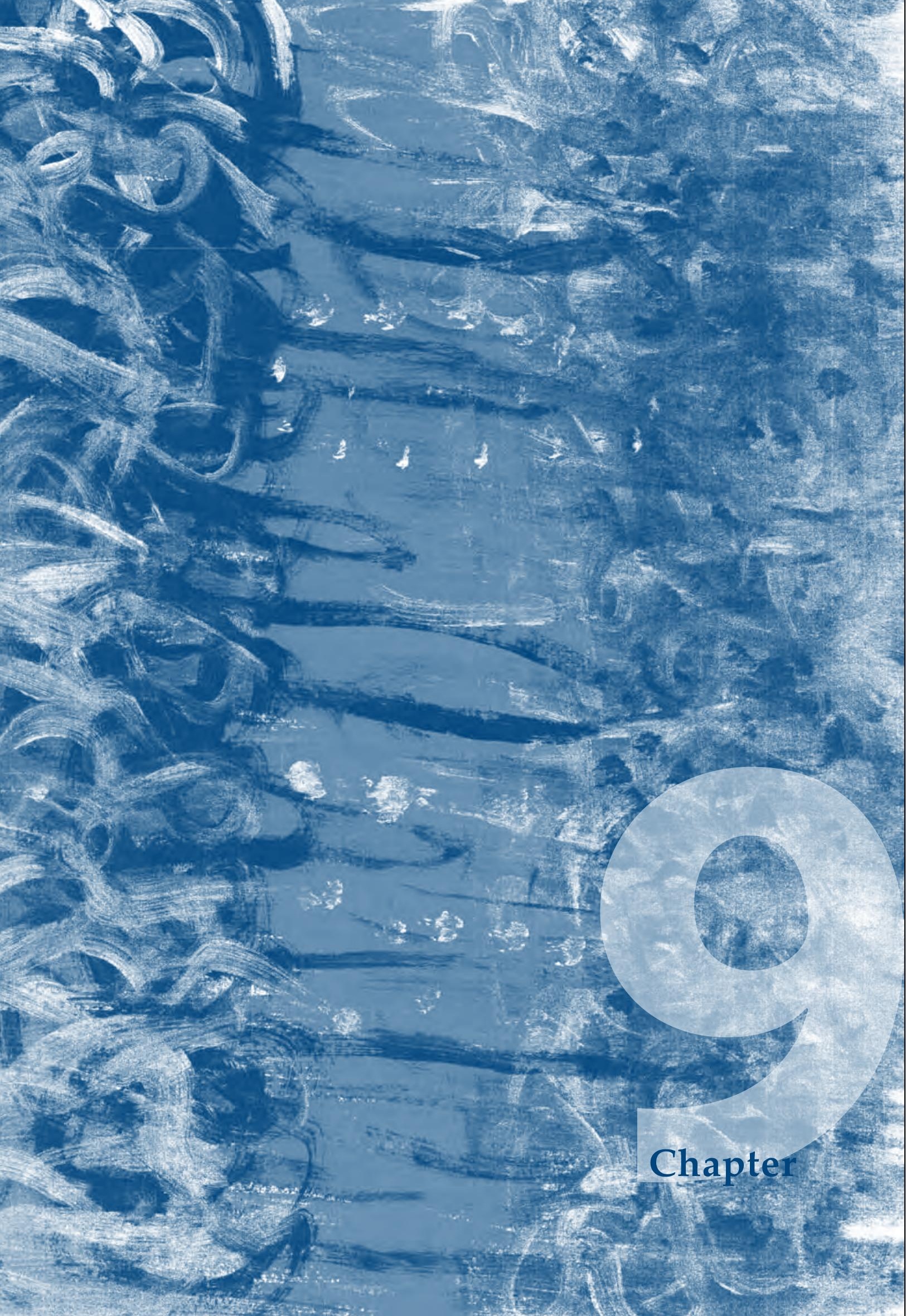




\section{Chapter nine}

The experience of movement imagery therapy by patients with stroke or Parkinson's disease

Braun SM, Beurskens AJ, Dalemans R, Schols JM, Wade DT.

(Submitted 2010) 


\section{Abstract}

Introduction: There is some evidence that mental practice might help in neurological rehabilitation. However, little is known about how neurological patients experience mental practice. Aim of this study was to get insight in the perception of patients with stroke and Parkinson's disease, using a framework to teach and monitor mental practice.

Methods: Seven stroke patients and seven patients with Parkinson's disease were interviewed by independent researchers individually, and five patients with Parkinson's disease through a focus group interview. All participants had six weeks of mental practice experience. A semi-structured interview guide used in sports was adjusted. Patients were recruited until data saturation and results gained through a constant comparison method.

Results: All patients described movement imagery in line with 'practising movements in one thoughts without actually doing it'. Stroke patients seemed more able to apply mental practice than patients with Parkinson's disease. Stroke patients reported three perceived benefits: improving motor skills, changing negative emotions and motivating themselves. Half of the patients with Parkinson's disease perceived benefits only with regard to the stabilisation of motor skills.

Conclusion: The reported responses imply that the patient's experiences and perceptions vary. There were differences between patients after stroke and with Parkinson's disease. Individual perceptions should be considered when using mental practice. 


\section{The experience of movement imagery therapy by patients with stroke or Parkinson's disease}

\section{Introduction}

Movement imagery, usually as part of mental practice, is becoming more popular in neurological rehabilitation, certainly in research with many studies published in the last decade (1-3). Researchers suggest that its use may allow more practice of lost skills by the patient without greater therapy input (4). Movement imagery, the technique itself, is described as 'imagining oneself undertaking the skilled movement without actually doing the movement'. It primarily involves imagining movements by the person, rather than imaging the movement of objects. The subject may imagine the movement in the third (as if looking from a distance at oneself) or the first (as if looking through their own eyes) person's view $(3,4)$. When movement imagery is practiced on a regular basis it is called mental practice: 'During mental practice an internal representation of the movement is activated and the execution of the movement repeatedly mentally simulated within a chosen context. This mental simulation takes place in absence of bodily activity. It is used for the goal-oriented improvement or stabilization of a given movement' (1). In this article, movement imagery is used when the technique of mentally rehearsing a skill is meant. When the emphasis is on the training aspect, using imagery as an additional training method as part of therapy, then we use the term mental practice. When introducing any new therapy it is wise to investigate the experience of patients, especially if it is a complex intervention (5).

On searching the literature we were only able to find one study that reported on patient experience (6), and that was not focusing on the issue. Interestingly there are also few studies of participant-experience of movement imagery even in sports science where the technique has been used for many decades, and qualitative research is only just starting.

The effects of this new therapy are still uncertain (1-3). One reason may be that patients are unwilling or unable to use it. The only way to discover is to explore patient experience. We conducted randomised trials in two groups of patients. The first was in people who have had an acute stroke and are receiving rehabilitation in Dutch nursing homes (7). The other was in people with Parkinson's disease who are living in the community, registered at www.trialregister.nl (NTR 1735).

We have taken this opportunity to research patient experience in these two groups. Our goals were to assess if stroke patients and patients with Parkinson's disease: 
1. can describe movement imagery according to the theoretical concepts taught;

2. can apply mental practice as taught;

3. believe that mental practice:

- is beneficial to their recovery (stroke)

- maintains or improves their motor performance (Parkinson's disease).

We also investigated whether there were differences in perceptions between stroke patients and patients with Parkinson's disease.

\section{Methods}

Semi-structured interviews of participants were part of both trials, which were both approved by the local medical ethical committee. Individual interviews (stroke and Parkinson's disease) as well as one focus group interview (Parkinson's disease only) took place after six weeks of mental practice experience. We used an imagery framework, in which the patient's imagery technique was taught and upgraded in four steps (8). The duration of the different steps and the entire imagery program may vary, depending on the abilities of the patients to learn and use movement imagery. Although different aspects of imagery were explained to the patients and they were stimulated to make their images as vivid as possible (kinesthetic and visual imagery), they were not directed to use only the first or third person's view $(9,10)$. They were allowed to use different kinds of imagery, depending on their personal preferences and the movements they wanted to practice. In figure one it is shown when which activity during the trial took place (next page).

The study with patients after stroke took place in three nursing homes and was separate from the study with patients with Parkinson's disease, which took place in three physiotherapy practices in the community, and included day care patients in one hospital and one nursing home. Even though the two studies ran independently, the study designs and mental practice therapy contents were the same. Stroke patients were recruited two-ten weeks post stroke. Parkinson's disease patients could be in stage I through IV of the Hoehn and Yahr classification and were attending physiotherapy for mobility problems. Other details of the studies are available ((7) and www.trialregister.nl).

Participants were adult stroke survivors and patients with Parkinson's disease who had given additional informed consent for the interviews. The inclusion criteria for the interview was that patients had six weeks of mental practice experience as an additional therapy. Eligible participants for the interviews were contacted by their treating therapist. 


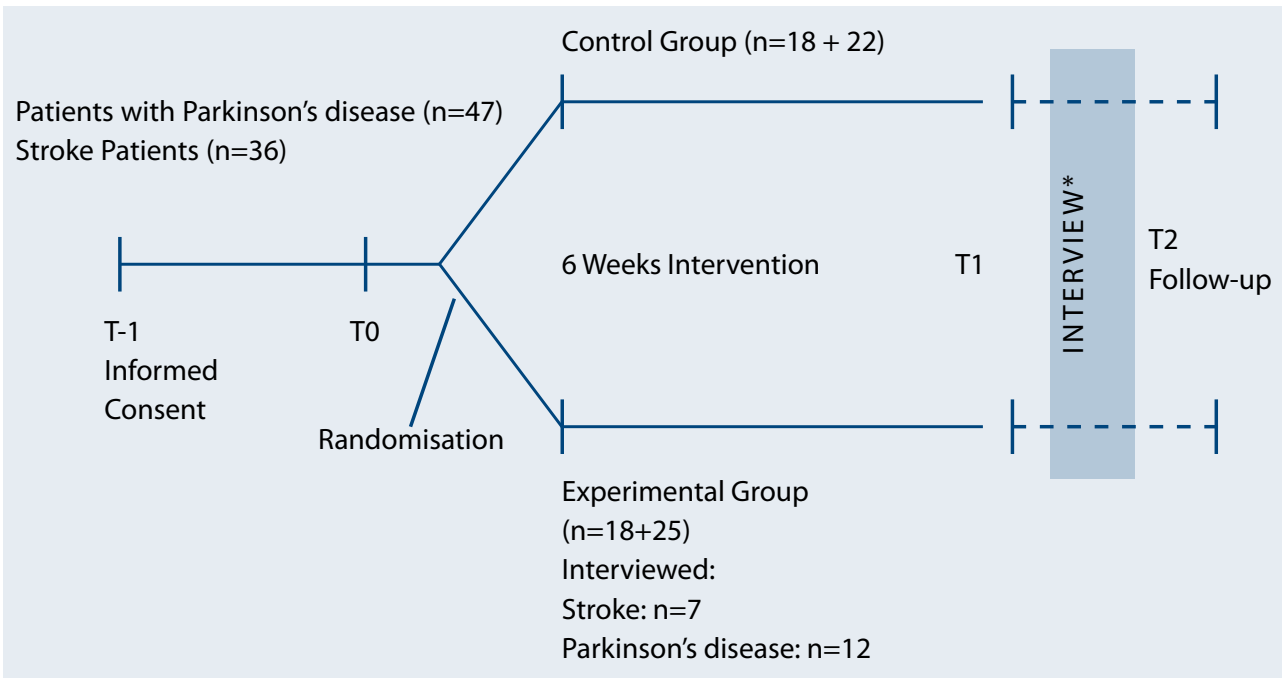

Fig. 1: Overview of the study design and when within the study design the interview took place.

* Interviews took place 2-4 weeks after T1 (ending of the intervention period). To control for attention, a sub sample of both groups were interviewed. The participants of the experimental group were asked about their experiences with mental practice. The participants of the control group were interviewed on how they experienced regular care. In this article only the experiences of the experimental group are presented.

We used purposive sampling. In recruiting of respondents the following characteristics were taken into account: gender, age functional level, localisation of the lesion (stroke) and phase of recovery (stroke). Perception of benefit was not used in selecting patients. Participants for the semi-structured interviews were recruited until no new themes came up in the interviews (data saturation). Apart from the interviews, other data (e.g. on motor performance) were collected as part of the main studies.

\section{Interview guide development}

The interview guide by Driediger and co-workers (15) based on previous work by Monroe and co-workers (16) was used. The guide was translated into Dutch and adjusted for the neurological population. It consisted of three sections:
a) Getting acquainted
b) Questions about movement imagery and mental practice
c) Closing the interview

The protocol was tested in a pilot study by the main researcher for feasibility and validity and was adjusted then and on a continuing basis in response to the answers of the participants: for example when data saturation started, questions on which little or no information was available 
were emphasised. During the focus group interview the questions were written on a flip chart to keep the focus of the group on one questions and to guide the participants through the interview.

\section{Data collection}

Figures two and three give an overview of the data collection, preparation and data analysis for the stroke (fig. 2, next page) and Parkinson's population (fig. 3, p 180). We started with individual interviews. After given informed consent an appointment was made for the interview. They took place in a quiet room at the location they were treated or at their homes, so patients could focus on the questions. An independent researcher, familiar with the mental practice intervention, undertook interviews which were audio taped. Only the interviewer and the patient were present if the interview took place at one of the treating locations. If the interviews were taken at home, sometimes the partner was there as well.

After interviews with five stroke patients and interviews with seven patients with Parkinson's disease, the data were checked with regard to two aspects: (1) the variety in respondents / patient characteristics and (2) saturation of answers or identification of topics to pursue further.

The focus group for people with Parkinson's disease took place at one of the participating practices, in a quiet room. Apart from the independent interviewer, an additional researcher was present and took notes. She also tried to engage all patients in the interview.

\section{Data preparation and data analysis}

The data were analysed by two independent researchers. The researchers met after every analysed interview to compare results. If they disagreed on the results, a third reviewer was approached to reach consensus. After the first interview round the third researcher was also involved to discuss further inclusion of patients and topics for the interviews.

All interviews were transcribed from the audio tape verbatim by the interviewer. Data were organized into electronic file folders. First, the interviews were read in total to get a sense of the whole data before breaking them into parts. Remarks were placed in the margin and redundant information (e.g. interruptions by phone calls, small talk between patient and partner) was removed in the initial process of exploring the data. 


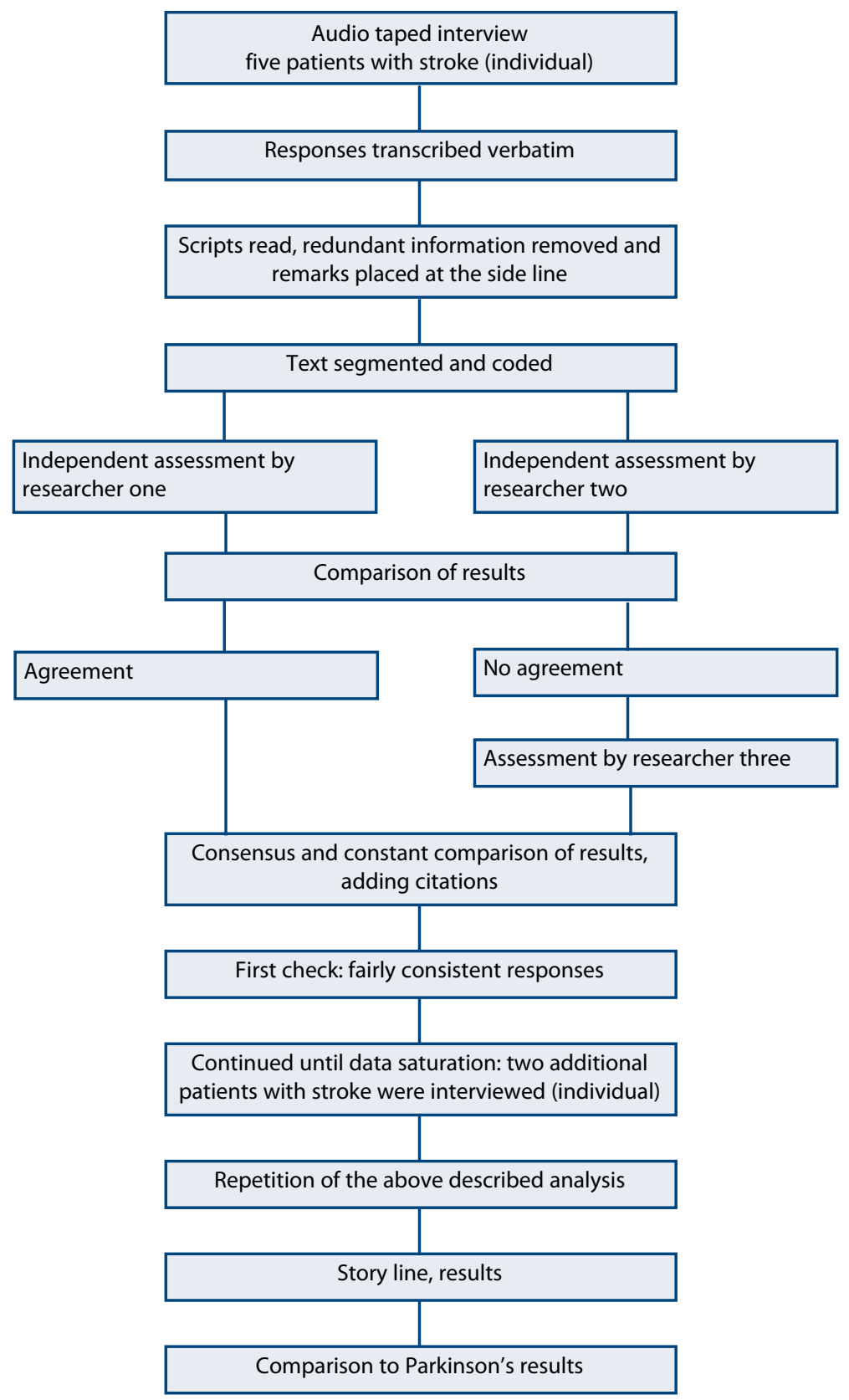

Fig. 2: Overview of the data collection and data analysis procedure for the stroke population. 


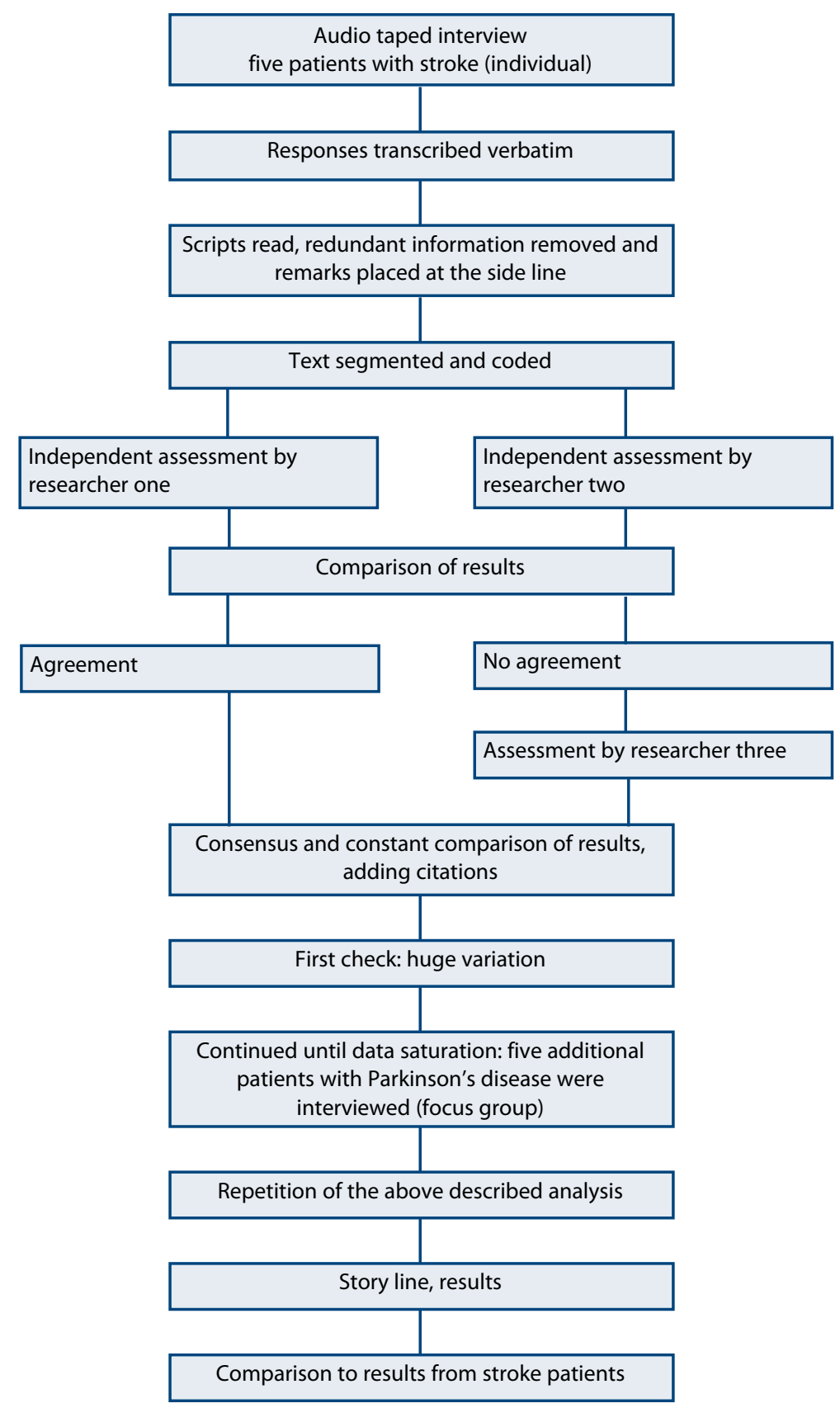

Fig. 3: Overview of the data collection and data analysis procedure for the Parkinson's population. 
Then, the describing, classifying and interpreting loop was entered using a constant comparative method (11). The remaining text was divided into segments, after which an open coding of the segments took place.

The collected codes were put into a priority list. Priority was determined based upon how well the responses could answer the main questions. The number of codes was then reduced by combining similar answers (axial coding) and core categories were defined. Quotations which could illustrate the results of the main questions were noted and labelled to the codes. Mind mapping was used to keep track of the variety of answers (Mind Manager Pro 7 UK, Microsoft).

\section{Results}

Five stroke patients and seven patients with Parkinson's disease gave informed consent for the first cycle of interviews and two stroke patients and five patients with Parkinson disease for the second cycle. In the stroke population only two more individual interviews were scheduled after the first round because answers were already very similar. However, a large variety of answers was seen in the Parkinson's disease population after the first check. Therefore, a focus group interview was organized. This way more responses could be collected within one interview session.

Time for one interview ranged from 40 to 90 minutes. Table one (next page) gives an overview of the patients' characteristics and how these were balanced across the participants.

Results will be presented in the text below for each group, the stroke population first followed by results of the Parkinson's disease population. Table two ( $p$ 176) gives an overview of the main results with regard to the responses, described per neurological condition: description of movement imagery, what, where, when and why mental practice was used. At the end, similarities and differences between groups will be described.

\section{Stroke}

The descriptions of mental practice from all patients were very similar, encapsulated thus: 'You already have in your head stored what you are supposed to do'.

Almost all patients reported using mental practice for many different daily activities, like walking, standing up from a chair and climbing stairs. Some patients had a specific goal, like relearning to write a signature. All patients reported being able to visualize the movements they wanted to improve, mostly from a first person's perspective. Even though they stated they saw themselves 


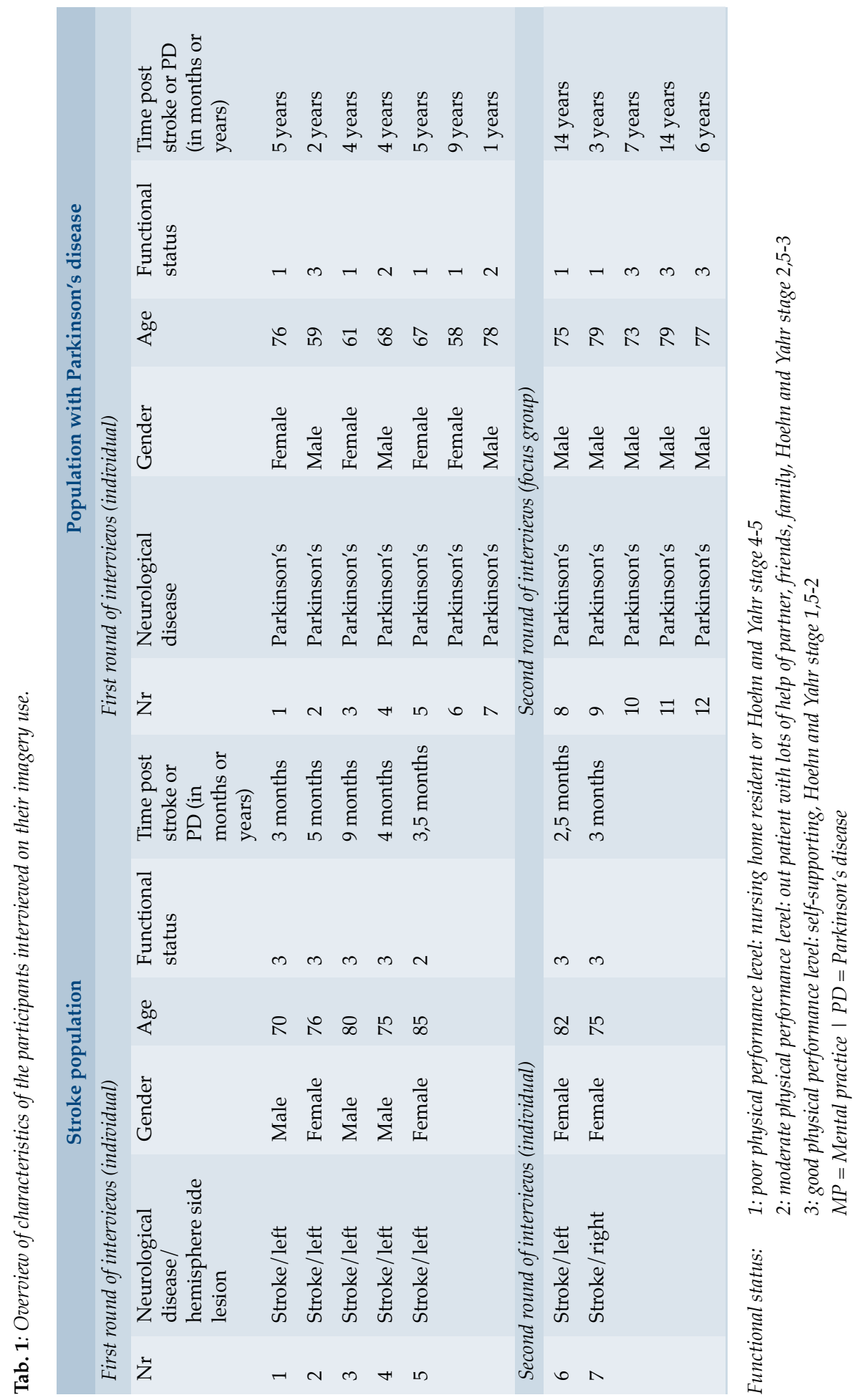


as if looking through their own eyes, descriptions only covered aspects of visual imagery. None were able to generate any kinesthetic feeling during imagery. Most were aware that they were not able to feel anything but were thinking the feeling into the picture: 'Well, I am thinking the feeling, since I am not able to feel it. The feelings, I think them, but I don't actually feel them when I am thinking'.

Mental practice sessions were generally reported to be short, varying from a few seconds to 15 seconds. One patient replied that the length of a visualisation depended on how difficult the movement was to perform. Some patients used mental practice a few times a day whereas others used imagery almost constantly. 'Well, I actually use it all the time, all the time'.

Most patients practiced mentally more during therapy than outside of therapy, and most applied mental practice more frequently before actually performing the skill. The main other location where patients practiced was in bed, either before going to sleep, when not being able to sleep at night, or before standing up 'And even at night, when I woke up, I would think about how I could walk again'. Sometimes the imagery activities were combined with observing other patients or visitors. 'For instance, when I am sitting somewhere and I see people walking. [...] and how I should make the steps in order to be able to walk like that'.

Patients reported three domains in which they perceived benefits by use of mental practice: improving motor skills, motivating themselves for therapy, and altering negative cognition or emotions. Apart from reporting physical benefits, most patients also reported that focusing on the movement, rehearsing the movement in their heads, gave them a secure or less frightened feeling. 'When I have practiced climbing the stairs mentally, I know exactly what I am about to do physically. This makes me feel safe'. Another respondent replied 'It [movement imagery] creates security, that you are not frightened'. Some of the stroke patients reported using imagery to motivate themselves to perform skills or to remain motivated for therapy. 'It [imagery] reinforces my desire to get better'.

\section{Parkinson's disease patients}

The descriptions of mental practice from patients with Parkinson's disease varied. Some patients described mental practice as 'first walking in thought, then in real' or 'preparing mentally on what is to come'. Another patient described mental practice as 'activating the imagery ability to cope with problems you encounter every day', whereas another patient emphasized 'Visualizing how you would walk if you didn't have it [Parkinson's disease]'.

Three participants were unable to visualise themselves. Those who were able to visualize themselves replied that imagery was only possible through great mental effort. Descriptions 
Tab. 2: Overview of the main results of the research for all patients and in comparison (stroke versus Parkinson's disease), with regard to the descriptions, what, where, when and why patients use mental practice.

\begin{tabular}{|c|c|c|}
\hline & Stroke & Parkinson's disease \\
\hline $\begin{array}{l}\text { Description } \\
\text { of movement } \\
\text { imagery }\end{array}$ & $\begin{array}{l}\sqrt{ } \text { Thinking about what you are going } \\
\text { to do } \\
\sqrt{ } \text { Going through the movement in } \\
\text { your mind } \\
\sqrt{ } \text { Chop up what you are planning to } \\
\text { do in little pieces. }\end{array}$ & $\begin{array}{l}\sqrt{ } \text { First walking in thought, then in real } \\
\sqrt{ } \text { Focussing beforehand on what you are } \\
\text { going to do } \\
\sqrt{ } \text { Visualize how you walk if you were not } \\
\text { impaired } \\
\sqrt{ } \text { Imagine how it should be } \\
\sqrt{ } \text { Thinking how to do something, so it } \\
\text { becomes an automatism } \\
\sqrt{ } \text { Activating your ability to imagine, } \\
\text { focussed on the problems you encounter } \\
\sqrt{ } \text { Chop up what you are planning to do in } \\
\text { little pieces. } \\
\sqrt{ } \text { Preparing mentally on what you are about } \\
\text { to do } \\
\text { (No response from two participants from the } \\
\text { focus group interview and one participant } \\
\text { replied he did not know). }\end{array}$ \\
\hline What & $\begin{array}{l}\sqrt{ } \text { Daily activities, walking, standing up } \\
\text { from a chair, climbing stairs } \\
\sqrt{ } \text { Mainly first person's view } \\
\sqrt{ } \text { Only visualisation } \\
\sqrt{ } \text { Short sessions, few to } 15 \text { seconds per } \\
\text { visualization }\end{array}$ & $\begin{array}{l}\sqrt{ } \text { Daily activities and sports, hobbies } \\
\sqrt{ } \text { Mainly third person's view; side, front, } \\
\text { back: only the surroundings } \\
\sqrt{ } \text { Mainly visualisation, some kinesthetic } \\
\sqrt{ } \text { Eyes closed }\end{array}$ \\
\hline Where & $\begin{array}{l}\sqrt{ } \text { In bed, in the corridors / watching } \\
\text { others }\end{array}$ & $\sqrt{ }$ Sitting, any location, quiet environment \\
\hline When & $\begin{array}{l}\sqrt{ } \text { Guided more than unguided } \\
\sqrt{ } \text { A couple of times a day up to all the } \\
\text { time } \\
\sqrt{ } \text { Before performing an activity }\end{array}$ & $\begin{array}{l}\sqrt{ } \text { Guided more than unguided } \\
\sqrt{ } \text { Not up to several times a day }\end{array}$ \\
\hline $\begin{array}{l}\text { Why / } \\
\text { benefits }\end{array}$ & $\begin{array}{l}\sqrt{ } \text { Motor recovery } \\
\sqrt{ } \text { Increase motivation for therapy } \\
\sqrt{ } \text { Influence negative emotions, e.g. fear } \\
\text { or increase positive feeling, e.g. self } \\
\text { confidence }\end{array}$ & $\sqrt{ }$ Movement stabilization/improvement \\
\hline
\end{tabular}


mainly covered aspects of visual imagery or symbolic rehearsal (e.g. recall 'words' in order to practice a strategy). Lack of concentration, fatigue and distracting surroundings were mentioned as main reasons for failure. 'At some point I went on a holiday and my therapist said to me I could practice mentally on the train or at the hotel. I tried, but if you are not used to travelling by train you do not know what to expect. It was quite bumpy and I just could not do it [practice mentally]. It was way to distracting for me and I could just not keep focused'.

Those patients who could visualise 'saw' themselves from a first or third point of view, from the front, back or side. A few respondents saw the room or surroundings they were practising in mentally. Some patients visualized themselves moving with their limitations, others as if they were not affected by Parkinson's. 'When I imagine that I am walking, I still walk normal. This is different from when I actually walk. I can feel the difference'. Two patients reported kinesthetic sensations during imagery with regard to their posture.

The main skill patients with Parkinson's disease wanted to improve was walking. Most participants focused on the entire walk, some used mental practice to improve part, like lifting their feet or swinging their arms more. 'When I imagine that I am walking, I still walk normal. This is different from when I actually walk. I can feel the difference'. Other activities that were mentioned were: standing up from a chair, turning in bed, picking something up from the floor, putting on socks and Nordic walking. 'We don't all have the same skills and exercises. We have lots of exercises. I for instance use mental practice for walking, but walking in Nordic walking. You need to make long steps, the longer the step, the more you get a floating feeling. When I am thinking about Nordic walking, this floating, gliding feeling automatically comes'. Some patients thought that mental practice was not useful or probably not effective. 'Oh no, I just did it because I was asked' or 'I did not get my hopes up too high: you can only get disappointed'.

Most patients tried to practice mentally outside of therapy, but reported that mental practice was more successful if they were guided or instructed by the therapist. Some patients reported being able to practice in different locations (e.g. at home, in bed, in a chair) and they reported more frequent use (six-seven times a day). Most patients preferred a quiet environment, usually the same one and they reported less frequent practice. 'When I am sitting on this specific chair in that room, then I know exactly what to do when I am walking and moving and using my arm.'

Not all patients with Parkinson's disease reported benefits, and those reported were mostly on motor performance. One respondent specifically referred to a tricky situation in the bathroom: the wet part of the shower cabin after taking a shower. 'I used to pull my leg after me, but my therapist 
taught me to shift my weight to the other leg and lift my affected one. At first I could not do it but by practising mentally, lately I can. And that is what I mean with first practising in your head and then doing it'. Another respondent used imagery to determine the best route for instance in a crowded room at a party to get from one point to another. Another used mental practice to try out different strategies in complex situations, like vacuuming a large living room.

None of the patients with Parkinson's disease reported using mental practice for motivational reasons or to alter emotions or cognition.

\section{The contrast - stroke and Parkinson's disease}

Apart from differences in the description of movement imagery, the main difference between the neurological conditions was in the application of mental practice, which seemed to be more difficult for the patients with Parkinson's disease than for patients suffering from stroke. The other main difference was that stroke patients additionally used mental practice to feel more confident and to motivate themselves for therapy.

\section{Discussion}

Both stroke patients and patients with Parkinson's disease could describe mental practice according to the theoretical concept, 'practising a movement in thought without actually doing it'. All described mainly the visual aspects of imagery in contrast to motor imagery, even if they visualized in the first person's view (stroke) (12). A shift from first to third view which is seen in elderly was not generally found in this study (10). Stroke patients seemed to be more unanimous about what mental practice is in contrast to the various descriptions of movement imagery given by patients with Parkinson's disease. Of three participants in the Parkinson's group no description of movement imagery was either reported (two), or the participant was unable to describe movement imagery. The specific reason for the latter is unclear.

In patients with Parkinson's disease, getting the idea theoretically and learning to apply mental practice seemed to be two different things. Three patients with Parkinson's disease were unable to image at all even though they could describe the phenomenon. Yaguez and co-workers reported that some patients with Parkinson's disease lack the ability to image movement because of their disease (13). On the other hand, in a study by Heremans and co-workers, 20 out of 21 patients with Parkinson's disease were able to perform imagery as determined by their recorded eye movements (14). A part of the difference in results may be explained by the fact that generating an image once in laboratory circumstances is different from repeatedly applying imagery in a clinical setting as therapy (more noise). Reported barriers were lack of concentration, fatigue and distracting surroundings. 
A problem may also have been the selection of the eligible participants. Most inclusion criteria were based on clinical judgements. After start of the trials several papers were published in which assessment of the ability to perform imagery more objectively were reported which would certainly be interesting to use in future research $(6,15,16)$. Possibly such measures identify patients who actually can perform imagery. On the other hand, some patients were tested with the hand-rotation-test after the interviews (20), but results were not conclusive. Finally, it is probable that some patients who can use imagery do not want to use imagery. For some patients the intervention type may not have been appealing (reported by some patients).

Both neurological populations reported being facilitated in using imagery when they were guided by their therapist during practice, but patients with Parkinson's disease seemed to need more facilitation. All stroke patients and half of the patients with Parkinson's disease thought that they could benefit from mental practice and intended to use it in the future as an additional skill to improve performance.

In line with the reports from Simmons and co-workers all stroke patients were positive about mental practice (6). Apart from possible benefits mental practice could have on motor recovery almost all stroke patients used mental practice also to motivate themselves for therapy or to alter negative emotions (e.g. fear, anxiety, low self confidence). 'To feel secure' has been reported before as being an important aspect of quality of life in nursing home residents (17) and mental practice seemed to contribute to this feeling of security in the stroke patients in this study. The use of mental practice for these domains has been reported extensively in healthy and injured athletes (18-21), but not yet in patients with neurological conditions.

Patients with Parkinson's disease were less optimistic about possible benefits. Reasons for this difference could lie in the areas of the brain affected by Parkinson's disease, or in the progressive nature of the disease, or in other disease-specific factors. Being able to recover might have been motivational for stroke patients to put effort into 'new' therapy ideas and to gain as much recovery as possible. Patients with Parkinson's disease face a completely different future in which decrease of function, not recovery is reality.

Another reason for differences in perception between the two groups could be that the stroke patients in this study seemed to have in average a higher level of physical performance than the patients with Parkinson's disease. Particularly pertinent could be that motor imagery may be associated with actual physical ability (22). In sports, coaches seem to stimulate athletes on a higher level of performance more than recreational athletes to use imagery. Maybe this also 
accounts for therapists: perhaps they motivate patients with a higher performance level more than patients that cannot perform that well. Probably the cognitive abilities of the patient with severe physical disabilities play an important role as well. Generally, imagery use in athletes is very much determined by how much the coaches motivate them to use imagery (23).

\section{Methodological quality of the study}

To identify the preferences and abilities of the patients, a qualitative approach was used. We decided to use semi-structured interviews for two reasons. First, they leave more opportunity to rephrase or refine answers. New topics can emerge and be explored. Second, in interviews, the interviewer is able to cope with possible effects neurological diseases can have on problems with understanding and replying to answers. The researcher can adjust to the individual capacities of the respondent and retrieve the information needed.

In order to gain more information on specific topics in a limited amount of time, a focus group interview was conducted in the Parkinson's population. The main advantage was that more information on the missing data was collected in a very short time span. A disadvantage was that not all of the five patients responded to all questions. In addition, the questions could not always be tailored to the individual abilities of the patients. Nonetheless, the information gathered from the focus group interview was complementary to the individual ones.

In both neurological conditions data were collected until saturation and all predefined participants' characteristics were included in the sample. In the patients with Parkinson's disease the characteristics were more balanced than in the stroke population (especially localisation lesion side). Although the patients were representative of the populations in these settings, not the entire variation could be captured. The results should therefore be interpreted with care.

\section{Practical implications}

In general, motivation of the patient to use imagery may be increased by structural guided imagery together with the therapist. The treating therapist therefore has an essential and continuous role in the potential effects mental practice might have (23). Monitoring and improving imagery skills should not only be emphasized at the beginning of the framework but should remain an important part of the intervention throughout therapy. Moreover, it seems important to check continuously what patients are thinking. In this study, stroke patients altered their goals of mental practice during the trial. These effects may not have been noticed or measured if patients were not asked. 
Apart from differences in neurological populations, individual preferences seem to be important as well: there were individual patients with Parkinson's disease who were positive about the intervention and stroke patients who were more careful in their responses.

Furthermore, the present condition of a patient will influence the applicability and the chance of possible effects of mental practice in therapy $(24,25)$. The status quo will also for instance determine the chosen skills or to which extend patients can recall their way of moving before the disease onset.

These practical implications should be considered with caution, because some of the patients may not have been able to perform movement imagery.

\section{References}

1. Braun SM, Beurskens AJ, Borm PJ, Schack T, Wade DT. The effects of mental practice in stroke rehabilitation: a systematic review. Arch Phys Med Rehabil 2006;87:842-52.

2. Dickstein R, Deutsch JE. Motor imagery in physical therapist practice. Phys Ther 2007;87:94253.

3. Sharma N, Pomeroy VM, Baron JC. Motor imagery: a backdoor to the motor system after stroke? Stroke 2006;37:1941-52.

4. Jackson PL, Lafleur MF, Malouin F, Richards C, Doyon J. Potential role of mental practice using motor imagery in neurologic rehabilitation. Arch Phys Med Rehabil 2001;82:1133-41.

5. Craig P, Dieppe P, Macintyre S, Michie S, Nazareth I, Petticrew M. Developing and evaluating complex interventions: the new Medical Research Council guidance. BMJ 2008;337:a1655.

6. Simmons L, Sharma N, Baron JC, Pomeroy VM. Motor imagery to enhance recovery after subcortical stroke: who might benefit, daily dose, and potential effects. Neurorehabil Neural Repair 2008;22:458-67.

7. Braun SM, Beurskens AJ, van Kroonenburgh SM, Demarteau J, Schols JM, Wade DT. Effects of mental practice embedded in daily therapy compared to therapy as usual in adult stroke patients in Dutch nursing homes: design of a randomised controlled trial. BMC Neurol 2007;7:34.

8. Braun S, Kleynen M, Schols J, Schack T, Beurskens A, Wade D. Using mental practice in stroke rehabilitation: a framework. Clin Rehabil 2008;22:579-91.

9. Malouin F, Richards CL, Jackson PL, Lafleur MF, Durand A, Doyon J. The Kinesthetic and Visual Imagery Questionnaire (KVIQ) for assessing motor imagery in persons with physical disabilities: a reliability and construct validity study. J Neurol Phys Ther 2007;31:20-9. 
10. Mulder T, Hochstenbach JB, van Heuvelen MJ, den Otter AR. Motor imagery: the relation between age and imagery capacity. Hum Mov Sci 2007;26:203-11.

11. Patton MQ. Qualitative research and evaluation methods. Sage Publications Ltd., London; 2002.

12. Mattingley JB, Driver J, Beschin N, Robertson IH. Attentional competition between modalities: extinction between touch and vision after right hemisphere damage. Neuropsychologia 1997;35:867-80.

13. Yaguez L, Canavan AG, Lange HW, Homberg V. Motor learning by imagery is differentially affected in Parkinson's and Huntington's diseases. Behav Brain Res 1999;102:115-27.

14. Heremans E. The effects of external cueing on motor imagery in healthy persons and patients with Parkinson's disease. Third Heerlen - Oxford conference on imagery and neurological rehabilitation. 2009, May 14th; Heerlen. p 4.

15. Malouin F, Richards CL, Durand A, Doyon J. Clinical assessment of motor imagery after stroke. Neurorehabil Neural Repair 2008;22:330-40.

16. Malouin F, Richards CL, Durand A, Doyon J. Reliability of mental chronometry for assessing motor imagery ability after stroke. Arch Phys Med Rehabil 2008;89:311-9.

17. Hjaltadottir I, Gustafsdottir M. Quality of life in nursing homes: perception of physically frail elderly residents. Scand J Caring Sci 2007;21:48-55.

18. Driediger M, Hall C, Callow N. Imagery use by injured athletes: a qualitative analysis. Journal of sports sciences 2006;24:261-71.

19. Evans L, Hare R, Mullen R. Imagery use during rehabilitation from injury. Journal of imagery research in sport and physical activity 2006;1:1-19.

20. MacIntyre TE, Moran AP. A qualitative investigation of imagery use and meta-imagery processes among elite canoe-slalom competitors. Journal of imagery research in sport and physical activity 2007;2:1-19.

21. Martin K, Moritz S, Hall C. Imagery use in sports: a literature review and applied model. The Sports Psychologist 1999;13:245-68.

22. Milton J, Small SL, Solodkin A. Imaging motor imagery: methodological issues related to expertise. Methods 2008;45:336-41.

23. Jedlic B, Hall N, Munroe-Chandler K, Hall C. Coaches' encouragement of athletes' imagery use. Res Q Exerc Sport 2007;78:351-63.

24. Sackett DL, Scott Richardson W, Rosenberg W, Haynes BB. Evidence-based medicine: how to practice and teach EBM. Churchill Livingstone, New York; 1997.

25. Sackett DL, Rosenberg WM, Gray JA, Haynes RB, Richardson WS. Evidence based medicine: what it is and what it isn't. Bmj 1996;312:71-2. 

In

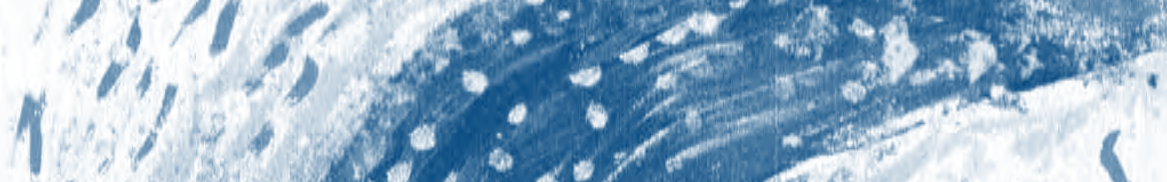
- 1 - $19+20: 0$

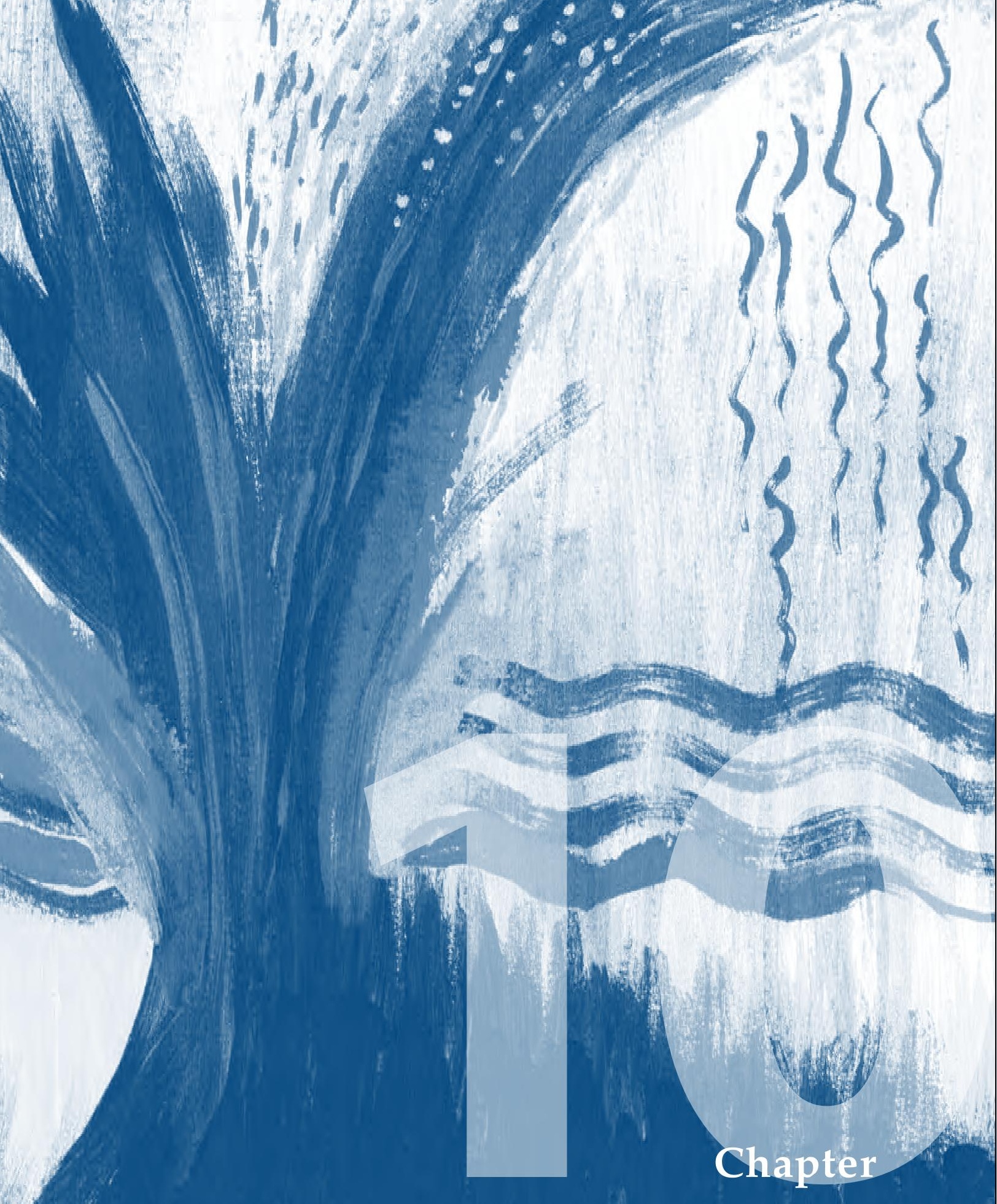




\section{Chapter 10}

\section{Developing and evaluating movement imagery and mental practice}

In this research project we assessed the potential use of movement imagery and mental practice for learning daily activities in patients after stroke and in patients with Parkinson's disease. The study was particularly difficult because it was investigating a complex intervention, which was taught to patients with complex pathologies and who were managed within a complex system of differing and variable clinical pathways. As stated in the introduction, we structured the research project according to the cycle process of developing and evaluating complex interventions as suggested by the Medical Research Council (MRC, fig. 1) (1).

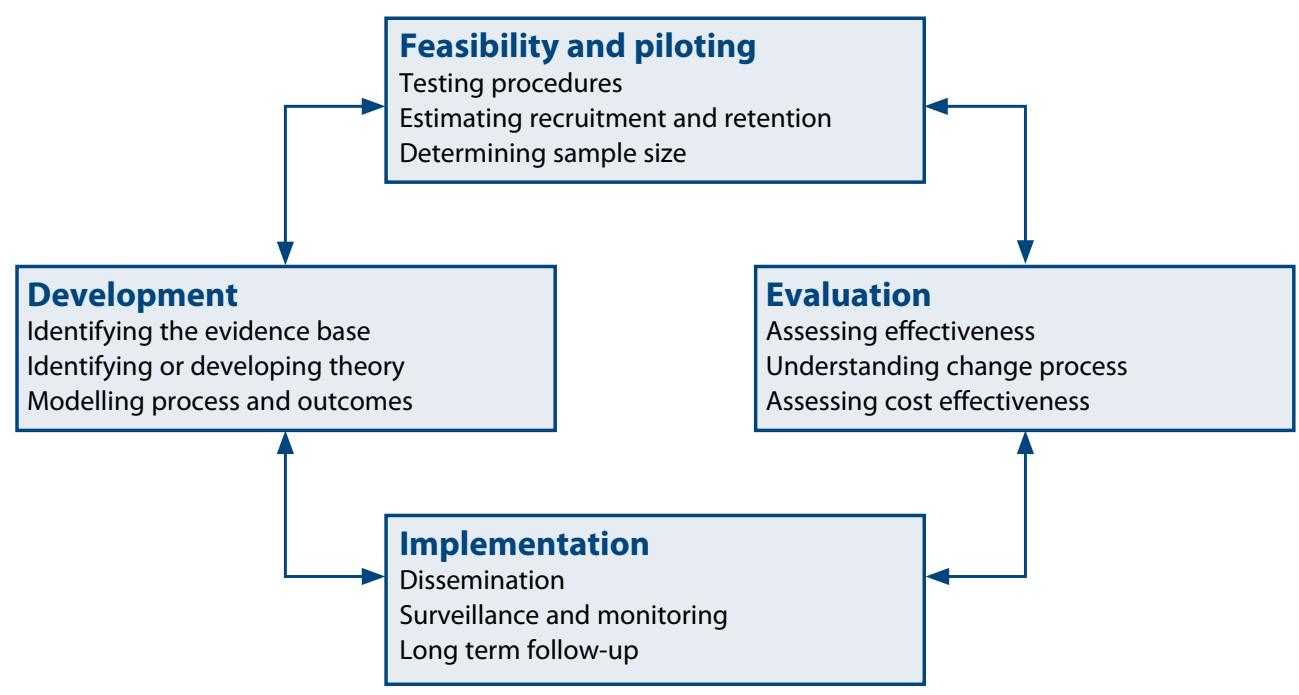

Fig. 1: Key elements of the development and evaluation process (model of the MRC guide (1)).

Several stages or components are distinguished in the process (fig. 1):

1. Development: reviewing of, or contributing to the background theory, in this instance exploring the field of mental practice (e.g. ability to image movements) and modelling mental practice interventions;

2. Feasibility and piloting: testing the mental practice intervention and the measures in routine care; 
3. Evaluation: applying mental practice in clinical research and evaluating the patients' and therapists' opinion of the intervention, evaluating the processes of treatment, and acquiring evidence on its effects (good and bad);

4. Implementation: possibly implementing mental practice in daily care.

We went through (parts of) this cycle twice: almost the entire cycle was used for the development and evaluation of mental practice in stroke patients and parts were used in patients with Parkinson's disease. Figure two shows the same steps as reported in the MRC guide, but the figure is now restructured, emphasising the particular content of the research undertaken in this project.

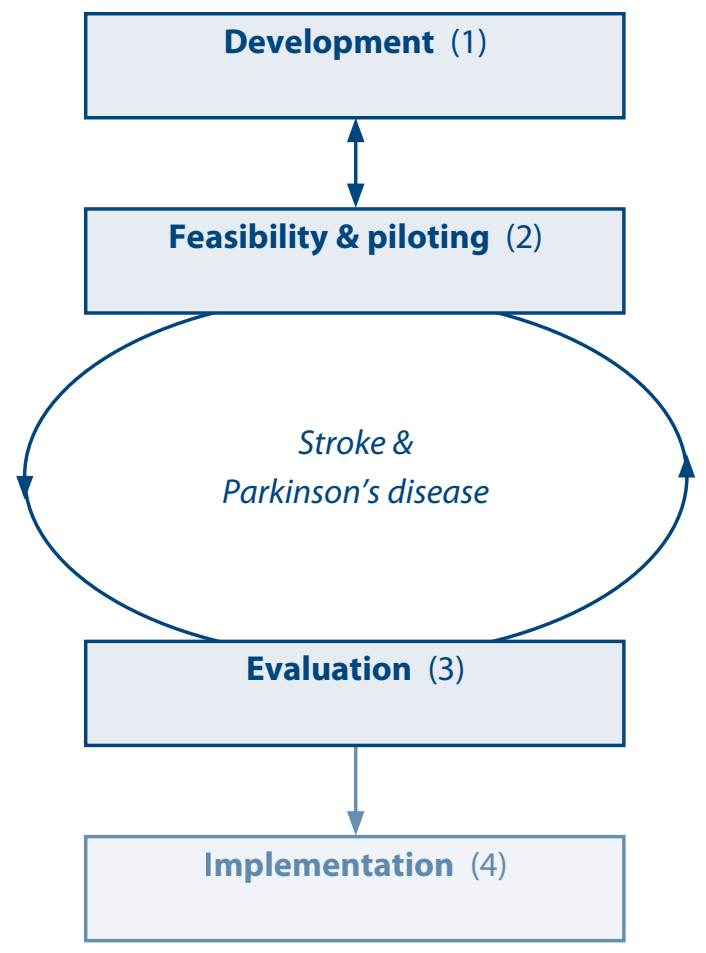

Fig. 2: Restructured figure of the key elements of the development and evaluation process of the MRC (1), with focus on the three main elements in this research project: development, feasibility and piloting and evaluation.

Although we prepared our trials carefully, the results of both the stroke and Parkinson's trial were disappointing: both groups improved, but there was no significant difference in outcome on any outcome measure at any of the measuring points in favour of the mental practice intervention. There were some promising results in a sub group of Parkinson's patients, but groups were too small to reveal significant improvement compared to the control group. 
There was not enough evidence to recommend implementation of the developed framework into routine care.

Almost all research on mental practice in neurological populations reported so far has given positive results. Why did our research not support previous findings? Why could we not translate the positive outcome from fundamental research and earlier studies (steps 1 and 2) into positive trials and recommend implementation into routine care (steps 3 and 4)?

There are several possible explanations that relate well to the key elements of the MRC, and we will use the MRC framework to discuss our study results. Ongoing research by others has also shed new light on the topics and questions raised from our own studies will be discussed in the context of results from the literature and other recent or ongoing studies:

1. Development:

- Does the ability to image automatically imply that neurological patients can and will apply mental practice?

- Is there less evidence for effects of mental practice than we think there is?

- Should mental practice content be fixed or adjusted to personal preferences, stage of motor learning or skill expertise?

\section{Feasibility and piloting:}

- Does preliminary work lead to the preparation of trials?

3. Evaluation:

- Have full evaluations (effect, cost and process) of mental practice interventions within clinical trials in neurological conditions taken place?

- Where does ongoing research in other pathologies stand?

\section{Implementation:}

- Where should we go from here?

And finally, the general discussion ends on a more personal note by emphasising what this research project has meant for so many more people than we could have imagined when we started five years ago.

\section{Developmental work within our research}

Results from two research areas have been important at the start of the project. First, the working mechanism for mental practice in neurological conditions was based on positive results from brain imaging studies $(2,3)$, leading to the hypothesis that it might have a potential role in neurological rehabilitation $(4,5)$. Second, results from mental practice interventions and mental 
practice approaches in sports were the basis for our clinical work $(6,7)$. In the development stage, three steps were undertaken. First, we explored the potential use of an existing computer program (SDA-M) for visualizing mental representations of motor actions. Secondly we undertook a systematic review to identify the evidence base for effects of mental practice in rehabilitation. Third, we developed a defined, specific and described mental practice intervention, which few other studies have done.

We explored whether it was possible to know what patients were visualizing and feeling during movement imagery. When we started, the most frequently used questionnaire was the Vividness of Movement Imagery Questionnaire (VMIQ (8)) which we could not use, because the questions asked are difficult or inappropriate for patients with stroke (e.g. jumping, running, kicking a ball).

The Structural Dimension Analysis of Motor memory (SDA-M) had been used in healthy subjects and athletes (9). It seemed feasible in stroke patients but assessment was time consuming and little is known about the methodological quality (e.g. validity is based on construct and expert opinion, chapter three (10)). The interpretation of the tree diagrams was usable in clinical practice when analyzing a single patient's performance, but the results from the SDA-M where hard to quantify as an outcome measure. Comparison with other measures was not possible.

Apart from the SDA-M, the ability to use mental practice was based on a combination of the score on the Mini Mental State Examination (MMSE score (11)) and the clinical judgment of the treating therapist (and sometimes the psychologist and doctor) of each patient's ability to engage in mental practice.

In 2005, 10 articles on mental practice in stroke rehabilitation were included in our review (chapter two (12)). In nine studies mental practice lead to a significant improvement. The mental practice treatments were less complex (e.g. tape recording) than our framework in eight studies. No training of therapists was reported. No sub groups within the stroke population seemed to benefit more from mental practice and no treatment content or regimes seemed to work better.

When this study started, less research had been done in patients with Parkinson's disease compared to the stroke population: two small experimental trials and four brain imaging studies (e.g. $(13,14))$. In 2007, a larger clinical trial with positive effects appeared (15). Again, there was no detailed description of the intervention in these trials.

Motor learning in neurological patients is in principle not different from motor learning in healthy subjects and athletes (16). This is confirmed by the National guidelines for stroke patients and 
patients with Parkinson's disease $(17,18)$ : goal-oriented movements need to be practiced in context specific situation and preferably as much as possible (19-21). The more a patient practices within the physiological boundaries, the more is gained. This led to the conclusion that the mental practice intervention could not possibly be a fixed program $(1,22)$, but needed sufficient opportunity to adjust mental practice to the patients' abilities and preferences (22) (Chapter four (23)).

We therefore developed a framework within which the therapist could tailor the mental practice content. Four steps were distinguished: explaining the concept (1), developing imagery techniques (2), applying mental practice (3) and consolidating (4). The protocol had a conditional and an optional part. Patients should have received at least 10 sessions of mental practice (step 2) and have practiced outside of supervised therapy time (24).

In addition, we developed a training program for the therapists, consisting of presentations, workshops, instruction and assisting material and literature.

\section{Does the ability to image automatically imply that neurological patients can and will apply mental} practice?

One major problem remains unresolved. Even if it is possible to teach patients how to image, one cannot know that the patients are actually using the technique: there is no observable behaviour that indicates that the patient is imaging.

There are some techniques that can assist in monitoring and implementing imagery treatments. The SDA-M does allow one to explore what images the patient might be generating, and this was useful to us when developing the treatment program. The ability to perform imagery can also be determined with the hand rotation tests (HRT (25)), muscle activity during imagery with electromyography (EMG (26)) and with eye movements (27). This could be used to investigate a patient's responsiveness to this treatment, but it is not yet proven that the ability to imagine is necessary for the treatment to be effective.

There are research methods for investigating whether a patient is (probably) imaging an action, but these are not applicable in clinical practice. For example, one can compare brain activity during overt movement and imagery with functional magnetic resonance imaging (fMRI (28)), positron emission tomography (PET (29)), blood flow (NIRS) and electroencephalography (EEG (30)). It is important to consider the transfer of positive results from fundamental research in routine care. With the exception of the study by Szameitat and co-workers (31), the skill measured in research studies is often limited to small finger, foot or mouth movements because of the 
constraints of the measuring equipment (32). The terminologies and concepts used by functional neuro-anatomists and imaging scientists to describe movement imagery are not concordant with the concepts of those who focus on the development of skill expertise (32). Third, there is no evidence that reorganizational changes in the brain due to imagery can be associated with increased limb function in larger and less severely impaired stroke samples (33).

Brain imaging tests are not available in routine care, but there are potential clinical methods for investigating what a patient is doing while apparently imaging. Chronometry (comparing the time needed for the execution of the movement with the imagery time) and questionnaires may indicate that a patient is actually imaging. However, recent studies have shown that chronometry posses challenges to assess imagery ability in stroke patients (34). But the Kinesthetic Visual Imagery Questionnaire (35-37) and the hand rotation test (HRT) might help select eligible patients for clinical trials. Up until know, the HRT has been used as part of a testing battery to determine 'chaotic imagery' $(25,38)$ and thereby to select patients for fundamental studies $(39)$. Thus over the last few years, profound contributions to the assessment of imagery abilities in patients have been made. Unfortunately they were not available when we planned our trials, and in any case their clinical utility is still unknown.

Learning and applying mental practice is very complex and maybe our patients were not all able to perform imagery. Patients need a certain cognitive and communication level in order to engage in mental practice (research) (40). Our selection criteria were not 'hard'. Maybe we selected the wrong patient populations?

As discussed above, it is difficult to know to what extent the therapy or movement imagery is actually used. There are no externally observable signs that can confirm that someone understands what mental practice is or is actually undertaken it. We had to rely on self-report (interviews, questionnaires and logs). Little is known about patients' mental practice habits and our studies involved more 'integrity checks' then generally reported in trials (41).

Recent research in sport science suggests that mental imagery may have other, non-motor benefits. This 'second wave of imagery research' has started, to gain more insight on the mental practice habits of athletes $(42,43)$. The research involves young $(44,45)$ and adult athletes, novices and experts (43), healthy and injured (46). Many goals for using mental practice by injured athletes have been identified (46), covering aims within skill acquisition, keeping a high level of motivation and coping with negative thoughts. Maybe we should have added measures of outcome to assess changes in the emotional and cognitive domain. 
At this point it is however unknown if being able to perform imagery necessarily equates to benefiting from it in clinical practice. In addition, our process evaluation showed, that even some people who are able to perform imagery do not want to, and we do not know if people who are unable to perform imagery at baseline, are able to learn it.

\section{Is there less evidence for effects of mental practice than we think there is?}

There has been some criticism of the quality of imagery research in sports $(47,48)$, and these methodological weaknesses may explain why actual effects of mental practice in sports are less than we believed them to be. Two specific matters are of relevance.

First, mental practice has been shown to be effective but effect sizes differ depending on the kind of task that is being researched (49). Mental practice seems to be less effective for improvement of physical performance (strength, endurance, speed) than for improvement of tasks in which coordination or strategy (symbolic learning) plays an important role $(49,50)$.

Second, athletes use mental practice for three domains: improvement of skills, changing emotions and altering cognition (6). These domains need different measures and different approaches. Perhaps effects are a combination of improvement in all three domains. So far, mental practice in neurological rehabilitation has only been used for the improvement of physical skills. More theoretical understanding is needed. In neurological rehabilitation three other reviews $(25,40,51)$ have drawn similar conclusions: mental practice might be effective, but more research is certainly needed.

Most reported imagery research is at the stage of feasibility and piloting: the population sizes are small and relatively few multi-centre studies in clinical routine care $(20,52)$, have taken place. None of the interventions has had sufficient evaluation to come to a conclusion about their effectiveness in a routine clinical setting (52). In addition, the conclusions about effectiveness could be changed by a relatively small number of new trials (52).

Since 2006 only one trial with negative results of mental practice on arm function in chronic stroke out patients was published (53) and there is another unpublished negative trial that we are aware of by Bovend'Eerdt and co-workers (54). Studies with negative outcome are harder to publish than positive ones (publication bias), which might be misleading. 
Should mental practice content be fixed or adjusted to personal preferences, stage of motor learning or skill expertise?

Little is known about how mental practice should be taught and which regimes are most effective. Two aspects are discussed here: (1) the uncertainties of a framework for the therapists and (2) the content of the mental practice intervention.

Developing a framework instead of a fixed protocol has both advantages and disadvantages: a disadvantage was that the flexibility of the framework made the therapists uncertain. Therapists might have preferred a more defined approach. However, mental practice is a complex intervention, which cannot be standardized (1), and so maybe therapists need more training or supervision. On the other hand, during the entire project, therapists were supported and regular visits at the sites took place. Four times I was asked to attend therapy with patients of whom the therapists were uncertain (e.g. if they were practising mentally or therapy content or approach was unclear). Apart from the patient files in which the therapy content was reported, we could not control the way therapists executed the intervention or used imagery, nor how much they stimulated mental practice (55).

In certain stages of motor learning mental practice might cause disbenefit. Masters and coworkers (56) and Beilock and co-workers investigated in sports $(57,58)$ and rehabilitation $(59)$ the phenomenon of paying attention, finding that it might disrupt motor performance of an already automated skill, so called 'choking under pressure' or 'reinvestment'. In patients who tend to reinvest, stepwise imagery as suggested in the framework should only be used in the first phase of motor learning (acquisition). In later stages of motor learning it might be beneficial to divert the attention of the patient away from imaging the movement.

In addition, the stage of motor learning might also affect the relative role of kinesthetic and visual imagery needed to learn the task (32). At present it is known that kinesthetic imagery activates many of the same motor and sensory regions during overt movement whereas visual imagery activates regions primarily concerned with visual processing. Contrary to kinesthetic imagery, visual imagery does not obey Fitts' law $(32,60,61)$. Many researchers therefore promote the use of kinesthetic imagery.

We let the patient decide which view he/she wanted to use: despite differences between visual and kinesthetic imagery, both forms can be used to improve an athlete's performance in a competitive environment. We do not know from clinical research if this is the case for neurological patients. However, (healthy) elderly seem to tend to visual imagery (62) and some patients are simply not able to generate sensory feeling because of their disease. Sometimes there might not be a choice. 
Consequently, the content of mental practice including the chosen point of view should possibly be adjusted or altered depending on the stage of motor learning (expertise). This would make it even more difficult for therapists to tailor mental practice.

\section{Feasibility of and piloting in our studies}

Before establishing the final selection criteria, intervention framework, and additional material and measures, we tested the entire package, including the organization and communication routes. As a final testing step, three case reports were performed. The treatment seemed feasible and first results promising (63). From these preliminary studies we estimated recruitment and made our assumptions for the sample size calculation.

The first trial we prepared was the stroke trial. Its design is reported in chapter five (64). Several aspects of the trial were discussed: the population, the intervention and the measures. We chose the nursing home population of patients suffering from stroke in the (sub) acute phase of recovery for two main reasons. First, patients treated in nursing homes are a big potential target group (65): if mental practice were to be effective, many patients would benefit from implementation. Second, we reasoned that patients in the sub acute phase of recovery were most likely to benefit most from mental practice as plasticity of brain might be used to its full potential during the first three months of recovery $(66,67)$.

The MRC recommends a range of measures of outcome when evaluating a complex intervention, which we had. It seemed reasonable, that if the primary outcome measure (68) would not detect a difference, the measures already used in other studies might, as they had in earlier published relatively small studies.

Results from our pilot work showed no reason to adjust the mental practice intervention itself but we identified the need for development of better training of therapists and organization of treatment delivery. It was also important to repeat the piloting work at every involved research location because the organization is always specific for the setting.

Even though we performed several pilot studies in stroke, testing also organization routes, we underestimated the difficulties in recruitment that became apparent when we performed the multi-centre trial. Complex clinical pathways in stroke rehabilitation probably influenced recruitment negatively as well: for example we could not recruit patients admitted to the nursing home if it was planned that they would move on to a specialist rehabilitation centre. In the Parkinson's study we had a much more stable situation. But we perhaps overestimated the effect size, leading to too small sample sizes. 


\section{Does preliminary work lead to the preparation of trials?}

Many feasibility studies been performed in mental practice (e.g. $(69,70)$, but is not clear to which extend the results have been used for input of trials with full evaluation. Apart from testing the feasibility of a complex intervention, it is important to understand the context in which interventions take place. The population characteristics, given cultural norms, teaching styles, organization of clinical pathways, relationships between therapists and patients should also be taken into account when determining the feasibility of mental practice (1). All these variables may influence the assumptions about the sample size calculation (effects smaller or response rates lower).

\section{Evaluation of both trials}

Full evaluation of complex interventions consists of three assessments: effectiveness, cost effectiveness and understanding change process (1). In both the stroke trial and Parkinson's trial we performed an effectiveness and process evaluation.

Despite the suggested working mechanism, promising results of earlier research and our profound preliminary work on optimizing the treatment, training the therapists and selecting the patient, none of the measures revealed any effect in favour of the experimental group at any of the measuring points (chapter seven (71)).

Results from the Parkinson's trial are reported in chapter eight (72). Mental practice seems to be more feasible for patients with minor to mild stages of the disease (Hoehn and Yahr stage $<3$ ). The sub group analyses showed effects in favour of the experimental intervention, but the groups were too small for differences to be statistically significant.

We performed a process evaluation of both trials in order to get more insight into elements that might have influenced the results. The process evaluation of the stroke trial is reported in chapter six (24). We were confronted with two main challenges. The majority of participants did not receive the entire framework in six weeks time although most patients received the minimum amount of mental practice. Some therapists were uncomfortable with the flexibility of the framework. This left us wondering if (a) patients were able to apply mental practice (b) patients had practiced enough to make a (measurable) difference and (c) therapists had facilitated imagery use sufficiently. On the other hand, results from our qualitative research project in which we assessed mental practice habits from patients suffering from stroke and of patients with Parkinson's disease, were mainly positive (chapter nine (41)). 
All interviewed stroke patients perceived benefits from the use of mental practice, such as more influence on the rehabilitation process, improved performance of skills, less anxiety during physical activity, increased secure feeling and motivation for therapy. Although patients with Parkinson's disease reported more problems with and effort in applying mental practice, they reported that they attained more control over their motor performance. Apparently, most patients had practiced and perceived benefits, but it was not detected at the physical performance level.

\section{Have full evaluations (effect, cost and process) of mental practice interventions within clinical trials in} neurological conditions taken place?

We are not aware of any process evaluation of mental practice interventions in neurological populations. Recent reviews on the evidence for specific physical therapy interventions in stroke and Parkinson's disease have concluded that there is a real need to focus on a smaller number of robust, standardized, and relevant outcome measures and to ensure that all trials include these measures in a common dataset to enable future comparison $(20,52,73,74)$. Not only the outcome should be reported, but also the process evaluation and clear description of the intervention to enable replication of research and increase power (synthesis of evidence).

\section{Where does ongoing research in other pathologies stand?}

More research groups are assessing the use of mental practice in more different patient groups. The last decade mental practice has been used in patients with cancer, chronic pain and spinal cord injury $(51,75)$ and as part as treatment regimes involving mirror therapy in patients after amputation and chronic limb pain (76). Recently, initial evidence for the use of mental practice in flexor tendon repair has been presented (77).

Up until now, mental practice has only been used in neurological populations to improve performance on a physical level (impairment (e.g. spasticity $(78)$ ) or activity (e.g. gait $(15,70)$ ). In other populations it has however also been used for other aims, such as improving quality of life (cancer research) or reducing pain. Simmons and co-workers are one of the few researchers that have published results of what patients do during mental practice (38). Qualitative research could be another way of understanding mental processes in patients better.

Process evaluations in chronic limb pain and in cancer research have been reported (75). In a study by Moseley and co-workers the use of mental practice in some chronic pain patients lead to an increase in perceived pain when imagining movements (76). As far as we know, no negative side effects have been investigated in neurological rehabilitation: reinvestment of effort so that automated skills are in fact impaired, may be one such negative effect. 


\section{Implementation recommendations}

We do not (yet) recommend implementation of the described mental framework on a large scale in the therapy of either patients suffering from stroke in the sub acute phase of recovery or in patients with Parkinson's disease.

In general it is hard to recommend specific approaches or interventions for implementation in rehabilitation of neurological populations: clinical trial evidence is based on very selected populations (52), e.g. people with communication problems are often excluded from research (79). Research in 'fashionable' interventions should take place early on so sufficient evidence reveals whether a therapy form is effective or not and in which patients it should be applied. Possibly as research methodology improves and initial enthusiasm wanes, a more realistic evaluation arises. It is therefore advised to further use mental practice in research projects. Randomisation should always be considered, but other designs may be more appropriate, depending on expected effect size and likelihood of selection or allocation bias (1). Long term follow-up is needed to assess whether a mental practice intervention has a cumulative effect.

\section{Where should we go from here?}

A Dutch psychologist once wrote: 'We call something 'normal' because it happens a lot, not because we understand it' (80). We believe the same accounts for movement imagery and mental practice and that it would be useful to study the phenomenon further. It may help.

The focus of future research should be on:

\section{Development:}

- study underlying mechanisms of why mental practice works in some patients and does not in others (individually and disease related);

- predict who is able to perform and benefit from mental practice (selection and prognosis);

- assess whether mental practice interventions need to be adjusted to stage of motor recovery, complexity of the skill and specific patient populations.

\section{Feasibility and piloting:}

- determining and evaluating clinical pathways to gain a more realistic view on recruitment;

- determine possible negative side effects of mental practice.

\section{Evaluating:}

- select the best measuring instruments to determine effects on different levels (cognition, emotion and motor performance);

- and make results from trials comparable;

- assess mental practice behaviour through qualitative research;

- evaluate processes and cost effectiveness. 
Results from future studies will determine whether mental practice should be implemented in routine care and if so, what the best way is.

\section{And finally}

Who could have imagined five years ago what kind of effect a PhD-research project would have on education and care in the South of the Netherlands and beyond (fig. 3)?

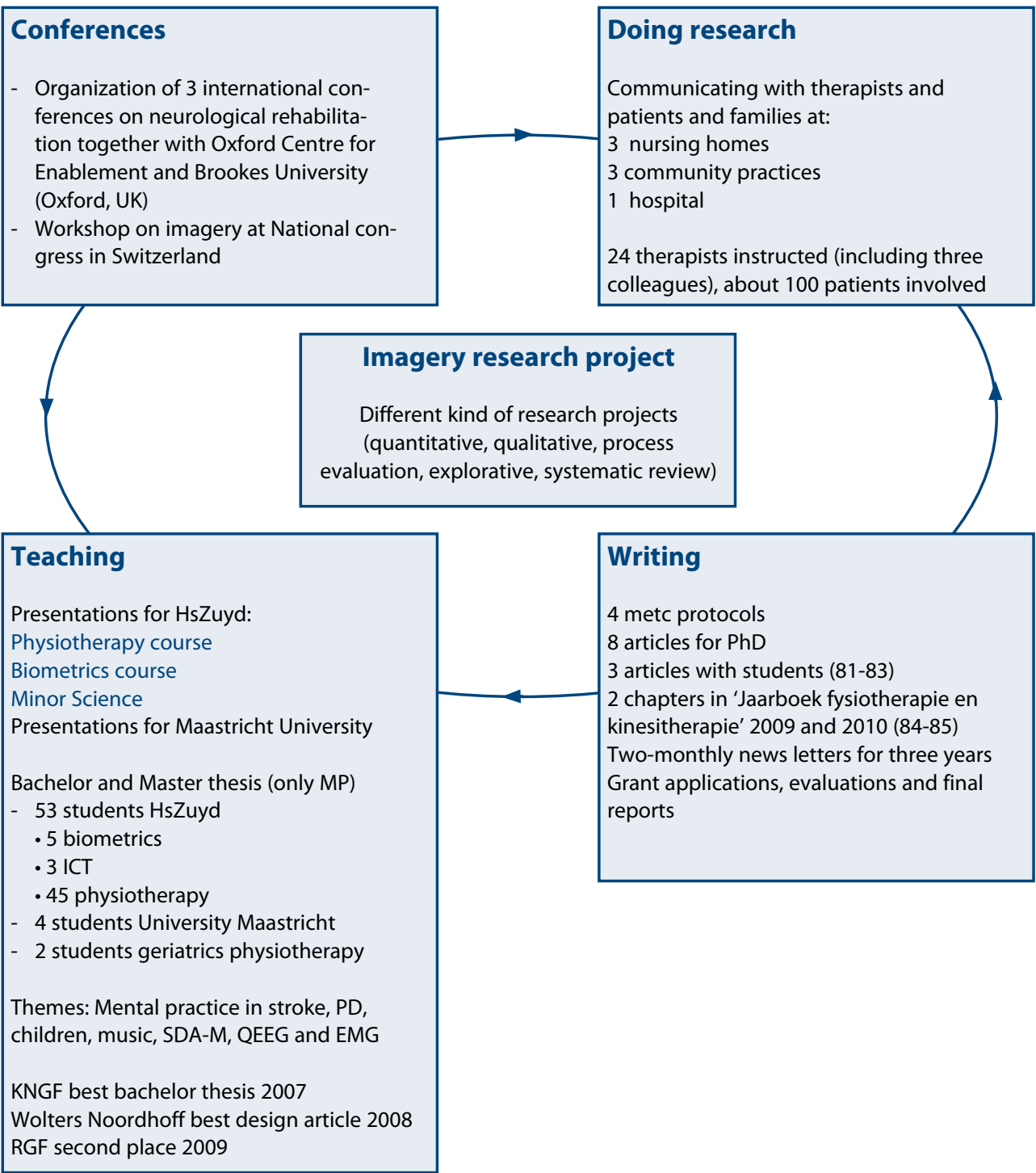

Fig. 3: Overview of the most prominent activities that were part of or generated by the research project. 


\section{References}

1. Craig P, Dieppe P, Macintyre S, Michie S, Nazareth I, Petticrew M. Developing and evaluating complex interventions: the new Medical Research Council guidance. Bmj 2008;337:a1655.

2. Mellit E, Petit L. Reopening the mental imagery debate: Lessons from functional anatomy. Neuroimage 1998;8:129-39.

3. Decety J, Perani D, Jeannerod M, Bettinardi V, Tadary B, Woods R, et al. Mapping motor representations with positron emission tomography. Nature 1994;371:600-2.

4. Jackson PL, Lafleur MF, Malouin F, Richards C, Doyon J. Potential role of mental practice using motor imagery in neurologic rehabilitation. Arch Phys Med Rehabil 2001;82:1133-41.

5. van Leeuwen R, Inglis J. Mental practice and imagery: a potential role in stroke rehabilitation. Physical Therapy Reviews 1998:47-52.

6. Martin K, Moritz S, Hall C. Imagery use in sports: a literature review and applied model. The Sports Psychologist 1999;13:245-68.

7. Gould D, Damarjian N, Greenleaf C. Imagery training for peak performance. In: Raalte van J, Brewer WB, editors. Exploring sport and exercise psychology. Washington (DC): Am Psychol Assoc; 2002. p 49-74.

8. Isaac A, Marks, D, Russel, D. An Instrument for Assesing Imagery of Movement: The Vividness of Movement Imagery Questionaire (VMIQ). Journal of Mental Imagery 1986;10:23-30.

9. Schack $\mathrm{T}$. The relationship between motor representation and biomechanical parameters in complex movements. Towards an integrative perspective of movement science. Eur J Sport Sci 2003; 3:1-13.

10. Braun SM, Schack T, Marcellis RG, Oti KC, Schols JMGA, Wade DT, Beurskens AJ. Is it possible to use Structural Dimension Analysis of Motor memory (SDA-M) to investigate representations of motor actions in stroke patients? Clin Rehabil 2007;21:822-32.

11. Tombaugh TN, McIntyre NJ. The mini-mental state examination: a comprehensive review. J Am Geriatr Soc 1992;40:922-35.

12. Braun SM, Beurskens AJ, Borm PJ, Schack T, Wade DT. The effects of mental practice in stroke rehabilitation: a systematic review. Arch Phys Med Rehabil 2006;87:842-52.

13. Yaguez L, Canavan AG, Lange HW, Homberg V. Motor learning by imagery is differentially affected in Parkinson's and Huntington's diseases. Behav Brain Res 1999;102:115-27.

14. Frak V, Cohen H, Pourcher E. A dissociation between real and simulated movements in Parkinson's disease. Neuroreport 2004;15:1489-92.

15. Tamir R, Dickstein R, Huberman M. Integration of motor imagery and physical practice in group treatment applied to subjects with Parkinson's disease. Neurorehabil Neural Repair 2007;21:68-75. 
16. Shumway-Cook A, Woollacott MH. Motor control: translating research into clinical practice. 3rd edition ed. Philadelphia: Lippincott Williams \& Wilkins; 2006.

17. van Peppen RP KG, Hermeling-van der Wel, BC et al. KNGF-richtlijn beroerte. Ned Tijdschr Fysiother 2004;114(Suppl):1-77.

18. Keus SHJ, Hendriks HJM, Bloem BR, Bredero-Cohen AB, de Goede CJT, van Haaren M, et al. KNGF-Richtlijn ‘Parkinson'. Ned Tijdschr Fysiother 2004;114(Suppl):1-86.

19. Commissie CVA-revalidatie. Revalidatie na een beroerte, richtlijnen en aanbevelingen voor zorgverleners. Den Haag: Nederlandse Hartstichting; 2001.

20. Kwakkel G, de Goede CJ, van Wegen EE. Impact of physical therapy for Parkinson's disease: a critical review of the literature. Parkinsonism Relat Disord 2007;13:S478-87.

21. Kwakkel G, van Peppen R, Wagenaar RC, Wood Dauphinee S, Richards C, Ashburn A, et al. Effects of augmented exercise therapy time after stroke: a meta-analysis. Stroke 2004;35: 2529-39.

22. Nijhuis-van der Sanden R. Quid movet? Inauguration; 2009 September 24th; Nijmegen, the Netherlands. Drukkerij Roos en Roos Arnhem; 2009. p 5-28.

23. Braun SM, Kleynen M, Schols JMGH, Schack T, Beurskens AJ, Wade DT. Using mental practice in stroke rehabilitation; a framework. Clin Rehabil 2008;22:579-591.

24. Braun SM, van Haastregt JC, Beurskens AJ, Wade DT, Gielen X, Schols JM. Feasibility of a mental practice intervention in stroke patient in nursing homes; a process evaluation. (submitted 2010).

25. Sharma N, Pomeroy VM, Baron JC. Motor imagery: a backdoor to the motor system after stroke? Stroke 2006;37:1941-52.

26. Bakker FC, Boschker MSJ, Chung T. Changes in muscular activity while imagining weight lifting using stimulus or response proportions. Journal of Sport \& Exercise Psychology 1996;18:313-24.

27. Heremans E, Helsen WF, De Poel HJ, Alaerts K, Meyns P, Feys P. Facilitation of motor imagery through movement-related cueing. Brain Res 2009;1278:50-8.

28. Johnson SH, Sprehn G, Saykin AJ. Intact motor imagery in chronic upper limb hemiplegics: evidence for activity-independent action representations. J Cogn Neurosci 2002;14:841-52.

29. Malouin F, Richards CL, Jackson PL, Dumas F, Doyon J. Brain activations during motor imagery of locomotor-related tasks: a PET study. Hum Brain Mapp 2003;19:47-62.

30. Pfurtscheller G, Brunner C, Schlogl A, Lopes da Silva FH. Mu rhythm (de)synchronization and EEG single-trial classification of different motor imagery tasks. Neuroimage 2006;31: 153-9.

31. Szameitat AJ, Shen S, Sterr A. Motor imagery of complex everyday movements. An fMRI study. Neuroimage 2007;34:702-13. 
32. Milton J, Small SL, Solodkin A. Imaging motor imagery: methodological issues related to expertise. Methods 2008;45:336-41.

33. Johnson-Frey SH. Stimulation through simulation? Motor imagery and functional reorganization in hemiplegic stroke patients. Brain Cogn 2004;55:328-31.

34. Malouin F, Richards CL, Durand A, Doyon J. Reliability of mental chronometry for assessing motor imagery ability after stroke. Arch Phys Med Rehabil 2008;89:311-9.

35. Malouin F, Richards CL, Durand A, Descent M, Poire D, Fremont P, et al. Effects of Practice, Visual Loss, Limb Amputation, and Disuse on Motor Imagery Vividness. Neurorehabil Neural Repair 2009;23:449-63.

36. Malouin F, Richards CL, Durand A, Doyon J. Clinical assessment of motor imagery after stroke. Neurorehabil Neural Repair 2008;22:330-40.

37. Malouin F, Richards CL, Jackson PL, Lafleur MF, Durand A, Doyon J. The Kinesthetic and Visual Imagery Questionnaire (KVIQ) for assessing motor imagery in persons with physical disabilities: a reliability and construct validity study. J Neurol Phys Ther 2007;31:20-9.

38. Simmons L, Sharma N, Baron JC, Pomeroy VM. Motor imagery to enhance recovery after subcortical stroke: who might benefit, daily dose, and potential effects. Neurorehabil Neural Repair 2008;22:458-67.

39. Sharma N, Simmons LH, Jones PS, Day DJ, Carpenter TA, Pomeroy VM, et al. Motor imagery after subcortical stroke: a functional magnetic resonance imaging study. Stroke 2009;40:1315-24.

40. Malouin F, Richards CL. Mental Practice for Relearning Locomotor Skills. Phys Ther 2010;90:240-51.

41. Braun SM, Beurskens AJ, Dalemans R, Schols JM, Wade DT. The experience of movement imagery therapy by patients with stroke or Parkinson's disease. (Submitted 2009)

42. Munroe K, Giacobbi P, Hall C, Weinberg R. The four w's of imagery use: Where, when, why and what. The Sport Psychologist 2000;14:119-37.

43. MacIntyre TE, Moran AP. A qualitative investigation of imagery use and meta-imagery processes among elite canoe-slalom competitors. Journal of imagery research in sport and physical activity 2007;2:1-19.

44. Munroe-Chandler K, Hall C, Fishburne G. Playing with confidence: The relationship between imagery use and self-confidence and self-efficacy in youth soccer players. J Sports Sci 2008;23:1-8.

45. Munroe-Chandler KJ, Hall CR, Fishburne GJ, Strachan L. Where, when, and why young athletes use imagery: an examination of developmental differences. Res $Q$ Exerc Sport 2007;78:103-16.

46. Driediger M, Hall C, Callow N. Imagery use by injured athletes: a qualitative analysis. J Sports Sci. 2006;24:261-71. 
47. Driskell JE, Copper C, Moran A. Does mental practice enhance performance? Journal of applied psychology 1994;79:481-92.

48. Budney AJ, Murphy SM, Woolfolk RL. Imagery and Motor performance: What do we really know? In: Sheikh AA, Korn ER, editors. Imagery in Sports and Physical Performance. Farmingdale, NY: Baywood; 1994. p. 97-120.

49. Feltz D, Landers D. A revised meta-analysis of the mental practise literature on motor skill learning and performance. In: Druckman D. SJ, editor. Enhancing human performance: Issues, theories and techniques. Washington DC: National Academy of Science; 1988. p. 1-65.

50. Sackett RS. The relationship between amout of symbolic rehearsal and retention of a maze habit. Journal of General Psychology 1935;13:113-28.

51. Dickstein R, Deutsch JE. Motor imagery in physical therapist practice. Phys Ther 2007;87: 942-53.

52. Langhorne P, Coupar F, Pollock A. Motor recovery after stroke: a systematic review. Lancet Neurol 2009;8:741-54.

53. Dijkerman HC, Letswaart M, Johnston M, MacWalter RS. Does motor imagery training improve hand function in chronic stroke patients? A pilot study. Clin Rehabil 2004;18:538-49.

54. Bovend'Eerdt T. Preliminary results of a RCT using an integrated motor imagery programme. Third Heerlen - Oxford conference on imagery and neurological rehabilitation. 2009, May 14th; Heerlen. p 5.

55. Jedlic B, Hall N, Munroe-Chandler K, Hall C. Coaches' encouragement of athletes' imagery use. Res Q Exerc Sport 2007;78:351-63.

56. Masters RSW, Maxell JP. The theory of reinvestment. International Review of Sports and Exercise Psychology 2008;1:160-83.

57. Beilock SL, Gonso S. Putting in the mind versus putting on the green: expertise, performance time, and the linking of imagery and action. Q J Exp Psychol 2008;61:920-32.

58. Beilock SL, Carr TH, MacMahon C, Starkes JL. When paying attention becomes counterproductive: impact of divided versus skill-focused attention on novice and experienced performance of sensorimotor skills. J Exp Psychol Appl 2002;8:6-16.

59. Orrell AJ, Masters RS, Eves FF. Reinvestment and movement disruption following stroke. Neurorehabil Neural Repair 2009;23:177-83.

60. Grosjean M, Shiffrar M, Knoblich G. Fitts's law holds for action perception. Psychol Sci 2007;18:95-9.

61. Fitts PM, Posner MI. Human performance. Belmont, CA: Books / Cole Publishing Company; 1967.

62. Mulder T, Hochstenbach JB, van Heuvelen MJ, den Otter AR. Motor imagery: the relation between age and imagery capacity. Hum Mov Sci 2007;26:203-11. 
63. Kleynen M, Schoenmakers M, Braun SM. Stel je voor, het werkt. Mentale training bij een chronische CVA patiënt: een case report. Ned Tijdschr Fysiother 2009;119:48-54.

64. Braun SM, Beurskens AJ, van Kroonenburgh SM, Demarteau J, Schols JM, Wade DT. Effects of mental practice embedded in daily therapy compared to therapy as usual in adult stroke patients in Dutch nursing homes: design of a randomised controlled trial. BMC Neurol 2007;7:34.

65. Schols JM, Crebolder HF, van Weel C. Nursing home and nursing home physician: the Dutch experience. J Am Med Dir Assoc 2004;5:207-12.

66. Kleim JA, Jones TA. Principles of experience-dependent neural plasticity: implications for rehabilitation after brain damage. J Speech Lang Hear Res 2008;51:225-39.

67. Burke SN, Barnes CA. Neural plasticity in the ageing brain. Nat Rev Neurosci 2006;7:30-40.

68. Wewers ME, Lowe NK. A critical review of visual analogue scales in the measurement of clinical phenomena. Res Nurs Health 1990;13:227-36.

69. Malouin F, Richards CL, Doyon J, Desrosiers J, Belleville S. Training mobility tasks after stroke with combined mental and physical practice: a feasibility study. Neurorehabil Neural Repair 2004;18:66-75.

70. Dunsky A, Dickstein R, Ariav C, Deutsch J, Marcovitz E. Motor imagery practice in gait rehabilitation of chronic post-stroke hemiparesis: four case studies. Int J Rehabil Res 2006;29:351-6.

71. Braun SM, Beurskens AJ, Kleynen M, Oudelaar B, Schols JM, Wade DT. Effects of mental practice in stroke rehabilitation in Dutch nursing homes: results from a randomised controlled trial. (submitted 2010)

72. Braun SM, Beurskens AJ, Kleynen M, Schols JM, Wade DT. Using mental practice in patients with Parkinson's disease; results from a randomised controlled trial. (submitted 2010)

73. van Peppen RP, Hendriks HJ, van Meeteren NL, Helders PJ, Kwakkel G. The development of a clinical practice stroke guideline for physiotherapists in the Netherlands: a systematic review of available evidence. Disabil Rehabil 2007;29:767-83.

74. Dibble LE, Addison O, Papa E. The effects of exercise on balance in persons with Parkinson's disease: a systematic review across the disability spectrum. J Neurol Phys Ther 2009;33:14-26.

75. Freeman L, Cohen L, Stewart M, White R, Link J, Palmer JL, et al. The experience of imagery as a post-treatment intervention in patients with breast cancer: program, process, and patient recommendations. Oncol Nurs Forum 2008;35:116-21.

76. Moseley GL. Graded motor imagery is effective for long-standing complex regional pain syndrome: a randomised controlled trial. Pain 2004;108:192-8.

77. Stenekes MW, Geertzen JH, Nicolai JP, De Jong BM, Mulder T. Effects of motor imagery on hand function during immobilization after flexor tendon repair. Arch Phys Med Rehabil 2009;90:553-9. 
78. Bovend'Eerdt TJ, Dawes H, Sackley C, Izadi H, Wade DT. Mental techniques during manual stretching in spasticity - a pilot randomized controlled trial. Clin Rehabil 2009;23:137-45.

79. Dalemans R, Wade DT, van den Heuvel WJ, de Witte LP. Facilitating the participation of people with aphasia in research: a description of strategies. Clin Rehabil 2009;23:948-59.

80. Vroon P. Psychologie in het dagelijks leven. Amsterdam: Ambo; 1983.

81. van Elteren D, Vonk JV, van Engelen E, Braun SM. De "Structural Dimension Analysis of Motor memory" (SDA-M) in een centraal neurologische revalidatie setting. Stimulus 2009;2:180-197

82. Schmitz E, Friedrich S, Beurskens AJ, Wade DT, Braun SM. The effects of mental practice in children: a systematic review. (submitted 2010)

83. Mey M, Herzing K, Braun SM, Rothgangel A. Die Wii Konsole und Patienten mit Morbus Parkinson: eine Patientenserie. (accepted 2010)

84. Braun SM. Het gebruik van bewegingsvoorstellingen in de revalidatie: Hoe maak je de transfer van sport naar therapie? In: Jaarboek Fysiotherapie/Kinesitherapie 2009. Geraets J.J.X.R. et al (eds). Houten: Bohn Stafleu van Loghum; 2009. p 191-201

85. Braun SM. Mentale training in de neurologische revalidatie: veranderen van motoriek, cognities en emoties. In: Jaarboek Fysiotherapie/Kinesitherapie 2010. Veenhof C. et al (eds). Houten: Bohn Stafleu van Loghum; 2010. p 244-256. 


\section{Summary}

While it is reasonably established that the overall process of neurological rehabilitation is effective, there is little evidence to support many specific rehabilitation therapeutic techniques. Currently it seems that task orientated practice (i.e. practising an activity of relevance) is the most effective single therapeutic technique. This is similar to the situation in sport where practice is the bedrock of improving skills. Indeed improving any skilled motor activity seems to depend upon continuing practice. Mental practice may be another way of practising. Starting from research in sports science, we developed and evaluated a mental practice intervention in neurorehabilitation.

In chapter one the topic is introduced, starting with the evidence and use of mental practice in sports.

Before we started our clinical studies, we wanted to know how much evidence there was for mental practice in stroke rehabilitation. In chapter two, our systematic review is described. Ten articles on mental practice in stroke rehabilitation were included in our review. In nine studies mental practice lead to a significant improvement. The mental practice treatments were less complex (e.g. tape recording) than the framework we developed in eight of the studies. No training of therapists was reported. No sub groups within the stroke population seemed to benefit more from mental practice and no treatment content or regimes seemed to work better.

In sports science, a computer program to assess the cognitive structure of motor skills, the Structural Dimension Analysis of Motor memory (SDA-M), was developed to distinguish differences in motor representations between novices and experts. Results in chapter three show that it also seemed feasible in stroke patients but assessment was time consuming and little is known about its validity (which is based on face validity and expert opinion). The interpretation of the tree diagrams was usable in clinical practice when analyzing a single patient's performance, but the results from the SDA-M where hard to quantify as an outcome measure.

The development of a mental practice intervention was also an attempt to define mental practice and unify the mental practice intervention and describe how mental practice could be taught in rehabilitation. In chapter four the framework we developed is described, in which the therapist could tailor the mental practice content. Four steps were distinguished: (1) explaining the concept, (2) developing imagery techniques, (3) applying mental practice and (4) consolidating. 
The protocol had a mandatory and an optional part. Patients should have received at least 10 sessions of mental practice (step 2) and have practiced outside of supervised therapy time (24).

Because the effects of mental practice in stroke rehabilitation were still uncertain, we decided to do a clinical study on effects of imagery in stroke patients, in the sub acute phase of recovery, in nursing homes. The underlying thoughts and decisions were reported in a design article in chapter five. We specifically chose this sub group within the stroke population for three reasons: (1) They are a large part of the entire population and if imagery were effective in these patients a large group would benefit from it; (2) we reasoned that patients in the sub acute phase of recovery were most likely to benefit most from mental practice as change is greatest over the first three months of main recovery; and (3) we hypothesized that patients might recall the sensations of normal motor control and movement better shortly after their stroke than months later. The Medical Research Council recommends a range of measures of outcome when evaluating a complex intervention, which we had. It seemed reasonable that if the primary outcome measure did not detect a difference, the measures already used in other studies might, just as they had in earlier published relatively small studies.

In chapter six the treatment process evaluated in the randomised controlled trial was studied in a detailed process evaluation. The aims of this study were to assess (1) whether the mandatory and optional part of the mental practice intervention were performed according to the framework and (2) the therapists' and participants' experiences of the intervention. We were confronted with two main challenges. The majority of participants did not receive the entire framework in six weeks time although most patients received the minimum amount of mental practice. Some therapists were uncomfortable with the flexibility of the framework.

The results from a randomised controlled trial in 36 stroke patients are reported in chapter seven. Despite the theoretical support, the promising results of earlier research and our preliminary work on optimizing the treatment, training the therapists and selecting the patients, none of the measures revealed any effect in favour of the experimental group at any of the measuring points.

Another randomized controlled trial with patients with Parkinson's disease in the community was also carried out and results are reported in chapter eight. Mental practice seemed to be more feasible for patients with minor to mild stages of the disease (Hoehn and Yahr stage $<3$ ). The sub group analyses showed effects in favour of the experimental intervention in less severely affected patients, but the groups were too small for differences to be statistically significant. 
In chapter nine we report on interviews with patients after stroke or with Parkinson's disease who had received mental practice training. All interviewed stroke patients perceived benefits from the use of mental practice, such as more influence on the rehabilitation process, improved performance of skills, less anxiety during physical activity, increased secure feeling and motivation for therapy. Although patients with Parkinson's disease reported more problems with and effort in applying mental practice, they reported that they attained more control over their motor performance. Apparently, most patients had practiced and perceived benefits, but it was not (always) detected at the physical performance level.

In chapter ten we discuss more generally the results of this $\mathrm{PhD}$-project. The guidance from the Medical Research Council for developing and evaluating complex interventions is used to compare our findings with results from other research. Where do we stand at this point? And where should future imagery research focus on?

We do not (yet) recommend implementation of the described mental framework on a large scale in the therapy of either patients suffering from stroke in the sub acute phase of recovery or in patients with Parkinson's disease. Results from future studies will determine whether mental practice should be implemented in routine care and if so, what the best way is. 


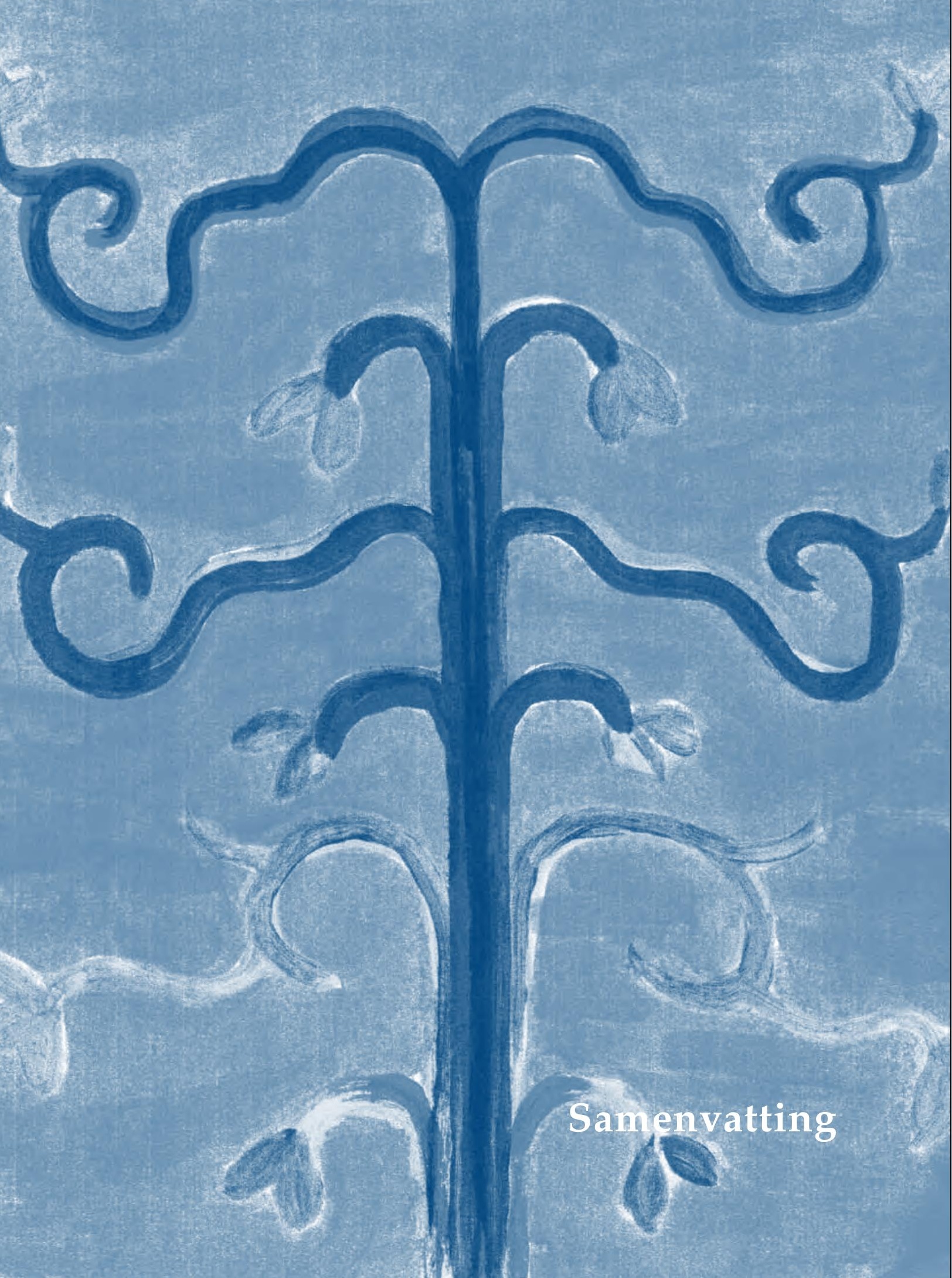




\section{Samenvatting (Dutch summary)}

Het voorliggende proefschrift heeft als thema 'het gebruik van mentale training binnen de neurologische revalidatie'. Onderstaande achtergrondinformatie vormde de basis voor het onderzoeksproject:

'Alle bewegingen worden eerst bewust aangeleerd en cognitief aangestuurd c.q. gecontroleerd. Met de tijd, door het constant oefenen, worden ze geleerd en geautomatiseerd. Leren impliceert dat er informatie wordt verwerkt en dat het geleerde sporen achterlaat in de hersenen door neurale routes te verstevigen of nieuwe neurale verbindingen te maken. Deze neurale prints kunnen geactiveerd worden als een specifieke bewegingsafloop gevraagd wordt. Ze worden waarschijnlijk opgeslagen als een specifiek patroon van interacties (set). Elke set is een motor plan dat overeenkomt met een min of meer specifiek patroon van spieractiviteit en de set stuurt deze spieractiviteit ook aan. Als er beschadiging van de hersenen plaatsvindt door een ziekte, dan kan het gevolg zijn dat ook bepaalde neurale routes en verbindingen verbroken worden, wat wederom kan leiden tot een ontwrichting van het motor plan of in het ergste geval, verlies hiervan. Bij het nastreven van herstel of verbetering van dit verlies van neurologisch weefsel, zullen oude neurale routes hersteld of nieuwe ontwikkeld moeten worden. In dit promotieonderzoek is het potentieel van mentale training als additionele therapievorm voor mensen na een beroerte en met de ziekte van Parkinson om nieuwe motorische vaardigheden te leren, onderzocht'.

\section{Aanleiding}

Atleten gebruiken veel verschillende psychologische vaardigheden om tot topprestaties te komen, zoals focussen, zichzelf oppeppen, motiveren door het stellen van doelen en mentale training. De meeste mensen kennen het gebruik van bewegingsvoorstellingen en mentale training vanuit de sport. Aan de gesloten ogen en lichaamsbewegingen ziet men op t.v. skiërs die voor hun race de afdaling in hun hoofd (mentaal) doornemen, hoogspringers die hun sprong visualiseren en zwemmers of schaatsers die hun wedstrijd in gedachten perfectioneren. De mentale training is er dan op gericht om bewegingen te leren, bewegingsaflopen te veranderen, te stabiliseren of te optimaliseren. Movement imagery wordt beschreven als 'quasi-perceptuele ervaringen van bewegingen, waarvan men zich bewust is en die zich voordoen zonder dat de motorische prikkels aanwezig zijn die deze ervaringen normaal gesproken oproepen' (Nederlands: bewegingsvoorstelling). Mental practice verwijst naar 'een bepaalde techniek die door atleten gebruikt wordt om specifieke bewegingen of motorische vaardigheden te leren, oefenen of perfectioneren zonder daadwerkelijk te bewegen' (Nederlands: mentale training). Tijdens de bewegingsvoorstelling wordt een interne neurale representatie van de beweging geactiveerd en de bewegingsafloop herhaaldelijk in gedachten in een specifieke situatie geoefend (context gebonden), zonder daadwerkelijk fysiek te bewegen. 
Naast het gebruik van mentale training tijdens wedstrijden en trainingen wordt mentale training door sporters ook gebruikt gedurende een blessureperiode. Het gebruik van imagery door revalidanten kan nog het beste vergeleken worden met dat van geblesseerde sporters. Imagerygedrag van een kleine groep geblesseerde atleten is al eens in kaart gebracht. Naast het verbeteren van de motoriek gebruiken atleten imagery bijvoorbeeld ook om angst te verminderen en om beter om te gaan met pijn. Atleten blijken individuele voorkeuren te hebben voor het gebruik van mentale training. Oefentijden varieerden van vijf tot 30 seconden. Sommige atleten konden allerlei sensaties oproepen (zoals geur, lichaamsgevoel, gehoor), anderen gebruikten één bepaalde sensatie. De meeste atleten geloofden baat te hebben bij het gebruik van imagery. Mentale training heeft de afgelopen eeuw behoorlijk wat critici gekend. Een reden daarvoor was dat lange tijd geen enkele theorie in zijn geheel de effecten van mentale training kon verklaren. Hierin kwam verandering met de komst van hersenactivatieonderzoek in de jaren 80 en 90 van de vorige eeuw. De technologie achter functional magnetic resonance imaging (fMRI) en positron emission tomography (PETscans) maakte een grote ontwikkeling door. Uit studies met fMRI en PET bleek namelijk dat tijdens het voorstellen van een beweging vrijwel dezelfde hersengebieden actief zijn als tijdens het daadwerkelijk uitvoeren van deze beweging.

\section{Complexe interventie}

Mentale training is een complexe interventies. Indien complexe interventies toegepast gaan worden bij een nieuwe doelgroep, zullen deze waarschijnlijk bijgesteld, ontwikkeld en geëvalueerd moeten worden. Hierbij worden vier stappen onderscheiden: (1) achtergrond theorie verduidelijken, modelleren; (2) eerste toetsing interventie met behulp van hanteerbaarheids- en pilotstudies; (3) doorvoeren van grotere studies waarbij gerandomiseerd wordt met uitvoerige evaluaties en (4) mogelijk implementatie. Binnen het voorliggende onderzoeksproject hebben een aantal deelonderzoeken plaatsgevonden (stap 1, 2 en 3). Dit heeft ertoe geleid dat het onderzoeksproject gefaseerd heeft plaatsgevonden. In het eerste deel van het traject hebben we ons vooral bezig gehouden met de theoretische achtergrond van het onderwerp: welke bewijskracht is er voor mentale training (hoofdstuk 2) en hoe zouden bewegingenvoorstellingen gemeten kunnen worden (hoofdstuk 3)? Deze stappen waren van belang om tot de inhoud van een mentale training interventie te komen (hoofdstuk 4) en onze studies te kunnen plannen (hoofdstuk 5). Figuur 1 geeft hiervan een overzicht. 


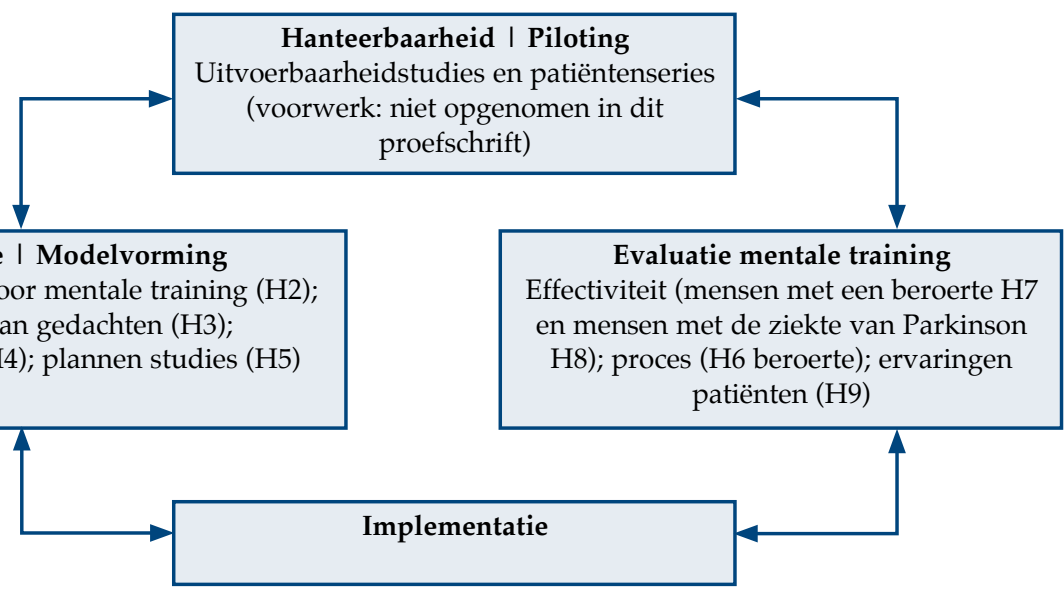

Fig. 1: Vier stappen binnen het ontwikkelen en evalueren van complexe interventies volgens het model van de medische onderzoeksraad (Medical Research Counsil; MRC, Craig et al. 2008).

In het tweede deel van het traject hebben we onze ideeën getoetst in de praktijk bij twee doelgroepen: patiënten na een beroerte en patiënten met de ziekte van Parkinson. De effecten van beide afzonderlijke studies zijn beschreven (hoofdstukken 7 en 8) en er wordt verslag gedaan van de ervaringen van patiënten uit deze twee doelgroepen met mentale training (hoofdstuk 9). Van de studie met patiënten na een beroerte is tevens een proces evaluatie beschreven (hoofdstuk 6).

\section{Deel 1: Voorbereidend werk}

Bewijs voor effecten van mentale training in de revalidatie na een beroerte en de ziekte van

\section{Parkinson}

Er is onderzoek gedaan onder gezonde jongeren, maar er is nog erg weinig fundamenteel onderzoek met fMRI en PET gedaan bij oudere proefpersonen en bij patiënten met centraal neurologische aandoeningen. In hoeverre de lokalisatie van de laesie invloed heeft op hersenactivatiepatronen of op de kwaliteit van imagery is nog onduidelijk. Onduidelijk is ook welke invloed een veranderde hersenactivatie heeft op mogelijke effecten van mentale training. Het is van belang hier meer onderzoek naar te doen.

Veel patiënten klagen na een beroerte over vermoeidheid en een verminderde conditie. Dit is een grote fysieke belemmering in het trainen van deze patiënten. Imagery lijkt een goed alternatief om veilig de therapieomvang te vergroten. De eerste therapeutische toepassingen van mentale training bij patiënten met een beroerte (CVA; cerebrovasculair accident) werden vanaf 2000 
beschreven. In onze eigen review uit 2006 concludeerden we dat mentale training als additionele therapie mogelijk zou kunnen bijdragen tot een verbeterd herstel. Echter, de geïncludeerde studies waren moeilijk met elkaar te vergelijken: de mentale trainingsinhoud varieerde en de gebruikte meetinstrumenten en uitkomstmaten verschilden. Hoewel sluitende conclusies niet getrokken konden worden, leek er toch 'muziek' te zitten in de behandeling van CVA-patiënten met mentale training. Andere, later gepubliceerde reviews kwamen tot dezelfde conclusies.

Er is minder onderzoek gedaan naar de effecten van mentale training bij patiënten met de ziekte van Parkinson: slechts twee studies. In een onderzoek naar micrografie (steeds kleiner schrijven) bleek mentale training geen effect op de lettergrootte van het handschrift te hebben. In het andere onderzoek, naar bradykinesie (bewegingstraagheid), bleken patiënten die mentale training toepasten wel gemakkelijker te bewegen.

\section{Ontwikkelen en testen van materiaal ter voorbereiding van de klinische studies}

Ter voorbereiding van de klinische studies is er (1) onderzocht of mentale representaties van bewegingen gevisualiseerd konden worden, (2) is er een mentale training interventie ontwikkeld en (3) is zorgvuldig gekozen voor meetinstrumenten om mogelijke effecten te meten.

\section{Het visualiseren van bewegingsvoorstellingen met een computer programma}

Voordat een patiënt gevraagd wordt om buiten de therapie om mentaal te trainen, moet eerst de techniek van het voorstellen geoptimaliseerd worden. Hierbij zijn twee aspecten van belang. Enerzijds moeten alle componenten van een beweging voorgesteld kunnen worden in de juiste volgorde. Anderzijds moet de mentale representatie zo levendig mogelijk zijn door het koppelen van sensorische informatie aan de visuele bewegingsvoorstelling.

Veel patiënten hebben na een beroerte moeite met het plannen en uitvoeren van bewegingen. Dit kan aan de fysieke beperkingen liggen, maar kan ook komen doordat de motorische blauwdruk in de hersenen 'beschadigd' is. Het lijkt niet zinvol om patiënten bewegingen te laten voorstellen waarvan het verloop niet tot een succesvolle uitvoering kan leiden. De eenvoudigste manier om erachter te komen of de patiënt weet uit welke stappen een beweging bestaat, is deze te bevragen. In de sport wordt hiervoor soms een computerprogramma gebruikt: de 'Structural Dimension Analysis of Motor memory' (SDA-M). Bewegingen worden opgeslagen als representaties in het lange termijn geheugen in de vorm van zogenaamde 'basic action concepts' (BACs). BACs zijn deelbewegingen of componenten van een beweging die essentieel zijn voor een succesvolle bewegingsuitvoering. Voorbeelden van BACs voor het 'drinken uit een kopje' zijn: het kopje vastpakken, het kopje aan de lippen aanzetten, het kopje kantelen. Bij afname van de 
SDA-M worden op een scherm op basis van toeval twee BACs getoond, waarbij de proefpersoon moet aangeven of deze twee componenten een nauwe relatie met elkaar hebben tijdens de bewegingsuitvoering of niet. Deze splitprocedure wordt herhaald totdat alle foto's met elkaar vergeleken zijn. Het resultaat wordt weergegeven in een boomdiagram. Hiaten of problemen in de bewegingsafloop kunnen zo opgespoord worden.

Uit onze studie met de SDA-M bleek dat het meetinstrument hanteerbaar was binnen de populatie patiënten na een beroerte. De uikomsten en de interpretatie van de boomdiagrammen waren bruikbaar in de praktijk om mentale training maatgesneden, op individuele basis aan te bieden en te verbeteren. De keerzijde van het gebruik van de SDA-M was, dat afname van de test lang duurde en dat er relatief weinig bekend is over de validiteit van de SDA-M (methodologische kwaliteit). Bovendien is het moeilijk om de resultaten uit de boomdiagrammen (voor een groep) te kwantificeren. Hierdoor werd vergelijking van de resultaten uit de SDA-M met andere meetinstrumenten bemoeilijkt dan wel onmogelijk.

\section{Het toepassen van mentale training: ontwikkeling van een interventie}

Bij het uitwerken van een mentaal trainingsprogramma is uitgegaan van sportliteratuur, evidence vanuit de richtlijnen en reviews op het gebied van revalidatie. Uit de multi- en monodisciplinaire richtlijnen voor de behandeling van CVA-patiënten komt naar voren dat algemene trainingsprincipes die bekend zijn uit de sport ook binnen de revalidatie gelden. Zo dienen bijvoorbeeld door de patiënt gekozen taken (hulpvraag) contextgebonden en functioneel geoefend te worden. En ook voorwaardelijkheden zoals mobiliteit en kracht, moeten in de revalidatie gecreëerd worden. En, hoe meer er binnen de fysiologische grenzen van vermoeidheid en adaptatie getraind wordt, hoe groter de effecten zijn.

De techniek moet gebruikt kunnen worden door fysiotherapeuten en ergotherapeuten en door zo veel mogelijk patiënten met een CVA uitgevoerd kunnen worden, het liefst in meerdere settings. Mentale training is een complexe interventie waarbij maatwerk vereist is: patiënten hebben verschillende niveaus van functioneren en verschillende hulpvragen waaraan ze willen werken. Bij de ontwikkeling van een mentaal trainingsprogramma is daarom gekozen voor een theoretisch kader, waarin plaats is voor individuele aanpassingen. De interventie duurde zes weken.

Het theoretische raamwerk om mentale training toe te passen, was opgedeeld in vijf stappen (fig. 2 op de volgende pagina). De eerste stap dient om de mentale capaciteit van de patiënt te beoordelen en hierdoor de 'juiste' patiënt te selecteren. De volgende twee stappen zijn erop gericht dat de patiënt ook daadwerkelijk de juiste techniek aangeleerd krijgt en uitvoert. 
Zodra de patiënt de techniek beheerst kan het mentale trainen ook zonder supervisie van de therapeut plaatsvinden. Uiteraard moet de imagery techniek in gelijke tred verbeterd worden met veranderingen in de motoriek (stap 4). Tijdens het motorische herstel wordt tijdens contactmomenten met de therapeut dus ook aandacht besteed aan het verbeteren van de mentale vaardigheden. De laatste stap is niet voor elke patiënt weggelegd, maar indien mogelijk is het zinvol de techniek te generaliseren naar de thuissituatie en andere taken.

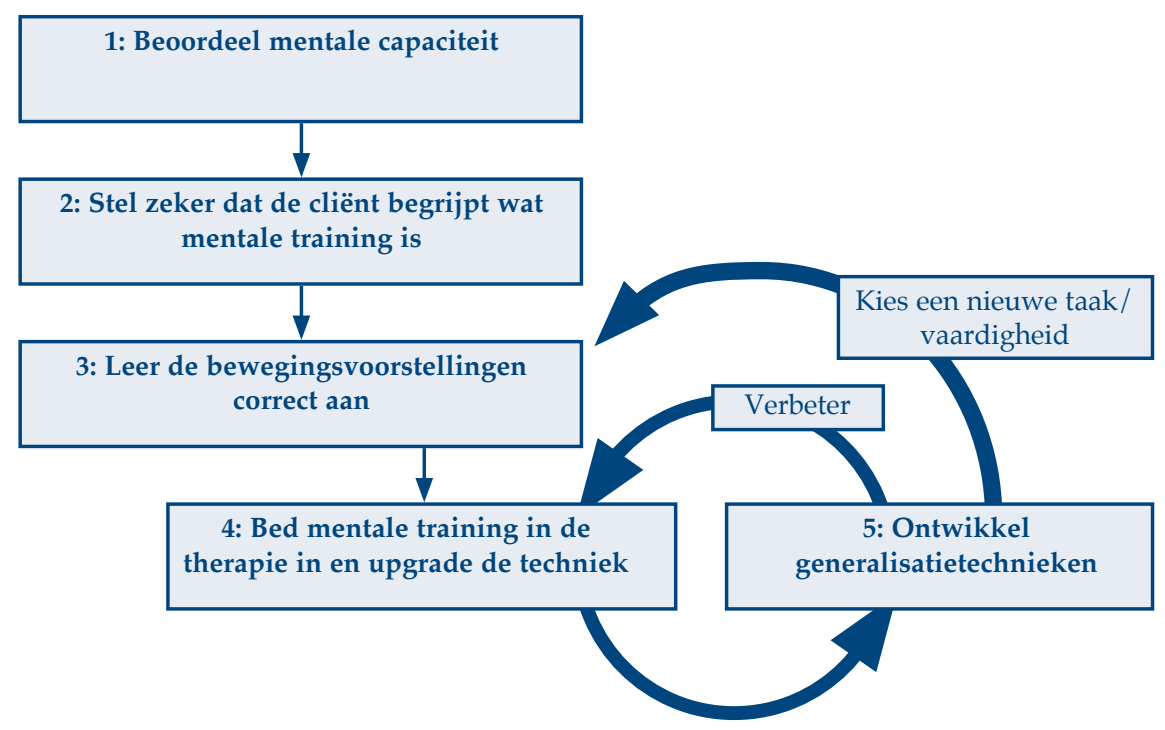

Fig. 2: Overzicht van de stappen uit het mentale trainingsprogramma.

\section{Het bepalen van de meetinstrumenten}

De medische onderzoeksraad (MRC) en verschillende nationale richtlijnen voor de behandeling van patiënten na een beroerte of met de ziekte van Parkinson bevelen meetinstrumenten aan om verandering in het functioneren van de patiënten te meten. Bij het bepalen van de meetinstrumenten in de klinische studies speelden volgende factoren een rol: (1) de meetinstrumenten moesten passen bij de onderzoeksvraag; (2) van voldoende methodologische kwaliteit zijn; en (3) het liefst aanbevolen worden door een nationale behandelrichtlijn. Hierdoor werd ervoor gekozen mogelijke effecten door een testbatterij te meten.

In het voortraject is het ontwikkelde en gekozen materiaal getest in een hanteerbaarheidstudie en zijn enkele case studies uitgevoerd. Naar aanleiding van deze pilotstudies zijn enkele kleine veranderingen doorgevoerd en is er gestart met de gerandomiseerde klinische studies. 


\section{Deel 2: Evaluaties van de klinische studies}

De onderzoeksvragen en de onderzoeksmethode

Er zijn binnen dit promotieonderzoek twee klinische studies uitgevoerd waarbij mentale training vergeleken werd met een controle groep: één bij mensen na een beroerte en één bij mensen met de ziekte van Parkinson. De toekenning van een patiënt tot de controle of de mentale training groep was gebaseerd op toeval (loting). In beide gevallen luidde de onderzoeksvraag:

Wat zijn de effecten van mentale training op het lichamelijk functioneren van de patiënt met een verworven neurologische aandoening?

Bij de patiënten na een beroerte wilden wij weten of mentale training in de experimentele groep zou leiden tot een versneld of vergroot motorisch herstel. Dit motorisch herstel werd gemeten bij het ' lopen' en het 'drinken' en bij twee andere, zelfgekozen activiteiten.

Bij patiënten met de ziekte van Parkinson wilden wij achterhalen of motorische achteruitgang zou kunnen worden tegengegaan of het motorische niveau misschien zelfs licht verbeteren, door het toepassen van mentale training in de experimentele groep. De motorische prestatie van het lopen of een transfer werd beoordeeld.

De klinische trial voor patiënten met een beroerte Aan dit onderzoek konden de bewoners van drie verpleeghuizen meedoen: verpleeghuis Klevarie, verpleeghuis St. Camillus en verpleeghuis Sevagram. In het totaal werden 391 patiënten, waarvan de beroerte niet langer dan 10 weken geleden gebeurd was, gescreend voor deelname aan het onderzoek. Hiervan werden 65 patiënten geschikt bevonden voor het onderzoek en hebben er 36 uiteindelijk toestemming gegeven en zijn ingesloten in de studie (tabel 1 op de volgende pagina). 
Tab. 1: Kenmerken van de patiënten uit de controle en de experimentele groep in de studie met patiënten na een beroerte.

\begin{tabular}{lll} 
Kenmerken & Controle groep & $\begin{array}{l}\text { Experimentele } \\
\text { groep } \\
\mathrm{n}=18\end{array}$ \\
\hline Leeftijd: gemiddelde (SD) & $\mathrm{n}=18$ & $77,7(7,2)$ \\
Tijd na optreden beroerte in weken: gemiddelde (SD) & $77,9(7,4)$ & $6,1(2,7)$ \\
Barthel Index & $12,2(5,4)$ & $11,2(4,1)$ \\
Geslacht: aantal (\% mannen/vrouwen) & $9 / 9(50 \% / 50 \%)$ & $5 / 13(27,8 \% / 72,2 \%)$ \\
MMSE: gemiddelde (SD) & $24,8(4,6)$ & $25,4(3,6)$ \\
Aangedane hersenhelft: aantal (\%-aandeel) & & \\
$\quad$ Links & $9(50 \%)$ & $6(33,3 \%)$ \\
$\quad$ Rechts & $8(44,4 \%)$ & $9(50 \%)$ \\
$\quad$ Centraal/stam & $1(5,6 \%)$ & $3(26,7 \%)$ \\
Vermogen tot bewegingsvoorstellingen (n=17): & & \\
aantal (\%-aandeel) & & $2(11,8 \%)$ \\
$\quad$ Niet in staat & $1(5,9 \%)$ & $7(41,2 \%)$ \\
$\quad$ Een beetje in staat (vage beelden) & $6(35,3 \%)$ & $5(29,4 \%)$ \\
$\quad$ Gemiddeld in staat & $3(17,6 \%)$ & $3(17,6 \%)$
\end{tabular}

SD: Standaard deviatie | MMSE: Mini Mental State Examination

Vermogen om bewegingsvoorstellingen te generen: inschatting door de patiënt zelf

\section{Behandelingen}

De behandeling in de experimentele groep bestond uit de reguliere therapie volgens de nationale multidisciplinaire richtlijnen van de Nederlandse Hartstichting en de nationale richtlijnen van de beroepsverenigingen met in zo veel mogelijk behandelingen ingebedde mentale training.

De behandeling in de controle groep bestond uit de reguliere therapie volgens de nationale multidisciplinaire richtlijnen van de Nederlandse Hartstichting en de nationale richtlijnen van de beroepsverenigingen. Er werd gecorrigeerd voor aandacht door dezelfde metingen uit te voeren en ook de patiënten uit de controle groep een dagboekje bij te laten houden.

\section{Meten van het effect}

Om effecten van mentale training te meten werden alle patiënten op dezelfde momenten gemeten: bij begin van de studie (T0 voor de interventie), na zes weken interventie (T1 korte termijn effecten) en na zes maanden (T2 lange termijn effecten). De scores op T1 en T2 werden vergeleken met de beginscore en er werd bepaald of de mentale traininggroep meer of sneller vooruitging dan de patiënten uit de controle groep. 
De primaire uitkomstmaat was een 10 puntsschaal $(1=$ heel slecht en $10=$ uitstekend $)$ waarop de patiënten konden aangeven in hoeverre het uitvoeren van het lopen en drinken en twee zelf gekozen activiteiten op dat moment ging. De behandelende therapeuten beoordelen dit ook met behulp van deze 10 puntsschaal. Andere (secundaire) uitkomstmaten beoordeelden verandering in functies en activiteiten met gestandaardiseerde en erkende meetinstrumenten: Motricity Index, Berg Balance Scale, Rivermead Mobility Index, Nine Hole Peg Test, Barthel Index en de 10 meter looptest.

Er werd een analyse van data gedaan waarbij er rekening gehouden wordt met een afhankelijkheid van de data met de tijd, de zogenaamde generalized estimating equations (GEE). Het is een vorm van regressieanalyse met een longitudinaal karakter.

In eerste instantie werden de groepen geanalyseerd zoals ze toegekend waren door de loting (intention to treat analyse). Vervolgens werd er gekeken wat de mensen uit de verschillende groepen daadwerkelijk in de therapie gedaan hebben. Patiënten uit de mentale training groep, die niet (voldoende) mentaal geoefend hadden en patiënten uit de controle groep die juist wel mentaal geoefend hadden, werden uit de dataset gehaald. Nu werd de analyse herhaald als een zogenaamde per protocol analyse.

\section{Resultaten}

Beide groepen hadden een vergelijkbare hoeveelheid therapie. Sommige patiënten uit de groep die mentale training kreeg, werden in het begin (om mentale training aan te leren) iets meer dan gemiddeld behandeld. In beide groepen werden dagboekjes bijgehouden en interviews gehouden, waarin de patiënten gevraagd werden naar hun meningen betreffende de behandeling.

Beide groepen gingen tijdens het onderzoek enorm vooruit. De meeste vooruitgang werd gezien bij de eerste meting (T1 na zes weken interventie). Echter, de verschillen tussen de groepen waren klein. In deze studie konden we dan ook niet vaststellen dat mentale training een effect heeft op het sneller of beter fysiek herstellen van patiënten met een beroerte in de sub acute fase van herstel, niet na de zes weken interventieperiode en ook niet na de zes maanden follow-upmeting. Figuur 3 op de volgende pagina geeft een voorbeeld van het herstel van de patiënten, in dit geval gemeten met de Barthel Index. Te zien is dat in beide groepen de patiënten vooruit gaan en dat de verschillen onderling minimaal zijn. Bij alle andere meetinstrumenten werden soortgelijke resultaten gevonden. 


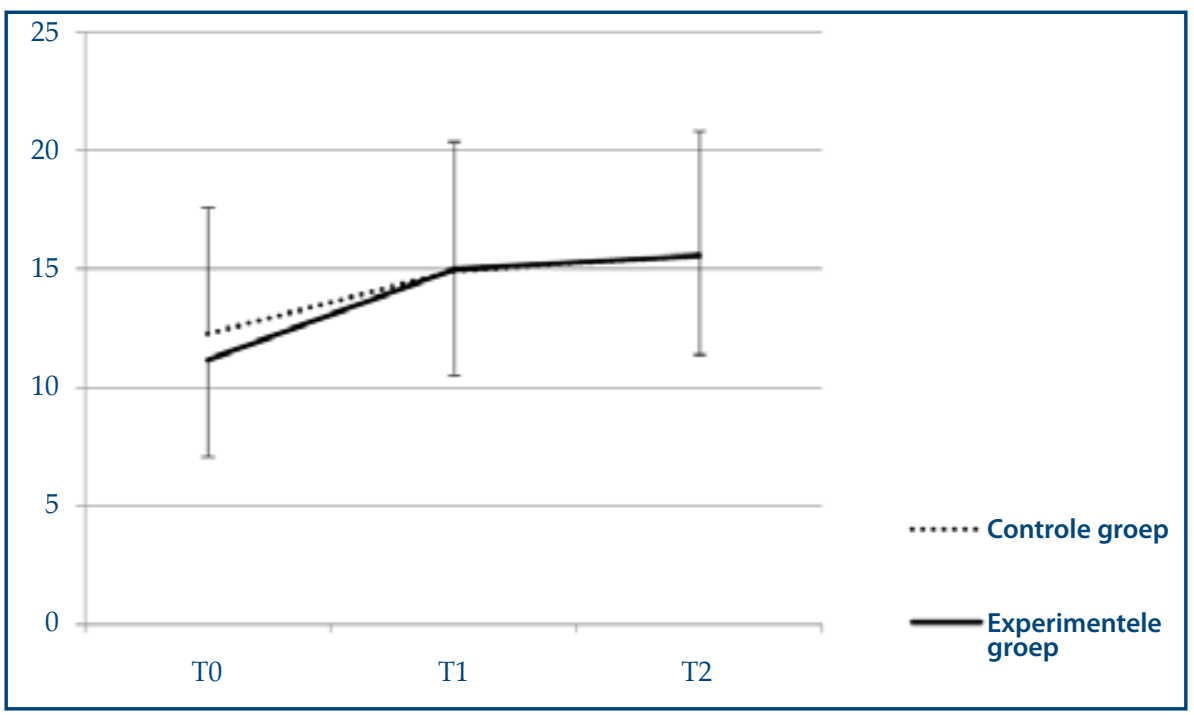

Fig. 3: Resultaat van de metingen met de Barthel Index tijdens het onderzoek (T0 = voormeting । $\mathrm{T} 1=$ na de zes weken interventieperiode $\mid \mathrm{T} 2=$ bij de nameting na zes maanden)

\section{Discussie en conclusie}

In tegenstelling tot het gros van de publicaties op het gebied van mentale training als additionele interventie in de revalidatie van mensen na een beroerte, vonden wij in deze studie geen positieve effecten. Drie mogelijke redenen hiervoor worden bediscussieerd: de onderzoekspopulatie, de interventie en de uitkomstmaten inclusief 'power'.

In Nederland wordt het merendeel van de mensen die een beroerte krijgen, behandeld in een verpleeghuis en dus leek het belangrijk om juist deze groep patiënten te onderzoeken.

Echter, het bleek moeilijk om patiënten te includeren in het onderzoek: minder dan $10 \%$ wilde of kon meedoen. Waarschijnlijk hebben we ook een groep patiënten gemist, die geschikt zou zijn voor deze therapievorm, maar die te kort in het verpleeghuis verblijft om deel te kunnen nemen. Het zijn patiënten die binnen enkele weken naar huis gaan of overgedragen worden aan een gespecialiseerd revalidatiecentrum. De groep die overbleef en meegedaan heeft aan dit onderzoek was uiteindelijk een hele kenmerkende (specifieke) en kwetsbare sub groep, wat voor een deel de resultaten zou kunnen verklaren.

Een tweede reden waarom wij geen effecten gevonden hebben, hangt met de interventie samen. Het aanleren en toepassen van mentale training is een complexe onderneming, zeker als patiënten met een complexe aandoening dit moeten doen, binnen een complexe zorgsituatie. 
Bovendien was de interventie geconstrueerd als een stappenplan, een kader waarbinnen therapeuten en patiënten keuzes konden maken, zodat de interventie meer maatgesneden kon plaatsvinden. Enerzijds is dit motiverend voor de patiënt en nodig om een complexe interventie op maat te kunnen aanbieden. Anderzijds is deze manier van werken 'onzeker' voor de therapeut. Wellicht dat een wat meer praktijkgerichte, pragmatische manier van werken de voorkeur van de therapeuten had gehad. De mogelijkheden om de interventie individueel vorm te geven, heeft er ook toe geleid, dat er verschillen waren in de inhoud en hoeveelheid therapie die patiënten hebben gekregen. Ondanks dat het merendeel van de patiënten buiten de therapie met de therapeuten om tot 30 uur (in zes weken) zelf, onafhankelijk geoefend heeft, kan het toch zijn, dat dit onvoldoende was om een extra, meetbaar effect te krijgen.

Hiermee komen we bij de laatste reden: de meetinstrumenten en de bewijskracht. Ondanks dat een groot deel van de populatie na een beroerte een lange en moeilijke revalidatieperiode tegemoet gaan, herstellen veel patiënten gelukkig in meer of mindere mate wel weer. Het herstel is een combinatie van natuurlijk herstel (wondgenezing) en een multidisciplinaire behandeling. Dit herstel is meetbaar met alle meetinstrumenten die in deze studie gebruikt zijn. Echter, het herstel is wellicht zo groot, dat de potentiële meerwaarde van mentale training binnen de vorderingen niet te meten is. Daarbij komt dat er in onze studie te weinig mensen meegedaan hebben (te weinig power). Misschien zou het additionele effect wel te vinden zijn, indien de groep groter zou zijn. Daarnaast hebben wij alleen gekeken naar effecten van mentale training op het lichamelijke functioneren, terwijl mentale training misschien juist effect heeft op het veranderen van cognitie en emoties. Deze 'zachte' effecten zijn vaker gerapporteerd door atleten in de sport en ook binnen onze studies vertelden patiënten vaker, dat zij door het gebruik van mentale training ook een gevoel van autonomie kregen, een gevoel dat zij meer grip kregen op hun revalidatieproces.

Op basis van de resultaten van deze studie kunnen wij het gebruik van mentale training als onderdeel van de standaardzorg binnen de revalidatie van patiënten met een beroerte niet bij alle patiënten aanbevelen. Echter, mentale training zou bij bepaalde patiënten kunnen helpen en wij moedigen het gebruik van mentale training bij individuele patiënten die mentaal willen oefenen dan ook aan, vooral in het kader van onderzoek. Het beter kunnen bepalen wie er mogelijk baat bij heeft, moet verder onderzocht worden (prognostisch onderzoek). Bovendien is meer onderzoek nodig op het gebied van het ontwikkelen en toetsen van mentale training interventies. De interventie zoals hier beschreven zou ook bij een specifieke andere subgroep verder onderzocht kunnen worden. 


\section{De klinische trial voor patiënten met de ziekte van Parkinson}

Naar aanleiding van vragen uit het werkveld, wilden wij bij patiënten met de ziekte van Parkinson achterhalen of de beleving van de kwaliteit van de motoriek en de prestatie van het lopen of een transfer beïnvloed zou kunnen worden door het toepassen van mentale training in de experimentele groep. Er werd een controle groep toegevoegd waarin ontspanningstherapie werd gegeven. Of iemand in de controle of experimentele groep kwam, werd op basis van toeval bepaald (per locatie werden er lootjes getrokken). We waren er ook in geïnteresseerd of patiënten in een eerder stadium van de ziekte (Hoehn en Yahr lager dan 3) anders reageren op het gebruik van mentale training dan wel ontspanningstherapie dan de gehele groep, met andere woorden of zij meer of minder geschikt zijn voor één van deze therapievormen.

Aan dit onderzoek deden patiënten met de ziekte van Parkinson mee, die behandeld werden in de eerste, tweede en derde lijn. Vijf settings deden mee aan het werven van patiënten: drie particuliere praktijken (fysiotherapiemaatschap Snijders, Fysiovision en fysiotherapie de Baandert) en de afdeling polikliniek fysiotherapie van het ziekenhuis Orbis medisch centrum en het verpleeghuis St. Camillus. In de periode van zes maanden werden 59 patiënten gescreend voor deelname waarvan er 54 geschikt waren voor het onderzoek en 47 patiënten vrijwillig instemden met deelname (tabel 2 op de volgende pagina).

\section{Behandelingen}

De behandeling in de experimentele groep bestond uit de reguliere therapie volgens de nationale richtlijnen fysiotherapie van het Koninklijk Nederlands Genootschap voor Fysiotherapie (KNGF) met in zo veel mogelijk behandelingen ingebedde mentale training.

De behandeling in de controle groep bestond uit de reguliere therapie volgens de nationale richtlijnen fysiotherapie van het Koninklijk Nederlands Genootschap voor Fysiotherapie (KNGF) met in zo veel mogelijk behandelingen ingebedde ontspanningsoefeningen. In tegenstelling tot de studie met de patiënten na een beroerte werd ervoor gekozen om een interventie in de controle groep toe te voegen. De belangrijkste reden was, om de extra aandacht gelijk te houden door in beide groepen een additionele interventie aan te bieden, alle deelnemers dagboekjes bij te laten houden, dezelfde metingen uit te voeren op dezelfde tijdstippen, en deelnemers uit beide groepen te interviewen. In de enige vergelijkbare andere studie waarin effecten van mentale training in deze doelgroep werden onderzocht, werd in de controle groep ook ontspanningstherapie gegeven. Wij hebben deze 'controle-interventie' overgenomen, zodat resultaten vergeleken konden worden. 
Tab. 2: Patiëntkenmerken van de gehele groep (links) en van de subgroep patiënten met de mildere symptomen van de ziekte van Parkinson (Hoehn en Yahr <3), beide keren opgedeeld voor de controle en de experimentele groep.

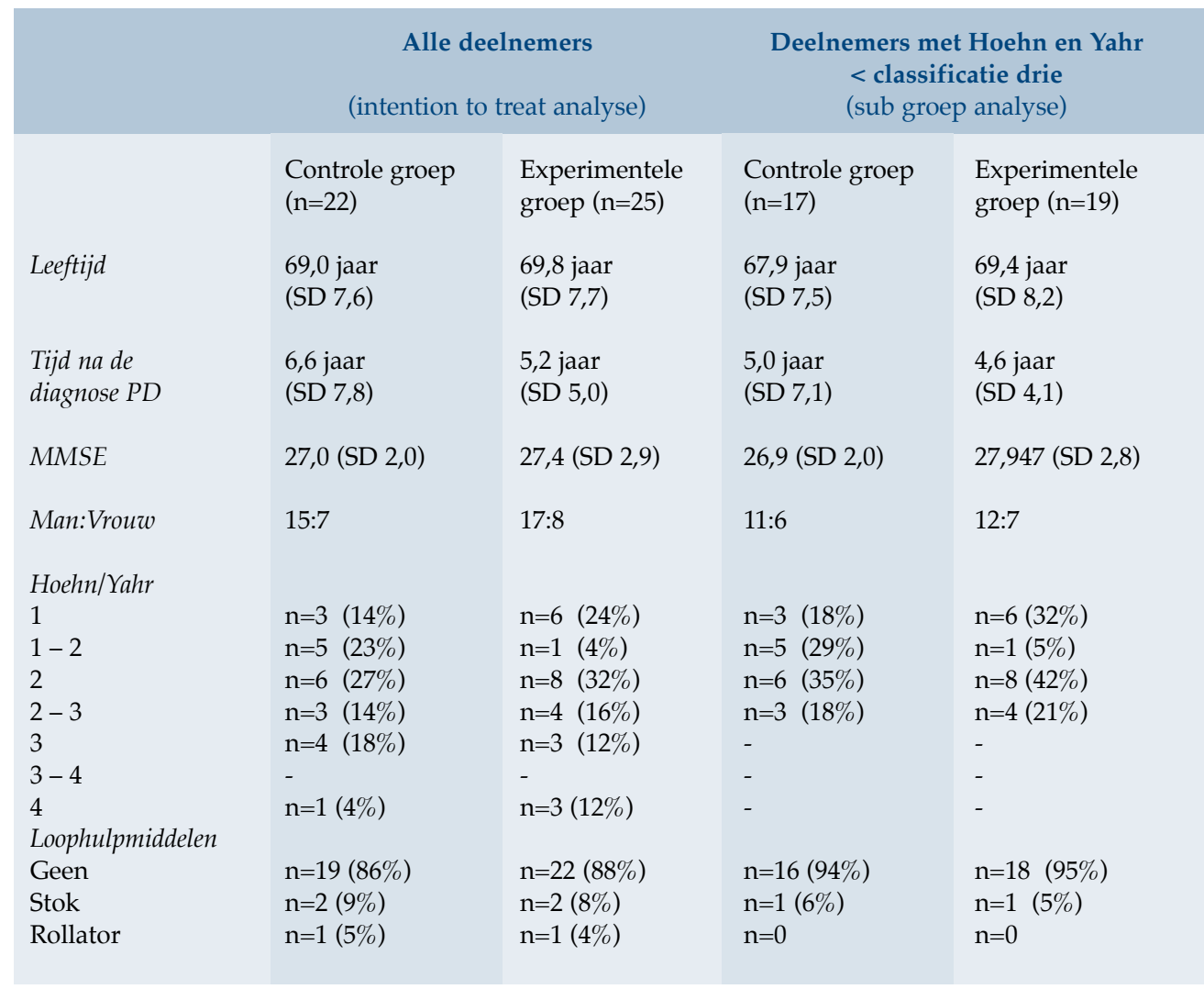

$P D=$ Parkinson disease (ziekte van Parkinson) $\mid M M S E=$ Mini Mental State Examination $\mid S D=$ Standaard deviatie

\section{Meten van het effect}

Om effecten van mentale training te meten en te vergelijken met de controle groep werden alle patiënten op dezelfde momenten gemeten: bij begin van de studie (T0 voor de interventie), na zes weken interventie (T1 korte termijn effecten) en na drie maanden (T2 lange termijn effecten). De scores op T1 en T2 werden vergeleken met de beginscore (per groep) en er werd bepaald of de mentale traininggroep minder achteruit ging dan of misschien zelfs wel stabiel bleef of vooruit ging vergeleken met de patiënten uit de controle/ontspanningsgroep.

Er werden vijf uitkomstmaten gebruikt om effecten te meten. Net als in de studie met patiënten met een beroerte was een van de uitkomstmaten de subjectieve inschatting van het gaan of van 
een transfer. In plaats van een 10 puntsschaal werd een lijntje van $10 \mathrm{~cm}$ gebruikt om op aan te geven hoe het lopen op dat moment werd uitgevoerd $(0=$ heel slecht en $10=$ uitstekend). De behandelende therapeuten beoordelen dit ook met behulp van deze 10cm-lijn (visual analogue scale; VAS). De andere uitkomstmaten beoordeelden verandering in de mobiliteit van de patiënt met volgende meetinstrumenten: timed up and go, de 10 meter looptest en (indien mogelijk) de tijd die nodig is om vanuit staande positie plat te gaan liggen en weer te gaan staan.

Er werd een analyse van data gedaan waarbij er rekening gehouden wordt met een afhankelijkheid van de data met de tijd, de zogenaamde generalized estimating equations (GEE). Het is een vorm van regressieanalyse met een longitudinaal karakter.

In eerste instantie werden de groepen geanalyseerd zoals ze toegekend waren door de loting (intention to treat analysis). Vervolgens werd er gekeken wat de mensen uit de verschillende groepen daadwerkelijk in de therapie gedaan hebben. Patiënten uit de mentale training groep, die niet mentaal geoefend hadden maar wel ontspanningsoefeningen en patiënten uit de controle groep die juist wel mentaal geoefend hadden of geen ontspanningsoefeningen gedaan hadden, werden uit de dataset gehaald. $\mathrm{Nu}$ werd de analyse herhaald (per protocol analyse).

Als laatste werd intention to treat analyse herhaald met de subgroep patiënten die een Hoehn en Yahr classificatie hebben onder de 3.

\section{Resultaten}

Beide groepen hadden een vergelijkbare hoeveelheid therapie, begeleid door een therapeut, en hebben bijna gelijk veel zelfstandig geoefend.

Beide groepen gingen meestal vooruit op de tests (na de zes weken interventie). Bij sommige metingen was dit effect nog steeds te zien na drie maanden (T2 nameting). Echter, het voordeel was soms voor de controle groep en soms voor de experimentele en het verschil tussen de groepen was klein (statistisch niet significant). Dit gold zowel voor de intention to treat analyse als voor de per protocol analyse

Indien alleen de patiënten in de eerste fasen van het ziektebeeld geanalyseerd werden (Hoehn en Yahr score kleiner dan 3), dan was er een trend zichtbaar in het voordeel van de mentale training groep (figuur 4 en 5). 


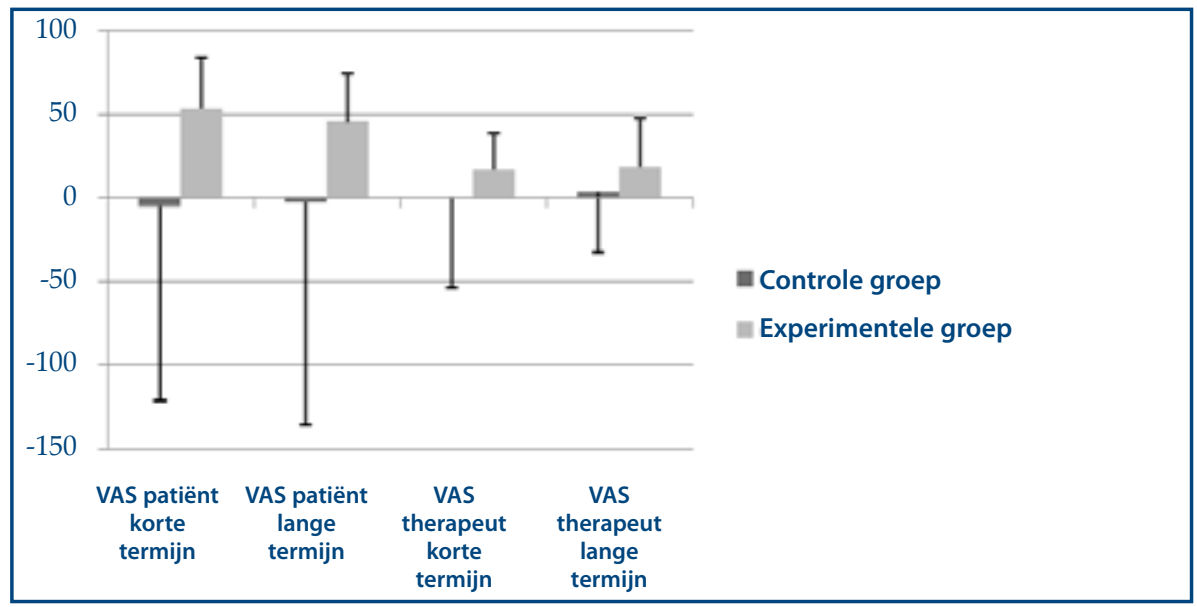

VAS = visual analogue scale (10puntsschaal), waarbij een positieve verandering een verbetering is.

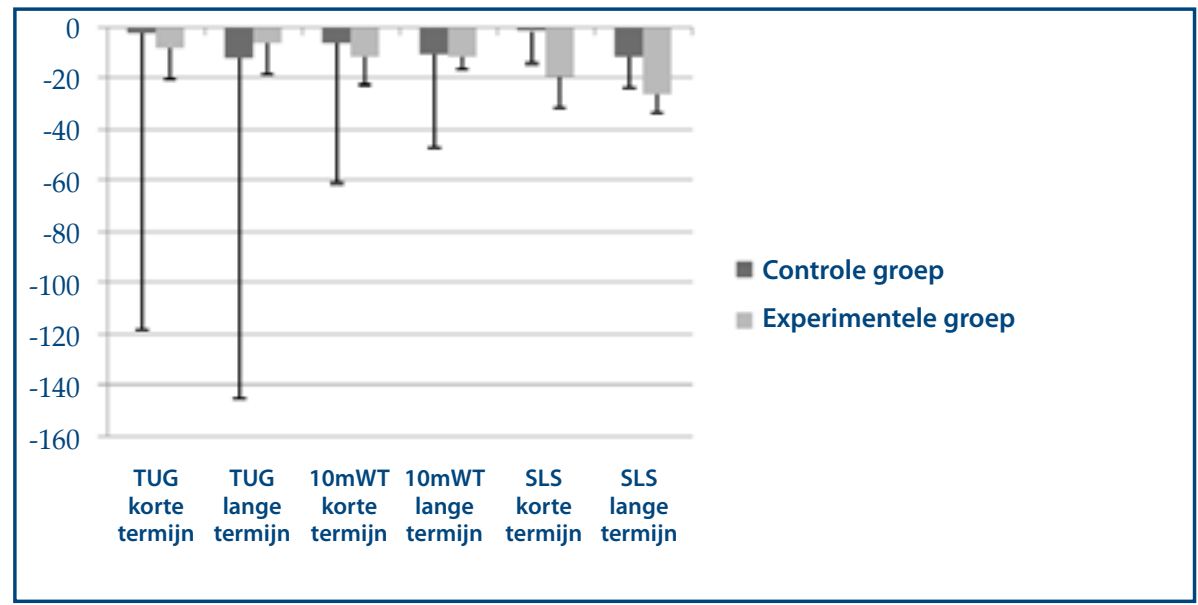

TUG=Timed $U p$ and Go $\mid 10 \mathrm{~m} W T=10 \mathrm{~m}$ looptest $\mid$ SLS = tijd die nodig is om vanuit staande positive te gaan liggen en weer op te staan; staan-liggen-staan. Aangezien het bij de drie metingen om tijden gaat (hoe sneller, hoe beter), is een negatieve verandering een verbetering.

Fig. 4 en 5: Resultaten van de sub groep analyse (Hoehn en Yahr <3) voor de controle en experimentele groep (vergeleken): gemiddelde verandering (incl. SD) uitgedrukt in percentages (\%) wat betreft de VAS score zoals beoordeeld door de patiënt en de therapeut (eerste figuur), de Timed up and go (TUG), de 10 meter looptest (10mWT) en de tijd die nodig is voor staan-liggen-staan (SLS) score (tweede figuur) vergeleken met de voormeting $((\mathrm{T} 1-\mathrm{T} 0) / \mathrm{T} 0 * 100$ na de zes weken interventieperiode; korte termijn effecten en ( T2-T0)/T0*100\% bij de nameting; lange termijn effecten). 


\section{Discussie en conclusie}

In tegenstelling tot de enige vergelijkbare studie met patiënten met de ziekte van Parkinson hebben wij geen positieve effecten van mentale training gevonden. Mentale training zoals het in deze studie is toegepast lijkt dan ook geen meerwaarde te hebben voor de gehele groep patiënten met de ziekte van Parkinson. Indien alleen de patiënten in de mildere fasen van het ziektebeeld worden bekeken, lijken de patiënten uit de mentale training groep het beter te doen dan de patiënten uit de ontspanningsgroep op alle meetinstrumenten en beide meetmomenten, m.u.v. de TUG bij de nameting. Dit voordeel is echter niet groot genoeg om statistisch significant te zijn. Een reden zou kunnen zijn, dat de onderzochte groep in deze studie niet groot genoeg was (onvoldoende power bij de subgroep). Misschien hadden we het effect van mentale training in een grotere subgroep wel kunnen aantonen.

Het verschil tussen de groepen was ook relatief klein doordat beide interventies onverwacht een positief effect hadden. Dit is nog opmerkelijker omdat de ziekte van Parkinson een degeneratief ziekteverloop heeft.

Dezelfde vraagstekens omtrent de mentale trainingsinterventie die we bij de populatie met een beroerte hadden, hebben we hier ook: er zullen door de flexibiliteit van de mentale trainingsinterventie verschillen zijn tussen personen binnen de experimentele groep wat betreft de inhoud en omvang van het mentaal oefenen. Misschien was de mate van oefenen van sommige patiënten onvoldoende om effecten voor de gehele groep aan te tonen.

Op dit moment kan op basis van de resultaten van deze studie niet aanbevolen worden om de mentale training zoals hij hier aangeboden is, standaard te gebruiken binnen de behandeling van patiënten met de ziekte van Parkinson. Echter, mentale training zou bij bepaalde patiënten kunnen helpen en meer onderzoek is noodzakelijk. Toekomstig onderzoek zou nog beter effecten van mentale training moeten differentiëren bij patiënten die zich bevinden in de mildere en meer ernstige fasen van de ziekte van Parkinson. Wellicht dat de patiënten die zich bevinden in Hoehn en Yahr fase 2,5 of lager meer geschikt zijn om mentale training te leren en hier dan misschien ook meer baat bij hebben (ook op langere termijn).

Ervaringen van patiënten na een beroerte of met de ziekte van Parkinson met het gebruik van mentale training

Om meer inzicht te krijgen in de beleving en ervaring van de patiënten met betrekking tot mentale training werd ook een procesevaluatie uitgevoerd. Als onderdeel van de procesevaluatie werden in de experimentele groepen individuele en focusgroep interviews afgenomen. Hierin werd onder andere gevraagd waarom patiënten mentaal trainen, welke baat zij van het voorstellen dachten te hebben. 
De patiënten in beide onderzoeken werden aangeleerd mentale training te gebruiken om de motoriek te beïnvloeden door de bewegingen stapsgewijs in gedachten te oefenen. De patiënten uit de CVA-studie bleken echter iets heel anders te doen: ze oefenden vooral op het emotionele en cognitieve vlak. Aspecten van zekerheid, niet bang zijn, jezelf veilig voelen, kwamen herhaaldelijk terug in de interviews. In het merendeel van de geïnterviewde CVA-patiënten bleek naast het beïnvloeden van emoties ook het 'zichzelf motiveren' en aanmoedigen een belangrijk onderdeel van de mentale trainingsinhoud.

In tegenstelling tot de CVA-patiënten gaven veel patiënten met de ziekte van Parkinson juist aan zich volledig op de beweging te concentreren. Het gebruik van mentale training voor het veranderen van emoties, cognitie en motivatie kwamen in de interviews met Parkinsonpatiënten vrijwel niet voor. Er lijkt dan ook een populatiespecifiek aspect aanwezig te zijn waarmee rekening gehouden moet worden als mentale training wordt aangeboden in therapie bij patiënten met een neurologische aandoening.

\section{Conclusie}

Het onderzoek naar het potentiële gebruik van mentale training staat nog in de kinderschoenen. Wij bevelen (nog) niet aan om mentale training grootschalig te implementeren in de zorg van patiënten na een beroerte noch bij patiënten met de ziekte van Parkinson. Wij moedigen het gebruik bij individuele patiënten die graag mentaal willen trainen aan, vooral in de context van onderzoek. Want mentale training zou kunnen helpen.

Onderzoek met grotere populaties zouden zich moeten richten op de vier deelgebieden binnen het ontwikkelen en evalueren van complexe interventies:

\section{Theorie/Modelvorming}

1. Onderzoeken van de onderliggende werkingsmechanismen waarom mentale training werkt bij sommige patiënten en niet werkt bij anderen (op individueel niveau en gerelateerd aan het ziektebeeld).

2. Voorspellen van de geschikte patiënten, het selecteren van de 'juiste' patiënt: bij wie heeft mentale training baat, wie is wel of niet geschikt?

3. Onderzoeken of mentale traininginterventies aangepast moeten worden aan de fase van motorisch herstel, complexiteit van de vaardigheid en specifieke patiëntenpopulaties.

\section{Hanteerbaarheid/Piloting}

4. Bepalen en evalueren van ketenzorg om een meer realistisch beeld te krijgen wat betreft werving van potentiële deelnemers aan onderzoek (CVA).

5. Bepalen van mogelijke negatieve bijwerkingen of effecten van mentale training. 


\section{Evaluatie mentale training}

6. Bepalen van meetinstrumenten en effectmaten in toekomstig onderzoek: tot nu toe is het onderzoek naar de effecten van mentale training in de neurorevalidatie beperkt gebleven tot motorische veranderingen. Het lijkt interessant om ook effecten op het gebied van emoties en cognitie te verkennen en hiervoor de geschikte meetinstrumenten te kiezen.

7. Afstemmen van meetinstrumenten zou vergelijking van resultaten uit verschillende studies mogelijk maken.

8. Gedrag van de patiënten wat betreft mentale training inventariseren met behulp van kwalitatief onderzoek.

9. Evalueren van processen en kosteneffectiviteit.

Op basis van deze informatie kan er bepaald worden of het zinvol is om mentale training te implementeren in de zorg van patiënten met een neurologische aandoening en indien dit het geval is, op welke manier dit moet gebeuren. 


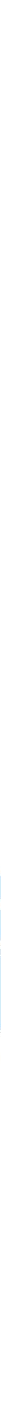




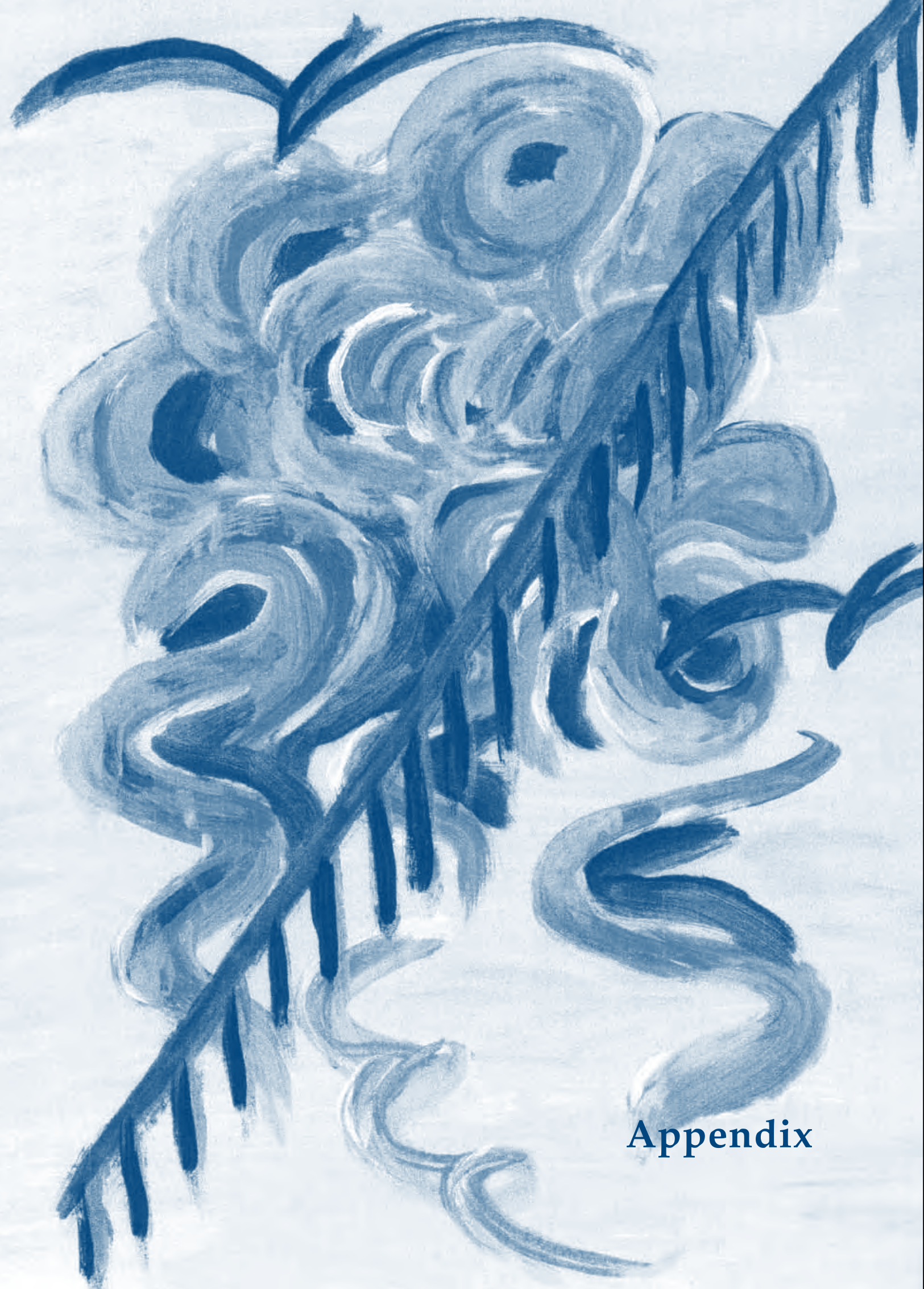




\section{Appendix}

\section{Log example}

Monday, ___ _ -20

How are you feeling today?

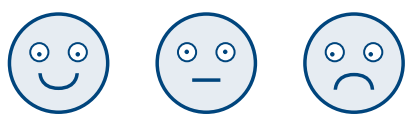

Evaluation of imagery- practice

$\begin{array}{llll}\text { When did } & \text { How long } & \text { Which } & \text { How well did it go? } \\ \text { you practice? } & \text { did you } & \text { activity } & \text { 1: poor } \longrightarrow \text { 10: excellent } \\ \text { Time of the } & \text { practice } & \text { did you } & \\ \text { day } & \text { (minutes)? } & \text { practice? }\end{array}$

$\begin{array}{llllllllll}1 & 2 & 3 & 4 & 5 & 6 & 7 & 8 & 9 & 10\end{array}$

$\begin{array}{llllllllll}1 & 2 & 3 & 4 & 5 & 6 & 7 & 8 & 9 & 10\end{array}$

$\begin{array}{llllllllll}1 & 2 & 3 & 4 & 5 & 6 & 7 & 8 & 9 & 10\end{array}$

$\begin{array}{llllllllll}1 & 2 & 3 & 4 & 5 & 6 & 7 & 8 & 9 & 10\end{array}$

$\begin{array}{llllllllll}1 & 2 & 3 & 4 & 5 & 6 & 7 & 8 & 9 & 10\end{array}$

Any comments: 


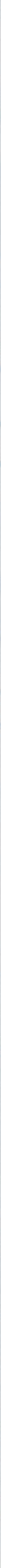




\section{About the author}

Susy was born in February 1971, as the youngest of three children. Her parents, Hermann and Anneliese, emigrated in 1966 from the South of Germany to the United States as a young married couple and settled in Chicago.

Susy had her first education in Breda, the Netherlands. She graduated cum laude from 'het Onze Lieve Vrouwe Lyceum' in Breda (1983-1989). From 1989-1994 she studied movement science at the Sports University of Cologne, Germany (DSHS). She had a job as a student assistant, first at the institute for gymnastics and athletics, later at the institute for biometrics. Her master thesis was on the altered injury patterns in young elite gymnasts. She passed cum laude and was given the Horst Dassler Study Prize for best foreign student in 1995 for her performance at the DSHS. In 1993, she started her education as a physiotherapist at the Zuyd University of applied sciences in Heerlen, the Netherlands. She was one of the founders of student representation. As a side job and hobby she trained young track and field athletes for two years. She graduated cum laude and was nominated for one of the prizes of the Dutch Royal Association for Physiotherapy (KNGF), for her bachelor thesis on clinimetrics in neurological patients.

She started working as a physiotherapist after graduation in 1997, combining practical work as a physiotherapist in the community with teaching at the physiotherapy course of the Zuyd University from May 1998 on. Her first published article on 'the polor own zone' with co-authors Sandra Beurskens, Niels Könntgen and Frank Offermann was rewarded in 2004, when she was given the 'best new coming author of a scientific paper' prize. An initiative by the KNGF. One of her main tasks at the Zuyd University is mentoring students with their bachelor thesis. Together with students she has done various projects with which two national prizes were won. In 2005 she started working part time on her PhD-project on the use of mental practice in neurological patients, which was embedded in the research centre of autonomy and participation of the chronic ill (A\&P) and the centre of expertise in life sciences (CEL) of the Zuyd University of applied sciences and the research centre CAPHRI of Maastricht University. Meanwhile she kept working as a teacher at the department of health and technique (physiotherapy course). She organized three international conferences on neurological rehabilitation together with the Oxford centre for enablement, Oxford, UK.

From 2009 on, she started supervising two larger projects of former students on motor learning. After finishing her $\mathrm{PhD}$, her work will remain a combination of research and teaching.

She lives with her partner and their two beautiful children in the South of Holland. 



\section{Publications}

\section{Mental practice publications}

Braun SM, Beurskens AJ, Borm PJ, Schack T, Wade DT. The effects of mental practice in stroke rehabilitation: a systematic review. Arch Phys Med Rehabil, 2006;66:842-852.

Braun SM, Schack T, Marcellis RG, Oti KC, Schols JM, Beurskens AJ, Wade DT. Is it possible to use the SDA-M to investigate representations of motor actions in stroke patients?

Clin Rehabil 2007;21:822-832.

Braun SM, Beurskens AJHM, van Kroonenburgh S, Demarteau J, Schols JM, Wade DT. Effects of Mental Practice embedded in daily therapy compared to therapy as usual in adult stroke patients in Dutch nursing homes: design of a randomised controlled trial. BMC Neurol 2007;7:34.

Braun SM, Kleynen M, Schols JM, Schack T, Beurskens AJ, Wade DT. Using mental practice in stroke rehabilitation: a framework. Clin Rehabil 2008;22:579-591.

Braun SM. Het gebruik van bewegingsvoorstellingen in de revalidatie: Hoe maak je de transfer van sport naar therapie? In: Jaarboek Fysiotherapie/Kinesitherapie 2009. Geraets J.J.X.R. et al (eds). Houten: Bohn Stafleu van Loghum, 2009. p 191-201.

Kleynen M, Schoenmakers M, Braun SM. Stel je voor, het werkt. Mentale training bij een chronische CVA patiënt: een case report. Ned Tijdschr Fysiother 2009;119:48-54.

Van Elteren D, Vonk J, van Engelen E, Braun SM. De "Structural Dimension Analysis of Motor memory" (SDA-M) in een centraal neurologische revalidatie setting. Stimulus 2009;2:180-197.

Braun SM. Mentale training in de neurologische revalidatie: veranderen van motoriek, cognities en emoties. In: Jaarboek Fysiotherapie/Kinesitherapie 2010. Veenhof C. et al (eds). Houten: Bohn Stafleu van Loghum, 2010. p 244-256. 


\section{Submitted mental practice articles}

Crüts B, Braun SM, Kleynen M, Driessen A, Beurskens AJ, Borm PJ, Wade TD. EEG brain states during motor imagery in stroke patients and healthy controls. (submitted 2009)

Braun SM, van Haastregt JC, Beurskens AJ, Gielen AI, Wade DT, Schols JM. Feasibility of a mental practice intervention in stroke patients in Dutch nursing homes: a process evaluation. (submitted 2009)

Braun SM, Beurskens AJ, Dalemans R, Schols JM, Wade DT. The experience of movement imagery therapy by patients with stroke or Parkinson's disease. (submitted 2010)

Braun SM, Beurskens AJ, Kleynen M, Oudelaar B, Wade DT, Schols JM. Effects of mental practice embedded in daily therapy compared to therapy as usual in adult stroke patients in Dutch nursing homes: results from a multi-centre randomised controlled trial. (submitted 2010)

Braun SM, Beurskens AJ, Kleynen M, Schols JM, Wade DT. Using mental practice in patients with Parkinson's disease: results from a randomised controlled trial. (submitted 2010)

\section{Other publications}

Braun SM, Offermann F, Könntgen N, Beurskens AJHM: De hartfrequentie als maat voor het bepalen van het aërobe trainingsbereik: een onderzoek naar de Own Zone van de Polar M-Serie. Ned Tijdschr Fysiother 2004;114;36-41.

Mey M, Frings K, Braun SM, Rothgangel A. Wii ${ }^{\mathrm{TM}}$ make(s) you move. Die Wii ${ }^{\mathrm{TM}}-$ Konsole in der Behandlung von Patienten mit Morbus Parkinson: eine Patientenserie. Zeitschrift für Physiotherapeuten (accepted 2010)

\section{Submitted other articles}

Rothgangel A, Braun SM, Beurskens AJ, Wade DT. The effects of mirror therapy in rehabilitation: a systematic review. (submitted 2009)

Kleynen M, Braun SM, Verbunt JA, de Bie RA, Masters RS. Psychometric aspects of the Dutch movement specific Reinvestment Scale (D-MSRS). (submitted 2010) 


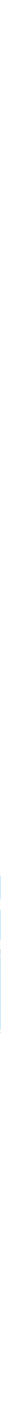




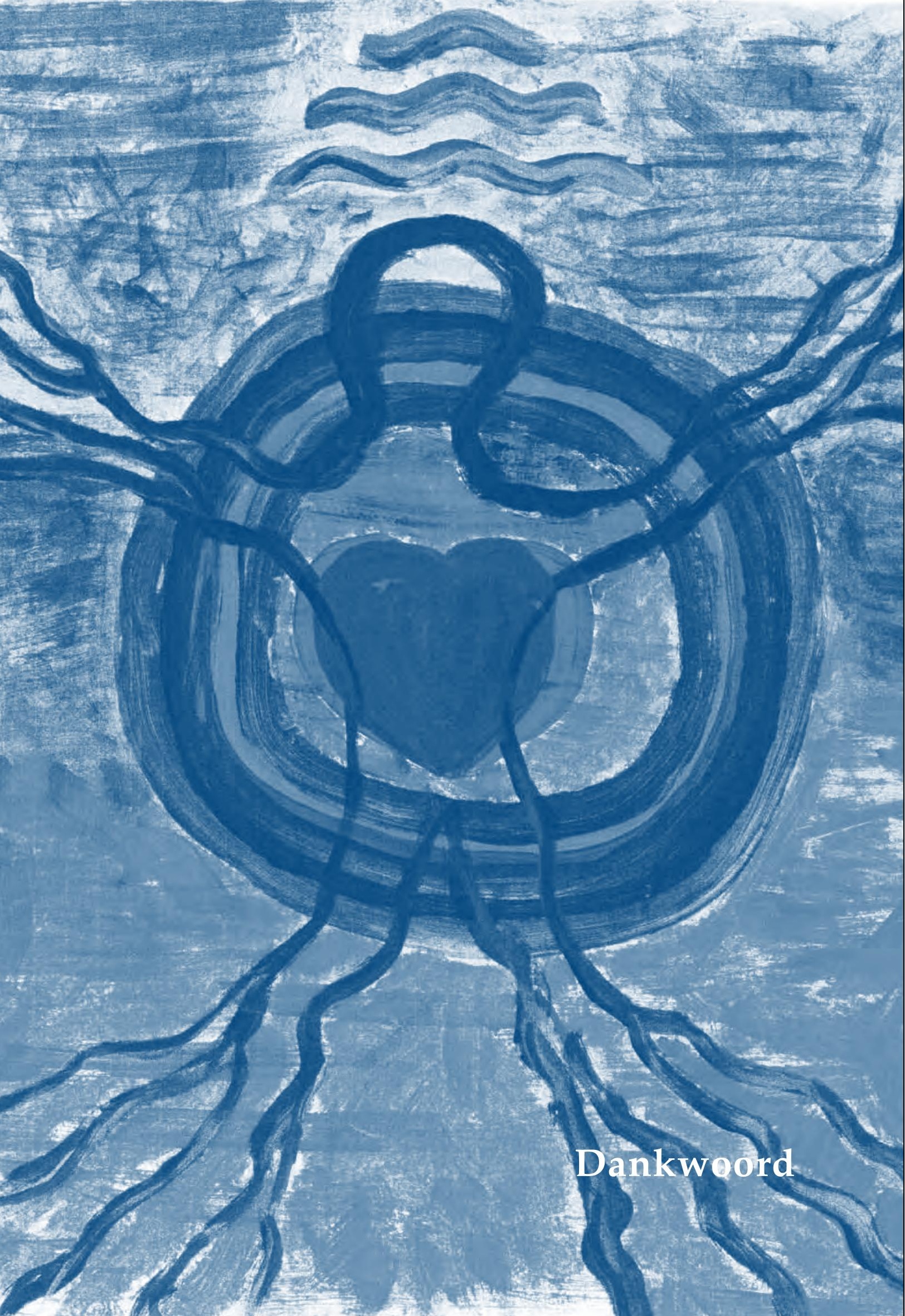




\section{Dankwoord}

In het boek 'Outliers' beschrijft Malcolm Gladwell, dat successen, klein of groot, altijd een combinatie zijn van een aantal factoren. Hij benoemt o.a. een beetje bagage meekrijgen bij je geboorte (IQ schijnt te helpen), je opvoeding (heb je van jongs af aan een aantal vaardigheden geleerd?), heel veel energie ergens in stoppen (gewoon uren maken), in welke tijd je leeft (vrouwen mogen gelukkig tegenwoordig studeren en promoveren), samenloop van omstandigheden (lectoraten zijn in 2004 in het leven geroepen op Hogescholen), kansen zien en grijpen (we gaan gewoon de eerste lijn in met onze ideeën) en de hulp die je van buitenaf krijgt van anderen (zie vervolgtekst). De bottom line is uiteraard bekend: een prestatie is nooit het kennen en kunnen van één persoon. Ik heb veel ondersteuning gehad van veel mensen, af en toe heb ik wat geluk gehad, soms ben ik blind doorgegaan en soms was het idee voor een studie meer een samenloop van omstandigheden, en gek genoeg was het daardoor veelal niet minder goed.

Maar laat ik bij het begin beginnen. Zoals ik al schreef zijn lectoraten een relatief nieuw fenomeen. Ik dank dan ook de leden van het college van bestuur voor het instellen van lectoraten op de Hogeschool Zuyd en het ondersteunen van promovendi. Dhr. Willems, dank ik voor de aanhoudende belangstelling en het ondersteunen van het neurologische symposium. Hierin heeft Bert Nelissen ook een rol gehad. Bert, dank voor het interesse in dit project en het op een kier zetten van de deur.

Peter Hilderink heeft dit project financieel en inhoudelijk gesteund vanuit de faculteit gezondheid en techniek, waarvoor dank. Ik ben blij dat ik ook kennis kon teruggeven in de vorm van studentprojecten, buitenlandse stages en hoorcolleges. Ik kijk uit naar een verdere samenwerking in de toekomst.

Paul Borm, de lector van de kenniskring centre of expertise in life sciences, was de eerste die mijn project gesteund heeft door mij een plaats te bieden in de kenniskring. Ik ben je dankbaar voor de aanhoudende ondersteuning van dit project, vooral omdat ik weet hoe ver dit thema van jou af staat.

In het begin heb ik nogal geworsteld met het vinden van 'een nest'. In mijn perceptie is een stuk creativiteit hoofdzakelijk mogelijk vanuit een veilige omgeving waarin je 'op scherp' gezet wordt. Het heeft dan ook even geduurd voordat ik de inner circle bij elkaar had. Sandra Beurskens, de lector van de kenniskring autonomie en participatie, was mijn 'starting point'. Sandra, je bent mijn maatje, degene die me in de wereld van het onderzoek heeft geïntroduceerd, de vinger op de zwakke plekken van te snel geschreven artikelen legt, die harder loopt tijdens een jog-pauze, mijn dagelijkse mentor, mijn medeontdekker van onontgonnen gebieden, mijn kamergenoot bij 
overnachtingen in Den Haag, Amsterdam, Oxford en elders op de wereld en je bent mijn sparpartner. Ik verheug me op het vervolg, vooral omdat ik weet dat jij een oogje in het zeil houdt.

Derick Wade, thank you for joining our little adventure at a very early stage of this PhD-project as my promotor. You taught me how to write a story instead of a dry-scientific-fact-paper. My first writing attempts turned out to be suicidal ones. Manuscripts corrected by you using 'track changes', were just red everywhere. However, I got my act together judging by the diminishing 'redness' of the papers that followed. I learned how to write, at least a bit. You also taught me that research and friendship can go together. Liz and you have welcomed us several times at your house and we felt at home immediately. Liz, thank you for letting us have Derick once a month for brain picking. I hope we will keep seeing each other in the future.

Jos Schols, dank dat jij ook de rol van promotor op je wilde nemen. Jij was de laatste die bij de inner circle van dit project kwam en je hebt het begeleidingsteam compleet gemaakt. Fantastisch hoe je jouw steun kunt laten voelen en hoe je binnen een uur van elke gestuurde mail reageert, onafhankelijk van waar je bent in de wereld. Dank voor je betrokkenheid bij dit project en het inbedden van dit onderzoek binnen CAPHRI van de universiteit Maastricht.

De leden van de leescommissie, Prof. Jan Hamers, Prof. André Knottnerus, Prof. Rob de Bie, Prof. Gert Kwakkel en Dr. Nikhil Sharma wil ik bedanken voor het (positief) beoordelen van de het proefconcept van het manuscript.

Een nadeel van promoveren in een organisatie die nog geen (lang) promovendiverleden of -cultuur heeft, is dat je soms met beperkte voorzieningen moet werken. Dit is echter geen enkel probleem als blijkt dat de bibliothecaris verantwoordelijk voor jouw expertisegebied Marsha Bokhorst is. Wat ben jij een kei in je vak. Dank dat je me geholpen hebt met alle literatuur die nodig was om tot goede onderbouwing te komen van onze artikelen, ethische toetsingsaanvragen, subsidieaanvragen en beleidsdocumenten.

In dit proefschrift wordt verslag gedaan van twee klinische studies, één bij patiënten na een beroerte (CVA) en één bij patiënten met de ziekte van Parkinson.

In de CVA-studie waren drie verpleeghuizen betrokken: Klevarie, Stichting Vivre in Maastricht, St. Camillus, de Zorggroep in Roermond en Sevagram, Sevagram Zorgcentra in Heerlen. Ik wil het bestuur van deze organisaties bedanken voor hun ondersteuning van dit project, vooral Mariëtte Keizer en daarnaast alle 'hoofden paramedische dienst': Gildy Hertogs, Thijs Brouns en 
Chris Slijpen. De hoofdonderzoekers op locatie, Susanne van Kroonenburgh, Jeroen Demarteau, Paul van de Laar en Paul Leurs dank ik voor de prettige samenwerking, de heldere en frequente communicatie en de aanhoudende steun. Susanne en Jeroen, in januari 2007 waren we al samen in Oxford, jullie waren erbij vanaf het prille begin. Ik heb jullie interesse en onze discussies als bijzonder positief ervaren. Dank hiervoor. In het totaal waren multidisciplinair 25 professionals betrokken bij dit deel van de studie. Ik dank jullie allen voor de kritische vragen en de betrokkenheid. Jullie hebben mij op elke locatie 'thuis' laten voelen.

Xandra Gielen en Jolanda van Haastregt hebben mij geholpen bij de procesevaluatie van de CVA studie. Hartelijk dank voor jullie hulp bij en suggesties voor het verbeteren van dit gedeelte van het onderzoek.

De studie met patiënten met de ziekte van Parkinson is uitgevoerd op vijf locaties hier in het zuiden van Nederland: fysiotherapiemaatschap Snijders te Stein, Fysiovision te Geleen, fysiotherapiepraktijk de Baandert te Sittard, Orbis medisch concern te Sittard en verpleeghuis St. Camillus te Roermond.

Ik wil graag het bestuur van de instellingen bedanken voor het vertrouwen en de medewerking aan de studies, in het bijzonder John Snijders Jr., Marc Kuijpers, Mil Cals, Max Dreesen en Thijs Brouns. De hoofdonderzoekers op locatie, Henk Pepels, Rob de Ruijter, Marcel van Meijel, Mil Cals, Paul van de Laar en Willy Keijsers bedank ik voor de prettige samenwerking!

Ik wil alle proefpersonen die deelgenomen hebben aan de verschillende studies bedanken voor de openhartigheid tijdens interviews, hun inspanningen tijdens interventies en metingen en vooral voor hun tijd die zij onbaatzuchtig in dit onderzoek gestoken hebben.

De (oud-)leden van de kenniskringen CEL en A\&P van de HsZuyd dank ik voor het sparren en meeleven. Bijzondere dank gaat uit naar Erik van Rossum, Luc de Witte en Björn Crüts en de (voormalige) AIO-soepleden Tineke Schoot, Ruth Dalemans, Jessie Lemmens en Ramon Daniels. Lotgenoten zijn iets moois. Ruth, in maart heb je even piekfijn voorgedaan hoe dat moet, promoveren: beslist doch charmant. Ik vond het geweldig.

Werken op de leukste én beste opleiding fysiotherapie van Nederland is een voorrecht. Lieve collega's, wat ben ik blij dat ik met jullie mag samenwerken. Eigenlijk hoef en wil ik hierin niet specificeren, maar wellicht is het aardig om een aantal voorbeelden te geven van hoe jullie mij geholpen hebben. Ruud Gerards en Gijs van Oorsouw, jullie hebben de deur naar Camillus geopend. 
Ruud, je bent zelfs de eerste keer daar mee naartoe gegaan. Wat heb ik dat gewaardeerd! Rob de Ruijter, Marcel van Meijel en Henk Pepels, jullie hebben mij gepord om in de eerste lijn 'een en ander uit te proberen'. Wat is dat uit de hand gelopen. Mooi! Toch?

Myriam Lamerichs, als BPG coördinator van het blok over centraal neurologische aandoeningen heb je mij vaker gevraagd een hoorcollege voor studenten te verzorgen. Dank dat de opgedane kennis terug kon vloeien in het onderwijs. Peter Konsten, dank voor het maken van de mooie Excel files voor de dataverzameling. Jos, dank voor al je waardevolle tips voor presentaties. Vera, als kamergenoten zien we elkaar wekelijks. Ik wens een ieder een kamergenoot zoals jij. Ik denk dat dan veel meer mensen het (nog) leuker zouden vinden om te gaan werken. Nancy, Huub, Loes, Dorien, Mario, Monique, Yolande, ik wil jullie bedanken voor 'je-weet-wel-wat'. Het deed me elke keer goed. Jacques, jij hebt me twee keer uitgenodigd om voor het jaarboek te schrijven en beide hoofdstukken hebben me geholpen mijn gedachten op een rijtje te krijgen, waarvoor dank. Marieke Spreeuwenberg, heb dank voor je fantastische introductie in de statistiek en je lessen waardoor het abracadabra-gehalte werd 'gereduceerd' en er (iets van) begrip voor het toveren met getallen ontstond.

Als docent aan de opleiding fysiotherapie is werken met studenten je dagelijks brood. Eén van de taken van de kenniskring is het om ook studenten, bij interesse, zo veel mogelijk bij onderzoek te betrekken. Dat heb ik geweten. Volgende studenten hebben als onderdeel van een stageopdracht of als afstudeeronderwerp 'iets' met mentale training gedaan in de periode 2005-2010 (in chronologische volgorde): Jorrit Schade van Westrum (fysio), Kenneth Oti en Rik Marcellis (BW en fysio, medebegeleider UM Jos Adam), Paul Perreijn en Thijs de Graaf (biometrie HsZuyd, medebegeleider Hub de Gussum), Danny Trines, Frank Roberts en Johan Ritzen (ICT Proeftuin HsZuyd, medebegeleider Sjef Leunissen), Axel Hagemann, Yvonne Wierts, Marek Bures, Maike Ballmann, Valerie Kiffmeyer, Dorit Teuscher, Melanie Kleynen, Maartje Schoenmakers, Susan Smeets, Laura Niessen, Jeroen Simons, Sanne Offermans, Lars Lebens, Martijn Wallink, Febe den Hollander, Daisy van Elteren, Jeroen Vonk, Yara Münnich, Theresa Kaiser, Marco VandenBoorn, Bart Oudelaar, Eva Schmitz, Simone Friedrichs, Melanie Aengenoordt, Michael Schaffarczyk, Milou Brouns, Maartje Sangen, Anna Krupp, Jenny Figge, Rebecca Riesz, Jana Mechelinck, Andrej Ruch, Dima Sterz, Nadine Landwehrs, Iris Scheepers, Ellen Hendrix, Inge Jansen, John van den Broek, Giel Winkelmolen, Leon Wulms, (fysio), Celine Huibers, Esther Molenaar (BW, medebegeleider UM Jos Adam). Machteld Schravendeel heeft bijgedragen als onafhankelijke meter. Ik wil jullie allemaal bedanken voor al jullie vragen, twijfels, soms pittige maar altijd zinvolle bijeenkomsten en gezellige evenementen (BBQ-en, Sinterklaas, Italiaanse avond). Ik wil alle medebeoodelaars van de 'afstudeermodule' bedanken voor de prettige en vooral constructieve samenwerking. 
Twee studenten wil ik met nadruk noemen. Als eerste Melanie Kleynen. Melanie, je was al fantastisch om mee te werken als student en ik was apetrots toen jij en Maartje de KNGFafstudeerprijs wonnen in 2007. Ik was blij zo'n goede kracht en integere persoon te kunnen behouden voor het project na je afstuderen, vooral omdat je waarschijnlijk elke andere job had kunnen krijgen, maar 'ja' zei tegen deze. Als onafhankelijke meter in de CVA-studie heb je veel werk verzet en de verdere, uitgebreidere samenwerking binnen jouw mastertraject epidemiologie aan de UM ervaar ik als uitdagend en stimulerend. Je bent heerlijk om mee samen te werken, koffie te drinken, te koken of gewoon mee van gedachten te wisselen. Ik hoop dat onze persoonlijke en werkrelatie voldoende tijd krijgt om verder te groeien.

Bart, op jou werd ik geattendeerd door Elma, je SLB begeleider in het eerste jaar fysiotherapie. Ze zei, dat als ik door je nonchalante houding heen kon prikken en ik je wat zou uitdagen dan zou ik een hele goede aan je hebben. Ze had gelijk. Je regelde alles perfect en je deed deze taak met veel zorgvuldigheid. Ik hoop dat je (vanuit Groningen?) in je toekomstplannen een beetje plaats gereserveerd houdt voor de HsZuyd.

Een spin off, vrij vroeg in dit project was een jaarlijks terugkerend seminar. In eerste instantie is dit idee uit pure noodzaak ontstaan. Wij hadden dringend behoefte aan een netwerk van onderzoekers die met hetzelfde thema bezig waren en bereid waren om samen te werken of informatie uit te wisselen. De eerste twee seminars gingen alleen over motor imagery en mental practice, maar vanaf het derde symposium kwam de nadruk op neurologische revalidatie algemeen te liggen. Vanuit dit seminar zijn leuke en interessante contacten ontstaan in Nederland, België, Duitsland, Zwitserland, Engeland, Italië en de VS, waarvan ik er drie hier wil noemen: Corina Schuster, Nik Sharma en Elke Heremans.

Dear Corina, we first met at the Oxford seminar on imagery and mental practice in January 2007. We were both still developing our thoughts on how to construct and perform our clinical trials. Still a bit lost. And 1,5 years later we had a room full of therapists interested in our ideas at the conference in St. Gallen. Who would have thought that would happen? I wish you the best with finishing your $\mathrm{PhD}$.

Dear Nik, what a surprise we were in when we found out you were flying in from the States to talk at this 'little seminar' in the South of Holland, for we thought you were living in the UK! I felt quite stupid not knowing this, for I suggested taking the train through the tunnel... Anyway, you came back a year later, so you must have thought it worthwhile. I hope, we can do research together in the future. 
Beste Elke, ook jij was vanaf het eerste moment bij de seminars aanwezig. Je doet zo mooi onderzoek naar mentale training en bewegingsvoorstellingen. Je lezingen zijn helder en mooi gestructureerd. Ik ben blij dat ook jij iets hebt kunnen halen bij ons jaarlijks symposium. Ik zou het begroeten als er in de toekomst meer ruimte voor uitwisseling van ideeën zou zijn. Onder leiding van Jan Engelen zijn studenten van de opleiding facility management nu al bezig met de planning en voorbereiding van het volgende symposium in oktober 2011. Ik ben ervan overtuigd dat het wederom een mooie en interessante happening gaat worden.

Nancy, Gerty en Stéphanie, heel veel dank in de ondersteuning van zo veel dingen (o.a. het symposium). Wat waardeer ik jullie creativiteit, jullie kwaliteiten als gastvrouw en acute probleemoplossers, jullie organisatietalent en werken met deadlines.

Rainier, dank voor het maken van de foto's tijdens twee symposia. Je hebt een oog voor de meest maffe details. Mooi.

De mannen van de audiovisuele dienst van de HsZuyd bedank ik voor alle ondersteuning tijdens symposia en voor het monteren en opnemen van de mooiste filmpjes voor presentaties. Bart, wat hebben wij lang op dat muziekstukje zitten klooien, maar achteraf was het meesterlijk. Jo, Bart, Hub, jullie zijn top!

Een van de mooie dingen van het langdurig werken aan een groot project is, dat je allerlei mensen leert kennen met wie je inhoudelijke raakvlakken hebt. Zo is via Thomas Schack hernieuwd contact ontstaan met de universiteit waar ik destijds 10 jaar eerder afstudeerde, de Sportuniversiteit in Keulen (DSHS).

Lieber Thomas, vielen Dank für die nette Kooperation der letzten Jahren. Auch wenn wir uns nicht so oft sehen, ich denke der Output war in Ordnung. Auch Mitarbeiter des psychologischen Instituts der DSHS in Köln und des Arbeitsbereiches Neurokognition und Bewegung - Biomechanik der Uni Bielefeld haben uns geholfen das Thema mentales Training weiter zu vertiefen und haben an verschieden neurologischen Seminars in England und Holland gesprochen. Ein herzliches Dankeschön an Frank Engel, Thomas Heinen, Heiko Lex, Christian Schütz und Matthias Weigelt für Eure Zeit und Gedanken.

Via Derick Wade is onze werkgroep in contact gekomen met Helen Dawes van Brookes University in Oxford. Dear Helen, thank you so much for bouncing thoughts, having students, coming over and replying to mails regardless of the day or night time. I am very enthusiastic 
about the Erasmus Exchange Program and look forward to continuing and fastening the connection between Heerlen and Oxford. Students and co-workers at Brookes have been great organizers supporters and speakers at the neurological seminar in the past, a special 'thank you' to Patrick Esser, Taco Bovend-eerdt and Anique Driessen. Rosa Eadle, thank you for helping us formalize the exchange program and Farida Gorab Ben, thank you for helping us with the accommodation of the HsZuyd students on campus. A special thanks goes to Karen Barker for having several of our students as interns at the Nuffield Orthopaedic Centre. They learn so much from this clinical work.

In de afgelopen periode heb ik, samen met de projectgroep, vier medisch ethische toetsingsaanvragen laten toetsen. Drie daarvan zijn getoetst door de commissie van het Atrium Medisch Centrum/Orbis medisch concern/HsZuyd. Ik wil Hélène van de Besselaar, Jacquelien Spreeuwenberg en Bert Starmans bedanken voor de prettige samenwerking en de kritische commentaren om de studies nog beter voor te bereiden.

Veel subsidie is veelal niet nodig om methodologisch zuiver onderzoek uit te voeren, maar het maakt het wel makkelijker om een beetje cash te hebben! Ik wil dan ook het Mobiliteitsfonds, ziektekostenverzekeraars VGZ en NutsOhra, ZonMW, de kenniskringen CEL en A\&P, de faculteit gezondheid en techniek en het college van bestuur bedanken voor alle financiële ondersteuning die ik de afgelopen jaren heb mogen ontvangen. Henri Holtus, jouw interesse in het project, je deelname aan voordrachten en de persoonlijke tussentijdse rapportage heb ik heel erg op prijs gesteld.

Jos Halfens en Jos Kurvers van Adelante te Hoensbroek dank ik voor het meedenken in de opstartfase van het project. Wat fijn dat jullie meegegaan zijn met mijn eerste warrige ideeën voor een onderzoeksvoorstel. Jammer dat we deze reis niet samen hebben kunnen voortzetten.

Het is best een organisatie om als moeder van twee kinderen en partner van een man met een eigen zaak de tijd te vinden om hele zinnen af te denken, laat staan te schrijven. Dit kan dan ook niet zonder de hulp van een heleboel mensen die geen officiële rol in dit project hadden. Allereerst wil ik Erica en Eelze van Goinga bedanken. Erica, zonder jou zou dit hele project nooit van de grond gekomen zijn. Jij en Eelze vangen onze kinderen op als wij aan het werk zijn. Het is voor hen een tweede thuis. Wat een geluk dat we jullie gevonden hebben. 
Alle mama's en papa's van alle vriendjes en vriendinnetjes van Moon en Teun bedank ik voor het 'opvangen', laten logeren en spelen van onze spruitjes in jullie families. Lucienne, Katinka, Maud, Mariëlle, Esther en Elke, wat fijn dat jullie me in organisatorisch moeilijke situaties uit de brand hebben geholpen. Tanja, jij bent een verhaal apart. Het is fijn om gewoon bij je langs te kunnen komen en 'thuis' te zijn. Dank daarvoor. Anita, dank voor het delen van je gedachten over het leven. Daarnaast wil ik een aantal vrienden bedanken voor de nodige ontspanning tussendoor. In willekeurige volgorde: Roger en Anita, Marcel en Ireen, Hans en Tanja, Jo en Elly, Marion en Richard, Marjon en Flip. Liebe Birgit, liebe Cordula, schön das ihr seit 20 Jahren (!) als feste, immer wieder zurückkehrende Grösse, in meinem Leben seid.

Aline, heb dank voor de mooie opmaak van dit proefschrift, vooral voor het fantastisch be- en verwerken van het schilderij en de tekeningen. Jij wist hoe belangrijk dit voor mij was.

Lieve Mom en Dad, dank dat jullie mij de afgelopen 20 jaar bij al mijn (soms onconventionele) beslissingen gesteund hebben. De manier waarop jullie het leven aangaan en jullie leven leiden is een mooi voorbeeld voor mij. Mom, ik weet dat je er ambivalente gevoelens over had, maar dank dat ik uit je mooie tekeningen en schilderijen mocht kiezen voor dit proefschrift. Maar bovenal dank voor al je wijsheid (en kledingadvies - haha). Dad, dank voor het lezen van mijn stukken en je aanhoudende interesse in het onderzoek. Jullie zouden voor mij geen betere ouders kunnen zijn. Lieve Chris en Wiebe, lieve Marc en Nicole, lieve neefjes en nichtjes, wat ben ik blij met onze grote kleine familie. Ondanks dat we elkaar niet zo vaak kunnen zien, weet ik dat als het puntje bij paaltje komt iedereen er ineens toch gewoon weer staat en dat is een heel goed gevoel. Lieve Oma visjes, Jo en Monique, dank voor het steeds weer vragen hoe het gaat.

Lieve Paul, als geen ander begreep jij dat dit project een traject was dat ik alleen moest doorlopen. Dank dat jij me daarvoor de ruimte hebt gegeven. Vijf jaar ben ik bezig geweest met patiënten die zich mooiere of betere scenario's moesten voorstellen in een lange en zware revalidatieperiode. Ik heb dat nooit hoeven doen. Mijn leven met jou en onze mooie kinderen op een oude boerderij die waarschijnlijk nooit af komt, behoeft geen vlucht uit de realiteit. Integendeel. Dank daarvoor. En zoals je vader altijd zei: 'Je weet het hè'! Lieve Moon en lieve Teun, wat ben ik blij dat jullie mijn kindjes zijn. Ik hou zo veel van jullie!

Ook voor al die anderen die meeleefden en hebben meegewerkt, maar niet zijn genoemd in dit dankwoord, dank je wel! 


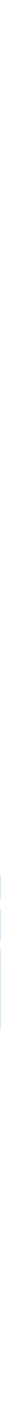

